Article

\title{
In-silico Leishmania Target Selectivity of Antiparasitic Terpenoids
}

\author{
Ifedayo Victor Ogungbe ${ }^{1, *}$ and William N. Setzer ${ }^{2}$ \\ 1 Department of Chemistry and Biochemistry, Jackson State University, Jackson, MS 39217, USA \\ 2 Department of Chemistry, University of Alabama in Huntsville, Huntsville, AL 35899, USA; \\ E-Mail: wsetzer@chemistry.uah.edu
}

* Author to whom correspondence should be addressed; E-Mail: ifedayo.v.ogungbe@jsums.edu; Tel.: +1-601-979-3719; Fax: +1-601-979-3674.

Received: 20 May 2013; in revised form: 23 June 2013 / Accepted: 26 June 2013 /

Published: 3 July 2013

\begin{abstract}
Neglected Tropical Diseases (NTDs), like leishmaniasis, are major causes of mortality in resource-limited countries. The mortality associated with these diseases is largely due to fragile healthcare systems, lack of access to medicines, and resistance by the parasites to the few available drugs. Many antiparasitic plant-derived isoprenoids have been reported, and many of them have good in vitro activity against various forms of Leishmania spp. In this work, potential Leishmania biochemical targets of antiparasitic isoprenoids were studied in silico. Antiparasitic monoterpenoids selectively docked to L. infantum nicotinamidase, L. major uridine diphosphate-glucose pyrophosphorylase and methionyl t-RNA synthetase. The two protein targets selectively targeted by germacranolide sesquiterpenoids were $L$. major methionyl t-RNA synthetase and dihydroorotate dehydrogenase. Diterpenoids generally favored docking to L. mexicana glycerol-3-phosphate dehydrogenase. Limonoids also showed some selectivity for L. mexicana glycerol-3-phosphate dehydrogenase and L. major dihydroorotate dehydrogenase while withanolides docked more selectively with L. major uridine diphosphate-glucose pyrophosphorylase. The selectivity of the different classes of antiparasitic compounds for the protein targets considered in this work can be explored in fragment- and/or structure-based drug design towards the development of leads for new antileishmanial drugs.
\end{abstract}

Keywords: antileishmanial activity; terpenoids; drug targets; docking; Leishmania 


\section{Introduction}

Several closely-related protozoan parasites in the genus Leishmania are etiological agents for a number of clinical forms of leishmaniasis. These clinical forms of leishmaniasis are characterized as either cutaneous, diffuse cutaneous, disseminated cutaneous, mucocutaneous, visceral or post-kala-azar dermal. Species causing this protozoan disease have been reported in several tropical and Neotropical regions including Africa, the Americas, Eastern Europe, central and western Asia, the Indian subcontinent as well as in Australia. There are over 350 million people at risk of infection in Leishmania-endemic regions. There are several treatment options for leishmaniasis, although the effectiveness of the available drugs depends on which clinical form is being treated, and also on the specific geographical location. For a review of the current treatment options please see reference [1]. There remains a need for better chemotherapy for cutaneous, visceral and post-kala-azar dermal leishmaniasis, as well as, Leishmania-HIV co-infection.

Current chemotherapy of visceral and cutanoeus leishmaniasis includes miltefosine, a compound that has been demonstrated to inhibit P13K/Akt signaling pathway, and fluconazole, a sterol $14 \alpha$-demethylase inhibitor. In addition to currently targeted Leishmania proteins, several other proteins have also been identified, or suggested as potential drug targets in Leishmania [2-5]. Most of these targets include enzymes that are critical to the metabolism of glucose, sterols, nucleotides and glycosylphosphatidylinositol, as well as enzymes important for the maintenance of trypanothione and polyamine levels. Many of these proteins have been shown to be important to the survival of the parasites. Other targets include cyclin-dependent- and mitogen-activated protein kinases, topoisomerases and cathepsin-like proteases.

Some of the enzymes that are involved in glucose metabolism and are potential drug targets in some species of Leishmania include pyruvate kinase (PYK) [6,7], phosphoglucose isomerase (PGI) [8,9], uridine diphosphate-glucose pyrophosphorylase (UGPase) [10], glyceraldehyde-3-phosphate dehydrogenase (GAPDH) [11-13], glycerol-3-phosphate dehydrogenase (GPDH) [14,15], triosephosphate isomerase (TIM) [16-18], thiol-dependent reductase I (TDR1) [19] and phosphomannomutase (PMM). TDR1 is involved in the regulation of the terminal steps of glycolysis and the enzyme fortuitously catalyzes the activation of antiparasitic antimonial prodrugs while PMM catalyzes the conversion of mannose-6phosphate to mannose-1-phosphate, which is essential for the biosynthesis of glycoconjugates, and it has been suggested to be a potential drug target [20]. The enzyme that catalyzes the trypanothionecoupled conversion of methylglyoxal, a toxic byproduct of glycolysis, to lactate in Leishmania, glyoxalase II (GLO2), has also been suggested as a drug target [21,22] although modeling studies of the enzyme have suggested that inhibition of glyoxalase II will have little effect on toxic glyoxal build up in the cell [23]. In addition to these, the cysteine protease, cathepsin B (CatB) [24,25], as well as oligopeptidase B (OPB) [26-28] are also being investigated as potential drug targets. A number of proteins involved in nucleoside and nucleotide metabolism in Leishmania have also been investigated as druggable targets. These includes dihydroorotate dehydrogenase (DHODH), an enzyme involved in the de novo synthesis of pyrimidine [29,30], deoxyuridine triphosphate nucleotidohydrolase (dUTPase), an enzyme involved in controlling intracellular dUTP levels [31-33], nicotinamidase (PnC1), an essential enzyme for the production of $\mathrm{NAD}^{+}$[34], nucleoside hydrolase (NH) [35-37], nuceloside diphosphate kinase $\mathrm{b}$ (NDKb) [38] as well as phosphodiesterase 1 (PDE1) [39-41] and 
pteridine reductase 1 (PTR1) [42-45]. Also, proteins involved in co-/post-translational protein processing like $N$-myristoyltransferase (NMT) [46-48] and cyclophilins (Cyp) [49,50] have being actively investigated as antileishmanial drug targets as well as charged-tRNA synthesizing enzymes, methionyltRNA synthetase [51] and tyrosyl-tRNA synthetase [52].

Numerous phytochemical agents have exhibited either in vitro or in vivo antileishmanial activity [5,53-61]. While the activities of many of these compounds are notable, what is generally unknown are the biochemical targets of these agents. In this study, we have carried out a molecular docking analysis of known antiparasitic plant-derived isoprenoids with established drug targets with known structures available from the Protein Data Bank.

Figure 1. Monoterpenoids examined in this study.

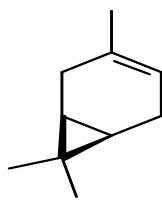

$\delta$-3-Carene<smiles>Cc1ccc(C(C)C)cc1</smiles><smiles>[Ca][Ca][Te]</smiles><smiles>C=C(C)C1CC=C(C)CC1</smiles>

$(S)$-Limonene<smiles>C=C(C)C1CC=C(CO)CC1</smiles>

Perilla alcohol<smiles>CC1=CC(=O)[C@H](C(C)C)CC1</smiles>

(R)-Piperitone<smiles>CC1=CCC(=C(C)C)CC1</smiles>

Terpinolene<smiles>CC12CCC(C)(C(=O)C1)C2(C)C</smiles>

Camphor<smiles>CC(C)=CCCC(C)=CC=O</smiles>

Geranial<smiles>C=C(C)C1CCC2(C)OC2C1</smiles>

Limonene oxide<smiles>CC1=CCC(C(C)C)C=C1</smiles>

(R)- $\alpha$-Phellandrene<smiles>CC1=CC(=O)C(C(C)C)CC1</smiles>

(S)-Piperitone

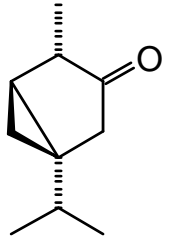

$\alpha$-Thujone<smiles>Cc1ccc(C(C)C)cc1O</smiles>

Carvacrol<smiles>CC(C)=CCCC(C)=CCO</smiles>

Geraniol<smiles>C=CC(C)(O)CCC=C(C)C</smiles>

Linalool<smiles>CC1=CC[C+](C(C)C)C=C1</smiles>

(S)- $\alpha$-Phellandrene<smiles>C=C1CCC2(C(C)C)CC1C2</smiles>

Sabinene<smiles>CC(C)[C@]12CC(=O)[C@H](C)[C@H]1C2</smiles>

$\beta$-Thujone<smiles>C=C(C)[C@H]1CC=C(C)C(=O)C1</smiles>

(S)-Carvone<smiles>C=C(C)C1CC[C@@H](C)CC1O</smiles>

Isopulegol<smiles>CC1(C)C2CC=C(C=O)C1C2</smiles>

Myrtenal

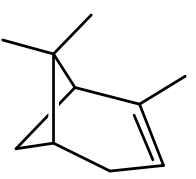

$\alpha$-Pinene<smiles>CC1=CCC(C(C)C)=CC1</smiles>

$\gamma$-Terpinene<smiles>Cc1ccc(C(C)C)c(O)c1</smiles>

Thymol<smiles>CC12CCC(C(C)(C)C)(CC1)O2</smiles>

1,8-Cineole<smiles>C=C(C)[C]1CC=C(C)CC1</smiles>

(R)-Limonene<smiles>CC(C)=CCC/C(C)=C\C=O</smiles>

Neral

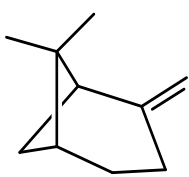

$\beta$-Pinene<smiles>CC1=CCC(O)(C(C)C)CC1</smiles>

Terpinen-4-ol<smiles>CC1=CC(=O)C2CC1C2(C)C</smiles>

Verbenone 
Table 1. MolDock docking energies (kJ/mol) of monoterpenoids with Leishmania major protein targets.

\begin{tabular}{|c|c|c|c|c|c|c|c|c|c|c|c|c|}
\hline Monoterpenoids & LmajCatB & LmajDHODH & LmajdUTPase & LmajNDKb & LmajNH & LmajNMT & LmajOPB & LmajPDE1 & LmajPTR1 & LmajMetRS & LmajTyrRS & LmajUGPase \\
\hline$\delta$-3-Carene & -43.8 & -63.0 & -50.0 & -56.3 & -51.0 & -52.9 & -62.7 & -56.9 & -54.9 & -60.8 & -63.2 & -65.1 \\
\hline Camphor & -43.2 & -61.3 & -39.2 & -52.4 & -50.4 & -50.0 & -49.1 & -58.1 & -43.6 & -52.0 & -44.6 & -60.9 \\
\hline Carvacrol & -46.5 & -64.8 & -58.9 & -61.9 & -52.7 & -69.9 & -66.6 & -62.6 & -62.1 & -70.9 & -66.7 & -66.2 \\
\hline (S)-Carvone & -46.7 & -68.9 & -56.0 & -63.6 & -53.2 & -55.8 & -56.0 & -63.6 & -56.6 & -69.2 & -61.5 & -66.5 \\
\hline 1,8-Cineole & -37.1 & -57.6 & -37.8 & -50.6 & -43.5 & -45.7 & -42.5 & -53.6 & -39.7 & -46.8 & -45.4 & -59.6 \\
\hline$p$-Cymene & -40.8 & -55.7 & -54.6 & -54.8 & -51.4 & -63.0 & -57.6 & -58.1 & -56.5 & -61.9 & -63.6 & -60.7 \\
\hline Geranial & -52.8 & -69.0 & -67.3 & -77.1 & -65.8 & -75.6 & -63.8 & -66.7 & -72.8 & -76.8 & -73.7 & -76.9 \\
\hline Geraniol & -55.9 & -69.7 & -68.9 & -73.5 & -67.5 & -72.8 & -68.0 & -65.1 & -70.2 & -76.5 & -72.1 & -74.1 \\
\hline Isopulegol & -46.3 & -64.8 & -51.1 & -59.0 & -55.4 & -63.6 & -54.7 & -57.0 & -53.8 & -61.0 & -65.0 & -62.6 \\
\hline$(R)$-Limonene & -46.1 & -61.6 & -49.6 & -59.5 & -47.5 & -52.4 & -56.4 & -57.6 & -57.2 & -63.4 & -58.4 & -65.6 \\
\hline$(S)$-Limonene & -44.3 & -58.7 & -56.4 & -56.2 & -50.0 & -65.9 & -59.5 & -58.6 & -56.3 & -64.3 & -66.0 & -62.0 \\
\hline Limonene oxide & -44.1 & -65.2 & -48.2 & -57.7 & -52.5 & -52.5 & -59.6 & -59.0 & -50.1 & -59.0 & -52.0 & -64.8 \\
\hline Linalool & -52.3 & -67.4 & -63.9 & -70.1 & -62.0 & -71.0 & -63.2 & -65.5 & -65.7 & -73.7 & -72.5 & -71.9 \\
\hline Myrtenal & -47.1 & -64.3 & -45.7 & -56.5 & -48.8 & -51.5 & -60.8 & -61.8 & -51.7 & -57.3 & -55.7 & -66.2 \\
\hline Neral & -49.6 & -72.2 & -68.3 & -76.6 & -68.0 & -75.2 & -64.6 & -67.3 & -71.8 & -75.3 & -71.0 & -75.6 \\
\hline Perilla alcohol & -49.0 & -63.1 & -58.2 & -60.9 & -52.3 & -72.9 & -68.1 & -64.0 & -63.5 & -73.6 & -72.9 & -63.5 \\
\hline$(R)$ - $\alpha$-Phellandrene & -41.7 & -61.6 & -53.2 & -59.8 & -51.0 & -53.8 & -56.4 & -55.9 & -59.2 & -66.7 & -60.8 & -66.4 \\
\hline$(S)$ - $\alpha$-Phellandrene & -42.2 & -60.3 & -54.5 & -57.8 & -51.9 & -65.1 & -58.6 & -58.3 & -55.0 & -65.3 & -64.7 & -63.6 \\
\hline$\alpha$-Pinene & -40.0 & -54.9 & -39.3 & -52.0 & -45.1 & -44.9 & -53.4 & -53.4 & -42.0 & -53.4 & -49.4 & -60.6 \\
\hline$\beta$-Pinene & -39.6 & -55.5 & -39.8 & -52.6 & -47.0 & -45.3 & -52.9 & -53.7 & -41.3 & -54.3 & -47.5 & -60.4 \\
\hline$(R)$-Piperitone & -45.9 & -63.0 & -54.9 & -60.2 & -51.9 & -67.0 & -60.2 & -63.1 & -62.0 & -63.8 & -65.0 & -67.9 \\
\hline$(S)$-Piperitone & -46.8 & -67.4 & -54.6 & -61.2 & -50.8 & -60.8 & -61.2 & -62.3 & -59.7 & -65.6 & -64.9 & -68.0 \\
\hline Sabinene & -43.7 & -63.4 & -54.6 & -63.3 & -51.4 & -55.8 & -58.9 & -57.5 & -58.4 & -66.5 & -62.0 & -67.0 \\
\hline$\gamma$-Terpinene & -41.1 & -57.4 & -56.0 & -56.0 & -52.4 & -65.5 & -58.8 & -59.7 & -57.5 & -63.5 & -65.5 & -62.0 \\
\hline Terpinen-4-ol & -47.5 & -62.5 & -57.4 & -59.7 & -53.2 & -60.8 & -58.9 & -61.0 & -61.7 & -66.9 & -69.8 & -64.4 \\
\hline Terpinolene & -46.2 & -59.8 & -52.6 & -55.3 & -49.1 & -58.8 & -55.3 & -58.5 & -57.0 & -62.8 & -62.3 & -61.2 \\
\hline$\alpha$-Thujone & -48.4 & -73.0 & -55.8 & -62.2 & -51.7 & -59.0 & -58.6 & -64.3 & -63.5 & -73.8 & -62.1 & -67.5 \\
\hline$\beta$-Thujone & -47.6 & -69.7 & -58.2 & -60.2 & -56.9 & -59.7 & -62.8 & -65.0 & -62.3 & -71.0 & -62.5 & -67.9 \\
\hline Thymol & -45.8 & -61.7 & -55.7 & -60.9 & -52.3 & -68.7 & -59.2 & -64.2 & -62.9 & -65.6 & -69.5 & -63.8 \\
\hline Verbenone & -41.4 & -62.0 & -44.3 & -57.5 & -49.4 & -51.0 & -48.7 & -58.2 & -47.0 & -53.0 & -52.7 & -63.2 \\
\hline
\end{tabular}


Table 2. MolDock docking energies (kJ/mol) of monoterpenoids with Leishmania donovani and L. mexicana protein targets.

\begin{tabular}{|c|c|c|c|c|c|c|c|c|c|c|c|c|}
\hline Monoterpenoids & LdonCatB & LdonCyp & LdonDHODH & LdonNMT & LmexGAPDH & LmexGPDH & LmexPGI & LmexPMM & $\begin{array}{c}\text { LmexPYK } \\
\text { Site } 1 \\
\end{array}$ & $\begin{array}{c}\text { LmexPYK } \\
\text { Site } 2 \\
\end{array}$ & $\begin{array}{c}\text { LmexPYK } \\
\text { Site } 3 \\
\end{array}$ & LmexTIM \\
\hline$\delta$-3-Carene & -50.9 & -56.1 & -59.4 & -49.5 & -53.7 & -59.1 & -43.5 & -51.8 & -58.9 & -50.8 & -59.4 & -58.0 \\
\hline Camphor & -42.7 & -48.0 & -62.2 & -47.6 & -44.4 & -58.8 & -40.9 & -50.4 & -50.2 & -45.7 & -55.1 & -45.9 \\
\hline Carvacrol & -58.3 & -63.8 & -57.9 & -64.9 & -56.0 & -61.2 & -48.6 & -57.9 & -63.9 & -53.4 & -65.5 & -56.3 \\
\hline (S)-Carvone & -54.0 & -65.6 & -60.3 & -59.7 & -49.6 & -61.2 & -49.5 & -58.3 & -62.4 & -55.5 & -62.9 & -54.1 \\
\hline 1,8-Cineole & -36.3 & -46.8 & -53.6 & -38.0 & -39.8 & -51.1 & -37.6 & -47.1 & -52.5 & -42.7 & -50.3 & -48.3 \\
\hline$p$-Cymene & -51.7 & -54.5 & -51.4 & -54.7 & -48.4 & -56.1 & -50.2 & -52.9 & -58.0 & -53.4 & -60.4 & -55.2 \\
\hline Geranial & -68.7 & -67.2 & -65.2 & -75.0 & -66.6 & -70.4 & -74.4 & -63.9 & -71.1 & -61.2 & -75.2 & -71.4 \\
\hline Geraniol & -69.3 & -68.2 & -65.8 & -71.7 & -64.2 & -72.4 & -70.1 & -63.1 & -71.3 & -61.6 & -75.5 & -70.1 \\
\hline Isopulegol & -52.9 & -61.1 & -57.6 & -65.2 & -57.9 & -60.9 & -44.9 & -57.6 & -63.1 & -55.3 & -59.3 & -59.4 \\
\hline$(R)$-Limonene & -52.1 & -58.6 & -57.2 & -51.8 & -49.5 & -58.1 & -43.4 & -53.3 & -56.9 & -51.1 & -58.1 & -53.1 \\
\hline$(S)$-Limonene & -54.5 & -57.2 & -50.5 & -62.3 & -51.9 & -58.7 & -43.5 & -55.3 & -61.3 & -54.8 & -62.2 & -56.9 \\
\hline Limonene oxide & -46.2 & -58.4 & -63.9 & -33.4 & -54.1 & -61.1 & -44.3 & -53.9 & -58.3 & -52.2 & -61.2 & -57.6 \\
\hline Linalool & -65.1 & -65.1 & -70.5 & -65.6 & -61.4 & -67.8 & -58.6 & -66.9 & -68.7 & -60.1 & -74.8 & -65.9 \\
\hline Myrtenal & -47.7 & -59.2 & -60.1 & -36.7 & -49.1 & -60.2 & -45.0 & -52.6 & -57.7 & -50.4 & -55.8 & -53.6 \\
\hline Neral & -64.4 & -69.2 & -70.5 & -71.7 & -66.1 & -72.9 & -58.7 & -63.2 & -71.8 & -65.2 & -74.2 & -65.5 \\
\hline Perilla alcohol & -58.7 & -65.2 & -57.5 & -71.0 & -60.3 & -63.1 & -50.4 & -57.7 & -67.0 & -57.8 & -68.7 & -60.7 \\
\hline$(R)$ - $\alpha$-Phellandrene & -52.8 & -58.6 & -58.9 & -54.9 & -53.6 & -57.0 & -52.9 & -51.0 & -60.9 & -50.9 & -59.7 & -55.8 \\
\hline$(S)$ - $\alpha$-Phellandrene & -53.4 & -59.1 & -55.0 & -59.2 & -52.5 & -57.2 & -47.7 & -53.3 & -61.9 & -52.7 & -62.1 & -55.2 \\
\hline$\alpha$-Pinene & -40.5 & -52.5 & -56.2 & -37.5 & -43.7 & -53.0 & -39.9 & -47.1 & -50.7 & -42.8 & -49.6 & -46.4 \\
\hline$\beta$-Pinene & -39.7 & -53.2 & -57.4 & -39.5 & -45.2 & -52.4 & -39.2 & -47.3 & -49.9 & -41.8 & -50.7 & -48.2 \\
\hline (R)-Piperitone & -57.5 & -66.1 & -54.1 & -65.1 & -57.1 & -60.2 & -50.4 & -58.0 & -62.7 & -54.8 & -61.9 & -58.4 \\
\hline$(S)$-Piperitone & -55.3 & -63.2 & -64.2 & -59.3 & -53.8 & -61.2 & -46.7 & -57.4 & -63.8 & -56.1 & -62.2 & -56.4 \\
\hline Sabinene & -49.3 & -59.0 & -57.9 & -59.4 & -50.9 & -59.9 & -46.0 & -52.1 & -61.0 & -51.6 & -61.2 & -55.8 \\
\hline$\gamma$-Terpinene & -53.1 & -56.2 & -54.0 & -59.5 & -50.6 & -57.9 & -49.2 & -54.0 & -60.4 & -55.7 & -62.2 & -56.8 \\
\hline Terpinen-4-ol & -52.4 & -63.8 & -55.1 & -64.6 & -51.4 & -61.7 & -51.8 & -56.9 & -64.5 & -56.9 & -65.6 & -58.7 \\
\hline Terpinolene & -54.1 & -57.8 & -52.6 & -57.7 & -48.0 & -57.2 & -45.3 & -51.9 & -58.9 & -51.3 & -58.9 & -54.7 \\
\hline$\alpha$-Thujone & -51.3 & -63.8 & -63.6 & -53.2 & -56.1 & -65.0 & -49.8 & -56.6 & -65.2 & -54.2 & -62.2 & -57.7 \\
\hline$\beta$-Thujone & -56.3 & -61.6 & -60.7 & -58.1 & -53.2 & -65.6 & -48.2 & -58.0 & -65.1 & -54.7 & -61.0 & -59.9 \\
\hline Thymol & -55.8 & -61.3 & -54.5 & -64.1 & -52.1 & -62.3 & -47.2 & -56.6 & -65.2 & -56.3 & -61.7 & -57.1 \\
\hline Verbenone & -40.9 & -58.0 & -61.6 & -45.4 & -48.1 & -59.6 & -42.4 & -52.2 & -56.9 & -49.2 & -58.0 & -48.9 \\
\hline
\end{tabular}


Table 3. MolDock docking energies $(\mathrm{kJ} / \mathrm{mol})$ of monoterpenoids with Leishmania infantum protein targets.

\begin{tabular}{|c|c|c|c|c|c|}
\hline Monoterpenoids & LinfCYP51 & LinfGLO2 & LinfPnC1 & LinfTDR1 & LinfTR \\
\hline$\delta$-3-Carene & -50.4 & -48.0 & -66.1 & -45.9 & -56.3 \\
\hline Camphor & -46.1 & -37.8 & -56.8 & -43.8 & -52.1 \\
\hline Carvacrol & -59.5 & -53.8 & -71.0 & -53.5 & -60.9 \\
\hline (S)-Carvone & -54.9 & -47.9 & -73.6 & -52.1 & -57.6 \\
\hline 1,8-Cineole & -43.3 & -34.9 & -54.4 & -39.5 & -50.6 \\
\hline$p$-Cymene & -53.2 & -52.1 & -64.1 & -49.6 & -57.8 \\
\hline Geranial & -63.0 & -61.7 & -75.0 & -64.3 & -70.1 \\
\hline Geraniol & -64.5 & -59.1 & -74.8 & -64.2 & -69.1 \\
\hline Isopulegol & -50.8 & -49.6 & -67.6 & -49.8 & -58.6 \\
\hline$(R)$-Limonene & -51.6 & -51.4 & -68.8 & -48.4 & -54.0 \\
\hline$(S)$-Limonene & -53.0 & -52.7 & -65.2 & -49.1 & -60.0 \\
\hline Limonene oxide & -51.6 & -46.6 & -64.8 & -45.4 & -54.0 \\
\hline Linalool & -61.6 & -56.1 & -72.7 & -62.2 & -66.1 \\
\hline Myrtenal & -48.7 & -45.2 & -63.2 & -47.6 & -54.1 \\
\hline Neral & -60.7 & -57.1 & -76.4 & -61.4 & -68.1 \\
\hline Perilla alcohol & -61.9 & -60.0 & -69.3 & -57.8 & -65.5 \\
\hline$(R)$ - $\alpha$-Phellandrene & -51.1 & -50.9 & -68.1 & -48.4 & -56.1 \\
\hline$(S)-\alpha$-Phellandrene & -53.9 & -51.2 & -66.3 & -50.6 & -59.4 \\
\hline$\alpha$-Pinene & -42.4 & -38.4 & -56.9 & -41.0 & -47.3 \\
\hline$\beta$-Pinene & -43.0 & -37.5 & -56.9 & -41.6 & -48.3 \\
\hline$(R)$-Piperitone & -54.9 & -50.2 & -70.9 & -51.3 & -62.8 \\
\hline (S)-Piperitone & -54.2 & -51.6 & -73.0 & -54.2 & -60.3 \\
\hline Sabinene & -52.9 & -53.8 & -68.2 & -54.4 & -58.8 \\
\hline$\gamma$-Terpinene & -54.8 & -53.0 & -65.8 & -49.7 & -59.2 \\
\hline Terpinen-4-ol & -53.2 & -51.3 & -69.4 & -50.4 & -63.3 \\
\hline Terpinolene & -52.4 & -51.3 & -67.6 & -49.6 & -59.2 \\
\hline$\alpha$-Thujone & -53.8 & -53.9 & -74.5 & -54.0 & -60.4 \\
\hline$\beta$-Thujone & -54.5 & -50.8 & -69.4 & -52.0 & -64.7 \\
\hline Thymol & -60.2 & -53.5 & -71.5 & -52.9 & -59.6 \\
\hline Verbenone & -50.2 & -42.2 & -63.0 & -48.0 & -50.5 \\
\hline
\end{tabular}

\section{Results and Discussion}

\subsection{Monoterpenoid Docking}

The structures of the monoterpenoids examined in this study are shown in Figure 1, while the corresponding docking energies are summarized in Tables 1-3. The overall strongest docking monoterpenoid ligands were the acyclic geranial, geraniol, and neral, probably owing to their flexibility. These ligands, however, did not show docking selectivity to any of the Leishmania protein targets, but rather docked strongly to most of the proteins investigated. The protein targets that showed predominantly strong docking by monoterpenoids were L. major uridine diphosphate-glucose pyrophosphorylase (LmajUGPase), L. major methionyl t-RNA synthetase (LmajMetRS), and L. infantum nicotinamidase (LinfPnC1). Geranial had a docking energy of $-76.9 \mathrm{~kJ} / \mathrm{mol}$ with 
LmajUGPase, comparable in docking energy with several other proteins. Both enantiomers of piperitone showed significantly stronger docking to Lmaj UGPase $(-68.0 \mathrm{~kJ} / \mathrm{mol})$ than the other targets, suggesting selectivity for that protein. Geranial was also the strongest docking ligand with LmajMetRS $(-76.8 \mathrm{~kJ} / \mathrm{mol})$, but perilla alcohol $(-73.6 \mathrm{~kJ} / \mathrm{mol})$ was selective for that protein target. Carvone, piperitone, and $\alpha$-thujone showed significantly selective docking to LinfPnC1 (docking energies less than $-73 \mathrm{~kJ} / \mathrm{mol}$ ). Interestingly, although the monoterpenoids showed a docking propensity for LinfPnC1, higher terpenoids (sesquiterpenoids, diterpenoids, and triterpenoids) showed very little inclination to dock to this protein, generally with positive docking energies (see below).

Monoterpenoids represents a very small percentage of terpene-derived compounds that have been reported to have antileishmanial activity, and the docking energies of monoterpenoids were generally weaker than those obtained for limonoids, withanolides, triterpenoids, steroids and diterpenoids with these same targets (see below). Their docking energies were much higher than the energies obtained for the co-crystallized ligands of those protein targets. The higher docking energies of these compounds correlate with their small size (and molecular weight), and the minimal intermolecular interactions they are able to have with the protein targets. So, comparatively, it appears that monoterpenoids will not be prime leads for structure-based antileishmanial drug discovery. However, they may be useful in fragment-based drug discovery [62,63]. Additionally, several terpene-derived compounds are used in topical formulations. Therefore, those monoterpenoids that have antileishmanial activity and no reported toxicity at physiologically relevant concentration/dosage should be evaluated as possible components of topical polytherapy for leishmaniasis.

\subsection{Sesquiterpenoid Docking}

Sesquiterpenoids examined in this work are shown in Figures 2-5; docking energies of sesquiterpenoids are summarized in Tables 4-15. The germacranolide sesquiterpenoids exhibited the overall strongest docking energies toward the Leishmania protein targets, with 16,17-dihydrobrachycalyxolide the strongest-docking germacranolide. This ligand showed docking selectivity toward LmajMetRS (docking energy $=-152.9 \mathrm{~kJ} / \mathrm{mol}$ ) and L. mexicana phosphor-mannomutase (LmexPMM) (docking energy $=-136.3 \mathrm{~kJ} / \mathrm{mol}$ ). The two proteins most selectively targeted by the germacranolides in terms of docking energies were LmajMetRS and L. major dihydroorotate dehydrogenase (LmajDHODH). Although most germacranolides did not dock with LinfPnC1, tatridin A did show docking selectivity for this protein target. Based on molecular weight, the strongest-docking germacranolide was $4 \alpha, 5 \beta$-epoxy- 8 -epi-inunolide, and this ligand showed docking selectivity toward both LmajMet RS and LmajDHODH. 
Figure 2. Germacranolide sesquiterpenoids examined in this work.<smiles>CCOCC1=C2OC(=O)[C@H](OCC)C/C2=C/[C@@]2(C)CCC(O)(O2)C1(C)O</smiles>

8-Acetyl-13-O-

ethylpiptocarphol<smiles>CC(=O)OCC1=C2C(=C[C@@]3(C)CCC(O)(O3)C2(O)O)C[C@@H](OC(C)=O)C1=O</smiles>

8,13-Diacetylpiptocarphol

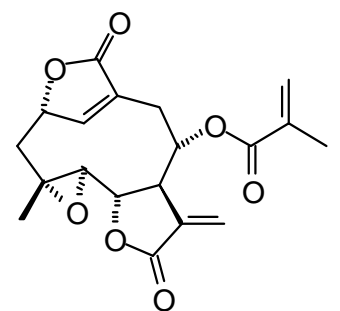

Elephantopin<smiles>C=C(C)C1(O)OC2C[C@@]3(C)OC(=CC(C)=C[C@@H]3OC2=O)C1(C)O</smiles>

Eremantholide C<smiles></smiles><smiles>[131Os]</smiles>

Hydroxymethacryloxyhirsutinolide 13-acetate

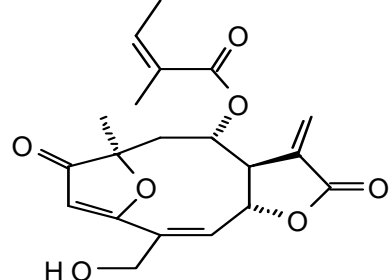

Centratherin

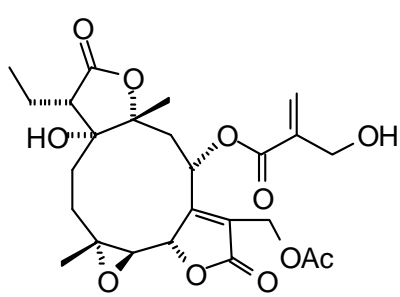

$16 \alpha, 17-$

Dihydrobrachycalyxolide<smiles>C[C@H]1C(=O)O[C@H]2C[C@@]34OC3CC[C@@]3(C)O[C@H]3C[C@H]2[C@H]14</smiles>

12,11-epi-Ivaxillin

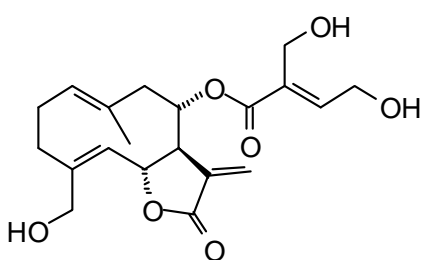

Eupatoriopicrin<smiles>C=C1C(=O)O[C@H]2C1CC(=O)C(C)CCC[C@](C)(O)C2OC(=O)C(C)CC</smiles>

Ineupatorolide A<smiles>C=C1C(=O)O[C@@H]2/C=C(\C)CCC=C1CC2</smiles>

Costunolide<smiles>C=C1C(=O)O[C@H]2C[C@]3(C[C@H]12)C[C@@]12OC1CC[C@@]2(C)O3</smiles>

11(13)-Dehydroivaxillin<smiles>C=C1C(=O)O[C@@H]2C/C(C)=C/CCC(C)(C)[C@H](O)[C@H]1[C@H]2C(=O)O</smiles>

4,5 $\alpha$-Epoxy-6 $\alpha$-acetoxy-

1(10)E,11(13)-

germacradien-12,8 $\alpha$-olide<smiles>C=C(C)C(=O)OC1CC2(C)OC(=CC3=CC(=O)C(C)(C3)O2)C=C2OC(=O)C(=C)C21</smiles>

Goyazensolide

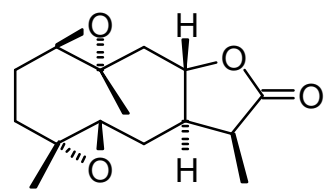

Ivaxillin<smiles>C=C(C)C(=O)O[C@H]1C/C(C)=C/C2(OC)C=C(C)[C@@H](O2)C2C(=C)C(=C)C(=O)OC21</smiles>

2-Deethoxy-2 $\beta$ methoxyphantomolin

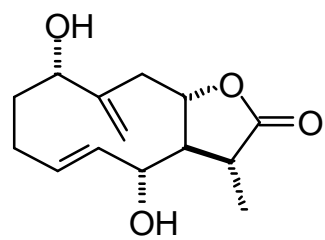

11ß,13-Dihydrotanachin<smiles>C=C1C(=O)O[C@@H]2C/C(C)=C/CC[C@]3(C)O[C@H]3CC12</smiles>

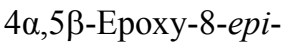
inunolide<smiles>C=C1C(=O)O[C@H]2/C=C(\C)C(O)CC=C1CC2</smiles>

Hanphyllin

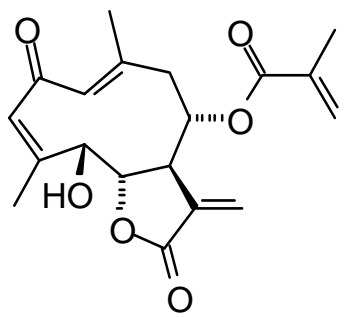

Molephantin 
Figure 2. Cont.<smiles>C=C1C(=O)O[C@H]2CC(C)/C=C\C(=O)[C@](C)(O)[C@H](OC(C)=O)C1C2OC(=O)CC(C)C</smiles>

Neurolenin B<smiles>C=C1C(=O)O[C@@H]2/C=C/C(=O)/C=C/[C@@](C)(O)CC(OC(=O)C(C)C)C12</smiles>

Tagitinin $\mathrm{C}$<smiles>CC(=O)OCC1=C2/C(=C\[C@]3(C)CC[C@@](O)(O3)[C@@H](C)C1)OC(=O)C2COC(=O)/C(C)=C\CO</smiles>

Vernolide D<smiles>C=C1C[C@@H](CC(C)/C=C\C(=O)[C@](C)(O)[C@H](OC(=O)CC(C)C)C(O)C2C(=C)C(=O)C(=O)[C@@H]2O)OC1=O</smiles>

Neurolenin C<smiles>C=C1C[C@@H]2OC(=O)C(=C)[C@H]2/C=C(/C)CC[C@H]1O</smiles>

Tanachin<smiles>C=C1C(=O)O[C@@H]2CC(C)/C=C\C(=O)[C@](C)(O)[C@@H](O)C(OC(=O)CC(C)C)CC12</smiles>

Neurolenin D<smiles>C=C1C(=O)O[C@H]2/C=C(\C)[C@H](O)CC/C(C)=C\[C@H](O)[C@H]12</smiles>

Tatridin A<smiles>C=C1C(=O)O[C@H]([C@H]2CC[C@@]34O[C@@]3(C)CCC=C4C2)[C@@H]1C</smiles>

Parthenolide<smiles>C/C=C(\C)C(=O)O[C@H]1C[C@@H](C)[C@]2(O)CC[C@](C)(/C=C3/OC(=O)C(COC(C)=O)=C31)O2</smiles>

8-Tigloylhirsutinolide 13-acetate

Figure 3. Guaianolide sesquiterpenoids examined in this work.

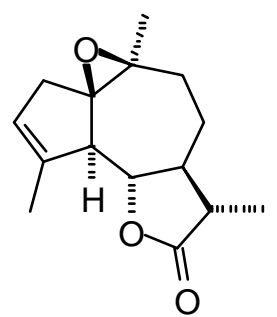

Arborescin

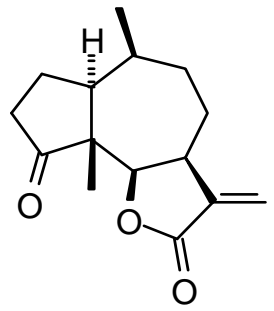

Damsin<smiles>C=C1O[C@H]2C[C@H](C)[C@H]3CCC(=O)[C@@]3(C)[C@H](O)C2C1=C</smiles>

Carpesiolin<smiles>C=C1C(=O)O[C@H]2C1CCC(=C)[C@H]1CC[C@](C)(O)[C@@H]12</smiles>

11,13-Dehydrocompressanolide

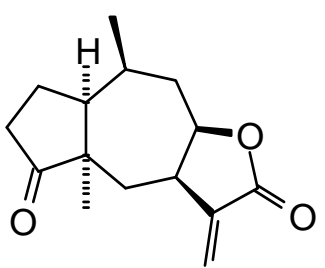

Confertin

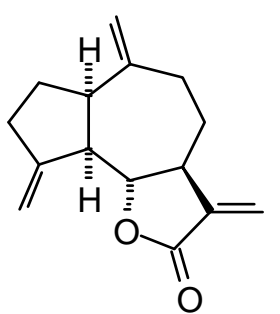

Dehydrocostus-lactone<smiles>C=C(CO)C(=O)O[C@H]1CC(=C)[C@H]2CC(O)C(=C)[C@@H]2[C@H]2OC(=O)C(=C)[C@H]12</smiles>

Cynaropicrin<smiles>C=C1C(=O)O[C@H]2[C@@H]1CCC(C)=C1C(=O)C=C(C)[C@H]12</smiles>

Dehydroleucodine 
Figure 3. Cont.<smiles>C=C1C(=O)O[C@H]2C1CCC(=C)[C@H]1CC(=O)C(=C)[C@@H]12</smiles>

Dehydrozaluzanin C<smiles>C=C1C(=O)O[C@@H]2C[C@H](C)[C@H]3C=CC(=O)[C@@]3(C)[C@H](O)[C@@H]12</smiles>

Helenalin
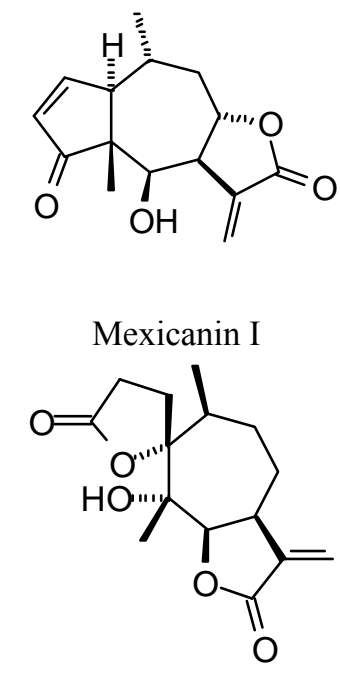

Psilostachyin

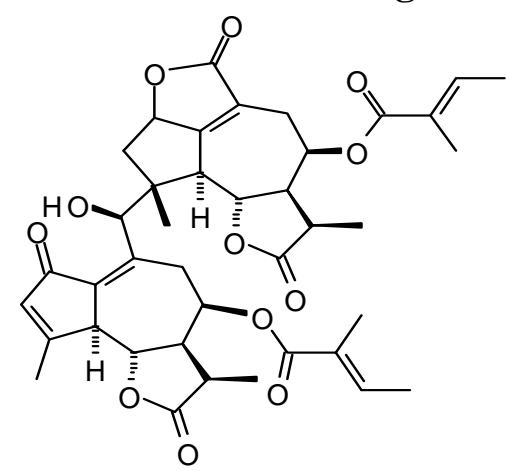

Diguaiaperfolin<smiles>C=C1C(=O)O[C@@H]2C[C@H](C)[C@H]3C=CC(=O)[C@]3(C)[C@H](OC(C)=O)[C@@H]12</smiles>

Helenalin acetate<smiles>C=C/C=C\C(=C)CC(=O)O[C@H]1CC(C)=C2C(=O)C=C(CO)[C@H]2[C@H]2OC(=O)C(=C)[C@H]12</smiles>

Lactucopicrin<smiles>C=C1C(=O)O[C@@H]2[C@@H]3CCC(=O)O[C@]3(C)[C@@H](C)CC[C@H]12</smiles>

Psilostachyin C

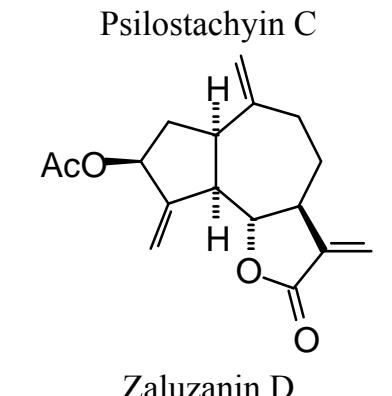

Zaluzanin D<smiles>C=C1C(=O)O[C@@H]2C[C@H](C)C3=CC(=O)OC3C[C@H]12</smiles>

4,15-Dinor-1,11(13)xanthadiene-3,5 $\beta: 12,8 \beta$ diolide<smiles>C=C1CC(O)[C@H]2C(=C)C(=O)O[C@@H]2[C@H]2C(=C)C(OC(C)=O)C[C@H]12</smiles>

$8 \beta$-Hydroxyzaluzanin D

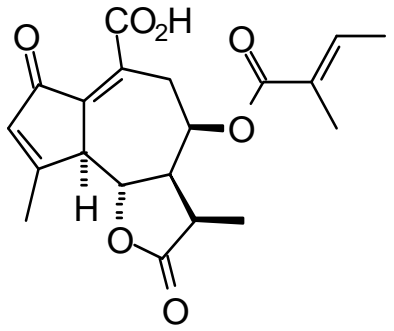

2-Oxo-8-tigloyloxyguaia1(10),3-diene-6,12-olide-14carboxylic acid
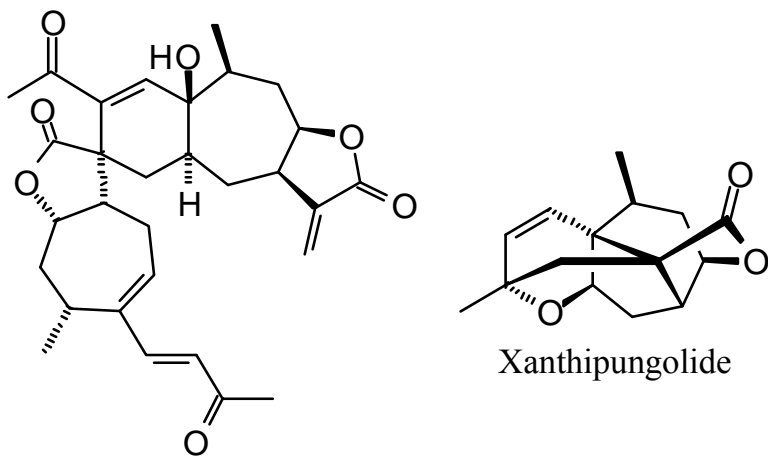

Xanthipungolide
8-Epixanthatin-1 $\beta, 5 \beta$ epoxide<smiles>C=C1C(=O)O[C@H]2C1[C@H](O)CC(C)=C1C(=O)C=C(CO)[C@H]12</smiles>

Lactucin

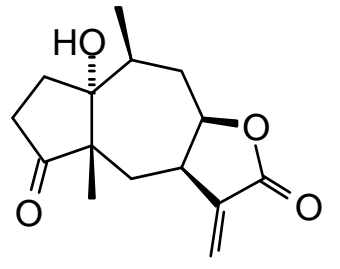

Peruvin<smiles></smiles>

Pungiolide A 
Figure 4. Eudesmanolide sesquiterpenoids examined in this work.<smiles>C=C1C(=O)OC2CC3(C)CCC[C@H](C)C3=CC12</smiles>

Alantolactone<smiles>C=C1C(=O)O[C@H]2[C@@H]1CCC1(C)CCCC(=C)[C@H]21</smiles>

$\beta$-Cyclocostunolide

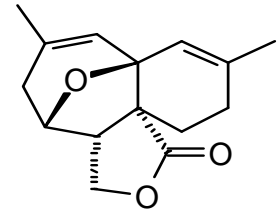

Anthecularin<smiles>C=C1CCC(O)[C@]2(C)C[C@@H]3OC(=O)C(=C)[C@H]3[C@H](O)C12</smiles>

Deacetyl- $\beta$ -

cyclopryethrosin<smiles>C=C1C(=O)O[C@@H]2C3=C(C)CCCC3(C)CC[C@@H]12</smiles>

Arbusculin B<smiles>C=C1C(=O)O[C@H]2C1CC[C@]1(C)[C@@H](O)CC=C[C@H]21</smiles>

Douglanin<smiles>CC1=CCCC2(C)CCC3C(=O)O[C@@H]3[C@H]12</smiles>

$\alpha$-Cyclocostunolide<smiles>C=C1C(=O)OC2C1=C(C)CCCC2(C)C</smiles>

Frullanolide<smiles>C=C[C@]12COC(=O)C(=C)C1[C@@H]1OC(=O)C(C)[C@H]1[C@@H](OC(=O)C(=C)CO)C2</smiles><smiles>C=C1C(=O)OCC1[C@@H](O)/C=C(\C)CC(=O)C=C(C)C</smiles>

4-Hydroxyanthecotulide

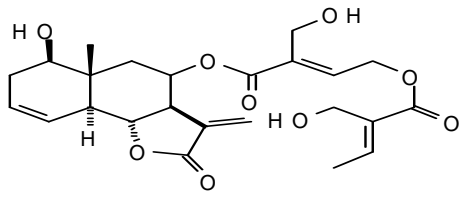

$8 \beta$-[4-Hydroxy-5-(5-hydroxytigloyloxy)tigloyl]santamarin
11,13-Dihydrovernodalin<smiles>C=C1C(=O)OC2CC3(C)CCCC(=C)[C@H]3CC12</smiles>

Isoalantolactone<smiles>C=C1C(=O)OC2C[C@@]3(C)CC(=O)C[C@H](C)C3=CC12</smiles>

2-Oxoalantolactone<smiles>CC1=C2[C@@H]3OC(=O)[C@@H](C)[C@H]3CCC2(C)C=CC1=O</smiles>

$\alpha-$ Santonin<smiles>C=C1OC2C[C@@]3(C)C[C@H](O)CC(=C)[C@@H]3CC2C1=C</smiles>

Ivalin<smiles>C=C1C(=O)OC2C[C@@]3(C)C[C@H](OC(C)=O)CC(=C)[C@H]3CC12</smiles>

Ivalin acetate<smiles>C=C1C2CC3C=C4OC(=O)C(CO)=C4C[C@@H]1C2C3</smiles>

Onoseriolide<smiles>C=C1C(=O)O[C@H]2C1CC[C@]1(C)C(O)CC=C(C)[C@H]21</smiles>

Santamarin<smiles>C=C(C)C(=O)OC1[C@H]2C(=C)C(=O)O[C@@H]2[C@H](C(C)=O)[C@@]2(C)[C@H](O)CC[C@](C)(O)[C@]12C</smiles>

Sivasinolide<smiles>C[C@H]1C(=O)O[C@H]2[C@@H]1CCC1(C)CCC(=O)C(O)(O)[C@@H]21</smiles>

4-Peroxy-1,2,4,5-tetrahydro-

Oxyonoseriolide<smiles>C=C1C(=O)O[C@H]2C[C@]3(C)C(O)CC=C(C)C3[C@@H](O)C12</smiles><smiles>C=C1C(=O)O[C@H]2[C@@H]1C(OC(=O)C(C)C)[C@H](OC(C)=O)[C@@]1(C)C(OC)CC[C@](C)(O)[C@@H]21</smiles>

Trilobolide 6-isobutyrate

Trilobolide 6-methacrylate<smiles>C=C[C@]1(COC)C[C@@H](OC(=O)C(=C)CO)[C@H]2C(=O)C(=O)O[C@@H]2[C@H]1C(=C)C=O</smiles>

Vernangulide B

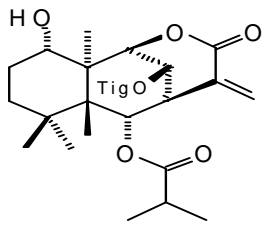

Wedelolide A<smiles>C=C[C@]12COC(=O)C(=C)[C@H]1[C@@H]1OC(=O)[C@H](C)[C@H]1[C@@H](OC(=O)C(=C)CO)C2</smiles>

Vernodalin<smiles>C=C[C@]12COC(=O)C(=C)[C@H]1[C@@H](O)C(C(=C)OC)[C@@H](OC(=O)C(=C)CO)C2</smiles>

Vernodalol

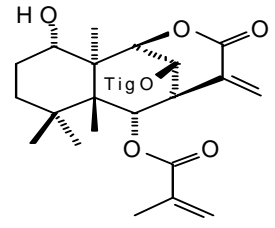

Wedelolide B 
Figure 5. Miscellaneous sesquiterpenoids examined in this work.

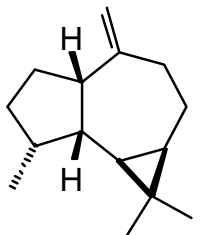

Alloaromadendrene<smiles>CC(C)C1CCC2(C)C(=O)CCC(C)C2(O)C1</smiles>

Corymbolone

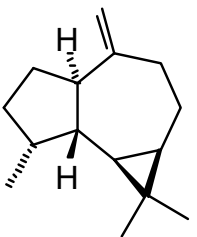

Aromadendrene<smiles>CC1=CCCC2(C)CCC(C(C)(C)O)C[C@H]12</smiles>

$\alpha$-Eudesmol

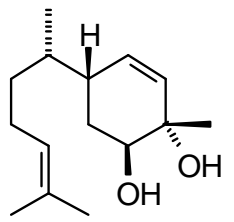

1,10-Bisaboladiene-3,4diol

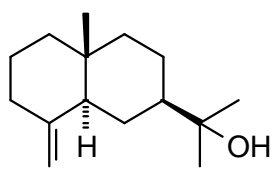

$\beta$-Eudesmol

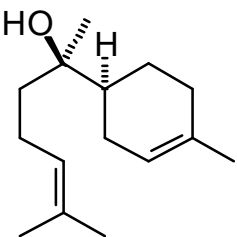

$\alpha$-Bisabolol<smiles>CC(C)C1CCC2=CCC[C@](C)(O)CC21</smiles>

1(10),5-Germacradien-4-ol<smiles>Cc1cc2c(C(C)C)c(O)c(O)c(C)c2c(O)c1-c1c(C)cc2c(C(C)C)c(O)c(O)c(C)c2c1O</smiles>

Gossypol<smiles>C=C1CCC=C2CCC(C(C)C)CC2C1</smiles>

Germacrene D<smiles>C=C(C)[C@@H]1CCC(CO)=C2CCC(C)[C@H]2C1</smiles>

14-Hydroperoxy1(10),11-guaiadiene<smiles>CC1(C)CCC[C@]2(O)C(C)(C)[C@@H](O)C=C(C=O)[C@]12C</smiles>

Mukaadial<smiles>CC(=O)[C@H]1CC[C@@H]2[C@@H](O)CCC(C(C)C)C12C</smiles>

Oplopanone<smiles>CC(C)=CCCC(C)C1CC2OC1CC2C</smiles>

Zingiberene-3,6- $\alpha$ peroxide

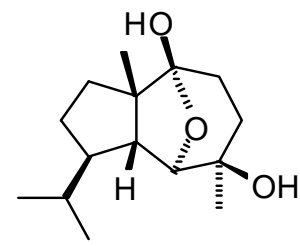

Homalomenol C<smiles>C=C1CCCC2(C)CCC([C@@](C)(O)CO)C[C@]12O</smiles>

Kudtriol<smiles>CC1=CC(=O)C2CC1C1CCC2C1C(C)C</smiles>

Mustakone<smiles>Cc1ccc2c(c1)C1CCC3(C)OOC2C13C</smiles>

10,12-Peroxycalamenene<smiles>CC(C)=CCCC(C)C1CCC2CCC1C2</smiles>

Zingiberene-3,6- $\beta$ peroxide<smiles>COc1c(O)c(C(C)C)c2c(O)c(-c3c(C)cc4c(C(C)C)c(O)c(O)c(C)c4c3O)c(C)cc2c1C=O</smiles>

Gossypol-6-methylether

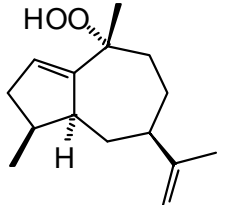

1-Hydroperoxy10(14),11-guaiadiene<smiles>C=C1CCCC2(C)CCC([C@@](C)(O)CO)CC12O</smiles>

5-epi-Kudtriol<smiles>C=C1C(C)CCC2(O)[C@H]1CC=C(C=O)C2(C)C</smiles>

Muzigadial<smiles>CC(=O)OCC1=CO[C@H](OC(C)=O)[C@H]2[C@@H]1[C@@H]1[C@H](CC[C@@]23CO3)C1(C)C</smiles>

Plagiochilin A
10-Hydroperoxy-1,11guaiadiene<smiles>CC1(C)CC2CC3CCC(C1)C3(Cl)C2</smiles>

Longifolene<smiles>C=CC(C)(O)CCC=C(C)CCC=C(C)C</smiles>

Nerolidol<smiles>CC1(C)CCCC2(C)[C@H](C=O)C(C=O)=CC[C@H]12</smiles>

Polygodial 
Table 4. MolDock docking energies $(\mathrm{kJ} / \mathrm{mol})$ of germacranolide sesquiterpenoids with Leishmania major protein targets.

\begin{tabular}{|c|c|c|c|c|c|c|c|c|c|c|c|c|}
\hline Germacranolides & LmajCatB & LmajDHODH & LmajdUTPase & LmajNDKb & LmajNH & LmajNMT & LmajOPB & LmajPDE1 & LmajPTR1 & LmajMetRS & LmajTyrRS & LmajUGPase \\
\hline 8-Acetyl-13-O-ethylpiptocarphol & -90.8 & -113.1 & -99.0 & -87.6 & -84.0 & -108.3 & -114.1 & -98.2 & -105.3 & -127.1 & -99.1 & -105.1 \\
\hline Centratherin & -85.4 & -115.1 & -92.2 & -95.7 & -96.6 & -106.8 & -101.2 & -102.8 & -101.5 & -125.8 & -112.9 & -108.0 \\
\hline Costunolide & -62.3 & -86.5 & -73.8 & -71.8 & -68.1 & -79.8 & -78.4 & -75.0 & -79.9 & -75.6 & -76.3 & -81.8 \\
\hline $\begin{array}{l}\text { 2-Deethoxy-2 } \beta \text { - } \\
\text { methoxyphantomolin }\end{array}$ & -94.9 & -121.1 & -96.1 & -85.7 & -88.2 & -95.4 & -96.6 & -101.1 & -102.4 & -114.6 & -105.2 & -95.3 \\
\hline 8,13-Diacetylpiptocarphol & -92.2 & -115.1 & -105.5 & -93.4 & -88.9 & -106.7 & -100.2 & -102.6 & -107.3 & -131.5 & -99.6 & -110.6 \\
\hline 16 6,17 -Dihydrobrachycalyxolide & -100.1 & -129.1 & -112.5 & -117.3 & -111.0 & -121.5 & -100.2 & -122.8 & -105.0 & -152.9 & -117.7 & -115.7 \\
\hline 11(13)-Dehydroivaxillin & -78.0 & -108.3 & -78.6 & -80.6 & -81.5 & -84.4 & -91.4 & -81.9 & -89.1 & -101.9 & -88.3 & -95.7 \\
\hline 11ß,13-Dihydrotanachin & -66.9 & -96.3 & -73.5 & -67.8 & -79.6 & -78.7 & -77.2 & -75.3 & -66.8 & -77.2 & -78.9 & -89.6 \\
\hline Elephantopin & -86.7 & -114.6 & -98.8 & -90.3 & -91.5 & -105.0 & -97.8 & -106.8 & -103.9 & -119.3 & -105.0 & -105.7 \\
\hline 11-epi-Ivaxillin & -76.9 & -109.9 & -80.5 & -81.7 & -83.5 & -85.1 & -94.9 & -86.9 & -90.1 & -102.4 & -80.7 & -85.9 \\
\hline $4,5 \alpha$-Epoxy- $6 \alpha$-acetoxy- & & & & & & & & & & & & \\
\hline $\begin{array}{l}\text { 1(10)E,11(13)-germacradien- } \\
12,8 \alpha \text {-olide }\end{array}$ & -77.1 & -92.4 & -94.2 & -80.4 & -82.5 & -99.6 & -95.4 & -89.1 & -86.5 & -94.8 & -87.9 & -92.5 \\
\hline $4 \alpha, 5 \beta$-Epoxy-8-epi-inunolide & -74.1 & -100.5 & -76.5 & -76.8 & -80.3 & -82.5 & -86.2 & -81.0 & -88.5 & -99.1 & -84.8 & -88.2 \\
\hline Eremantholide C & -89.2 & -103.4 & -86.5 & -100.2 & -86.2 & -96.6 & -95.3 & -98.1 & -83.1 & -100.9 & -87.2 & -101.8 \\
\hline Eupatoriopicrin & -83.7 & -107.8 & -106.2 & -99.2 & -104.9 & -114.5 & -111.1 & -105.7 & -100.3 & -120.7 & -97.3 & -102.0 \\
\hline Goyazensolide & -73.5 & -107.0 & -90.0 & -97.6 & -93.1 & -108.4 & -101.1 & -107.7 & -98.2 & -116.4 & -110.9 & -107.4 \\
\hline Hanphyllin & -71.2 & -91.5 & -73.8 & -76.9 & -77.7 & -80.8 & -79.5 & -76.3 & -80.7 & -85.7 & -84.5 & -84.4 \\
\hline $\begin{array}{l}\text { 8-(3'-Hydroxymethacryloxy)- } \\
\text { hirsutinolide 13-acetate }\end{array}$ & -94.0 & -107.3 & -106.8 & -105.6 & -104.4 & -107.8 & -105.0 & -110.7 & -109.6 & -134.9 & -105.1 & -108.6 \\
\hline Ineupatorolide A & -83.1 & -117.3 & -90.0 & -92.4 & -91.0 & -98.7 & -100.4 & -97.2 & -112.8 & -119.4 & -112.1 & -105.9 \\
\hline Ivaxillin & -76.6 & -104.2 & -79.3 & -78.3 & -75.2 & -83.2 & -90.6 & -82.6 & -83.4 & -98.1 & -82.8 & -88.3 \\
\hline Molephantin & -80.8 & -105.0 & -87.4 & -80.0 & -87.4 & -101.6 & -87.9 & -99.0 & -94.7 & -106.0 & -99.3 & -91.9 \\
\hline Neurolenin B & -86.0 & -116.4 & -88.0 & -100.0 & -88.7 & -95.6 & -77.3 & -103.0 & -86.7 & -99.8 & -90.3 & -99.5 \\
\hline Neurolenin C & -82.7 & -116.3 & -93.0 & -94.4 & -86.2 & -102.1 & -89.6 & -95.8 & -102.6 & -112.6 & -99.0 & -117.6 \\
\hline Neurolenin D & -90.5 & -111.1 & -95.9 & -99.2 & -92.4 & -97.5 & -87.9 & -101.4 & -94.6 & -104.7 & -90.7 & -100.6 \\
\hline Parthenolide & -65.8 & -98.7 & -80.6 & -75.2 & -81.4 & -78.8 & -83.1 & -80.1 & -84.4 & -89.3 & -79.3 & -89.6 \\
\hline Tagitinin $\mathrm{C}$ & -87.4 & -102.0 & -90.4 & -89.0 & -96.1 & -96.8 & -95.1 & -105.2 & -102.8 & -111.3 & -94.5 & -102.2 \\
\hline Tanachin & -71.3 & -94.1 & -71.1 & -78.3 & -76.4 & -80.6 & -82.5 & -78.9 & -75.1 & -91.4 & -80.0 & -86.8 \\
\hline Tatridin A & -74.1 & -96.6 & -69.7 & -75.7 & -76.3 & -80.7 & -88.6 & -78.4 & -89.8 & -91.2 & -80.8 & -90.7 \\
\hline 8-Tigloylhirsutinolide 13 -acetate & -93.8 & -116.4 & -105.4 & -98.0 & -99.9 & -114.6 & -110.3 & -113.6 & -113.6 & -124.8 & -105.5 & -119.9 \\
\hline Vernolide D & -97.8 & -124.5 & -110.9 & -106.3 & -121.8 & -117.9 & -112.4 & -118.3 & -114.4 & -128.1 & -111.8 & -121.5 \\
\hline
\end{tabular}


Table 5. MolDock docking energies ( $\mathrm{kJ} / \mathrm{mol})$ of germacranolide sesquiterpenoids with Leishmania donovani and L. mexicana protein targets.

\begin{tabular}{|c|c|c|c|c|c|c|c|c|c|c|c|c|}
\hline Germacranolides & LdonCatB & LdonCyp & LdonDHODH & LdonNMT & LmexGAPDH & LmexGPDH & LmexPGI & LmexPMM & $\begin{array}{c}\text { LmexPYK } \\
\text { Site } 1 \\
\end{array}$ & $\begin{array}{c}\text { LmexPYK } \\
\text { Site } 2 \\
\end{array}$ & $\begin{array}{c}\text { LmexPYK } \\
\text { Site } 2 \\
\end{array}$ & LmexTIM \\
\hline 8-Acetyl-13-O-ethylpiptocarphol & -92.9 & -97.6 & -79.4 & -84.2 & -93.2 & -99.2 & -88.6 & -113.3 & -102.5 & -94.9 & -99.2 & -95.1 \\
\hline Centratherin & -89.0 & -88.7 & -86.0 & -90.9 & -82.2 & -126.6 & -87.5 & -108.9 & -100.3 & -96.0 & -107.5 & -100.6 \\
\hline Costunolide & -78.9 & -80.5 & -61.1 & -65.8 & -63.5 & -80.6 & -64.5 & -76.0 & -84.8 & -73.8 & -87.1 & -77.6 \\
\hline $\begin{array}{l}\text { 2-Deethoxy-2 } \beta \text { - } \\
\text { methoxyphantomolin }\end{array}$ & -101.6 & -88.0 & -80.7 & -87.6 & -88.7 & -110.2 & -87.4 & -110.3 & -103.1 & -85.8 & -105.8 & -79.2 \\
\hline 8,13-Diacetylpiptocarphol & -86.4 & -99.7 & -88.0 & -92.8 & -96.2 & -102.6 & -97.4 & -118.1 & -103.0 & -100.4 & -94.7 & -100.0 \\
\hline 16 $\alpha, 17$-Dihydrobrachycalyxolide & -104.2 & -104.4 & -100.0 & -96.1 & -108.9 & -130.0 & -107.9 & -136.3 & -125.1 & -108.8 & -110.3 & -114.6 \\
\hline 11(13)-Dehydroivaxillin & -78.1 & -85.0 & -64.3 & -83.7 & -72.9 & -99.4 & -68.0 & -90.6 & -91.0 & -79.8 & -106.6 & -81.8 \\
\hline 11ß,13-Dihydrotanachin & -73.4 & -77.2 & -52.3 & -75.2 & -69.6 & -81.9 & -67.2 & -79.1 & -82.3 & -74.9 & -78.1 & -66.1 \\
\hline Elephantopin & -83.4 & -90.2 & -86.6 & -88.1 & -82.6 & -116.1 & -89.7 & -115.8 & -110.5 & -92.9 & -103.0 & -92.4 \\
\hline 11-epi-Ivaxillin & -77.0 & -84.8 & -66.5 & -88.4 & -74.1 & -96.3 & -66.4 & -88.9 & -93.2 & -79.4 & -106.9 & -78.0 \\
\hline 4,5 $\alpha$-Epoxy- $6 \alpha$-acetoxy- & & & & & & & & & & & & \\
\hline $\begin{array}{l}\text { 1(10)E,11(13)-germacradien- } \\
12,8 \alpha \text {-olide }\end{array}$ & -77.0 & -90.7 & -66.4 & -82.3 & -73.0 & -98.9 & -79.5 & -91.7 & -97.8 & -88.0 & -88.3 & -81.5 \\
\hline $4 \alpha, 5 \beta$-Epoxy-8-epi-inunolide & -73.3 & -85.8 & -76.9 & -78.8 & -67.6 & -90.7 & -62.3 & -86.8 & -87.8 & -75.2 & -95.4 & -78.6 \\
\hline Eremantholide C & -87.6 & -98.8 & -86.4 & -76.2 & -77.4 & -114.2 & -79.9 & -103.2 & -102.0 & -104.2 & -85.1 & -94.2 \\
\hline Eupatoriopicrin & -87.0 & -94.3 & -98.3 & -96.1 & -99.5 & -116.5 & -92.5 & -102.7 & -119.4 & -103.5 & -96.0 & -97.7 \\
\hline Goyazensolide & -86.2 & -85.7 & -83.9 & -82.3 & -87.8 & -124.1 & -88.4 & -107.9 & -99.0 & -95.9 & -103.4 & -98.7 \\
\hline Hanphyllin & -85.4 & -85.0 & -32.6 & -67.3 & -69.8 & -81.3 & -66.5 & -80.5 & -86.6 & -75.1 & -87.5 & -83.5 \\
\hline $\begin{array}{l}\text { 8-(3'-Hydroxymethacryloxy- } \\
\text { hirsutinolide } 13 \text {-acetate }\end{array}$ & -98.6 & -98.4 & -80.0 & -98.5 & -88.4 & -113.9 & -96.1 & -117.6 & -112.4 & -101.4 & -110.7 & -103.4 \\
\hline Ineupatorolide A & -97.4 & -95.2 & -94.2 & -86.5 & -72.5 & -102.4 & -82.2 & -99.7 & -100.1 & -91.2 & -100.4 & -104.6 \\
\hline Ivaxillin & -73.9 & -84.9 & -74.6 & -78.3 & -77.9 & -90.4 & -68.4 & -91.3 & -93.5 & -77.9 & -105.0 & -80.7 \\
\hline Molephantin & -87.9 & -94.5 & -36.3 & -89.4 & -79.4 & -92.9 & -78.8 & -96.6 & -106.8 & -88.8 & -99.8 & -99.9 \\
\hline Neurolenin B & -88.1 & -82.6 & -66.6 & -76.5 & -77.7 & -98.4 & -80.1 & -110.7 & -103.3 & -92.0 & -100.4 & -85.9 \\
\hline Neurolenin C & -93.6 & -89.8 & -49.0 & -94.7 & -86.5 & -94.4 & -79.3 & -106.0 & -95.0 & -89.9 & -91.6 & -95.2 \\
\hline Neurolenin D & -94.8 & -78.1 & -77.6 & -95.3 & -87.1 & -94.1 & -80.8 & -111.4 & -97.7 & -89.1 & -92.4 & -92.0 \\
\hline Parthenolide & -73.2 & -81.2 & -75.6 & -75.4 & -63.6 & -89.6 & -64.8 & -84.4 & -88.5 & -79.3 & -88.9 & -78.1 \\
\hline Tagitinin C & -88.0 & -84.6 & -52.0 & -87.5 & -85.2 & -92.8 & -78.0 & -112.3 & -101.4 & -91.5 & -98.0 & -91.4 \\
\hline Tanachin & -77.7 & -82.1 & -58.7 & -71.0 & -70.6 & -89.8 & -69.9 & -81.7 & -86.0 & -83.4 & -77.9 & -83.4 \\
\hline Tatridin A & -84.7 & -84.5 & -67.6 & -72.8 & -59.3 & -85.7 & -61.6 & -83.8 & -83.5 & -81.2 & -89.7 & -76.9 \\
\hline 8-Tigloylhirsutinolide 13-acetate & -104.5 & -83.9 & -94.8 & -97.3 & -91.0 & -110.3 & -99.2 & -117.5 & -111.3 & -98.3 & -108.0 & -102.8 \\
\hline Vernolide D & -107.5 & -94.3 & -89.6 & -102.3 & -94.8 & -120.3 & -96.8 & -125.4 & -117.4 & -113.7 & -99.8 & -109.1 \\
\hline
\end{tabular}


Table 6. MolDock docking energies $(\mathrm{kJ} / \mathrm{mol})$ of germacranolide sesquiterpenoids with Leishmania infantum protein targets.

\begin{tabular}{|c|c|c|c|c|c|}
\hline Germacranolides & LinfCYP51 & LinfGLO2 & LinfPnC1 & LinfTDR1 & LinfTR \\
\hline 8-Acetyl-13-O-ethylpiptocarphol & -100.4 & -89.8 & no dock & -82.3 & -99.7 \\
\hline Centratherin & -109.0 & -87.1 & no dock & -88.5 & -104.9 \\
\hline Costunolide & -74.4 & -63.6 & no dock & -69.9 & -71.2 \\
\hline 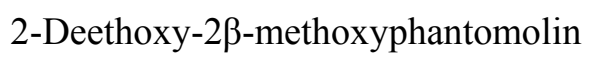 & -101.4 & -83.7 & no dock & -83.2 & -95.3 \\
\hline 8,13-Diacetylpiptocarphol & -97.8 & -88.8 & no dock & -91.7 & -101.6 \\
\hline $16 \alpha, 17$-Dihydrobrachycalyxolide & -126.4 & -108.8 & no dock & -104.2 & -112.9 \\
\hline 11(13)-Dehydroivaxillin & -81.4 & -69.4 & -86.5 & -79.4 & -81.2 \\
\hline 11 $\beta, 13$-Dihydrotanachin & -73.7 & -63.2 & -86.6 & -72.2 & -76.3 \\
\hline Elephantopin & -110.7 & -90.5 & no dock & -92.2 & -104.4 \\
\hline 11-epi-Ivaxillin & -83.4 & -73.9 & -79.8 & -77.7 & -81.2 \\
\hline 4,5 $\alpha$-Epoxy- $6 \alpha$-acetoxy- & & & & & \\
\hline $\begin{array}{l}\text { 1(10)E,11(13)-germacradien-12,8 } \alpha \text { - } \\
\text { olide }\end{array}$ & -92.9 & -79.5 & no dock & -80.9 & -89.6 \\
\hline $4 \alpha, 5 \beta$-Epoxy-8-epi-inunolide & -77.1 & -67.0 & -80.3 & -70.2 & -77.2 \\
\hline Eremantholide $\mathrm{C}$ & -91.3 & -70.7 & no dock & -81.6 & -95.3 \\
\hline Eupatoriopicrin & -100.1 & -91.3 & no dock & -96.5 & -105.1 \\
\hline Goyazensolide & -104.7 & -83.9 & no dock & -84.3 & -98.8 \\
\hline Hanphyllin & -79.3 & -64.9 & -64.6 & -75.7 & -76.4 \\
\hline $\begin{array}{l}\text { 8-(3'-Hydroxymethacryloxy)- } \\
\text { hirsutinolide } 13 \text {-acetate }\end{array}$ & -120.2 & -90.6 & no dock & -100.1 & -114.7 \\
\hline Ineupatorolide A & -102.5 & -84.2 & no dock & -89.1 & -95.0 \\
\hline Ivaxillin & -82.2 & -70.1 & no dock & -75.0 & -84.9 \\
\hline Molephantin & -101.0 & -82.4 & no dock & -82.3 & -101.2 \\
\hline Neurolenin B & -95.7 & -76.0 & no dock & -81.0 & -92.5 \\
\hline Neurolenin C & -95.9 & -76.9 & no dock & -82.0 & -93.3 \\
\hline Neurolenin D & -99.7 & -76.0 & no dock & -80.7 & -86.9 \\
\hline Parthenolide & -82.1 & -63.5 & -84.6 & -75.2 & -76.9 \\
\hline Tagitinin $\mathrm{C}$ & -97.0 & -79.4 & no dock & -77.6 & -90.7 \\
\hline Tanachin & -82.0 & -61.0 & -62.5 & -73.7 & -74.5 \\
\hline Tatridin A & -87.0 & -65.2 & -97.2 & -70.9 & -77.8 \\
\hline 8-Tigloylhirsutinolide 13 -acetate & -124.6 & -88.1 & no dock & -91.5 & -109.1 \\
\hline Vernolide D & -131.9 & -94.9 & no dock & -100.0 & -110.4 \\
\hline
\end{tabular}


Table 7. MolDock docking energies $(\mathrm{kJ} / \mathrm{mol})$ of guaianolide sesquiterpenoids with Leishmania major protein targets.

\begin{tabular}{|c|c|c|c|c|c|c|c|c|c|c|c|c|}
\hline Guaianolides & LmajCatB & LmajDHODH & LmajdUTPase & LmajNDKb & LmajNH & LmajNMT & LmajOPB & LmajPDE1 & LmajPTR1 & LmajMetRS & LmajTyrRS & LmajUGPase \\
\hline Arborescin & -66.2 & -93.6 & -78.3 & -83.0 & -72.7 & -78.2 & -85.3 & -77.7 & -95.1 & -90.4 & -91.8 & -79.5 \\
\hline Carpesiolin & -73.3 & -97.3 & -73.5 & -79.0 & -70.7 & -79.4 & -84.1 & -75.6 & -87.3 & -86.7 & -86.0 & -88.0 \\
\hline Confertin & -62.4 & -90.0 & -72.4 & -73.5 & -75.8 & -72.7 & -81.5 & -70.1 & -74.8 & -94.4 & -78.3 & -83.7 \\
\hline Cynaropicrin & -78.6 & -117.2 & -104.1 & -101.0 & -106.4 & -109.8 & -97.6 & -109.4 & -103.0 & -115.0 & -107.2 & -108.6 \\
\hline Damsin & -64.9 & -102.1 & -74.1 & -77.0 & -77.4 & -83.2 & -79.4 & -78.2 & -74.8 & -89.1 & -77.5 & -85.1 \\
\hline $\begin{array}{l}11,13- \\
\text { Dehydrocompressanolide }\end{array}$ & -72.6 & -94.1 & -77.6 & -78.6 & -74.9 & -76.8 & -78.7 & -80.1 & -85.0 & -93.3 & -81.7 & -92.6 \\
\hline Dehydrocostuslactone & -63.2 & -86.9 & -76.2 & -73.1 & -72.7 & -69.5 & -75.1 & -73.5 & -82.1 & -86.5 & -83.8 & -79.8 \\
\hline Dehydroleucodine & -66.9 & -89.5 & -85.2 & -84.2 & -80.6 & -79.9 & -83.7 & -84.2 & -94.2 & -90.9 & -93.3 & -83.2 \\
\hline Dehydrozaluzanin C & -73.3 & -90.4 & -74.9 & -78.7 & -69.8 & -79.0 & -86.3 & -77.7 & -85.4 & -92.2 & -88.4 & -85.4 \\
\hline Diguaiaperfolin & -127.8 & -154.5 & -130.6 & -116.8 & -135.7 & -129.4 & -114.3 & -129.7 & -124.3 & -129.9 & -144.6 & -151.8 \\
\hline 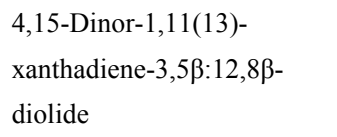 & -76.3 & -92.7 & -88.4 & -83.9 & -70.7 & -80.2 & -87.5 & -84.9 & -95.0 & -95.8 & -83.4 & -92.6 \\
\hline $\begin{array}{l}\text { 8-Epixanthatin- } 1 \beta, 5 \beta \text { - } \\
\text { epoxide }\end{array}$ & -78.0 & -108.0 & -77.8 & -89.0 & -84.5 & -88.3 & -87.1 & -88.3 & -92.0 & -106.8 & -89.5 & -95.4 \\
\hline Helenalin & -67.2 & -92.8 & -74.6 & -74.6 & -80.7 & -77.5 & -80.1 & -79.0 & -84.0 & -86.4 & -87.5 & -87.2 \\
\hline Helenalin acetate & -72.4 & -88.8 & -84.1 & -81.6 & -86.2 & -88.9 & -92.6 & -91.0 & -76.8 & -98.7 & -90.3 & -89.8 \\
\hline 8ß-Hydroxyzaluzanin D & -81.2 & -102.8 & -95.6 & -87.0 & -89.7 & -91.1 & -98.7 & -90.2 & -88.3 & -94.1 & -93.9 & -98.9 \\
\hline Lactucin & -68.8 & -100.7 & -84.2 & -91.9 & -93.4 & -87.9 & -89.7 & -91.7 & -104.1 & -92.2 & -103.6 & -89.3 \\
\hline Lactucopicrin & -97.7 & -123.3 & -113.8 & -107.4 & -110.9 & -112.6 & -116.8 & -122.0 & -129.9 & -137.7 & -116.5 & -120.1 \\
\hline Mexicanin I & -75.9 & -92.6 & -77.9 & -80.4 & -79.7 & -80.1 & -83.8 & -76.5 & -83.8 & -88.1 & -89.1 & -87.0 \\
\hline $\begin{array}{l}\text { 2-Oxo-8-tigloyloxyguaia- } \\
\text { 1(10),3-diene-6,12-olide-14- } \\
\text { carboxylic acid }\end{array}$ & -82.1 & -117.0 & -99.2 & -96.9 & -95.9 & -96.7 & -90.1 & -105.0 & -108.3 & -114.1 & -103.1 & -110.8 \\
\hline Peruvin & -62.0 & -91.6 & -71.8 & -72.0 & -78.4 & -81.3 & -79.5 & -76.2 & -79.1 & -85.4 & -78.2 & -101.9 \\
\hline Psilostachyin & -71.8 & -89.5 & -75.0 & -83.3 & -75.7 & -78.7 & -78.1 & -79.8 & -76.7 & -90.3 & -81.7 & -87.2 \\
\hline Psilostachyin C & -72.4 & -91.5 & -74.3 & -75.8 & -79.5 & -72.8 & -82.2 & -78.3 & -79.9 & -92.5 & -82.3 & -89.7 \\
\hline Pungiolide A & -91.6 & -96.7 & -96.8 & -116.1 & -106.0 & -109.6 & -111.8 & -122.8 & -108.3 & -122.6 & -107.3 & -117.5 \\
\hline Xanthipungolide & -52.4 & -78.4 & -50.0 & -55.4 & -62.7 & -64.5 & -62.6 & -66.8 & -48.3 & -69.8 & -60.4 & -74.8 \\
\hline Zaluzanin D & -68.6 & -103.0 & -93.1 & -86.7 & -80.0 & -93.1 & -90.8 & -93.7 & -89.1 & -96.5 & -97.1 & -95.3 \\
\hline
\end{tabular}


Table 8. MolDock docking energies $(\mathrm{kJ} / \mathrm{mol})$ of guaianolide sesquiterpenoids with Leishmania donovani and L. mexicana protein targets.

\begin{tabular}{|c|c|c|c|c|c|c|c|c|c|c|c|c|}
\hline Guaianolides & LdonCatB & LdonCyp & LdonDHODH & LdonNMT & LmexGAPDH & LmexGPDH & LmexPGI & LmexPMM & $\begin{array}{c}\text { LmexPYK } \\
\text { Site } 1 \\
\end{array}$ & $\begin{array}{c}\text { LmexPYK } \\
\text { Site } 2 \\
\end{array}$ & $\begin{array}{c}\text { LmexPYK } \\
\text { Site } 3 \\
\end{array}$ & LmexTIM \\
\hline Arborescin & -74.7 & -82.7 & -71.6 & -73.6 & -68.3 & --91.9 & -65.5 & -82.7 & -88.1 & -75.1 & -86.5 & -73.8 \\
\hline Carpesiolin & -76.7 & -78.5 & -61.5 & -69.7 & -66.5 & -91.8 & -61.9 & -83.4 & -85.7 & -88.6 & -94.1 & -82.1 \\
\hline Confertin & -67.1 & -85.2 & -12.0 & -68.1 & -62.1 & -85.1 & -61.5 & -79.0 & -85.9 & -77.1 & -82.1 & -70.8 \\
\hline Cynaropicrin & -80.5 & -99.7 & -57.3 & -92.8 & -88.0 & -121.8 & -97.2 & -114.7 & -127.0 & -91.8 & -94.1 & -90.1 \\
\hline Damsin & -73.3 & -88.7 & -0.9 & -73.6 & -69.4 & -85.7 & -64.6 & -79.5 & -88.1 & -79.9 & -84.8 & -68.3 \\
\hline 11,13-Dehydrocompressanolide & -74.2 & -87.8 & -73.4 & -72.8 & -70.3 & -93.1 & -64.9 & -86.0 & -84.0 & -82.1 & -92.3 & -79.6 \\
\hline Dehydrocostuslactone & -71.2 & -83.5 & -64.8 & -66.8 & -60.4 & -84.8 & -57.7 & -76.1 & -82.5 & -74.6 & -88.7 & -69.5 \\
\hline Dehydroleucodine & -78.0 & -84.2 & -63.9 & -72.1 & -73.5 & -88.0 & -66.2 & -83.0 & -91.4 & -74.7 & -89.7 & -72.3 \\
\hline Dehydrozaluzanin C & -74.4 & -86.1 & -79.0 & -76.0 & -68.3 & -91.0 & -70.8 & -82.9 & -85.4 & -80.5 & -99.5 & -77.9 \\
\hline Diguaiaperfolin & -126.1 & -124.8 & -121.6 & -135.5 & -122.1 & -138.1 & -129.7 & -145.6 & -146.9 & -141.4 & -138.3 & -116.5 \\
\hline 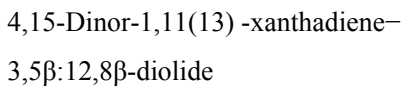 & -80.7 & -89.5 & -78.7 & -75.7 & -69.9 & -84.6 & -67.7 & -82.2 & -86.4 & -81.3 & -90.6 & -76.7 \\
\hline 8-Epixanthatin-1 $\beta, 5 \beta$-epoxide & -87.2 & -90.1 & -64.8 & -78.4 & -71.9 & -103.4 & -72.8 & -85.4 & -97.2 & -90.3 & -90.9 & -80.6 \\
\hline Helenalin & -70.7 & -90.8 & -47.7 & -76.1 & -69.5 & -79.3 & -64.9 & -80.8 & -83.1 & -85.2 & -83.6 & -79.0 \\
\hline Helenalin acetate & -74.2 & -77.2 & -76.9 & -74.9 & -78.5 & -105.5 & -74.7 & -87.6 & -99.3 & -86.9 & -78.5 & -79.9 \\
\hline $8 \beta$-Hydroxyzaluzanin D & -82.3 & -85.7 & -84.0 & -84.3 & -76.7 & -101.7 & -77.6 & -101.1 & -110.6 & -82.4 & -90.4 & -79.3 \\
\hline Lactucin & -87.0 & -87.4 & -70.5 & -72.2 & -84.2 & -94.7 & -74.0 & -92.4 & -100.7 & -87.8 & -94.2 & -79.1 \\
\hline Lactucopicrin & -114.3 & -111.2 & -65.0 & -114.4 & -99.5 & -126.7 & -108.5 & -119.9 & -120.4 & -115.0 & -116.1 & -107.3 \\
\hline Mexicanin I & -76.3 & -80.5 & -74.7 & -71.3 & -70.4 & -88.9 & -63.0 & -81.9 & -86.6 & -88.8 & -92.3 & -76.5 \\
\hline $\begin{array}{l}\text { 2-Oxo-8-tigloyloxyguaia-1(10),3- } \\
\text { diene-6,12-olide-14-carboxylic acid }\end{array}$ & -97.4 & -97.0 & -91.1 & -101.5 & -81.8 & -117.3 & -82.4 & -111.8 & -118.9 & -96.8 & -115.4 & -86.6 \\
\hline Peruvin & -66.7 & -83.6 & -64.1 & -74.6 & -62.7 & -83.0 & -61.5 & -83.0 & -88.5 & -79.1 & -83.6 & -74.1 \\
\hline Psilostachyin & -69.6 & -85.7 & -6.0 & -73.5 & -65.8 & -91.5 & -63.4 & -85.3 & -92.7 & -78.1 & -88.8 & -70.5 \\
\hline Psilostachyin C & -71.9 & -81.4 & -14.5 & -67.6 & -64.8 & -85.2 & -62.0 & -81.6 & -85.1 & -77.9 & -85.6 & -70.9 \\
\hline Pungiolide A & -97.3 & -102.9 & -83.7 & -93.9 & -89.1 & -110.0 & -98.8 & -105.0 & -123.5 & -111.6 & -81.4 & -98.4 \\
\hline Xanthipungolide & -50.4 & -63.3 & -3.8 & -55.4 & -57.3 & -75.0 & -43.1 & -67.5 & -66.9 & -57.2 & -83.7 & -54.3 \\
\hline Zaluzanin D & -83.6 & -87.2 & -59.0 & -79.4 & -77.1 & -102.6 & -75.1 & -96.2 & -106.8 & -83.6 & -97.7 & -86.4 \\
\hline
\end{tabular}


Table 9. MolDock docking energies $(\mathrm{kJ} / \mathrm{mol})$ of guaianolide sesquiterpenoids with Leishmania infantum protein targets.

\begin{tabular}{|c|c|c|c|c|c|}
\hline Guaianolides & LinfCYP51 & LinfGLO2 & LinfPnC1 & LinfTDR1 & LinfTR \\
\hline Arborescin & -79.3 & -64.1 & -59.5 & -70.7 & -95.1 \\
\hline Carpesiolin & -81.0 & -64.2 & -65.0 & -72.2 & -82.4 \\
\hline Confertin & -79.8 & -61.0 & -41.9 & -67.9 & -79.8 \\
\hline Cynaropicrin & -106.2 & -85.8 & no dock & -91.6 & -105.4 \\
\hline Damsin & -78.9 & -68.5 & -70.3 & -68.3 & -79.2 \\
\hline 11,13-Dehydrocompressanolide & -77.9 & -66.7 & -68.8 & -74.1 & -78.2 \\
\hline Dehydrocostuslactone & -73.5 & -61.4 & -68.8 & -69.1 & -82.4 \\
\hline Dehydroleucodine & -79.6 & -65.7 & -72.1 & -71.5 & -92.8 \\
\hline Dehydrozaluzanin C & -81.1 & -67.1 & -81.8 & -73.2 & -85.3 \\
\hline Diguaiaperfolin & -141.0 & -118.8 & no dock & -118.6 & -147.5 \\
\hline $\begin{array}{l}\text { 4,15-Dinor-1,11(13)-xanthadiene- } \\
3,5 \beta: 12,8 \beta \text {-diolide }\end{array}$ & -79.7 & -67.8 & -70.8 & -76.8 & -84.4 \\
\hline 8-Epixanthatin-1 $\beta, 5 \beta$-epoxide & -84.2 & -77.5 & -52.1 & -80.4 & -88.8 \\
\hline Helenalin & -89.0 & -62.4 & -24.6 & -70.1 & -79.7 \\
\hline Helenalin acetate & -85.9 & -71.7 & no dock & -76.1 & -81.6 \\
\hline $8 \beta$-Hydroxyzaluzanin D & -97.3 & -75.2 & no dock & -79.1 & -93.3 \\
\hline Lactucin & -88.9 & -72.7 & no dock & -85.0 & -99.8 \\
\hline Lactucopicrin & -114.8 & -103.6 & no dock & -106.7 & -101.6 \\
\hline Mexicanin I & -79.1 & -64.4 & -83.3 & -73.2 & -79.3 \\
\hline $\begin{array}{l}\text { 2-Oxo-8-tigloyloxyguaia-1(10),3- } \\
\text { diene-6,12-olide-14-carboxylic } \\
\text { acid }\end{array}$ & -100.1 & -94.1 & no dock & -92.0 & -100.8 \\
\hline Peruvin & -85.4 & -59.4 & no dock & -68.9 & -75.4 \\
\hline Psilostachyin & -85.7 & -69.7 & no dock & -67.2 & -84.5 \\
\hline Psilostachyin C & -74.1 & -59.9 & -55.5 & -67.0 & -82.1 \\
\hline Pungiolide A & -109.4 & -97.5 & -69.9 & -105.6 & -114.8 \\
\hline Xanthipungolide & -64.8 & -52.0 & -32.1 & -47.9 & -59.0 \\
\hline Zaluzanin D & -92.4 & -75.7 & no dock & -77.4 & -86.5 \\
\hline
\end{tabular}


Table 10. MolDock docking energies ( $\mathrm{kJ} / \mathrm{mol})$ of eudesmanolide sesquiterpenoids with Leishmania major protein targets.

\begin{tabular}{|c|c|c|c|c|c|c|c|c|c|c|c|c|}
\hline Eudesmanolides & LmajCatB & LmajDHODH & LmajdUTPase & LmajNDKb & LmajNH & LmajNMT & LmajOPB & LmajPDE1 & LmajPTR1 & LmajMetRS & LmajTyrRS & LmajUGPase \\
\hline Alantolactone & -58.1 & -90.7 & -61.4 & -79.0 & -73.8 & -72.4 & -70.1 & -67.2 & -65.8 & -83.2 & -74.2 & -87.9 \\
\hline Anthecularin & -56.1 & -77.7 & -62.7 & -68.7 & -67.2 & -65.7 & -73.7 & -72.3 & -59.6 & -70.9 & -72.6 & -85.9 \\
\hline Arbusculin B & -54.8 & -79.8 & -67.6 & -64.4 & -66.2 & -71.8 & -75.8 & -64.1 & -66.6 & -75.9 & -73.3 & -79.8 \\
\hline$\alpha-$ Cyclocostunolide & -63.7 & -85.3 & -75.1 & -72.6 & -69.7 & -70.6 & -74.7 & -69.1 & -78.5 & -86.1 & -77.1 & -77.2 \\
\hline$\beta$-Cyclocostunolide & -64.6 & -85.9 & -67.0 & -73.5 & -67.9 & -69.7 & -76.1 & -66.7 & -81.9 & -83.2 & -73.0 & -77.9 \\
\hline Deacetyl- $\beta$-cyclopryethrosin & -69.1 & -97.8 & -73.4 & -76.4 & -72.9 & -80.2 & -82.3 & -76.9 & -83.3 & -93.6 & -76.0 & -91.1 \\
\hline 11,13-Dihydrovernodalin & -78.0 & -110.6 & -91.9 & -100.3 & -89.7 & -99.7 & -99.0 & -106.2 & -103.4 & -114.7 & -104.0 & -94.5 \\
\hline Douglanin & -66.7 & -90.4 & -76.9 & -71.6 & -71.9 & -69.6 & -77.8 & -74.7 & -79.4 & -88.2 & -77.1 & -78.5 \\
\hline Frullanolide & -60.6 & -81.1 & -71.3 & -73.2 & -69.2 & -73.1 & -76.9 & -77.3 & -73.5 & -80.4 & -75.1 & -82.6 \\
\hline 4-Hydroxyanthecotulide & -76.1 & -104.1 & -90.1 & -103.0 & -88.3 & -101.7 & -95.0 & -100.5 & -100.8 & -103.2 & -98.1 & -112.3 \\
\hline $\begin{array}{l}8 \beta-[4-\text { Hydroxy-5-(5-hydroxytigloyloxy)- } \\
\text { tigloyl] santamarin }\end{array}$ & -109.5 & -128.9 & -116.3 & -125.3 & -117.8 & -115.9 & -113.3 & -117.5 & -130.2 & -153.1 & -128.3 & -127.1 \\
\hline Isoalantolactone & -60.2 & -89.6 & -65.0 & -82.0 & -74.4 & -74.4 & -70.3 & -72.2 & -66.4 & -87.3 & -74.2 & -88.6 \\
\hline Ivalin & -66.1 & -94.6 & -68.6 & -78.4 & -74.3 & -80.7 & -74.8 & -74.6 & -73.3 & -88.8 & -73.7 & -92.0 \\
\hline Ivalin acetate & -77.4 & -93.6 & -87.4 & -87.4 & -83.2 & -89.5 & -90.8 & -89.6 & -91.6 & -104.5 & -87.8 & -83.4 \\
\hline Onoseriolide & -72.4 & -92.4 & -82.8 & -81.4 & -78.5 & -79.8 & -88.5 & -83.8 & -88.6 & -101.4 & -81.3 & -82.5 \\
\hline 2-Oxoalantolactone & -59.1 & -94.0 & -64.8 & -79.6 & -76.3 & -78.0 & -75.0 & -69.7 & -68.4 & -85.5 & -75.0 & -91.1 \\
\hline Oxyonoseriolide & -77.5 & -94.5 & -82.3 & -80.4 & -81.5 & -88.9 & -88.7 & -88.0 & -87.8 & -104.7 & -92.7 & -99.1 \\
\hline 4-Peroxy-1,2,4,5-tetrahydro- $\alpha$-santonin & -63.3 & -97.6 & -72.6 & -71.0 & -72.5 & -74.1 & -87.3 & -80.3 & -90.1 & -85.2 & -76.6 & -82.4 \\
\hline Santamarin & -65.3 & -86.0 & -74.9 & -72.7 & -72.6 & -71.6 & -80.5 & -73.1 & -79.0 & -89.3 & -79.4 & -79.4 \\
\hline$\alpha-$ Santonin & -63.8 & -92.0 & -73.1 & -73.4 & -68.6 & -71.3 & -84.1 & -78.0 & -82.7 & -85.6 & -85.1 & -84.1 \\
\hline Sivasinolide & -69.8 & -90.0 & -68.9 & -84.5 & -72.9 & -78.7 & -84.5 & -72.6 & -86.8 & -98.1 & -80.6 & -82.9 \\
\hline Trilobolide 6-isobutyrate & -86.0 & -108.8 & -90.3 & -93.8 & -95.6 & -103.7 & -102.2 & -97.6 & -82.6 & -87.4 & -95.1 & -94.7 \\
\hline Trilobolide 6-methacrylate & -78.4 & -103.8 & -87.9 & -85.0 & -94.2 & -111.3 & -87.6 & -97.6 & -78.9 & -87.5 & -92.1 & -92.2 \\
\hline Vernangulide B & -91.2 & -118.3 & -108.7 & -105.8 & -105.6 & -113.0 & -99.8 & -107.1 & -122.9 & -125.3 & -105.0 & -111.9 \\
\hline Vernodalin & -80.8 & -107.5 & -94.4 & -101.0 & -92.4 & -105.7 & -89.4 & -92.4 & -104.5 & -116.3 & -95.8 & -102.2 \\
\hline Vernodalol & -82.5 & -112.8 & -100.1 & -99.1 & -96.4 & -97.3 & -94.5 & -99.4 & -100.0 & -107.8 & -94.6 & -90.2 \\
\hline Wedelolide A & -88.3 & -109.9 & -95.0 & -83.5 & -94.0 & -109.6 & -84.2 & -103.7 & -83.1 & -131.6 & -96.2 & -98.4 \\
\hline Wedelolide B & -88.0 & -113.0 & -91.2 & -87.0 & -94.2 & -107.4 & -99.0 & -107.4 & -82.8 & -134.0 & -102.8 & -94.9 \\
\hline
\end{tabular}


Table 11. MolDock docking energies $(\mathrm{kJ} / \mathrm{mol})$ of eudesmanolide sesquiterpenoids with Leishmania donovani and L. mexicana protein targets.

\begin{tabular}{|c|c|c|c|c|c|c|c|c|c|c|c|c|}
\hline Eudesmanolides & LdonCatB & LdonCyp & LdonDHODH & LdonNMT & LmexGAPDH & LmexGPDH & LmexPGI & LmexPMM & $\begin{array}{r}\text { LmexPYK } \\
\text { Site } 1 \\
\end{array}$ & $\begin{array}{r}\text { LmexPYK } \\
\text { Site } 2 \\
\end{array}$ & $\begin{array}{r}\text { LmexPYK } \\
\text { Site } 3 \\
\end{array}$ & LmexTIM \\
\hline Alantolactone & -67.1 & -78.3 & -55.0 & -64.4 & -62.6 & -79.8 & -57.8 & -74.6 & -78.6 & -72.2 & -79.7 & -77.9 \\
\hline Anthecularin & -62.4 & -65.2 & -57.0 & -63.9 & -60.1 & -80.3 & -59.8 & -70.9 & -79.7 & -64.4 & -81.3 & -69.7 \\
\hline Arbusculin B & -60.0 & -74.6 & -58.2 & -63.5 & -64.3 & -84.1 & -55.6 & -75.3 & -73.9 & -69.6 & -74.8 & -70.3 \\
\hline$\alpha$-Cyclocostunolide & -67.2 & -78.6 & -62.6 & -62.9 & -60.5 & -83.6 & -57.3 & -73.7 & -78.8 & -73.8 & -80.1 & -68.0 \\
\hline$\beta$-Cyclocostunolide & -69.9 & -78.0 & -69.9 & -67.0 & -60.2 & -85.0 & -58.9 & -78.3 & -76.3 & -75.2 & -87.9 & -73.4 \\
\hline Deacetyl- $\beta$-cyclopryethrosin & -73.4 & -82.9 & -63.3 & -72.8 & -71.5 & -92.2 & -63.3 & -84.0 & -84.6 & -78.1 & -88.1 & -81.4 \\
\hline 11,13-Dihydrovernodalin & -89.3 & -94.8 & -59.5 & -84.3 & -79.3 & -107.0 & -82.3 & -102.5 & -104.4 & -94.9 & -100.7 & -87.1 \\
\hline Douglanin & -69.0 & -80.6 & -71.4 & -66.3 & -63.7 & -88.6 & -58.9 & -76.6 & -82.7 & -77.2 & -82.1 & -65.1 \\
\hline Frullanolide & -61.5 & -77.8 & -65.2 & -63.1 & -67.0 & -79.4 & -58.7 & -75.6 & -82.1 & -72.5 & -86.1 & -72.8 \\
\hline 4-Hydroxyanthecotulide & -87.9 & -94.0 & -93.9 & -86.1 & -86.4 & -103.7 & -85.0 & -100.3 & -98.8 & -91.5 & -98.0 & -97.0 \\
\hline $\begin{array}{l}8 \beta-[4-\text { Hydroxy-5-(5-hydroxytigloyloxy)- } \\
\text { tigloyl] santamarin }\end{array}$ & -112.4 & -115.3 & -37.4 & -121.4 & -96.5 & -128.2 & -113.8 & -132.1 & -124.3 & -119.9 & -112.8 & -105.4 \\
\hline Isoalantolactone & -69.9 & -83.1 & -70.6 & -64.4 & -59.3 & -82.4 & -56.8 & -77.4 & -80.0 & -69.7 & -85.1 & -74.0 \\
\hline Ivalin & -77.3 & -84.5 & -74.6 & -73.2 & -62.5 & -84.1 & -62.0 & -80.9 & -83.6 & -73.4 & -88.9 & -70.0 \\
\hline Ivalin acetate & -91.5 & -92.5 & -63.2 & -66.9 & -73.8 & -97.0 & -70.5 & -92.4 & -97.2 & -80.3 & -81.4 & -86.8 \\
\hline Onoseriolide & -73.8 & -88.1 & -84.8 & -77.0 & -72.8 & -93.9 & -66.8 & -84.1 & -87.9 & -78.0 & -95.0 & -77.1 \\
\hline 2-Oxoalantolactone & -64.0 & -80.8 & -58.1 & -64.1 & -65.3 & -85.5 & -61.4 & -77.8 & -81.7 & -74.7 & -83.5 & -77.9 \\
\hline Oxyonoseriolide & -77.9 & -84.5 & -61.8 & -78.5 & -78.5 & -95.4 & -70.4 & -84.6 & -98.4 & -80.6 & -103.9 & -79.3 \\
\hline 4-Peroxy-1,2,4,5-tetrahydro- $\alpha$-santonin & -68.0 & -72.4 & -68.6 & -72.3 & -62.0 & -92.4 & -58.1 & -82.3 & -85.2 & -73.3 & -83.0 & -73.0 \\
\hline Santamarin & -69.3 & -79.8 & -56.3 & -67.4 & -65.6 & -89.5 & -61.2 & -81.2 & -82.6 & -75.9 & -82.0 & -71.6 \\
\hline$\alpha$-Santonin & -68.9 & -78.3 & -71.0 & -68.6 & -65.5 & -85.6 & -57.8 & -82.5 & -80.7 & -69.1 & -85.4 & -72.0 \\
\hline Sivasinolide & -71.6 & -85.1 & -61.8 & -73.5 & -68.1 & -94.9 & -62.3 & -84.7 & -88.4 & -77.9 & -91.5 & -76.4 \\
\hline Trilobolide 6-isobutyrate & -82.4 & -83.4 & -71.3 & -80.7 & -79.8 & -99.2 & -84.5 & -103.2 & -96.5 & -89.4 & -92.1 & -72.4 \\
\hline Trilobolide 6-methacrylate & -83.4 & -80.8 & -20.7 & -82.7 & -70.3 & -99.2 & -88.3 & -107.3 & -94.9 & -92.7 & -87.2 & -74.7 \\
\hline Vernangulide B & -93.8 & -106.6 & -126.8 & -96.1 & -90.5 & -121.5 & -100.6 & -120.1 & -116.6 & -105.6 & -107.5 & -104.7 \\
\hline Vernodalin & -84.6 & -94.2 & -79.2 & -87.4 & -80.6 & -110.8 & -81.5 & -106.2 & -104.5 & -106.1 & -99.5 & -94.0 \\
\hline Vernodalol & -88.4 & -96.3 & -50.6 & -83.9 & -84.1 & -105.2 & -93.5 & -99.8 & -103.5 & -99.1 & -103.4 & -83.2 \\
\hline Wedelolide A & -87.9 & -68.5 & -85.5 & -91.6 & -84.0 & -96.0 & -90.3 & -114.9 & -103.2 & -107.4 & -92.9 & -92.7 \\
\hline Wedelolide B & -81.5 & -64.3 & -88.3 & -97.5 & -79.2 & -96.0 & -92.2 & -127.4 & -102.4 & -108.8 & -102.6 & -96.0 \\
\hline
\end{tabular}


Table 12. MolDock docking energies $(\mathrm{kJ} / \mathrm{mol})$ of eudesmanolide sesquiterpenoids with Leishmania infantum protein targets.

\begin{tabular}{|c|c|c|c|c|c|}
\hline Eudesmanolides & LinfCYP51 & LinfGLO2 & LinfPnC1 & LinfTDR1 & LinfTR \\
\hline Alantolactone & -71.3 & -60.0 & no dock & -66.1 & -75.5 \\
\hline Anthecularin & -70.8 & -47.5 & -58.4 & -62.3 & -71.0 \\
\hline Arbusculin B & -71.0 & -58.8 & -12.7 & -62.1 & -73.6 \\
\hline$\alpha-$ Cyclocostunolide & -70.4 & -60.3 & -82.7 & -69.1 & -79.0 \\
\hline$\beta-$ Cyclocostunolide & -75.9 & -62.0 & -79.0 & -71.6 & -73.1 \\
\hline$\gamma$-Cyclocostunolide & -71.0 & -57.5 & -12.1 & -62.2 & -67.4 \\
\hline Deacetyl- $\beta-$ cyclopryethrosin & -74.8 & -64.8 & -75.5 & -71.5 & -82.2 \\
\hline 11,13-Dihydrovernodalin & -105.4 & -83.0 & no dock & -85.8 & -95.4 \\
\hline Douglanin & -76.4 & -60.7 & -10.6 & -67.9 & -80.1 \\
\hline Frullanolide & -75.2 & -61.3 & -55.4 & -70.5 & -74.5 \\
\hline 4-Hydroxyanthecotulide & -90.2 & -82.6 & -68.4 & -85.4 & -92.1 \\
\hline $\begin{array}{l}8 \beta-[4-\text { Hydroxy }-5-(5-\text { hydroxytigloyloxy })- \\
\text { tigloyl]santamarin }\end{array}$ & -117.8 & -98.9 & no dock & -103.4 & -114.9 \\
\hline Isoalantolactone & -69.1 & -57.8 & -31.1 & -64.3 & -74.2 \\
\hline Ivalin & -72.9 & -65.0 & -16.1 & -65.6 & -80.5 \\
\hline Ivalin acetate & -88.7 & -72.0 & no dock & -76.6 & -91.2 \\
\hline Onoseriolide & -82.0 & -70.0 & -24.6 & -71.8 & -82.4 \\
\hline 2-Oxoalantolactone & -73.4 & -57.6 & no dock & -68.1 & -79.4 \\
\hline Oxyonoseriolide & -86.7 & -71.3 & -11.8 & -78.4 & -87.9 \\
\hline 4-Peroxy-1,2,4,5-tetrahydro- $\alpha$-santonin & -80.6 & -73.2 & -66.0 & -66.5 & -78.2 \\
\hline Santamarin & -67.8 & -59.5 & -85.2 & -71.3 & -81.3 \\
\hline$\alpha-$ Santonin & -74.2 & -69.4 & -44.0 & -65.5 & -86.4 \\
\hline Sivasinolide & -75.2 & -63.3 & -72.1 & -72.5 & -81.1 \\
\hline Trilobide 6-isobutyrate & -97.9 & -82.0 & no dock & -80.3 & -90.2 \\
\hline Trilobide 6-methacrylate & -96.0 & -86.3 & no dock & -77.2 & -90.2 \\
\hline Vernangulide B & -105.2 & -98.6 & no dock & -99.3 & -99.0 \\
\hline Vernodalin & -105.9 & -88.9 & no dock & -86.6 & -96.9 \\
\hline Vernodalol & -103.1 & -79.4 & no dock & -88.9 & -96.8 \\
\hline Wedelolide A & -97.6 & -80.8 & no dock & -90.4 & -99.4 \\
\hline Wedelolide B & -105.8 & -77.6 & no dock & -92.8 & -96.6 \\
\hline
\end{tabular}


Table 13. MolDock docking energies (kJ/mol) of miscellaneous sesquiterpenoids with Leishmania major protein targets.

\begin{tabular}{|c|c|c|c|c|c|c|c|c|c|c|c|c|}
\hline Miscellaneous Sesquiterpenoids & LmajCatB & LmajDHODH & LmajdUTPase & LmajNDKb & LmajNH & LmajNMT & LmajOPB & LmajPDE1 & LmajPTR1 & LmajMetRS & LmajTyrRS & LmajUGPase \\
\hline Alloaromadendrene & -56.8 & -81.7 & -67.0 & -68.2 & -66.1 & -71.1 & -74.3 & -68.2 & -91.9 & -70.7 & -70.0 & -76.6 \\
\hline Aromadendrene & -56.9 & -78.5 & -68.4 & -66.9 & -64.7 & -70.2 & -73.2 & -67.9 & -99.4 & -73.2 & -72.4 & -72.2 \\
\hline 1,10-Bisaboladiene-3,4-diol & -67.4 & -92.7 & -81.0 & -82.8 & -79.8 & -87.5 & -89.2 & -82.9 & -102.6 & -91.1 & -80.7 & -89.5 \\
\hline$\alpha$-Bisabolol & -75.2 & -92.5 & -76.3 & -77.5 & -75.6 & -89.9 & -74.7 & -79.9 & -110.6 & -93.8 & -80.3 & -83.2 \\
\hline Corymbolone & -56.5 & -82.2 & -59.1 & -67.5 & -70.2 & -66.5 & -69.5 & -65.5 & -59.5 & -77.2 & -68.3 & -85.9 \\
\hline$\alpha$-Eudesmol & -60.9 & -80.3 & -64.8 & -70.7 & -68.1 & -66.0 & -66.0 & -74.2 & -86.8 & -83.1 & -76.9 & -74.7 \\
\hline$\beta$-Eudesmol & -58.8 & -83.4 & -69.6 & -69.6 & -66.5 & -68.2 & -72.7 & -67.3 & -89.6 & -76.9 & -68.1 & -79.1 \\
\hline 1(10),5-Germacradien-4-ol & -62.0 & -88.1 & -68.8 & -69.1 & -73.3 & -70.1 & -76.0 & -72.2 & -100.0 & -87.1 & -75.0 & -88.6 \\
\hline Germacrene D & -57.9 & -81.4 & -66.6 & -65.1 & -70.8 & -68.8 & -69.7 & -69.9 & -96.7 & -77.7 & -70.6 & -81.8 \\
\hline Gossypol & -59.1 & -106.1 & -85.2 & -89.5 & -83.9 & -104.6 & -98.4 & -111.5 & -120.6 & -92.7 & -108.0 & -90.7 \\
\hline Gossypol-6,6'-dimethylether & -90.8 & -108.3 & -84.1 & -95.0 & -85.6 & -95.5 & -84.6 & -114.3 & -117.5 & -88.0 & -108.6 & -100.3 \\
\hline Gossypol-6-methylether & -93.1 & -109.1 & -85.9 & -93.7 & -103.2 & -116.8 & -102.1 & -113.3 & -122.2 & -94.1 & -111.6 & -95.6 \\
\hline Homalomenol C & -67.8 & -88.7 & -65.1 & -69.4 & -72.6 & -72.4 & -76.6 & -74.7 & -92.6 & -83.9 & -75.5 & -88.6 \\
\hline 1-Hydroperoxy-10(14),11-guaiadiene & -60.7 & -85.4 & -70.4 & -70.7 & -69.0 & -74.0 & -77.5 & -78.5 & -96.5 & -83.6 & -76.5 & -83.7 \\
\hline 10-Hydroperoxy-1,11-guaiadiene & -64.6 & -82.2 & -76.5 & -73.3 & -74.6 & -77.5 & -77.5 & -82.7 & -109.1 & -86.4 & -80.1 & -79.9 \\
\hline 14-Hydroperoxy-1(10),11-guaiadiene & -71.5 & -88.0 & -88.1 & -79.2 & -78.4 & -78.5 & -80.0 & -82.3 & -111.6 & -87.9 & -79.1 & -86.6 \\
\hline Kudtriol & -60.6 & -86.7 & -65.3 & -68.9 & -72.5 & -71.5 & -66.2 & -69.2 & -81.5 & -82.3 & -68.0 & -80.5 \\
\hline 5-epi-Kudtriol & -59.9 & -86.5 & -69.2 & -68.4 & -70.4 & -78.2 & -75.8 & -75.8 & -65.9 & -77.9 & -65.1 & -83.0 \\
\hline Longifolene & -52.1 & -75.8 & -50.0 & -62.3 & -59.6 & -58.9 & -63.0 & -66.7 & -61.3 & -62.3 & -63.1 & -70.8 \\
\hline Mukaadial & -67.0 & -89.5 & -71.1 & -72.7 & -67.3 & -75.0 & -76.6 & -72.0 & -80.7 & -79.2 & -82.3 & -81.8 \\
\hline Mustakone & -47.5 & -78.0 & -61.9 & -62.0 & -64.5 & -64.2 & -67.6 & -67.5 & -66.1 & -71.7 & -65.4 & -81.7 \\
\hline Muzigadial & -64.6 & -93.9 & -66.8 & -70.6 & -65.0 & -70.6 & -74.1 & -66.3 & -81.5 & -81.9 & -68.5 & -76.8 \\
\hline Nerolidol & -69.5 & -91.4 & -86.4 & -86.5 & -81.2 & -93.0 & -84.3 & -89.8 & -117.0 & -100.5 & -87.9 & -94.2 \\
\hline Oplopanone & -59.4 & -81.9 & -69.4 & -66.1 & -68.2 & -70.8 & -73.0 & -71.7 & -106.4 & -76.9 & -74.0 & -74.2 \\
\hline 10,12-Peroxycalamenene & -41.0 & -71.0 & -57.9 & -57.4 & -60.8 & -65.9 & -66.3 & -74.3 & -70.6 & -69.3 & -68.4 & -70.7 \\
\hline Plagiochilin A & -83.4 & -103.9 & -94.1 & -94.1 & -93.6 & -88.5 & -95.7 & -92.7 & -132.3 & -102.9 & -106.0 & -105.9 \\
\hline Polygodial & -61.3 & -86.0 & -70.7 & -71.6 & -64.8 & -71.0 & -76.9 & -66.6 & -91.1 & -86.3 & -71.5 & -80.4 \\
\hline Zingiberene $-3,6-\alpha-$ peroxide & -62.3 & -89.4 & -75.8 & -75.4 & -76.0 & -82.6 & -68.3 & -84.9 & -87.4 & -92.0 & -76.8 & -88.2 \\
\hline Zingiberene $-3,6-\beta-$ peroxide & -62.2 & -86.9 & -73.6 & -75.4 & -74.9 & -78.7 & -72.4 & -84.7 & -89.9 & -82.0 & -73.7 & -84.9 \\
\hline
\end{tabular}


Table 14. MolDock docking energies ( $\mathrm{kJ} / \mathrm{mol})$ of miscellaneous sesquiterpenoids with Leishmania donovani and L. mexicana protein targets.

\begin{tabular}{|c|c|c|c|c|c|c|c|c|c|c|c|c|}
\hline Miscellaneous Sesquiterpenoids & LdonCatB & LdonCyp & LdonDHODH & LdonNMT & LmexGAPDH & LmexGPDH & LmexPGI & LmexPMM & LmexPYK & LmexPYK & LmexPYK & LmexTIM \\
\hline & & & & & & & & & Site 1 & Site 2 & Site 3 & \\
\hline Alloaromadendrene & -66.6 & -77.8 & -46.3 & -58.8 & -61.3 & -78.3 & -57.1 & -71.4 & -79.0 & -62.3 & -79.7 & -68.9 \\
\hline Aromadendrene & -66.1 & -72.0 & -56.6 & -62.4 & -57.1 & -75.0 & -54.4 & -71.9 & -79.4 & -65.5 & -77.1 & -65.1 \\
\hline 1,10-Bisaboladiene-3,4-diol & -73.0 & -82.3 & -74.6 & -85.5 & -70.9 & -94.2 & -71.9 & -78.6 & -88.2 & -76.9 & -84.5 & -80.0 \\
\hline$\alpha-$ Bisabolol & -75.2 & -76.8 & -68.1 & -81.0 & -65.2 & -85.2 & -67.0 & -84.8 & -90.3 & -75.7 & -79.7 & -77.7 \\
\hline Corymbolone & -60.9 & -72.2 & -62.0 & -63.5 & -60.6 & -74.4 & -56.1 & -70.5 & -74.6 & -72.4 & -80.2 & -69.4 \\
\hline $6 \alpha, 9 \alpha$-Dihydroxypolygodial & -65.8 & -86.7 & -61.9 & -72.3 & -65.5 & -88.4 & -58.7 & -77.1 & -83.6 & -74.4 & -91.4 & -69.6 \\
\hline$\alpha$-Eudesmol & -62.3 & -74.4 & -49.2 & -57.4 & -57.2 & -78.4 & -53.9 & -76.3 & -73.5 & -64.3 & -82.9 & -67.2 \\
\hline$\beta$-Eudesmol & -66.1 & -74.1 & -57.0 & -63.4 & -58.8 & -75.1 & -56.5 & -77.8 & -72.4 & -65.5 & -83.0 & -68.3 \\
\hline 1(10),5-Germacradien-4-ol & -69.7 & -78.5 & -49.2 & -70.5 & -62.2 & -81.5 & -58.6 & -75.2 & -78.1 & -68.4 & -84.1 & -74.8 \\
\hline Germacrene D & -65.0 & -74.6 & -64.4 & -68.3 & -60.1 & -77.0 & -58.3 & -70.1 & -73.5 & -64.9 & -82.0 & -71.3 \\
\hline Gossypol & -79.2 & -86.4 & -88.4 & -64.5 & -81.7 & -117.4 & -76.4 & -116.4 & -110.5 & -96.9 & -93.4 & -86.4 \\
\hline Gossypol-6,6'-dimethylether & -89.6 & -82.6 & -90.2 & -82.3 & -71.9 & -112.2 & -81.8 & -119.8 & -113.9 & -103.5 & -96.5 & -84.3 \\
\hline Gossypol-6-methylether & -82.2 & -90.0 & -87.5 & -82.4 & -69.4 & -113.0 & -75.3 & -115.2 & -111.7 & -103.6 & -101.0 & -83.4 \\
\hline Homalomenol C & -66.5 & -75.6 & -26.8 & -65.5 & -64.4 & -89.4 & -63.4 & -74.5 & -87.0 & -68.4 & -84.5 & -73.1 \\
\hline 1-Hydroperoxy-10(14),11-guaiadiene & -59.3 & -78.1 & -59.8 & -61.2 & -63.7 & -85.3 & -61.7 & -73.4 & -81.5 & -74.5 & -79.2 & -68.5 \\
\hline 10-Hydroperoxy-1,11-guaiadiene & -77.0 & -82.0 & -67.9 & -66.3 & -66.3 & -83.7 & -60.1 & -80.2 & -86.0 & -73.5 & -85.7 & -78.9 \\
\hline 14-Hydroperoxy-1(10),11-guaiadiene & -78.4 & -92.8 & -85.2 & -69.2 & -70.4 & -93.9 & -67.8 & -79.5 & -87.7 & -75.2 & -91.0 & -79.6 \\
\hline Kudtriol & -69.5 & -78.5 & -66.2 & -65.6 & -61.3 & -79.2 & -58.9 & -73.8 & -78.6 & -69.7 & -93.0 & -70.4 \\
\hline 5-epi-Kudtriol & -59.0 & -78.2 & -65.4 & -66.2 & -69.3 & -79.6 & -55.7 & -76.8 & -77.6 & -70.7 & -83.7 & -68.5 \\
\hline Longifolene & -52.4 & -69.7 & -35.7 & -56.3 & -57.6 & -74.0 & -52.4 & -63.5 & -68.1 & -63.1 & -66.8 & -60.8 \\
\hline Mukaadial & -65.9 & -86.9 & -61.9 & -72.3 & -72.0 & -92.6 & -58.7 & -77.1 & -83.7 & -74.5 & -91.5 & -69.6 \\
\hline Mustakone & -57.7 & -57.0 & -61.7 & -59.3 & -56.1 & -76.2 & -53.7 & -69.0 & -72.7 & -63.2 & -73.7 & -63.2 \\
\hline Muzigadial & -70.3 & -85.5 & -15.2 & -62.1 & -66.3 & -78.6 & -57.9 & -76.5 & -80.4 & -73.1 & -81.2 & -71.5 \\
\hline Nerolidol & -79.6 & -82.0 & -87.3 & -76.3 & -74.5 & -91.1 & -79.5 & -84.3 & -87.3 & -76.5 & -91.7 & -85.8 \\
\hline Oplopanone & -68.5 & -78.9 & -64.1 & -65.2 & -59.8 & -78.2 & -58.4 & -71.5 & -77.0 & -67.2 & -78.5 & -68.5 \\
\hline 10,12-Peroxycalamenene & -51.6 & -70.8 & -62.9 & -54.6 & -58.3 & -71.8 & -49.9 & -71.3 & -66.5 & -61.4 & -69.9 & -62.4 \\
\hline Plagiochilin A & -96.2 & -89.1 & -95.1 & -81.9 & -78.8 & -105.9 & -75.5 & -99.4 & -99.8 & -97.9 & -87.5 & -89.2 \\
\hline Polygodial & -60.0 & -80.5 & -57.6 & -68.6 & -62.1 & -81.9 & -55.2 & -73.3 & -78.4 & -69.2 & -84.7 & -76.3 \\
\hline Zingiberene $-3,6-\alpha-$ peroxide & -75.1 & -71.7 & -80.1 & -74.3 & -67.0 & -83.7 & -67.1 & -80.7 & -79.9 & -74.0 & -81.1 & -76.1 \\
\hline Zingiberene $-3,6-\beta-$ peroxide & -71.6 & -75.1 & -77.1 & -76.2 & -60.7 & -84.3 & -66.0 & -74.9 & -83.0 & -67.0 & -87.3 & -74.6 \\
\hline
\end{tabular}


Table 15. MolDock docking energies $(\mathrm{kJ} / \mathrm{mol})$ of miscellaneous sesquiterpenoids with Leishmania infantum protein targets.

\begin{tabular}{|c|c|c|c|c|c|}
\hline Miscellaneous Sesquiterpenoids & LinfCYP51 & LinfGLO2 & LinfPnC1 & LinfTDR1 & LinfTR \\
\hline Alloaromadendrene & -66.8 & -57.0 & -52.9 & -61.7 & -67.2 \\
\hline Aromadendrene & -60.8 & -59.7 & -60.8 & -61.6 & -76.8 \\
\hline 1,10-Bisaboladiene-3,4-diol & -75.6 & -70.8 & -63.5 & -72.1 & -85.0 \\
\hline$\alpha-$ Bisabolol & -76.5 & -74.1 & -73.4 & -75.0 & -74.7 \\
\hline Corymbolone & -65.9 & -54.6 & -49.1 & -59.4 & -65.3 \\
\hline $6 \alpha, 9 \alpha$-Dihydroxypolygodial & -75.7 & -62.3 & -23.0 & -63.6 & -78.0 \\
\hline$\alpha$-Eudesmol & -62.6 & -58.1 & -28.2 & -59.1 & -69.5 \\
\hline$\beta$-Eudesmol & -68.0 & -59.6 & -23.9 & -64.3 & -69.7 \\
\hline 1(10),5-Germacradien-4-ol & -70.4 & -58.4 & -52.6 & -63.6 & -70.2 \\
\hline Germacrene D & -64.6 & -57.4 & -62.7 & -68.0 & -70.2 \\
\hline Gossypol & -109.9 & -99.6 & no dock & -86.6 & -97.0 \\
\hline Gossypol-6,6'-dimethylether & -109.5 & -95.1 & no dock & -88.7 & -101.1 \\
\hline Gossypol-6-methylether & -113.1 & -99.6 & no dock & -94.5 & -100.9 \\
\hline Homalomenol C & -72.3 & -57.3 & -41.9 & -64.4 & -68.5 \\
\hline 1-Hydroperoxy-10(14),11-guaiadiene & -74.5 & -56.7 & -15.4 & -62.9 & -72.8 \\
\hline 10-Hydroperoxy-1,11-guaiadiene & -79.3 & -66.5 & -38.7 & -67.3 & -75.4 \\
\hline 14-Hydroperoxy-1(10),11-guaiadiene & -81.4 & -67.2 & -69.9 & -74.2 & -74.4 \\
\hline Kudtriol & -68.1 & -51.5 & no dock & -65.3 & -72.4 \\
\hline 5-epi-Kudtriol & -67.9 & -61.6 & no dock & -63.0 & -71.5 \\
\hline Longifolene & -64.9 & -49.4 & -48.0 & -54.6 & -61.5 \\
\hline Mukaadial & -74.9 & -61.5 & -21.0 & -63.7 & -78.0 \\
\hline Mustakone & -63.5 & -57.5 & -18.1 & -55.3 & -63.3 \\
\hline Muzigadial & -72.6 & -56.5 & -66.6 & -65.1 & -84.6 \\
\hline Nerolidol & -79.3 & -72.3 & -72.0 & -79.1 & -82.5 \\
\hline Oplopanone & -65.7 & -59.4 & -55.6 & -63.2 & -69.2 \\
\hline 10,12-Peroxycalamenene & -65.2 & -46.6 & no dock & -58.2 & -70.5 \\
\hline Plagiochilin A & -95.9 & -79.6 & no dock & -80.5 & -89.3 \\
\hline Polygodial & -72.1 & -57.6 & -45.3 & -62.8 & -72.3 \\
\hline Zingiberene $-3,6-\alpha-$ peroxide & -77.7 & -65.4 & -65.8 & -66.7 & -75.1 \\
\hline Zingiberene $-3,6-\beta$-peroxide & -69.8 & -65.5 & -51.2 & -62.7 & -72.9 \\
\hline
\end{tabular}

The guaianolide with the strongest docking energy was diguaiaperfolin, probably owing to its dimeric structure and larger molecular weight (716.77 amu). This ligand did show notable docking $(-154.5 \mathrm{~kJ} / \mathrm{mol})$ with LmajDHODH as well as with LmajUGPase (docking energy $=-151.8 \mathrm{~kJ} / \mathrm{mol}$ ). $8 \beta$-[4-Hydroxy-5-(5-hydroxytigloyloxy)tigloyl]santamarin was the strongest-docking eudesmanolide, and this ligand showed docking selectivity to LmajMetRS (docking energy $=-153.1 \mathrm{~kJ} / \mathrm{mol}$ ). The proteins most strongly targeted by both the guaianolides and the eudesmaolides were also LmajMetRS and LmajDHODH. Interestingly, the miscellaneous sesquiterpenoids preferentially targeted L. major pteridine reductase 1 (LmajPTR1), and plagiochilin A showed notable selectivity (docking energy $=$ $-132.2 \mathrm{~kJ} / \mathrm{mol}$ ) for this protein.

Several electrophilic sesquiterpenoids have exhibited antiprotozoal activity [5] and many of these showed docking selectivity to LmajDHODH. The active site of this protein has some potential 
nucleophilic residues, namely Ser 69, Ser 196, and Cys 131. Suitably oriented electrophilic ligands could form covalent bonds with these nucleophiles and thus inhibit the enzyme. Thus, for example, the germacranolide tatridin A docked preferentially to LmajDHODH, and the lowest-energy docked pose oriented the electrophilic carbon of the $\alpha$-methylene lactone moiety close to the sulfur atom of Cys 131 (see Figure 6). Similarly, the lowest-energy docked orientation of 11-epi-ivaxillin places one of the epoxide groups near to the sulfur atom of Cys 131 (Figure 7). Conversely, the lowest-energy docked pose of neurolenin B is such that the electrophilic carbon of the $\alpha$-methylene lactone group of the ligand is near the hydroxyl group of Ser 69 (Figure 8). The ligand with the lowest docking energy to LmajDHODH was the guaianolide dimer diguaiaperfolin $(-154.5 \mathrm{~kJ} / \mathrm{mol})$. The lowest-energy pose for this ligand placed the cyclopentenone moiety near the sulfur atom of Cys 131 (Figure 9).

Figure 6. Lowest-energy docked pose of tatridin A with L. major dihydroorotate dehydrogenase (LmajDHODH, PDB 3mhu). The cofactor, riboflavin monophosphate, is shown as a space-filling structure.

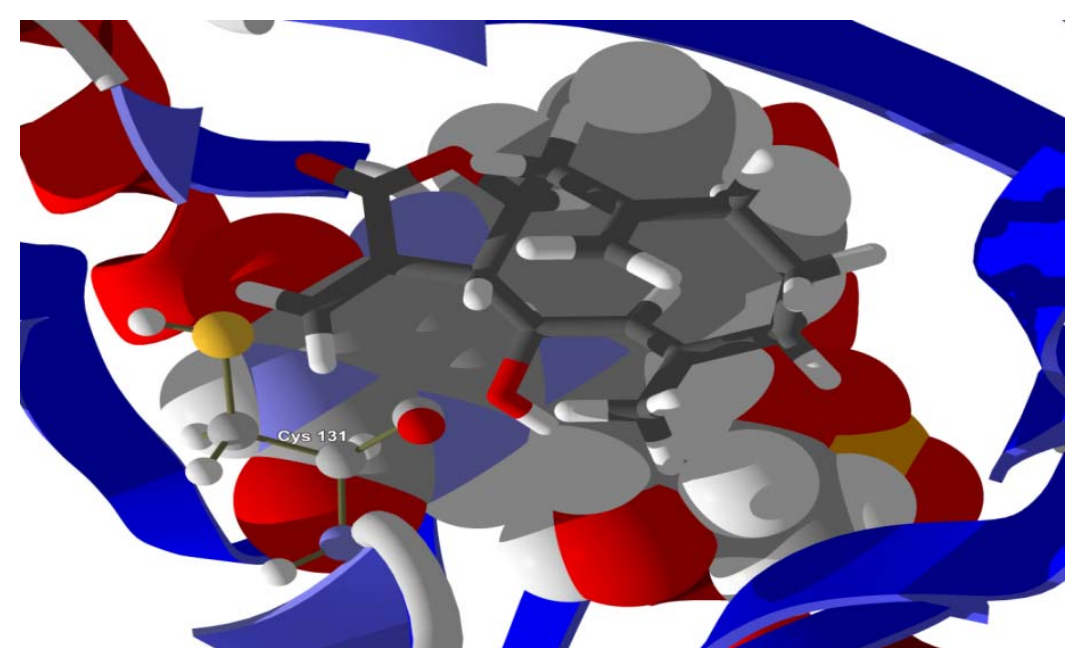

Figure 7. Lowest-energy docked pose of 11-epi-ivaxillin with L. major dihydroorotate dehydrogenase (LmajDHODH, PDB 3mhu). The cofactor, riboflavin monophosphate, is shown as a space-filling structure.

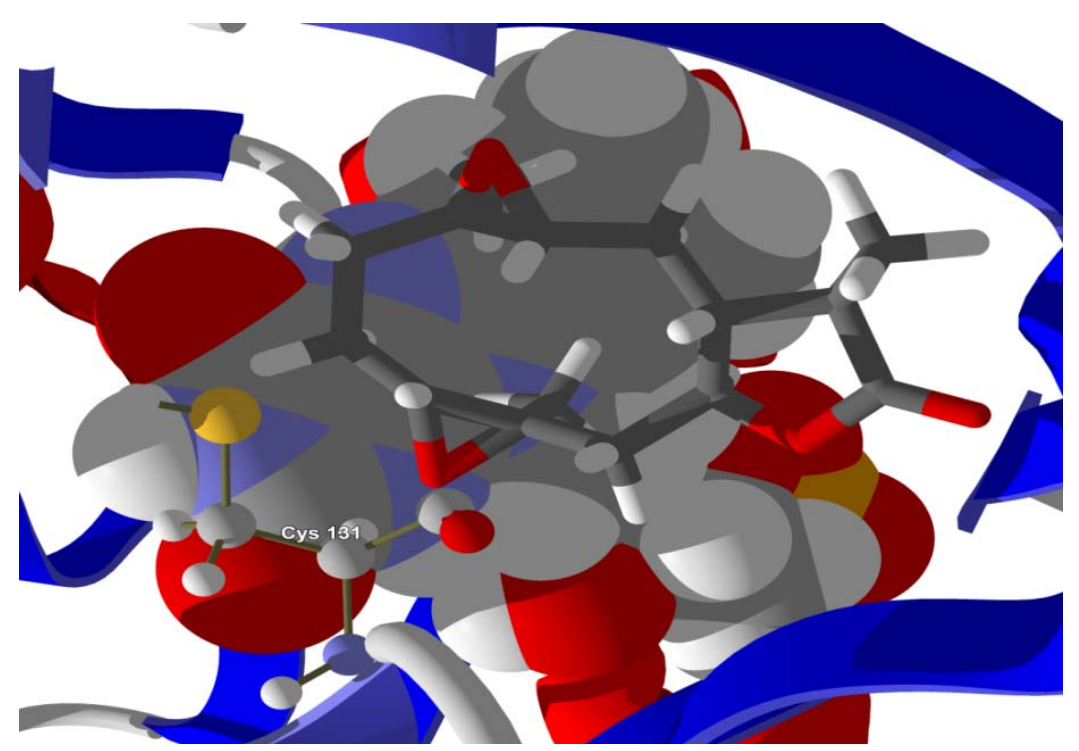


Figure 8. Lowest-energy docked pose of neurolenin B with L. major dihydroorotate dehydrogenase (LmajDHODH, PDB 3mhu). The cofactor, riboflavin monophosphate, is shown as a space-filling structure.

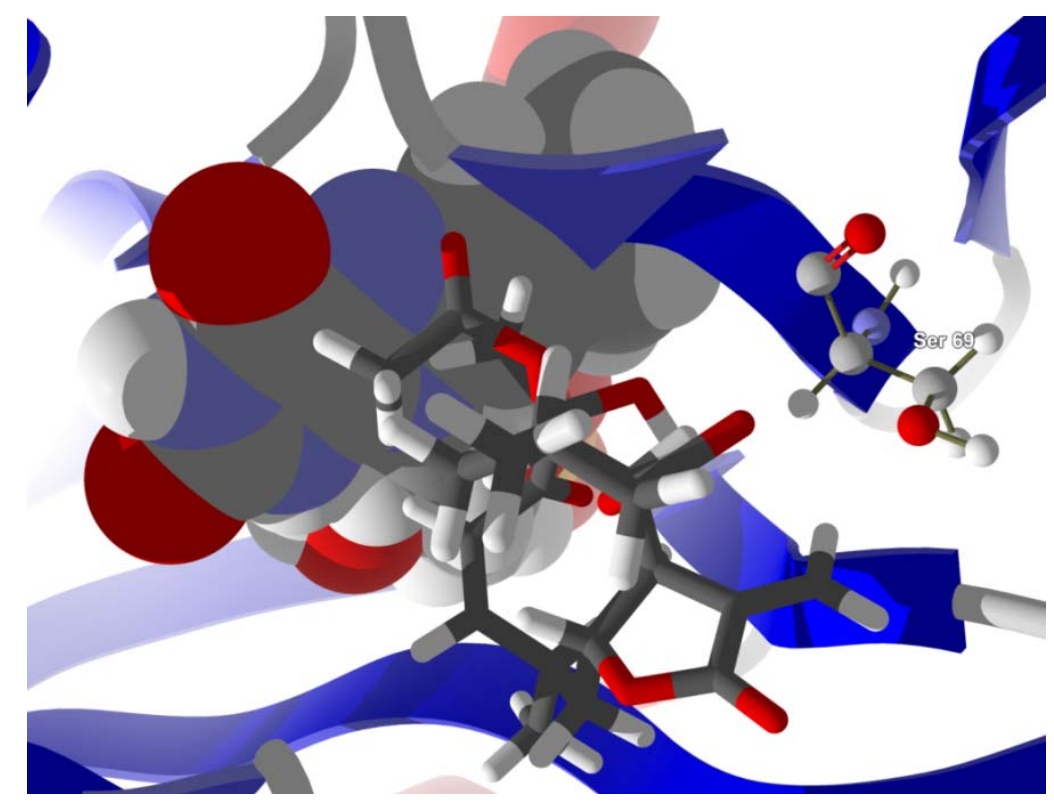

Figure 9. Lowest-energy docked pose of diguaiaperfolin with L. major dihydroorotate dehydrogenase (LmajDHODH, PDB 3mhu). The cofactor, riboflavin monophosphate, is shown as a space-filling structure.

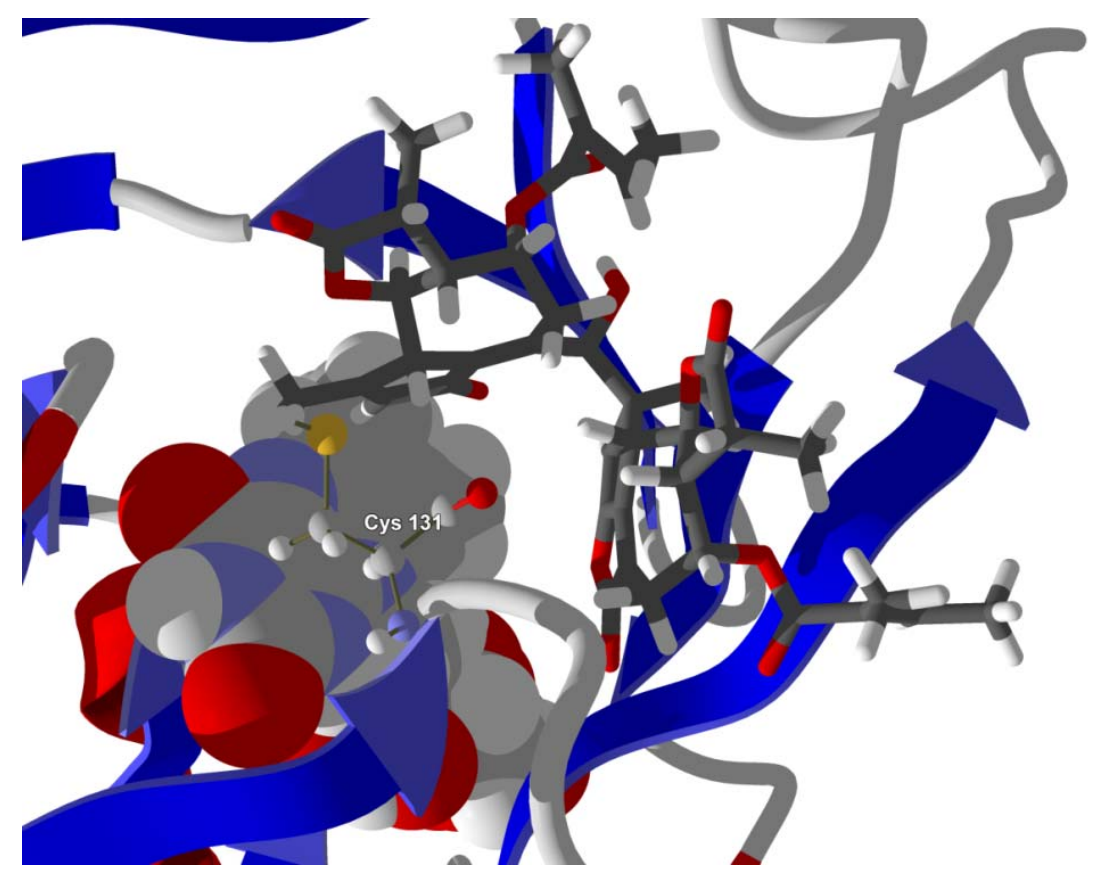

\subsection{Diterpenoid Docking}

Structures of diterpenoids are shown in Figures 10-18. Docking energies of the diterpenoids are assembled in Tables 16-28. The diterpenoids ligands generally favored docking to L. mexicana glycerol-3-phosphate dehydrogenase (LmexGPDH). In particular, the kaurane diterpenoids strongly docked to this target. In addition to LmexGPDH, labdane diterpenoids showed docking preferences for 
LmajMetRS and LmajDHODH. The strongest-docking ligands were the cinnamoyl cassanes $6 \beta-O$ cinnamoyl-12-hydroxy-(13)15-en-16,12-olide-18-cassaneoic acid and 6 $\beta$-O-2',3'-dihydro-cinnamoyl12-hydroxy-(13)15-en-16,12-olide-18-cassaneoic acid. These two ligands showed significant docking preference to LmajMetRS and LmexPMM.

Figure 10. Abietane diterpenoids examined in this study.

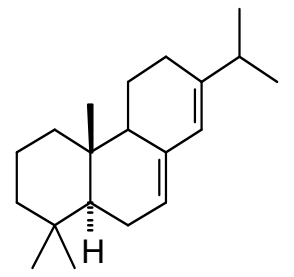

Abieta-7,13-diene<smiles>CC(C)C1=CC2=CC[C@H]3[C@@](C)(CO)CCC[C@]3(C)C2CC1</smiles>

4-epi-Abietol<smiles>CC(C)C1=CC(=O)C2=C(CC[C@H]3C(C)(C)CCC[C@@]23C)C1=O</smiles>

12-Deoxyroyleanone<smiles>COC1=C(C(C)C)C(=O)c2c(O)cc3c(c2C1=O)C1CC[C@@]3(C)O1</smiles>

Dracocequinone A<smiles>CC1c2c(O)cc3c(c2O[C@H]1O)CC[C@@H]1C(C)(C)CCCC31C</smiles>

ar-Abietatriene-12,16-diol14,16-oxide<smiles>CC1COC2=C1C(=O)C(=O)c1c2ccc2c1CCCC2(C)C</smiles><smiles>CC(C)c1ccc2c(c1)C(O)C[C@@H]1C(=O)[C@@]2(C)CCC[C@@H]1C(=O)O</smiles>

$9 \alpha, 13 \alpha$-epi-Dioxyabiet8(14)-en-18-oic acid<smiles>COC1=C(C(C)C)C(=O)c2c(O)cc3c(c2C1=O)C1CC[C@@]3(C)C(=O)O1</smiles>

Dracocequinone B<smiles>CC1COc2c3c(cc(O)c21)C1(C)CCCC(C)(C)[C@]1(C)C(=O)C3=O</smiles>

ar-Abietatrien-12-ol6,7-dione-14,16-oxide

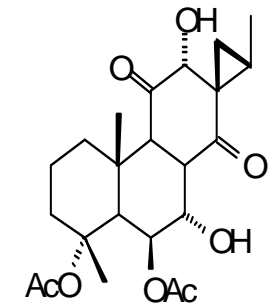

12-O-Deacetyl-6-Oacetyl-18acetyloxycoleon Q<smiles>CC(C)c1ccc2c(c1)C(O)C[C@@H]1C(=O)C2(C)CCC[C@@H]1CO</smiles>

$9 \alpha, 13 \alpha$-epi-Dioxyabiet8(14)en-18-ol<smiles>CC(C)c1cc2c(cc1O)[C@]1(C)CCCC(C)[C@H]1CC2</smiles>

Ferruginol<smiles>CC(C)C1=CC2=CC[C@H]3[C@@](C)(C(=O)O)CCC[C@]3(C)C2CC1</smiles>

epi-Abietic acid

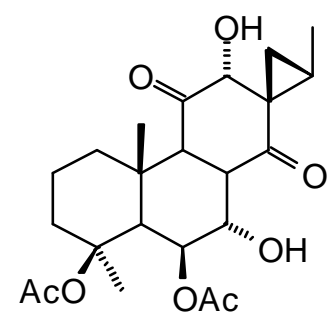

12-O-Deacetyl-6- $O$-acetyl-

19-acetyloxycoleon Q<smiles>COc1c(O)c2c(c(O)c1C(C)C)C(=O)CC1C23CCC[C@@]1(C)CO3</smiles>

Dracocephalone A<smiles>CC(C)c1cc2c(cc1O)[C@]1(C)CC[C@@H](O)C(C)(C)[C@@H]1CC2</smiles>

Hinokiol 
Figure 10. Cont.<smiles>CC(C)c1cc2c(cc1O)[C@]1(C)C(=O)C[C@@H](O)C(C)(C)[C@H]1CC2</smiles>

Hinokiol-1-one<smiles>CC(C)C1=CC2=CC[C@H]3C(C)(C)CCC[C@]3(C)C2=C(O)C1=O</smiles>

11-Hydroxy-7,9(11),13abietatrien-12-one<smiles></smiles>

14-Hydroxy-6oxoferruginol<smiles>CC(C)C1=Cc2ccc3c(c2C(=O)C1=O)C(=O)CCC3(C)C</smiles>
1-Oxomiltirone<smiles>CC(CO)c1cc2c(c(O)c1O)[C@@]1(C)CCCC(C)(C)C1=C(O)C2=O</smiles>

6,11,12,16-Tetrahydroxy5,8,11,13-abietatetraen-7one<smiles>CC(C)C1=CC2=C(C(=O)C1=O)[C@@]1(C)CCCC(C)(C)[C@@H]1CC2O</smiles>

7 $\beta$-Hydroxyabieta-8,13diene-11,12-dione<smiles>CC(C)C1=C(O)C(=O)C2=C(CC[C@H]3C(C)(C)C(=O)CC[C@@]23C)C1=O</smiles>

12-Hydroxy-8, 12abietadiene-3,11,14-trione<smiles>CC(C)c1cc2c(c(O)c1O)[C@@]1(C)CCCC(C)(C)C1=C(O)C2=O</smiles>
6-Hydroxysalvinolone<smiles>CC(C)C1=C(O)C(=O)C2=C(CC[C@@H]3C(C)CCC[C@@]23C)C1=O</smiles>

Royleanone<smiles>CC(C)c1c(O)cc2c(c1O)C(=O)C(O)=C1C(C)(C)CCC[C@@]12C</smiles>

6,12,14-Trihydroxyabieta5,8,11,13-tetraen-7-one<smiles>CC(C)c1ccc2c(c1)[C@H](O)C[C@H]1C(C)CCCC21C</smiles>

$7 \alpha$-Hydroxyabieta-

8,11,13-triene<smiles>CC1COC2=C1C(=O)C(=O)c1c2ccc2c1C(O)CCC2(C)C</smiles>

$1 \beta-$

Hydroxycryptotanshinone<smiles>COc1c(O)c2c(c(O)c1C(C)C)C(=O)C=C1C(C)(C)CCCC12C</smiles>

Komarovinone A<smiles>CC(C)c1cc2c(cc1O)C1(C)CCCC(C)(C)[C@H]1CC2=O</smiles>

Sugiol<smiles>CC(C)c1ccc2c(c1)CC[C@@H]1[C@@](C)(CO)[C@H](O)CC[C@@]21C</smiles>

4-epi-Triptobenzene L<smiles>CC(C)C1=C(O)C2=CC(=O)[C@]3(C)C(C)CCCC3(C)C2=CC1=O</smiles>

14-Hydroxy-7,9(11),13abietatriene-6,12-dione<smiles>COC1=C(C(C)C)C(=O)C2=C(O)C(=O)C3=C(C=CCC3(C)C)C2=C1</smiles>

7-Hydroxy-12-methoxy20-nor-abieta-

$1,5(10), 7,9,12$-pentaen6,14-dione<smiles>CC1COC2=C1C(=O)C(=O)c1c2ccc2c1C(=O)CCC2(C)C</smiles>

1-Oxocryptotanshinone<smiles>CC(C)C1=CC2=CC(=O)[C@]3(C)C(C)(C)CCC[C@]3(C)C2=C(O)C1=O</smiles>

Taxodione<smiles>CC1=C(C)C2=C(O)C(=O)c3c(O)c4c(c(O)c3[C@@]2(C)CC1)OC(C)C4</smiles>

Uncinatone 
Figure 11. Clerodane diterpenoids examined in this study.

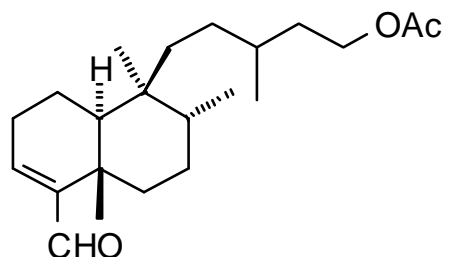

15-Acetoxy-cis-cleroden-3-en-18-al<smiles>CC(=O)OCCC(C)CC[C@]1(C)[C@@H](C)CC[C@]2(C)C(CO)=CCC[C@@H]21</smiles>

15-O-Acetylcistadiol<smiles>CC1=CC(=O)C[C@]2([C@@H]3CC(c4ccoc4)OC3=O)[C@@H](C)CC[C@H]12</smiles>

trans-Dehydrocrotonin

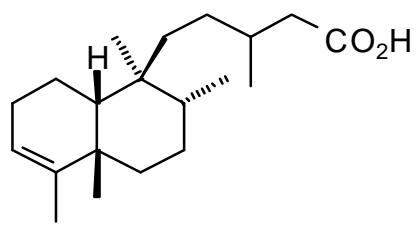

epi-Populifolic acid

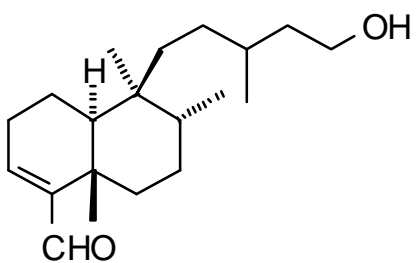

15-Hydroxy-cis-cleroden-3-en-18-al<smiles>C=C(CC(=O)O)C(=O)C[C@]1(C)[C@H](C)CC[C@]2(C)C(C)=CCC[C@@]21C</smiles>

ent-12-Oxo-3,13(16)-clerodien-15-oic acid

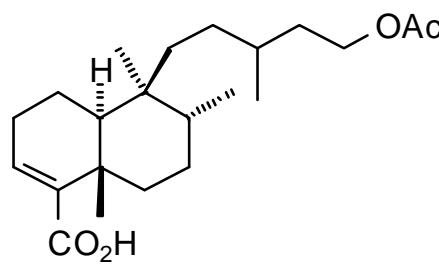

15-Acetoxy-cis-cleroden-3-en18-oic acid

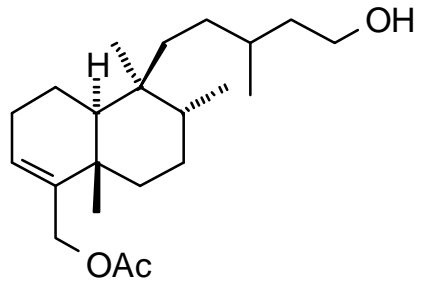

18-O-Acetylcistadiol

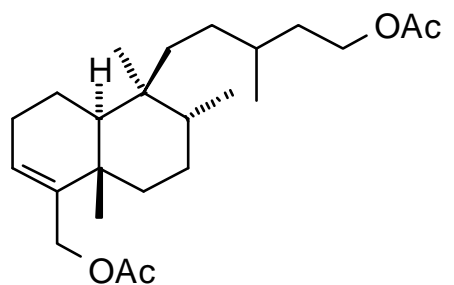

15,18-Di- $O$-acetylcistadiol

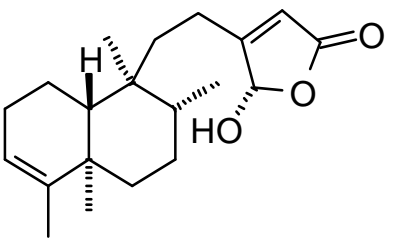

ent-16R-Hydroxy-3,13-

clerodadien-15,16-olide

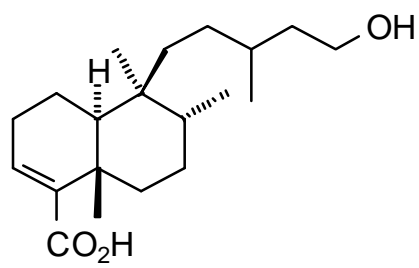

15-Hydroxy-cis-cleroden-3-en18-oic acid

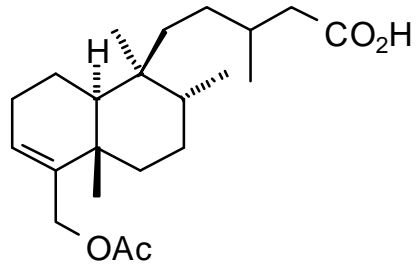

18-Acetoxy-cis-cleroden-3-en15-oic acid<smiles>CC(CCO)CC[C@]1(C)[C@H](C)CCC2(C)C(CO)=CCC[C@]21C</smiles>

Cistadiol<smiles>CC1=CCC[C@]2(C)[C@H](C)C(C)CC[C@]12C/C=C(\C)CCO</smiles>

8-epi-Kolavenol<smiles>CC1=CCC[C@]2(C)[C@H]1CC[C@H](C)[C@]21CCC2=CC(=O)OC2O1</smiles>

ent-16S-Hydroxy-3,13clerodadien-15,16-olide<smiles>CC(CC[C@]1(C)[C@@H](C)CCC2(C)C(CO)=CCC[C@@]21C)CC(=O)O</smiles>

18-Hydroxy-cis-cleroden-3-en15-oic acid 
Figure 12. Labdane diterpenoids examined in this study.<smiles>C=C[C@]1(C)CC[C@]2(C)[C@@H]3CC[C@H](OC(C)=O)C(C)(C)[C@@H]3CC[C@@]2(C)O1</smiles>

ent-3ß-Acetoxy-13-epimanoyl-oxide<smiles>C=C1CC[C@H]2C(C)(C)CCCC2(C)C1CC=C1C(OC)COC1OC</smiles>

Aulacocarpin D<smiles>C=C1CC[C@@H]2[C@H](C)CCCC2(C)C(CC/C(C)=C/C(C)=O)C1=C</smiles>

Dehydropinifolic acid 15-<smiles>C=C1CC[C@H]2C(C)(C)CCC[C@]2(C)C1C/C=C(/C=O)CC=O</smiles>

Labda-8(17),12-diene15,16-dial<smiles>C=C1CCC2(C)[C@@H](CO)[C@@H](O)CC[C@]2(C)[C@H]1CC=C1C(=O)OC[C@@H]1O</smiles>

Andrographolide<smiles>C=C/C(C)=C/CC1C(=C)CC[C@H]2[C@@H](C)CCCC12C</smiles>

trans-Communic acid $\mathrm{MeO}$<smiles>C=C1CC[C@H]2C(C)(C)CCCC2(C)C1CC(O)C1=C[C@@H](C)OC1=O</smiles>

12(S)-Hydroxy-15(R)methoxy-labdan-

8(17),13(14)-dien-15,16-<smiles>C=C/C(C)=C/CC1C(C)(C)[C@H](O)C[C@]2(C)C(C)CCCC12C</smiles>

Labda-12,14-dien-7 $\alpha, 8 \alpha-$ diol<smiles>CC1(C)CCCC2(C)C(C/C=C(\CO)[C@@H](O)CO)[C@]3(CC[C@@H]12)CO3</smiles>

14(R)-Aulacocarpin C<smiles>C=C/C(C)=C/CC1C(=C)CCC2(C)C(C)(OC(C)=O)CCCC12C</smiles>
trans-Communic acid methyl ester<smiles>C=C1CC[C@H]2C(C)(C)CCCC2(C)C1CC(O)C1=CC(OC)OC1=O</smiles>

12(S)-Hydroxy-15(S)methoxy-labdan8(17,)13(14)-dien-<smiles>CC(CCO)CCC1[C@@H]2CCC(C)(C)C(C)(CCCC1(C)C)C2(C)O</smiles><smiles>C=C1CC[C@H]2C(C)(C)C(O)CCC2(C)C1CCC(C)CCO</smilesLabdan-8 $\alpha, 15-$ diol
13(E)-Labda-7,13-dien-<smiles>CC1(C)CCCC2(C)[C@H](C/C=C(\CO)C(O)CO)[C@]3(CC[C@@]12C)CO3</smiles>

14(S)-Aulacocarpin C<smiles>C=C1CCC2(C)C(C)(C)CCC[C@]2(C)[C@H]1CC/C(C)=C/C(=O)O</smiles>

Copalic acid<smiles>C/C(=C\CO)CCC1C(C)(O)C=C[C@]2(C)C(C)(C)CCCC12C</smiles>

Labd-8(17)-en-3 $\beta, 15$-diol 
Figure 12. Cont.

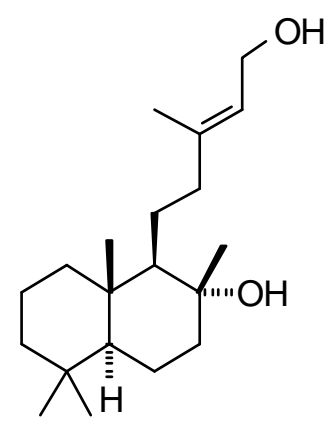

13(E)-Labd-13-en-8 $\alpha, 15$ -<smiles>C=C(CC(=O)O)C(=O)CC1=C(C)CC[C@H]2[C@@H](C)CCC[C@]12C</smiles>

ent-12-Oxo-8,13(16)-

labdadien-15-oic acid<smiles>C=C1CC[C@H]2C(C)(C)CCC[C@]2(C)[C@H]1/C=C/C1=C[C@H](OC)OC1=O</smiles>

15(R)-Methoxy-

labdan8(17),11(E),13(14)trien-15,16-olide<smiles>C=C1CC[C@H]2C(C)(C)CCC[C@]2(C)[C@H]1/C=C/C1=CC(OC)OC1=O</smiles>

15(S)-Methoxy-labdan8(17),11(E),13(14)-trien15,16-olide

Figure 13. Kaurane diterpenoids examined in this study.

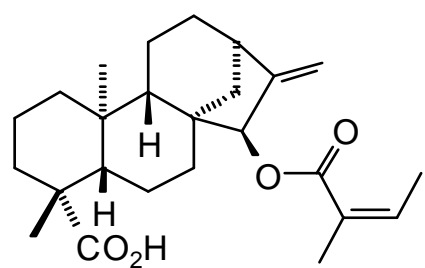

15-Angeloyl-4 $\alpha, 15 \beta$-kaur-16-en-18-oic acid

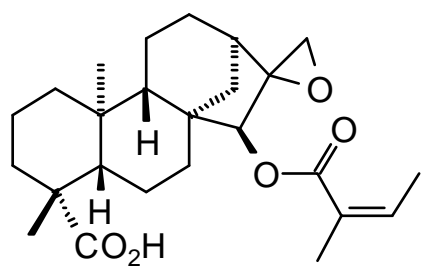

Perymenic acid<smiles>C=C1C(=O)[C@@H]2CC[C@H]3C(C)CCC[C@]3(C)[C@H]2C(O)C[C@H]1C</smiles>

ent-11 $\alpha$-Hydroxy-16-kauren-15-one

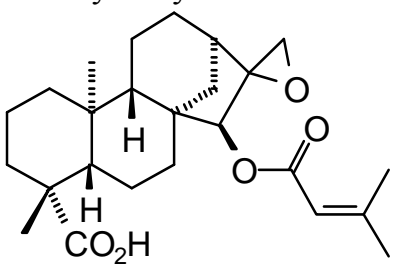

ent-15 $\beta$-Senecioyloxy-16,17-epoxykauran-18-oic acid

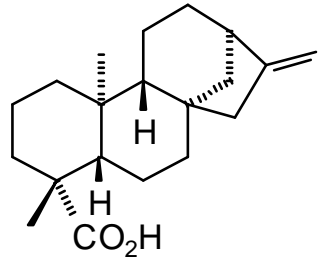

Kaurenoic acid 
Figure 14. Pimarane diterpenoids examined in this study.<smiles>C=C[C@]1(C)CC=C2C(CC[C@@H]3[C@@H](CO)CCC[C@@H]23)C1</smiles>

Acanthoic acid<smiles>C=C[C@]1(C)C=C2CC[C@H]3[C@](C)(CO)[C@@H](O)CC[C@]3(C)[C@H]2CC1</smiles>

ent-8(14),15-Pimaradien3ß,19-diol<smiles>C=C[C@]1(C)C=C2[C@@H](O)C[C@H]3[C@](C)(C(=O)O)CCC[C@]3(C)[C@H]2CC1</smiles>

73-Hydroxy-ent-pimara-

8(14),15-dien-19-oic acid<smiles>C=C[C@]1(C)C=C2CC[C@H]3[C@](C)(C(=O)O)CCC[C@]3(C)[C@H]2CC1</smiles>

ent-Pimara-8(14),15-dien19-oic acid

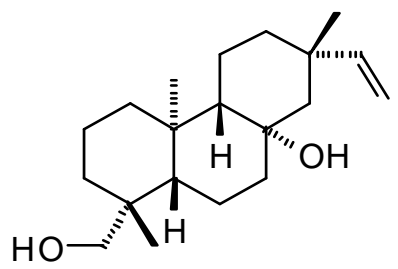

ent-15-Pimarene-8 8,19 -diol<smiles>C=C[C@]1(C)C=C2CC[C@H]3[C@@H](C)[C@@H](O)CC[C@]3(C)[C@H]2CC1</smiles>

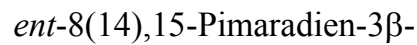

ol

Figure 15. Cassane diterpenoids examined in this study.

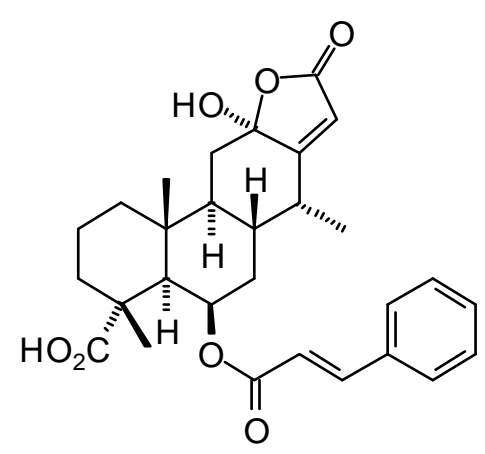

6ß-O-Cinnamoyl-12-hydroxy-(13)15en-16,12-olide-18-cassaneoic acid<smiles>CC(=O)OC1Cc2occc2[C@@H](C)C2C(C)(CC3(C)CCCC(C)(C)C23C)C1OC(C)=O</smiles>

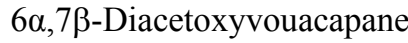<smiles>C=C[C@]1(C)C=C2CC[C@]3(C)[C@@H](C)[C@@H](OC(C)=O)CC[C@]3(C)[C@H]2CC1</smiles>

ent-8(14),15-

Pimaradien-3 $\beta$-acetoxy

Figure 16. Icetaxane diterpenoids examined in this study.<smiles></smiles>

Cyclocoulterone

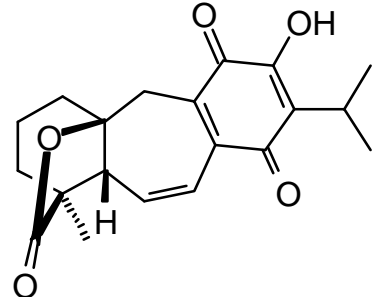

5-epi-Icetexone

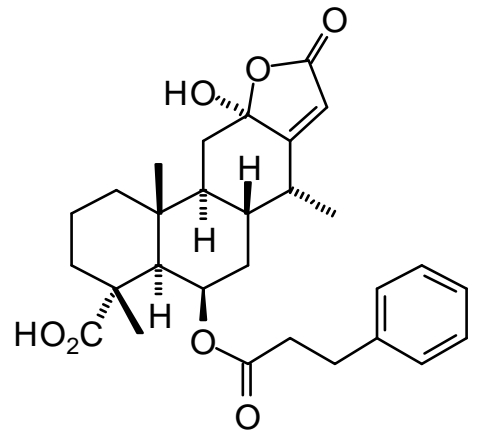

$6 \beta-O-2$ '3'-Dihydrocinnamoyl-12hydroxy-(13)15-en-16,12-olide-18cassaneoic acid 
Figure 17. Mulinane diterpenoids examined in this study.

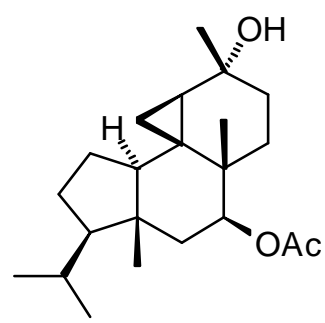

Azorellanol

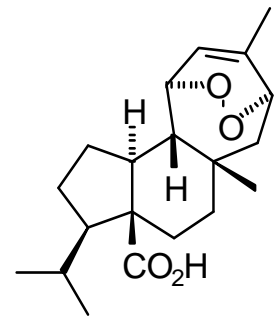

Mulinic acid

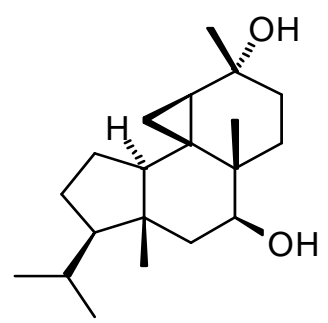

7-Deacetylazorellanol

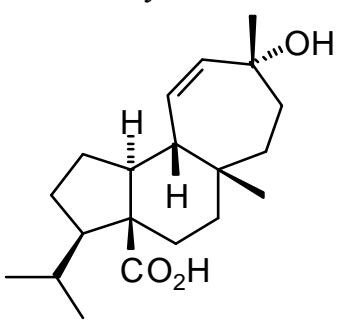

Mulinolic acid

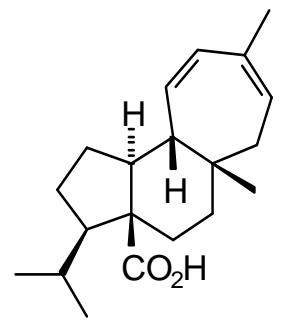

Mulin-11,13-dien-20-oic acid

Figure 18. Miscellaneoous diterpenoids examined in this study.

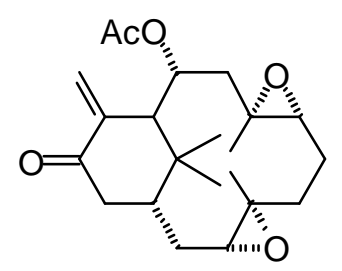

Hypoestoxide

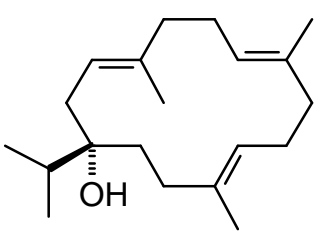

Serratol<smiles>COC1=C(C(C)C)C(O)=C2C(=O)OC34CCCC(C)(C)[C@H]3CC24CC1=O</smiles>

Komarovispirone<smiles>CC(C)c1c(O)ccc2c1CC[C@H]1C(C)(C)CCCC21C</smiles>

Totarol<smiles>CC(C)=CCC[C@]1(C)CCCC2(C)C(C=O)C(C=O)=CCC21</smiles>

Sacculatal 
Table 16. MolDock docking energies $(\mathrm{kJ} / \mathrm{mol})$ of abietane diterpenoids with Leishmania major protein targets.

\begin{tabular}{|c|c|c|c|c|c|c|c|c|c|c|c|c|}
\hline Abietane diterpenoids & LmajCatB & LmajDHODH & LmajdUTPase & LmajNDKb & LmajNH & LdonNMT & LmajOPB & LmajPDE1 & LmajPTR1 & LmajMetRS & LmajTyrRS & LmajUGPase \\
\hline Abieta-7,13-diene & -71.5 & -80.8 & -79.3 & -80.3 & -76.3 & -69.7 & -82.2 & -77.0 & -74.7 & -96.9 & -78.1 & -78.3 \\
\hline $\begin{array}{l}\text { ar-Abietatriene-12,16-diol-14,16- } \\
\text { oxide }\end{array}$ & -74.0 & -97.8 & -78.9 & -96.2 & -78.0 & -74.8 & -98.2 & -83.2 & -73.8 & -89.0 & -84.6 & -77.8 \\
\hline $\begin{array}{l}\text { ar-Abietatrien-12-ol-6,7-dione- } \\
\text { 14,16-oxide }\end{array}$ & -66.1 & -91.8 & -86.1 & -79.4 & -72.8 & -74.2 & -98.2 & -83.6 & -84.5 & -92.7 & -91.4 & -83.4 \\
\hline epi-Abietic acid & -75.3 & -93.5 & -81.8 & -74.9 & -84.0 & -78.1 & -86.1 & -80.7 & -82.1 & -99.8 & -87.3 & -84.7 \\
\hline 4-epi-Abietol & -71.9 & -87.6 & -81.8 & -80.4 & -80.9 & -69.3 & -83.4 & -74.8 & -78.1 & -96.3 & -81.0 & -82.3 \\
\hline Cryptotanshinone & -69.2 & -93.7 & -74.6 & -81.3 & -59.6 & -78.3 & -91.6 & -83.4 & -81.2 & -105.2 & -86.5 & -78.6 \\
\hline $\begin{array}{l}12-O-\text { Deacetyl-6-O-acetyl-18- } \\
\text { acetyloxycoleon Q }\end{array}$ & -81.2 & -89.0 & -95.0 & -89.7 & -99.9 & -79.5 & -95.5 & -111.8 & -93.9 & -94.2 & -88.9 & -114.2 \\
\hline $\begin{array}{l}12-O-\text { Deacetyl-6- }-O^{-} \text {acetyl-19- } \\
\text { acetyloxycoleon } \mathrm{Q}\end{array}$ & -76.3 & -104.3 & -82.9 & -93.8 & -101.2 & -75.4 & -100.7 & -107.4 & -84.3 & -99.0 & -92.0 & -96.9 \\
\hline 12-Deoxyroyleanone & -78.2 & -79.2 & -74.3 & -74.8 & -72.9 & -73.1 & -76.5 & -83.6 & -86.4 & -85.9 & -77.6 & -77.4 \\
\hline $\begin{array}{l}9 \alpha, 13 \alpha-e p i-\text { Dioxyabiet-8(14)-en- } \\
18-\text { oic acid }\end{array}$ & -65.5 & -88.2 & -79.9 & -82.1 & -80.1 & -64.0 & -89.6 & -79.4 & -71.2 & -89.5 & -88.2 & -81.6 \\
\hline $\begin{array}{l}9 \alpha, 13 \alpha-e p i-\text { Dioxyabiet-8(14)en- } \\
18-\text { ol }\end{array}$ & -47.3 & -86.6 & -76.2 & -78.7 & -72.3 & -65.3 & -74.4 & -76.8 & -72.7 & -81.5 & -74.0 & -71.2 \\
\hline Dracocephalone A & -66.5 & -82.6 & -70.8 & -76.6 & -71.9 & -66.7 & -93.0 & -83.2 & -87.4 & -83.4 & -72.9 & -78.7 \\
\hline Dracocequinone A & -66.6 & -84.4 & -74.5 & -76.0 & -74.1 & -75.4 & -94.8 & -83.5 & -73.8 & -87.6 & -76.4 & -82.6 \\
\hline Dracocequinone B & -74.5 & -89.1 & -71.5 & -78.4 & -78.4 & -76.6 & -83.4 & -81.9 & -76.0 & -88.7 & -77.3 & -88.2 \\
\hline Ferruginol & -68.7 & -90.3 & -81.0 & -81.7 & -76.0 & -71.3 & -85.3 & -82.2 & -83.7 & -99.3 & -83.4 & -81.2 \\
\hline Hinokiol & -77.9 & -88.2 & -85.2 & -75.8 & -79.1 & -62.3 & -84.5 & -74.9 & -77.9 & -92.4 & -77.7 & -74.0 \\
\hline Hinokiol-1-one & -79.9 & -87.2 & -87.6 & -80.6 & -81.8 & -70.4 & -86.5 & -76.7 & -81.6 & -97.4 & -82.2 & -77.5 \\
\hline $\begin{array}{l}7 \beta-\text { Hydroxyabieta- } 8,13-\text { diene- } \\
\text { 11,12-dione }\end{array}$ & -76.2 & -77.8 & -94.0 & -83.9 & -79.9 & -76.5 & -91.4 & -84.1 & -81.3 & -95.2 & -86.2 & -83.9 \\
\hline $7 \alpha-$ Hydroxyabieta $-8,11,13$-triene & -76.8 & -85.3 & -84.5 & -75.4 & -69.1 & -66.7 & -83.0 & -81.3 & -77.8 & -90.9 & -78.7 & -82.0 \\
\hline
\end{tabular}


Table 16. Cont.

\begin{tabular}{|c|c|c|c|c|c|c|c|c|c|c|c|c|}
\hline Abietane diterpenoids & LmajCatB & LmajDHODH & LmajdUTPase & LmajNDKb & LmajNH & LdonNMT & LmajOPB & LmajPDE1 & LmajPTR1 & LmajMetRS & LmajTyrRS & LmajUGPase \\
\hline $\begin{array}{l}\text { 14-Hydroxy-7,9(11),13-abietatriene- } \\
6,12-\text { dione }\end{array}$ & -74.8 & -83.5 & -71.0 & -82.1 & -68.6 & -76.5 & -88.3 & -76.2 & -78.6 & -94.8 & -84.7 & -85.5 \\
\hline $\begin{array}{l}\text { 11-Hydroxy-7,9(11),13-abietatrien- } \\
12-\text { one }\end{array}$ & -72.5 & -82.5 & -80.1 & -82.3 & -75.3 & -72.1 & -83.7 & -73.6 & -82.4 & -95.9 & -74.1 & -80.4 \\
\hline $\begin{array}{l}\text { 12-Hydroxy-8,12-abietadiene- } \\
3,11,14 \text {-trione }\end{array}$ & -75.9 & -86.0 & -75.2 & -79.9 & -83.7 & -73.9 & -90.3 & -84.7 & -94.2 & -85.3 & -79.6 & -86.0 \\
\hline $1 \beta$-Hydroxycryptotanshinone & -74.7 & -96.0 & -70.0 & -80.8 & -70.1 & -79.2 & -99.1 & -83.8 & -84.0 & -104.4 & -90.0 & -78.5 \\
\hline 7-Hydroxy-12-methoxy-20-nor- & & & & & & & & & & & & \\
\hline $\begin{array}{l}\text { abieta-1,5(10),7,9,12-pentaen- } 6,14- \\
\text { dione }\end{array}$ & -66.6 & -91.6 & -75.4 & -80.9 & -72.5 & -79.2 & -92.5 & -82.4 & -83.6 & -94.2 & -83.7 & -81.5 \\
\hline 14-Hydroxy-6-oxoferruginol & -56.1 & -91.1 & -72.3 & -85.5 & -75.7 & -73.9 & -87.0 & -79.5 & -83.6 & -90.3 & -75.2 & -79.3 \\
\hline 6-Hydroxysalvinolone & -77.1 & -83.3 & -76.6 & -76.2 & -77.6 & -72.4 & -82.3 & -81.2 & -82.7 & -93.7 & -87.1 & -86.5 \\
\hline Komarovinone A & -82.0 & -82.4 & -79.2 & -73.1 & -72.7 & -67.1 & -96.1 & -83.5 & -84.6 & -100.0 & -77.6 & -84.0 \\
\hline 1-Oxocryptotanshinone & -75.8 & -102.9 & -74.7 & -77.9 & -70.6 & -75.4 & -96.6 & -79.8 & -82.4 & -114.6 & -89.3 & -78.0 \\
\hline 1-Oxomiltirone & -71.6 & -84.7 & -78.4 & -77.8 & -71.6 & -76.8 & -90.3 & -82.8 & -79.8 & -104.9 & -82.4 & -79.1 \\
\hline Royleanone & -73.5 & -84.0 & -72.2 & -81.9 & -79.6 & -72.3 & -90.5 & -82.7 & -91.0 & -87.9 & -80.5 & -83.9 \\
\hline Sugiol & -67.6 & -90.5 & -82.4 & -73.2 & -81.5 & -53.1 & -76.2 & -84.0 & -77.2 & -97.3 & -84.0 & -84.4 \\
\hline Taxodione & -79.0 & -87.2 & -77.8 & -83.5 & -75.7 & -72.3 & -85.6 & -78.3 & -69.0 & -90.7 & -82.0 & -80.4 \\
\hline $\begin{array}{l}6,11,12,16-\text { Tetrahydroxy }-5,8,11,13- \\
\text { abietatetraen-7-one }\end{array}$ & -78.2 & -88.6 & -81.5 & -82.9 & -80.2 & -73.3 & -95.1 & -87.8 & -88.3 & -93.8 & -83.4 & -90.7 \\
\hline $\begin{array}{l}6,12,14-\text { Trihydroxyabieta }-5,8,11,13- \\
\text { tetraen-7-one }\end{array}$ & -69.6 & -86.1 & -70.6 & -78.7 & -73.9 & -80.8 & -89.4 & -84.6 & -81.5 & -97.4 & -85.9 & -83.5 \\
\hline 4-epi-Triptobenzene L & -68.9 & -94.9 & -89.9 & -81.7 & -79.6 & -76.6 & -87.6 & -85.9 & -83.3 & -88.2 & -82.4 & -82.4 \\
\hline Uncinatone & -84.3 & -81.8 & -81.6 & -85.9 & -62.1 & -74.5 & -86.5 & -86.5 & -108.8 & -107.7 & -95.6 & -77.6 \\
\hline
\end{tabular}


Table 17. MolDock docking energies (kJ/mol) of abietane diterpenoids with Leishmania donovani and L. mexicana protein targets.

\begin{tabular}{|c|c|c|c|c|c|c|c|c|c|c|c|c|}
\hline Abietane diterpenoids & LdonCatB & LdonCyp & LdonDHODH & LdonNMT & LmexGAPDH & LmexGPDH & LmexPGI & LmexPMM & $\begin{array}{l}\text { LmexPYK } \\
\text { Site } 1 \\
\end{array}$ & $\begin{array}{c}\text { LmexPYK } \\
\text { Site } 2 \\
\end{array}$ & $\begin{array}{c}\text { LmexPYK } \\
\text { Site } 3 \\
\end{array}$ & LmexTIM \\
\hline Abieta-7,13-diene & -73.0 & -84.2 & -70.8 & -69.7 & -69.1 & -92.6 & -62.7 & -76.6 & -81.3 & -71.3 & -74.2 & -62.9 \\
\hline ar-Abietatriene-12,16-diol-14,16-oxide & -77.9 & -84.2 & -70.5 & -74.8 & -73.7 & -104.7 & -74.6 & -82.6 & -104.4 & -83.5 & -84.9 & -73.1 \\
\hline $\begin{array}{l}\text { ar-Abietatrien-12-ol-6,7-dione-14,16- } \\
\text { oxide }\end{array}$ & -76.3 & -90.1 & -75.9 & -74.2 & -69.1 & -102.3 & -68.9 & -93.5 & -99.2 & -87.3 & -90.1 & -72.1 \\
\hline epi-Abietic acid & -77.4 & -86.4 & -67.6 & -78.1 & -60.9 & -97.6 & -67.8 & -81.5 & -84.0 & -74.5 & -91.0 & -68.6 \\
\hline 4-epi-Abietol & -77.2 & -88.0 & -69.7 & -69.3 & -68.1 & -94.0 & -66.3 & -78.8 & -78.0 & -75.2 & -72.6 & -65.9 \\
\hline Cryptotanshinone & -77.5 & -80.5 & -84.5 & -78.3 & -66.6 & -101.9 & -71.1 & -84.4 & -95.4 & -75.3 & -71.7 & -65.3 \\
\hline $\begin{array}{l}12-O-\text { Deacetyl-6-O-acetyl-18- } \\
\text { acetyloxycoleon } \mathrm{Q}\end{array}$ & -78.6 & -93.4 & -41.3 & -79.5 & -86.6 & -108.4 & -87.4 & -109.1 & -102.6 & -110.1 & -102.8 & -86.7 \\
\hline $\begin{array}{l}12-O-\text { Deacetyl-6-O-acetyl-19- } \\
\text { acetyloxycoleon } \mathrm{Q}\end{array}$ & -99.6 & -80.4 & -45.8 & -75.4 & -90.6 & -105.1 & -87.4 & -109.7 & -101.1 & -96.2 & -88.7 & -73.6 \\
\hline 12-Deoxyroyleanone & -80.4 & -86.7 & -72.1 & -73.1 & -68.2 & -99.6 & -62.7 & -82.7 & -84.0 & -73.0 & -87.4 & -68.4 \\
\hline $\begin{array}{l}9 \alpha, 13 \alpha-e p i-\text { Dioxyabiet }-8(14)-\text { en-18- } \\
\text { oic acid }\end{array}$ & -59.0 & -88.1 & -51.5 & -64.0 & -71.2 & -100.3 & -69.3 & -86.9 & -82.4 & -77.1 & -85.3 & -76.1 \\
\hline $9 \alpha, 13 \alpha-e p i-$ Dioxyabiet-8(14)en-18-ol & -55.8 & -76.8 & -58.2 & -65.3 & -63.2 & -96.6 & -66.9 & -81.2 & -79.7 & -71.6 & -74.1 & -70.9 \\
\hline Dracocephalone A & -74.3 & -81.2 & -73.4 & -66.7 & -69.5 & -103.5 & -64.4 & -82.2 & -84.9 & -74.8 & -81.2 & -68.1 \\
\hline Dracocequinone $\mathrm{A}$ & -71.2 & -83.6 & -77.7 & -75.4 & -66.1 & -104.7 & -66.2 & -82.5 & -84.7 & -72.0 & -79.9 & -68.9 \\
\hline Dracocequinone B & -75.7 & -81.6 & -72.0 & -76.6 & -67.4 & -107.2 & -67.7 & -83.7 & -82.8 & -72.2 & -77.3 & -76.7 \\
\hline Ferruginol & -70.5 & -91.1 & -75.4 & -71.3 & -71.2 & -96.7 & -75.1 & -83.7 & -82.9 & -75.3 & -76.1 & -69.8 \\
\hline Hinokiol & -77.1 & -87.8 & -72.0 & -62.3 & -71.1 & -95.9 & -65.9 & -79.3 & -81.4 & -71.9 & -73.5 & -63.7 \\
\hline Hinokiol-1-one & -77.0 & -86.1 & -72.7 & -70.4 & -70.9 & -99.6 & -63.7 & -80.2 & -79.7 & -69.7 & -77.2 & -67.6 \\
\hline $\begin{array}{l}7 \beta-\text { Hydroxyabieta- } 8,13-\text { diene- } 11,12- \\
\text { dione }\end{array}$ & -83.8 & -79.8 & -80.7 & -76.5 & -69.0 & -100.8 & -69.4 & -89.6 & -91.6 & -77.9 & -83.9 & -76.8 \\
\hline $7 \alpha-$ Hydroxyabieta-8,11,13-triene & -75.4 & -76.3 & -73.1 & -66.7 & -68.1 & -96.5 & -70.5 & -78.2 & -82.5 & -74.8 & -82.9 & -67.0 \\
\hline $\begin{array}{l}\text { 14-Hydroxy-7,9(11),13-abietatriene- } \\
6,12 \text {-dione }\end{array}$ & -74.5 & -89.5 & -81.6 & -76.5 & -72.7 & -96.0 & -68.6 & -86.8 & -85.3 & -76.6 & -75.3 & -67.9 \\
\hline
\end{tabular}


Table 17. Cont.

\begin{tabular}{|c|c|c|c|c|c|c|c|c|c|c|c|c|}
\hline Abietane diterpenoids & LdonCatB & LdonCyp & LdonDHODH & LdonNMT & LmexGAPDH & LmexGPDH & LmexPGI & LmexPMM & $\begin{array}{c}\text { LmexPYK } \\
\text { Site } 1 \\
\end{array}$ & $\begin{array}{c}\text { LmexPYK } \\
\text { Site } 2 \\
\end{array}$ & $\begin{array}{c}\text { LmexPYK } \\
\text { Site } 3 \\
\end{array}$ & LmexTIM \\
\hline $\begin{array}{l}\text { 11-Hydroxy-7,9(11),13-abietatrien- } \\
\text { 12-one }\end{array}$ & -79.6 & -87.1 & -76.1 & -72.1 & -61.8 & -97.7 & -68.7 & -83.9 & -78.1 & -76.3 & -78.1 & -69.9 \\
\hline $\begin{array}{l}\text { 12-Hydroxy-8,12-abietadiene- } \\
\text { 3,11,14-trione }\end{array}$ & -79.7 & -82.0 & -75.6 & -73.9 & -66.2 & -109.4 & -68.9 & -92.5 & -88.8 & -75.3 & -84.8 & -71.0 \\
\hline 1b-Hydroxycryptotanshinone & -78.4 & -84.0 & -80.5 & -79.2 & -68.3 & -98.5 & -71.1 & -87.5 & -94.5 & -77.8 & -74.5 & -70.8 \\
\hline $\begin{array}{l}\text { 7-hydroxy-12-methoxy-20-nor-abiet } \\
\text { a-1,5(10),7,9,12-pentaen-6,14-dione }\end{array}$ & -75.6 & -87.7 & -69.1 & -79.2 & -67.5 & -102.0 & -68.2 & -87.1 & -88.6 & -68.1 & -79.9 & -65.2 \\
\hline 14-Hydroxy-6-oxoferruginol & -66.5 & -93.7 & -78.2 & -73.9 & -71.7 & -96.9 & -61.8 & -87.2 & -88.7 & -80.8 & -70.6 & -68.5 \\
\hline 6-Hydroxysalvinolone & -78.8 & -78.9 & -77.6 & -72.4 & -62.2 & -94.7 & -66.0 & -89.9 & -94.9 & -84.3 & -75.8 & -69.0 \\
\hline Komarovinone A & -80.3 & -81.2 & -81.2 & -67.1 & -65.0 & -98.3 & -69.2 & -90.1 & -86.5 & -80.4 & -70.8 & -73.4 \\
\hline 1-Oxocryptotanshinone & -76.1 & -80.4 & -71.9 & -75.4 & -66.9 & -95.0 & -69.1 & -85.7 & -89.6 & -79.8 & -77.1 & -65.4 \\
\hline 1-Oxomiltirone & -74.3 & -71.4 & -78.1 & -76.8 & -61.6 & -92.9 & -65.3 & -81.5 & -84.5 & -76.6 & -71.2 & -66.5 \\
\hline Royleanone & -80.1 & -85.8 & -75.6 & -72.3 & -61.3 & -102.2 & -65.3 & -87.9 & -90.8 & -77.4 & -73.0 & -69.1 \\
\hline Sugiol & -73.9 & -78.4 & -77.3 & -53.1 & -65.1 & -95.8 & -66.2 & -85.3 & -86.3 & -79.1 & -71.1 & -71.8 \\
\hline Taxodione & -80.6 & -94.1 & -77.9 & -72.3 & -68.0 & -97.3 & -70.5 & -89.2 & -81.6 & -79.0 & -75.8 & -49.5 \\
\hline $\begin{array}{l}6,11,12,16-\text { Tetrahydroxy- } 5,8,11,13- \\
\text { abietatetra-en-7-one }\end{array}$ & -79.0 & -86.0 & -84.9 & -73.3 & -66.9 & -96.1 & -65.9 & -95.6 & -105.1 & -87.5 & -77.6 & -75.5 \\
\hline $\begin{array}{l}6,12,14-\text { Trihydroxyabieta }-5,8,11,13- \\
\text { tetraen-7-one }\end{array}$ & -72.7 & -84.9 & -81.2 & -80.8 & -72.6 & -97.7 & -62.5 & -89.1 & -89.8 & -89.2 & -77.1 & -62.8 \\
\hline 4-epi-Triptobenzene L & -76.3 & -89.7 & -76.3 & -76.6 & -72.3 & -101.5 & -70.0 & -85.1 & -85.7 & -77.6 & -77.9 & -72.5 \\
\hline Uncinatone & -85.4 & -73.3 & -72.1 & -74.5 & -69.8 & -89.0 & -65.3 & -84.4 & -94.8 & -84.1 & -89.2 & -64.4 \\
\hline
\end{tabular}


Table 18. MolDock docking energies $(\mathrm{kJ} / \mathrm{mol})$ of abietane diterpenoids with Leishmania infantum protein targets.

\begin{tabular}{|c|c|c|c|c|c|}
\hline Abietane diterpenoids & LinfCYP51 & LinfGLO2 & LinfPnC1 & LinfTDR1 & LinfTR \\
\hline Abieta-7,13-diene & -76.4 & -71.1 & no dock & -65.7 & -72.6 \\
\hline ar-Abietatriene-12,16-diol-14,16-oxide & -87.3 & -76.4 & no dock & -74.3 & -78.4 \\
\hline $\begin{array}{l}\text { ar-Abietatrien-12-ol-6,7-dione-14,16- } \\
\text { oxide }\end{array}$ & -84.7 & -72.7 & no dock & -76.8 & -83.5 \\
\hline epi-Abietic acid & -83.8 & -67.7 & no dock & -66.4 & -74.5 \\
\hline 4-epi-Abietol & -78.5 & -68.9 & no dock & -64.6 & -72.4 \\
\hline Cryptotanshinone & -83.6 & -67.0 & no dock & -71.5 & -84.1 \\
\hline $\begin{array}{l}12-O-\text { Deacetyl- } 6-O-\text { acetyl }-18- \\
\text { acetyloxycoleon } \mathrm{Q}\end{array}$ & -106.3 & -80.7 & no dock & -88.6 & -103.4 \\
\hline $\begin{array}{l}12-O-\text { Deacetyl }-6-O-\text { acetyl }-19- \\
\text { acetyloxycoleon } \mathrm{Q}\end{array}$ & -105.1 & -85.8 & no dock & -87.3 & -98.2 \\
\hline 12-Deoxyroyleanone & -81.7 & -72.8 & no dock & -67.2 & -77.2 \\
\hline $\begin{array}{l}9 \alpha, 13 \alpha-e p i-\text { Dioxyabiet}-8(14)-\text { en-18-oic } \\
\text { acid }\end{array}$ & -90.4 & -73.1 & no dock & -68.7 & -77.1 \\
\hline $9 \alpha, 13 \alpha-e p i-$ Dioxyabiet$-8(14)$ en-18-ol & -80.3 & -70.1 & no dock & -65.9 & -72.3 \\
\hline Dracocephalone A & -83.2 & -64.1 & no dock & -76.6 & -82.5 \\
\hline Dracocequinone A & -82.5 & -63.6 & no dock & -77.7 & -74.9 \\
\hline Dracocequinone B & -85.6 & -69.1 & no dock & -76.4 & -75.5 \\
\hline Ferruginol & -78.9 & -71.0 & no dock & -73.2 & -79.0 \\
\hline Hinokiol & -77.7 & -68.5 & no dock & -62.4 & -70.0 \\
\hline Hinokiol-1-one & -84.6 & -69.6 & no dock & -72.9 & -72.4 \\
\hline $7 \beta$-Hydroxyabieta-8,13-diene-11,12-dione & -85.1 & -71.6 & no dock & -73.0 & -85.6 \\
\hline $7 \alpha$-Hydroxyabieta $-8,11,13$-triene & -79.5 & -68.9 & no dock & -67.6 & -77.1 \\
\hline $\begin{array}{l}\text { 14-Hydroxy-7,9(11),13-abietatriene- } 6,12- \\
\text { dione }\end{array}$ & -82.9 & -71.7 & no dock & -71.5 & -84.5 \\
\hline 11-Hydroxy-7,9(11),13-abietatrien-12-one & -81.1 & -68.0 & no dock & -69.6 & -73.3 \\
\hline $\begin{array}{l}\text { 12-Hydroxy-8,12-abietadiene- } 3,11,14- \\
\text { trione }\end{array}$ & -83.4 & -76.4 & no dock & -73.8 & -86.9 \\
\hline $1 \beta$-Hydroxycryptotanshinone & -84.1 & -66.5 & no dock & -75.5 & -88.2 \\
\hline $\begin{array}{l}\text { 7-Hydroxy-12-methoxy-20-nor-abieta- } \\
1,5(10), 7,9,12-\text { pentaen- } 6,14-\text { dione }\end{array}$ & -84.6 & -67.7 & no dock & -73.0 & -78.3 \\
\hline 14-Hydroxy-6-oxoferruginol & -85.2 & -73.0 & no dock & -71.7 & -79.3 \\
\hline 6-Hydroxysalvinolone & -81.2 & -68.6 & no dock & -80.7 & -85.5 \\
\hline Komarovinone A & -86.9 & -66.3 & no dock & -74.9 & -84.7 \\
\hline 1-Oxocryptotanshinone & -83.9 & -66.9 & no dock & -72.6 & -84.1 \\
\hline 1-Oxomiltirone & -85.5 & -64.4 & no dock & -69.7 & -78.6 \\
\hline Royleanone & -82.6 & -72.0 & no dock & -72.8 & -82.1 \\
\hline Sugiol & -81.8 & -70.4 & no dock & -72.6 & -78.7 \\
\hline Taxodione & -81.2 & -68.2 & no dock & -71.1 & -75.8 \\
\hline $\begin{array}{l}6,11,12,16-\text { Tetrahydroxy }-5,8,11,13- \\
\text { abietatetraen-7-one }\end{array}$ & -88.5 & -66.5 & no dock & -85.7 & -93.8 \\
\hline $\begin{array}{l}6,12,14-\text { Trihydroxyabieta }-5,8,11,13- \\
\text { tetraen-7-one }\end{array}$ & -83.5 & -73.4 & no dock & -75.9 & -81.9 \\
\hline 4-epi-Triptobenzene $\mathrm{L}$ & -83.0 & -72.3 & no dock & -65.2 & -76.3 \\
\hline Uncinatone & -93.0 & -73.3 & no dock & -82.5 & -85.5 \\
\hline
\end{tabular}


Table 19. MolDock docking energies (kJ/mol) of clerodane diterpenoids with Leishmania major protein targets.

\begin{tabular}{|c|c|c|c|c|c|c|c|c|c|c|c|c|}
\hline Clerodane diterpenoids & LmajCatB & LmajDHODH & LmajdUTPase & LmajNDKb & LmajNH & LdonNMT & LmajOPB & LmajPDE1 & LmajPTR1 & LmajMetRS & LmajTyrRS & LmajUGPase \\
\hline 15-Acetoxy-cis-cleroden-3-en-18-al & -68.6 & -94.1 & -85.0 & -97.9 & -96.0 & -87.3 & -90.4 & -94.9 & -94.7 & -107.1 & -92.6 & -106.9 \\
\hline 15-Acetoxy-cis-cleroden-3-en-18-oic acid & -75.0 & -103.1 & -88.3 & -97.2 & -92.3 & -87.0 & -91.6 & -95.3 & -93.2 & -97.7 & -94.4 & -97.3 \\
\hline 18-Acetoxy-cis-cleroden-3-en-15-oic acid & -76.9 & -103.7 & -82.7 & -96.7 & -88.8 & -80.7 & -97.3 & -96.8 & -89.3 & -104.2 & -97.5 & -98.2 \\
\hline 15-O-Acetylcistadiol & -68.0 & -94.7 & -87.4 & -97.3 & -94.7 & -80.4 & -96.8 & -89.2 & -92.9 & -106.7 & -95.0 & -95.3 \\
\hline 18-O-Acetylcistadiol & -70.5 & -103.4 & -88.1 & -91.7 & -93.2 & -85.0 & -94.0 & -93.8 & -82.3 & -101.2 & -95.9 & -100.3 \\
\hline Cistadiol & -64.1 & -93.4 & -79.7 & -81.6 & -81.9 & -69.0 & -77.1 & -85.2 & -79.2 & -78.9 & -80.7 & -92.5 \\
\hline trans-Dehydrocrotonin & -78.6 & -114.9 & -90.2 & -91.8 & -84.5 & -75.9 & -89.0 & -89.1 & -99.2 & -115.1 & -97.7 & -92.1 \\
\hline $15,18-\mathrm{Di}-O$-acetylcistadiol & -77.8 & -100.5 & -92.8 & -109.4 & -94.8 & -84.2 & -90.5 & -107.0 & -91.6 & -117.5 & -100.6 & -110.4 \\
\hline 8-epi-Kolavenol & -71.5 & -94.5 & -79.2 & -80.4 & -85.0 & -72.3 & -78.7 & -80.6 & -82.8 & -92.9 & -85.3 & -95.3 \\
\hline epi-Populifolic acid & -69.9 & -85.5 & -72.7 & -82.3 & -83.4 & -71.7 & -76.3 & -82.2 & -78.3 & -85.0 & -78.2 & -89.9 \\
\hline ent-16R-Hydroxy-3,13-clerodadien-15,16-olide & -77.7 & -108.7 & -86.4 & -88.2 & -87.7 & -88.2 & -90.8 & -87.5 & -101.9 & -105.9 & -104.9 & -97.4 \\
\hline ent-16S-Hydroxy-3,13-clerodadien-15,16-olide & -81.9 & -101.9 & -84.6 & -91.0 & -83.9 & -78.4 & -93.4 & -82.2 & -100.3 & -110.7 & -99.4 & -105.1 \\
\hline 15-Hydroxy-cis-cleroden-3-en-18-al & -66.5 & -91.8 & -77.4 & -81.8 & -86.8 & -76.7 & -76.8 & -83.7 & -77.5 & -92.8 & -80.1 & -91.4 \\
\hline 15-Hydroxy-cis-cleroden-3-en-18-oic acid & -69.0 & -95.8 & -90.1 & -83.5 & -86.9 & -80.5 & -82.1 & -86.0 & -80.4 & -95.9 & -84.3 & -95.2 \\
\hline 18-Hydroxy-cis-cleroden-3-en-15-oic acid & -70.0 & -93.4 & -76.1 & -86.3 & -86.8 & -76.2 & -79.7 & -87.2 & -81.1 & -96.1 & -82.9 & -97.4 \\
\hline ent-12-Oxo-3,13(16)-clerodien-15-oic acid & -75.0 & -112.7 & -86.3 & -90.0 & -93.2 & -88.2 & -87.7 & -83.0 & -96.5 & -107.2 & -87.6 & -110.5 \\
\hline
\end{tabular}


Table 20. MolDock docking energies (kJ/mol) of clerodane diterpenoids with Leishmania donovani and L. mexicana protein targets.

\begin{tabular}{|c|c|c|c|c|c|c|c|c|c|c|c|c|}
\hline \multirow{2}{*}{ Clerodane diterpenoids } & LdonCatB & LdonCyp & LdonDHODH & LdonNMT & LmexGAPDH & LmexGPDH & LmexPGI & LmexPMM & \multirow{2}{*}{$\begin{array}{r}\text { LmexPYK } \\
\text { Site } 1 \\
\end{array}$} & \multirow{2}{*}{$\begin{array}{r}\text { LmexPYK } \\
\text { Site } 2 \\
\end{array}$} & \multirow{2}{*}{$\begin{array}{r}\text { LmexPYK } \\
\text { Site } 3 \\
\end{array}$} & \multirow[t]{2}{*}{ LmexTIM } \\
\hline & & & & & & & & & & & & \\
\hline 15-Acetoxy-cis-cleroden-3-en-18-al & -80.4 & -84.1 & -75.1 & -87.3 & -76.7 & -107.2 & -75.4 & -101.2 & -96.3 & -96.5 & -89.3 & -84.6 \\
\hline 15-Acetoxy-cis-cleroden-3-en-18-oic acid & -83.5 & -85.0 & -75.2 & -87.0 & -86.3 & -93.6 & -84.4 & -104.8 & -100.2 & -92.9 & -90.4 & -80.9 \\
\hline 18-Acetoxy-cis-cleroden-3-en-15-oic acid & -82.9 & -90.6 & -85.1 & -80.7 & -77.3 & -109.2 & -77.6 & -97.4 & -95.9 & -90.8 & -89.5 & -95.5 \\
\hline 15-O-Acetylcistadiol & -82.8 & -79.6 & -72.7 & -80.4 & -77.4 & -102.7 & -77.8 & -98.9 & -96.3 & -96.8 & -87.7 & -89.7 \\
\hline 18-O-Acetylcistadiol & -79.8 & -92.7 & -66.9 & -85.0 & -73.2 & -105.6 & -78.9 & -97.1 & -92.5 & -96.8 & -92.6 & -98.6 \\
\hline trans-Dehydrocrotonin & -92.8 & -98.7 & -51.3 & -75.9 & -73.0 & -100.5 & -77.1 & -94.2 & -107.8 & -87.6 & -92.4 & -95.2 \\
\hline $15,18-\mathrm{Di}-O-$ acetylcistadiol & -82.2 & -82.4 & -94.8 & -84.2 & -85.2 & -122.2 & -86.7 & -104.1 & -102.9 & -101.7 & -97.4 & -99.1 \\
\hline 8-epi-Kolavenol & -75.5 & -83.0 & -77.8 & -72.3 & -71.7 & -100.8 & -70.6 & -85.6 & -92.1 & -84.2 & -94.6 & -78.9 \\
\hline epi-Populifolic acid & -68.3 & -75.8 & -63.4 & -71.7 & -73.8 & -89.4 & -69.7 & -88.7 & -91.1 & -79.3 & -85.0 & -82.4 \\
\hline ent-16R-Hydroxy-3,13-clerodadien-15,16-olide & -74.7 & -89.1 & -82.9 & -88.2 & -85.4 & -105.8 & -79.8 & -96.5 & -103.1 & -89.0 & -95.5 & -83.1 \\
\hline ent-16S-Hydroxy-3,13-clerodadien-15,16-olide & -88.6 & -91.8 & -82.7 & -78.4 & -83.9 & -100.1 & -81.3 & -97.2 & -102.0 & -92.3 & -90.3 & -85.8 \\
\hline 15-Hydroxy-cis-cleroden-3-en-18-oic acid & -71.4 & -86.4 & -66.4 & -80.5 & -74.9 & -99.5 & -73.3 & -85.9 & -99.3 & -88.1 & -88.5 & -80.5 \\
\hline 18-Hydroxy-cis-cleroden-3-en-15-oic acid & -72.5 & -84.3 & -59.0 & -76.2 & -78.2 & -95.4 & -73.2 & -90.2 & -89.2 & -83.9 & -90.5 & -88.0 \\
\hline ent $-12-$ - xo-3,13(16)-clerodien-15-oic acid & -84.3 & -95.2 & -72.0 & -88.2 & -85.3 & -88.0 & -76.6 & -95.9 & -108.8 & -88.3 & -91.6 & -86.9 \\
\hline
\end{tabular}


Table 21. MolDock docking energies $(\mathrm{kJ} / \mathrm{mol})$ of clerodane and labdane diterpenoids with Leishmania infantum protein targets.

\begin{tabular}{|c|c|c|c|c|c|}
\hline Clerodane diterpenoids & LinfCYP51 & LinfGLO2 & LinfPnC1 & LinfTDR1 & LinfTR \\
\hline 15-Acetoxy-cis-cleroden-3-en-18-al & -96.8 & -78.2 & no dock & -90.4 & -91.8 \\
\hline 15-Acetoxy-cis-cleroden-3-en-18-oic acid & -93.0 & -82.7 & no dock & -87.6 & -91.7 \\
\hline 18-Acetoxy-cis-cleroden-3-en-15-oic acid & -98.2 & -78.1 & no dock & -87.6 & -97.6 \\
\hline 15-O-Acetylcistadiol & -101.7 & -89.8 & no dock & -88.4 & -94.3 \\
\hline 18-O-Acetylcistadiol & -95.8 & -78.3 & no dock & -88.4 & -93.8 \\
\hline Cistadiol & -86.8 & -68.7 & no dock & -78.3 & -82.2 \\
\hline trans-Dehydrocrotonin & -89.6 & -74.5 & no dock & -77.6 & -88.9 \\
\hline $15,18-\mathrm{Di}-O-$ acetylcistadiol & -95.6 & -85.8 & no dock & -89.2 & -103.5 \\
\hline 8-epi-Kolavenol & -83.6 & -72.3 & -18.2 & -80.5 & -78.8 \\
\hline epi-Populifolic acid & -83.8 & -71.4 & no dock & -75.9 & -77.0 \\
\hline ent-16R-Hydroxy-3,13-clerodadien-15,16-olide & -87.9 & -75.1 & -22.6 & -83.9 & -87.5 \\
\hline ent-16S-Hydroxy-3,13-clerodadien-15,16-olide & -88.4 & -67.5 & no dock & -79.6 & -78.8 \\
\hline 15-Hydroxy-cis-cleroden-3-en-18-al & -83.0 & -68.9 & no dock & -81.0 & -81.2 \\
\hline 15-Hydroxy-cis-cleroden-3-en-18-oic acid & -89.4 & -66.8 & no dock & -78.4 & -78.1 \\
\hline 18-Hydroxy-cis-cleroden-3-en-15-oic acid & -87.5 & -69.6 & -31.7 & -82.8 & -81.3 \\
\hline \multicolumn{6}{|l|}{ Labdane diterpenoids } \\
\hline ent $-3 \beta$-Acetoxy-13-epi-manoyl-oxide & -94.3 & -72.4 & no dock & -67.4 & -88.0 \\
\hline Andrographolide & -97.9 & -80.4 & no dock & -81.1 & -91.9 \\
\hline \multicolumn{6}{|l|}{ 14(R)-Aulacocarpin C } \\
\hline 14(S)-Aulacocarpin C & -93.1 & -81.7 & no dock & -81.7 & -92.8 \\
\hline Aulacocarpin D & -92.7 & -74.5 & no dock & -85.8 & -93.5 \\
\hline trans-Communic acid & -90.8 & -75.0 & no dock & -82.6 & -91.6 \\
\hline trans-Communic acid methyl ester & -84.3 & -77.2 & no dock & -76.0 & -82.7 \\
\hline Copalic acid & -89.8 & -74.8 & no dock & -72.8 & -86.8 \\
\hline Dehydropinifolic acid 15-methyl ester & -93.9 & -84.1 & no dock & -78.1 & -83.6 \\
\hline $\begin{array}{l}\text { 12(S)-Hydroxy-15(R)-methoxy-labdan- } \\
8(17), 13(14)-\text { dien }-15,16 \text {-olide }\end{array}$ & -93.3 & -84.8 & no dock & -89.8 & -93.5 \\
\hline $\begin{array}{l}\text { 12(S)-Hydroxy-15(S)-methoxy-labdan- } \\
8(17,) 13(14)-\text { dien}-15,16 \text {-olide }\end{array}$ & -99.3 & -88.5 & no dock & -88.8 & -95.1 \\
\hline Labda-8(17),12-diene-15,16-dial & -94.2 & -84.8 & no dock & -89.2 & -100.2 \\
\hline 13(E)-Labda-7,13-dien-8a,15-diol & -87.7 & -76.3 & no dock & -81.7 & -86.6 \\
\hline Labda-12,14-dien-7 $-8,8 \alpha-$ diol & -89.1 & -77.8 & no dock & -79.8 & -88.4 \\
\hline Labdan-8a,15-diol & -85.9 & -77.1 & no dock & -76.1 & -87.2 \\
\hline 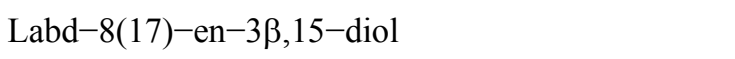 & -85.8 & -77.4 & no dock & -82.0 & -90.2 \\
\hline 13(E)-Labd-13-en-8 $\alpha, 15$-diol & -84.2 & -76.9 & no dock & -79.7 & -87.2 \\
\hline Lambertianic acid & -87.0 & -75.4 & no dock & -78.4 & -83.4 \\
\hline $\begin{array}{l}15(R)-\text { Methoxy-labdan-8(17),11(E),13(14)- } \\
\text { trien-15,16-olide }\end{array}$ & -90.0 & -73.6 & no dock & -72.5 & -89.3 \\
\hline $\begin{array}{l}\text { 15(S)-Methoxy-labdan-8(17),11(E),13(14)- } \\
\text { trien-15,16-olide }\end{array}$ & -94.2 & -87.8 & no dock & -88.3 & -92.3 \\
\hline ent-12-Oxo-8,13(16)-labdadien-15-oic acid & -96.0 & -86.3 & no dock & -85.3 & -96.8 \\
\hline ent $-3 \beta-$ Acetoxy-13-epi-manoyl-oxide & -94.1 & -77.8 & no dock & -78.5 & -88.9 \\
\hline
\end{tabular}


Table 22. MolDock docking energies $(\mathrm{kJ} / \mathrm{mol})$ of labdane diterpenoids with Leishmania major protein targets.

\begin{tabular}{|c|c|c|c|c|c|c|c|c|c|c|c|c|}
\hline Labdane diterpenoids & LmajCatB & LmajDHODH & LmajdUTPase & LmajNDKb & LmajNH & LdonNMT & LmajOPB & LmajPDE1 & LmajPTR1 & LmajMetRS & LmajTyrRS & LmajUGPase \\
\hline ent $-3 \beta-$ Acetoxy-13-epi-manoyl-oxide & -74.9 & -96.4 & -76.3 & -82.2 & -82.7 & -78.7 & -90.7 & -82.0 & -89.0 & -85.9 & -80.1 & -91.2 \\
\hline Andrographolide & -86.7 & -114.6 & -90.5 & -92.1 & -96.5 & -101.8 & -98.5 & -94.4 & -101.6 & -114.1 & -97.7 & -95.9 \\
\hline 14(R)-Aulacocarpin C & -74.4 & -116.1 & -85.2 & -91.7 & -97.7 & -99.7 & -95.7 & -85.2 & -98.1 & -107.0 & -91.8 & -103.3 \\
\hline $14(S)$-Aulacocarpin C & -83.6 & -114.7 & -88.3 & -90.3 & -91.7 & -97.4 & -90.6 & -83.1 & -99.9 & -107.9 & -94.0 & -100.3 \\
\hline Aulacocarpin D & -80.8 & -108.3 & -89.0 & -87.3 & -87.3 & -86.0 & -91.8 & -98.6 & -92.9 & -108.3 & -93.8 & -98.9 \\
\hline trans-Communic acid & -69.5 & -109.4 & -86.3 & -86.7 & -87.5 & -89.7 & -83.7 & -84.0 & -88.4 & -97.1 & -88.2 & -88.3 \\
\hline trans - Communic acid methyl ester & -81.3 & -103.5 & -84.7 & -86.3 & -85.6 & -84.1 & -85.7 & -83.0 & -92.3 & -99.6 & -89.8 & -88.7 \\
\hline Copalic acid & -69.5 & -102.7 & -88.4 & -89.5 & -83.2 & -83.9 & -86.0 & -96.2 & -90.1 & -105.1 & -93.9 & -104.6 \\
\hline Dehydropinifolic acid 15-methyl ester & -87.3 & -116.3 & -102.2 & -90.1 & -87.9 & -91.3 & -101.0 & -89.8 & -89.7 & -104.1 & -99.8 & -103.3 \\
\hline $\begin{array}{l}\text { 12(S)-Hydroxy-15(R)-methoxy-labdan- } \\
8(17), 13(14) \text {-dien-15,16-olide }\end{array}$ & -85.8 & -114.5 & -87.2 & -97.5 & -88.7 & -93.8 & -96.4 & -88.0 & -96.1 & -108.9 & -103.0 & -110.1 \\
\hline $\begin{array}{l}\text { 12(S)-Hydroxy-15(S)-methoxy-labdan- } \\
8(17,) 13(14) \text {-dien-15,16-olide }\end{array}$ & -90.2 & -112.6 & -89.4 & -98.7 & -98.8 & -84.0 & -107.3 & -89.1 & -91.2 & -110.4 & -103.6 & -111.4 \\
\hline Labda-8(17),12-diene-15,16-dial & -77.5 & -107.2 & -87.2 & -82.9 & -93.0 & -95.8 & -82.8 & -89.0 & -97.5 & -103.2 & -95.5 & -102.8 \\
\hline 13(E)-Labda-7,13-dien-8a,15-diol & -76.9 & -100.6 & -85.8 & -87.0 & -86.3 & -89.7 & -100.7 & -85.8 & -87.9 & -107.5 & -91.0 & -90.8 \\
\hline 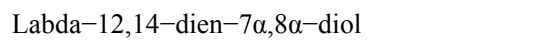 & -71.9 & -99.4 & -76.2 & -75.8 & -89.8 & -89.8 & -90.5 & -85.1 & -81.5 & -101.3 & -87.8 & -94.7 \\
\hline Labdan-8 $\alpha, 15$-diol & -78.9 & -101.4 & -84.6 & -81.5 & -80.3 & -92.1 & -93.7 & -82.7 & -86.0 & -108.6 & -83.9 & -92.3 \\
\hline Labd-8(17)-en-3 $\beta, 15$-diol & -75.5 & -106.7 & -82.2 & -82.9 & -80.6 & -71.0 & -88.0 & -81.0 & -93.9 & -104.9 & -85.9 & -90.8 \\
\hline 13(E)-Labd-13-en-8a,15-diol & -69.4 & -105.0 & -82.1 & -83.3 & -85.1 & -89.1 & -88.5 & -85.8 & -88.9 & -107.8 & -89.8 & -92.2 \\
\hline Lambertianic acid & -69.8 & -110.5 & -86.3 & -82.0 & -84.0 & -79.8 & -81.8 & -83.9 & -93.5 & -100.7 & -87.7 & -88.9 \\
\hline $\begin{array}{l}\text { 15(R)-Methoxy-labdan-8(17),11(E),13(14)- } \\
\text { trien-15,16-olide }\end{array}$ & -88.3 & -111.4 & -84.5 & -97.3 & -91.3 & -91.7 & -104.9 & -94.4 & -91.8 & -101.5 & -95.0 & -98.9 \\
\hline $\begin{array}{l}\text { 15(S)-Methoxy-labdan-8(17),11(E),13(14)- } \\
\text { trien-15,16-olide }\end{array}$ & -83.1 & -104.7 & -84.8 & -89.0 & -95.4 & -88.5 & -109.2 & -98.3 & -99.0 & -106.2 & -98.3 & -97.3 \\
\hline $\begin{array}{l}\text { ent-12-Oxo-8,13(16)-labdadien-15-oic } \\
\text { acid }\end{array}$ & -78.0 & -107.7 & -93.4 & -92.3 & -88.2 & -92.1 & -90.6 & -90.0 & -94.1 & -100.0 & -97.0 & -100.4 \\
\hline
\end{tabular}


Table 23. MolDock docking energies (kJ/mol) of labdane diterpenoids with Leishmania donovani and L. mexicana protein targets.

\begin{tabular}{|c|c|c|c|c|c|c|c|c|c|c|c|c|}
\hline Labdane diterpenoids & LdonCatB & LdonCyp & LdonDHODH & LdonNMT & LmexGAPDH & LmexGPDH & LmexPGI & LmexPMM & $\begin{array}{c}\text { LmexPYK } \\
\text { Site } 1 \\
\end{array}$ & $\begin{array}{l}\text { LmexPYK } \\
\text { Site } 2 \\
\end{array}$ & $\begin{array}{c}\text { LmexPYK } \\
\text { Site } 3 \\
\end{array}$ & LmexTIM \\
\hline ent $-3 \beta-$ Acetoxy-13-epi-manoyl-oxide & -83.3 & -77.6 & -60.4 & -78.7 & -67.9 & -94.4 & -69.1 & -86.9 & -90.2 & -84.0 & -77.7 & -78.3 \\
\hline Andrographolide & -88.9 & -93.2 & -94.9 & -101.8 & -86.1 & -114.0 & -79.6 & -100.7 & -110.3 & -100.3 & -107.0 & -95.5 \\
\hline 14(R)-Aulacocarpin C & -87.0 & -98.3 & -92.5 & -99.7 & -78.4 & -103.3 & -76.8 & -95.7 & -99.6 & -91.7 & -114.0 & -84.2 \\
\hline 14(S)-Aulacocarpin C & -85.6 & -95.6 & -90.3 & -97.4 & -75.0 & -100.7 & -77.8 & -90.6 & -97.1 & -95.8 & -109.0 & -86.7 \\
\hline Aulacocarpin D & -96.6 & -88.1 & -75.2 & -86.0 & -87.6 & -99.9 & -73.6 & -87.5 & -95.9 & -89.8 & -88.3 & -87.8 \\
\hline trans-Communic acid & -77.9 & -87.9 & -79.6 & -89.7 & -70.6 & -100.8 & -75.8 & -88.3 & -92.3 & -82.9 & -98.3 & -83.1 \\
\hline trans-Communic acid methyl ester & -85.8 & -90.8 & -81.9 & -84.1 & -73.5 & -104.8 & -72.2 & -88.1 & -95.4 & -82.4 & -90.0 & -85.2 \\
\hline Copalic acid & -89.0 & -92.6 & -85.1 & -83.9 & -73.8 & -90.3 & -76.9 & -92.0 & -94.8 & -88.0 & -92.4 & -97.5 \\
\hline Dehydropinifolic acid 15-methyl ester & -85.8 & -95.2 & -88.4 & -91.3 & -84.5 & -123.2 & -85.7 & -100.6 & -111.6 & -93.2 & -104.2 & -99.0 \\
\hline $\begin{array}{l}\text { 12(S)-Hydroxy-15(R)-methoxy-labdan- } \\
\text { 8(17),13(14)-dien-15,16-olide }\end{array}$ & -92.1 & -93.8 & -90.8 & -93.8 & -75.2 & -117.2 & -79.4 & -101.2 & -117.2 & -96.5 & -104.7 & -93.8 \\
\hline $\begin{array}{l}\text { 12(S)-Hydroxy-15(S)-methoxy-labdan- } \\
8(17,) 13(14)-\text { dien-15,16-olide }\end{array}$ & -95.4 & -92.4 & -82.7 & -84.0 & -88.1 & -116.2 & -91.2 & -103.1 & -117.1 & -92.4 & -109.7 & -93.1 \\
\hline Labda-8(17),12-diene-15,16-dial & -90.6 & -93.4 & -85.7 & -95.8 & -75.4 & -97.3 & -72.3 & -93.0 & -97.0 & -87.4 & -99.5 & -85.4 \\
\hline 13(E)-Labda-7,13-dien-8a,15-diol & -88.4 & -82.3 & -80.9 & -89.7 & -76.3 & -109.7 & -73.5 & -87.1 & -100.2 & -82.1 & -93.7 & -88.8 \\
\hline 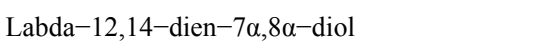 & -86.9 & -83.8 & -80.5 & -89.8 & -77.9 & -86.3 & -78.7 & -89.2 & -100.4 & -85.5 & -91.2 & -91.6 \\
\hline Labdan-8 $\alpha, 15-$ diol & -86.8 & -82.0 & -82.5 & -92.1 & -73.2 & -94.9 & -76.4 & -95.9 & -93.7 & -86.0 & -90.8 & -88.8 \\
\hline Labd-8(17)-en-3ß,15-diol & -78.5 & -85.4 & -86.9 & -71.0 & -71.0 & -87.8 & -72.0 & -93.5 & -97.3 & -81.6 & -93.8 & -86.2 \\
\hline 13(E)-Labd-13-en-8 $\alpha, 15$-diol & -81.4 & -82.1 & -77.1 & -89.1 & -71.9 & -104.1 & -83.4 & -88.7 & -99.2 & -84.5 & -92.7 & -92.0 \\
\hline Lambertianic acid & -82.2 & -82.9 & -81.6 & -79.8 & -72.9 & -109.5 & -75.1 & -95.1 & -96.2 & -85.6 & -98.4 & -80.3 \\
\hline $\begin{array}{l}\text { 15(R)-Methoxy-labdan-8(17),11(E),13(14)- } \\
\text { trien-15,16-olide }\end{array}$ & -106.6 & -91.2 & -92.5 & -91.7 & -79.3 & -121.2 & -76.7 & -97.1 & -105.9 & -94.3 & -95.6 & -91.2 \\
\hline $\begin{array}{l}\text { 15(S)-Methoxy-labdan-8(17),11(E),13(14)- } \\
\text { trien-15,16-olide }\end{array}$ & -92.3 & -92.2 & -93.3 & -88.5 & -87.3 & -115.8 & -78.5 & -94.5 & -101.8 & -97.0 & -89.2 & -91.5 \\
\hline $\begin{array}{l}\text { ent-12-Oxo-8,13(16)-labdadien-15-oic } \\
\text { acid }\end{array}$ & -76.2 & -84.3 & -84.0 & -92.1 & -89.2 & -99.0 & -72.6 & -102.2 & -105.2 & -91.1 & -103.5 & -91.8 \\
\hline
\end{tabular}


Table 24. MolDock docking energies ( $\mathrm{kJ} / \mathrm{mol})$ of kaurane and pimarane diterpenoids with Leishmania major protein targets.

\begin{tabular}{|c|c|c|c|c|c|c|c|c|c|c|c|c|}
\hline Kaurane diterpenoids & LmajCatB & LmajDHODH & LmajdUTPase & LmajNDKb & LmajNH & LdonNMT & LmajOPB & LmajPDE1 & LmajPTR1 & LmajMetRS & LmajTyrRS & LmajUGPase \\
\hline 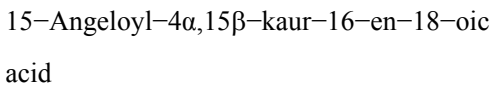 & -88.6 & -116.3 & -75.8 & -97.5 & -97.3 & -78.2 & -85.5 & -98.6 & -97.4 & -116.0 & -91.4 & -106.3 \\
\hline ent $-11 \alpha-$ Hydroxy-16-kauren-15-one & -64.3 & -98.8 & -69.7 & -76.7 & -83.3 & -64.7 & -87.8 & -83.0 & -74.0 & -85.7 & -75.1 & -81.0 \\
\hline Kaurenoic acid & -70.4 & -95.2 & -77.8 & -76.3 & -81.4 & -58.4 & -86.5 & -80.6 & -89.7 & -97.5 & -75.5 & -83.2 \\
\hline Perymenic acid & -97.6 & -115.4 & -75.2 & -95.4 & -103.0 & -81.2 & -88.9 & -99.6 & -98.5 & -118.4 & -91.2 & -103.7 \\
\hline $\begin{array}{l}\text { ent }-15 \beta-\text { Senecioyloxy-16,17-epoxy- } \\
\text { kauran-18-oic acid }\end{array}$ & -97.3 & -120.5 & -76.6 & -93.6 & -102.4 & -87.8 & -100.7 & -100.8 & -86.6 & -111.7 & -93.3 & -104.6 \\
\hline \multicolumn{13}{|l|}{ Pimarane diterpenoids } \\
\hline Acanthoic acid & -66.0 & -97.0 & -73.1 & -84.0 & -86.6 & -72.3 & -87.9 & -81.1 & -78.7 & -87.9 & -72.7 & -82.5 \\
\hline $\begin{array}{l}\text { 7ß-Hydroxy-ent-pimara-8(14),15- } \\
\text { dien-19-oic acid }\end{array}$ & -74.0 & -93.7 & -79.3 & -84.3 & -87.3 & -71.7 & -92.6 & -87.7 & -76.7 & -87.6 & -78.9 & -81.2 \\
\hline ent-15-Pimarene-8 $\beta, 19$-diol & -72.1 & -97.1 & -69.0 & -78.0 & -86.3 & -66.1 & -87.4 & -79.1 & -77.5 & -84.4 & -74.3 & -85.5 \\
\hline ent-8(14),15-Pimaradien-3 $\beta$-acetoxy & -72.5 & -85.3 & -83.9 & -90.3 & -89.4 & -71.7 & -88.6 & -82.1 & -83.7 & -99.7 & -88.9 & -92.1 \\
\hline ent-8(14),15-Pimaradien-3 $\beta, 19$-diol & -66.9 & -90.8 & -66.2 & -78.3 & -91.3 & -71.5 & -83.3 & -80.0 & -75.0 & -86.6 & -80.9 & -81.6 \\
\hline ent-Pimara-8(14),15-dien-19-oic acid & -65.8 & -91.7 & -69.9 & -81.3 & -87.1 & -70.6 & -89.3 & -80.2 & -81.5 & -85.7 & -75.7 & -77.4 \\
\hline ent-8(14),15-Pimaradien-3 $3-\mathrm{ol}$ & -64.7 & -89.0 & -66.5 & -77.7 & -86.2 & -66.6 & -82.3 & -76.4 & -73.8 & -79.4 & -81.8 & -76.4 \\
\hline
\end{tabular}


Table 25. MolDock docking energies $(\mathrm{kJ} / \mathrm{mol})$ of kaurane and pimarane diterpenoids with Leishmania donovani and L. mexicana protein targets.

\begin{tabular}{|c|c|c|c|c|c|c|c|c|c|c|c|c|}
\hline Kaurane diterpenoids & LdonCatB & LdonCyp & LdonDHODH & LdonNMT & LmexGAPDH & LmexGPDH & LmexPGI & LmexPMM & $\begin{array}{c}\text { LmexPYK } \\
\text { Site } 1 \\
\end{array}$ & $\begin{array}{c}\text { LmexPYK } \\
\text { Site } 2 \\
\end{array}$ & $\begin{array}{c}\text { LmexPYK } \\
\text { Site } 3 \\
\end{array}$ & LmexTIM \\
\hline 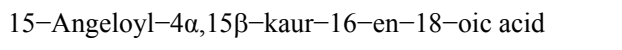 & -97.4 & -78.7 & -27.8 & -78.2 & -91.8 & -121.4 & -75.9 & -96.9 & -104.0 & -89.2 & -92.8 & -77.3 \\
\hline ent-11 $\alpha$-Hydroxy-16-kauren-15-one & -66.2 & -82.3 & no dock & -64.7 & -61.9 & -84.4 & -63.0 & -81.1 & -79.6 & -73.8 & -70.1 & -80.5 \\
\hline Kaurenoic acid & -69.6 & -73.9 & -21.6 & -58.4 & -64.6 & -88.8 & -64.5 & -80.3 & -93.9 & -77.4 & -81.3 & -78.1 \\
\hline Perymenic acid & -100.6 & -92.3 & -45.0 & -81.2 & -85.7 & -128.3 & -76.8 & -100.4 & -99.7 & -95.7 & -91.9 & -80.3 \\
\hline $\begin{array}{l}\text { ent-15ß-Senecioyloxy-16,17-epoxy-kauran-18-oic } \\
\text { acid }\end{array}$ & -99.1 & -88.1 & -38.0 & -87.8 & -90.1 & -128.9 & -77.2 & -96.5 & -99.3 & -92.9 & -98.0 & -81.1 \\
\hline \multicolumn{13}{|l|}{ Pimarane diterpenoids } \\
\hline Acanthoic_acid & -69.3 & -79.7 & -62.5 & -72.3 & -59.8 & -90.8 & -69.0 & -81.5 & -92.4 & -79.2 & -78.3 & -79.4 \\
\hline $\begin{array}{l}\text { 7ß-Hydroxy-ent-pimara-8(14),15-dien-19-oic } \\
\text { acid }\end{array}$ & -71.2 & -78.8 & -13.1 & -71.7 & -71.7 & -101.0 & -68.7 & -88.0 & -96.6 & -83.5 & -76.9 & -71.3 \\
\hline ent-15-Pimarene-8 8,19 -diol & -75.9 & -77.9 & -63.4 & -66.1 & -72.6 & -85.2 & -68.6 & -79.0 & -79.7 & -80.1 & -71.6 & -79.0 \\
\hline ent-8(14),15-Pimaradien-3 3 -acetoxy & -79.6 & -80.5 & -36.8 & -71.7 & -71.4 & -90.2 & -63.2 & -83.7 & -88.7 & -84.4 & -73.5 & -76.4 \\
\hline ent-8(14),15-Pimaradien-3 $\beta, 19$-diol & -67.6 & -73.6 & -31.7 & -71.5 & -65.3 & -90.2 & -69.2 & -83.6 & -95.0 & -79.6 & -73.6 & -82.7 \\
\hline ent-Pimara-8(14),15-dien-19-oic acid & -66.6 & -77.7 & -53.0 & -70.6 & -64.0 & -94.0 & -66.0 & -85.4 & -88.0 & -79.7 & -70.8 & -77.1 \\
\hline ent-8(14),15-Pimaradien-3b-ol & -69.3 & -71.6 & -26.5 & -66.6 & -63.1 & -88.7 & -64.5 & -80.7 & -94.3 & -77.1 & -72.6 & -79.9 \\
\hline
\end{tabular}


Table 26. MolDock docking energies (kJ/mol) of miscellaneous diterpenoids with Leishmania major protein targets.

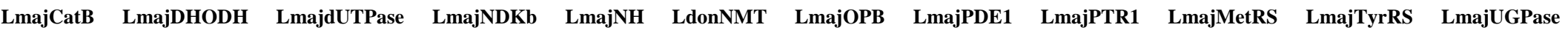

Cassane diterpenoids

\begin{abstract}
$6 \beta$-O-Cinnamoyl-12-hydroxy-
\end{abstract}

$-78.8$

$-110.4$

$-111$.

$-119.5$

$-108.1$

$-107.4$

$-108.1$

$-120.0$

$-134.4$

$-115.8$

$-116.4$

$6 \alpha, 7 \beta$-Diacetoxyvouacapane

$\begin{array}{ll}-77.8 & -118.7\end{array}$

$-80.7$

$-91.0$

$-92.6-74.7$

$-85.6$

$-99.8$

$-86.5$

$-90.4$

$-88.3$

$-88.0$

hydroxy-(13)15-en-16,12-olide-18

$-107.0 \quad-125.0$

$-97.1$

$-107$.

$-121.6-101$

$-109.0 \quad-109$.

$-117.3$

$-135.2$

$-115.1$

$-114.3$

cassaneoic acid
Icetaxane diterpenoids

\begin{tabular}{|c|c|c|c|c|c|c|c|c|c|c|c|c|}
\hline Cyclocoulterone & -70.3 & -92.1 & -83.7 & -88.6 & -78.7 & -81.3 & -86.7 & -96.1 & -91.6 & -100.2 & -91.8 & -92.0 \\
\hline 5-epi-Icetexone & -80.9 & -91.0 & -74.2 & -81.6 & -88.3 & -27.2 & -95.7 & -88.5 & -88.5 & -96.9 & -98.8 & -85.9 \\
\hline Komaroviquinone & -75.7 & -83.0 & -87.6 & -84.9 & -75.6 & -76.5 & -90.9 & -98.1 & -97.8 & -105.2 & -87.8 & -87.0 \\
\hline \multicolumn{13}{|l|}{ Mulinane diterpenoids } \\
\hline Azorellanol & -64.7 & -107.8 & -74.6 & -79.1 & -98.7 & -80.0 & -94.5 & -99.7 & -85.3 & -98.6 & -88.5 & -91.9 \\
\hline 7-Deacetylazorellanol & -72.5 & -98.4 & -72.3 & -80.2 & -79.0 & -72.4 & -88.9 & -91.8 & -76.2 & -94.0 & -90.1 & -78.7 \\
\hline $\begin{array}{l}\text { Mulin-11,13-dien-20-oic } \\
\text { acid }\end{array}$ & -83.1 & -103.0 & -78.0 & -84.3 & -80.7 & -71.5 & -87.5 & -83.5 & -87.0 & -100.5 & -88.1 & -88.3 \\
\hline Mulinic acid & -69.4 & -98.9 & -71.9 & -82.4 & -80.7 & -82.3 & -83.6 & -85.7 & -74.6 & -93.7 & -78.3 & -97.0 \\
\hline Mulinolic acid & -75.6 & -94.5 & -87.3 & -82.5 & -84.5 & -85.1 & -84.9 & -90.2 & -81.1 & -102.3 & -92.2 & -88.7 \\
\hline \multicolumn{13}{|l|}{ Miscellaneous diterpenoids } \\
\hline Hypoestoxide & -74.1 & -117.7 & -88.3 & -80.1 & -87.8 & -85.2 & -94.0 & -97.0 & -78.1 & -81.1 & -99.5 & -99.2 \\
\hline Komarovispirone & -74.1 & -92.9 & -75.8 & -88.9 & -90.5 & -75.5 & -85.4 & -86.4 & -75.3 & -79.9 & -94.0 & -91.4 \\
\hline Sacculatal & -69.5 & -112.7 & -85.2 & -87.1 & -86.1 & -82.4 & -88.0 & -85.9 & -104.4 & -103.5 & -95.2 & -100.4 \\
\hline Serratol & -69.2 & -90.5 & -75.7 & -77.2 & -78.8 & -77.4 & -82.1 & -89.7 & -79.1 & -93.2 & -90.3 & -88.6 \\
\hline Totarol & -62.7 & -96.6 & -64.9 & -85.1 & -76.4 & -69.8 & -89.2 & -80.7 & -74.6 & -97.7 & -77.8 & -93.1 \\
\hline
\end{tabular}


Table 27. MolDock docking energies $(\mathrm{kJ} / \mathrm{mol})$ of miscellaneous diterpenoids with Leishmania donovani and L. mexicana protein targets.

\begin{tabular}{|c|c|c|c|c|c|c|c|c|c|c|c|c|}
\hline Cassane diterpenoids & LdonCatB & LdonCyp & LdonDHODH & LdonNMT & LmexGAPDH & LmexGPDH & LmexPGI & LmexPMM & $\begin{array}{c}\text { LmexPYK } \\
\text { Site } 1 \\
\end{array}$ & $\begin{array}{c}\text { LmexPYK } \\
\text { Site } 2 \\
\end{array}$ & $\begin{array}{c}\text { LmexPYK } \\
\text { Site } 3 \\
\end{array}$ & LmexTIM \\
\hline $\begin{array}{l}6 \beta-O-\text { Cinnamoyl-12-hydroxy-(13)15-en-16,12-olide- } \\
18 \text {-cassaneoic acid }\end{array}$ & -107.7 & -94.7 & -104.3 & -108.1 & -103.7 & -126.5 & -103.9 & -136.7 & -133.4 & -117.8 & -110.2 & -91.8 \\
\hline $6 \alpha, 7 \beta$-Diacetoxyvouacapane & -83.2 & -82.4 & -78.8 & -74.7 & -77.3 & -99.7 & -86.4 & -103.6 & -102.4 & -86.7 & -85.6 & -81.5 \\
\hline $\begin{array}{l}6 \beta-O-23^{\prime} \text {-Dihydrocinnamoyl-12-hydroxy-(13)15-en-16,12- } \\
\text { olide-18-cassaneoic acid }\end{array}$ & -108.6 & -100.4 & -52.6 & -101.3 & -97.7 & -125.8 & -92.8 & -140.1 & -130.8 & -118.5 & -109.0 & -102.6 \\
\hline \multicolumn{13}{|l|}{ Icetaxane diterpenoids } \\
\hline Cyclocoulterone & -89.0 & -82.5 & -71.2 & -81.3 & -78.1 & -117.3 & -70.7 & -87.7 & -98.4 & -87.3 & -83.5 & -79.3 \\
\hline 5-epi-Icetexone & -90.5 & -80.3 & -85.1 & -27.2 & -75.6 & -99.1 & -68.4 & -89.5 & -88.3 & -78.6 & -80.3 & -80.3 \\
\hline Komaroviquinone & -75.5 & -81.9 & -77.6 & -76.5 & -72.6 & -105.7 & -73.9 & -90.4 & -92.8 & -82.4 & -81.2 & -69.5 \\
\hline \multicolumn{13}{|l|}{ Mulinane diterpenoids } \\
\hline Azorellanol & -80.6 & -82.6 & -87.2 & -80.0 & -71.4 & -100.2 & -77.7 & -95.1 & -100.2 & -89.9 & -94.0 & -84.8 \\
\hline 7-Deacetylazorellanol & -73.7 & -90.1 & -81.0 & -72.4 & -75.4 & -103.4 & -72.2 & -91.8 & -88.7 & -82.8 & -89.1 & -72.3 \\
\hline Mulin-11,13-dien-20-oic acid & -84.7 & -95.2 & -85.0 & -71.5 & -71.3 & -97.5 & -72.5 & -98.2 & -99.2 & -80.6 & -92.2 & -86.8 \\
\hline Mulinic acid & -63.1 & -93.4 & -72.3 & -82.3 & -70.2 & -98.9 & -63.7 & -85.5 & -94.4 & -77.2 & -87.1 & -83.0 \\
\hline Mulinolic acid & -76.7 & -95.1 & -77.5 & -85.1 & -78.7 & -105.7 & -68.8 & -94.1 & -94.7 & -80.4 & -87.4 & -82.6 \\
\hline \multicolumn{13}{|l|}{ Miscellaneous diterpenoids } \\
\hline Hypoestoxide & -80.6 & -92.1 & -67.1 & -85.2 & -78.1 & -94.9 & -85.7 & -114.8 & -102.4 & -79.6 & -87.9 & -72.7 \\
\hline Komarovispirone & -77.7 & -80.5 & -67.4 & -75.5 & -70.3 & -101.0 & -77.5 & -90.5 & -92.3 & -86.1 & -80.8 & -79.0 \\
\hline Sacculatal & -86.4 & -84.1 & -84.0 & -82.4 & -77.2 & -103.5 & -78.1 & -92.5 & -104.3 & -87.9 & -98.7 & -79.1 \\
\hline Serratol & -67.7 & -78.7 & -59.5 & -77.4 & -67.0 & -89.9 & -71.4 & -94.3 & -92.3 & -82.9 & -90.1 & -70.1 \\
\hline Totarol & -63.9 & -70.3 & -59.4 & -69.8 & -60.5 & -88.0 & -68.6 & -84.0 & -81.5 & -72.2 & -90.4 & -65.7 \\
\hline
\end{tabular}


Table 28. MolDock docking energies $(\mathrm{kJ} / \mathrm{mol})$ of miscellaneous diterpenoids with Leishmania infantum protein targets.

\begin{tabular}{|c|c|c|c|c|c|}
\hline Kaurane diterpenoids & LinfCYP51 & LinfGLO2 & LinfPnC1 & LinfTDR1 & LinfTR \\
\hline 15-Angeloyl-4 $\alpha, 15 \beta$-kaur-16-en-18-oic acid & -97.4 & -64.6 & no dock & -82.4 & -95.6 \\
\hline ent-11 $\alpha$-Hydroxy-16-kauren-15-one & -82.8 & -66.5 & no dock & -66.5 & -76.6 \\
\hline Kaurenoic acid & -79.9 & -65.8 & no dock & -77.4 & -82.6 \\
\hline Perymenic acid & -98.3 & -77.0 & no dock & -83.2 & -97.0 \\
\hline ent-15ß-Senecioyloxy-16,17-epoxy-kauran-18-oic acid & -99.6 & -77.9 & no dock & -85.4 & -102.2 \\
\hline \multicolumn{6}{|l|}{ Pimarane diterpenoids } \\
\hline Acanthoic_acid & -83.6 & -64.6 & no dock & -72.4 & -82.6 \\
\hline 7 $\beta$-Hydroxy-ent-pimara-8(14),15-dien-19-oic acid & -90.4 & -67.2 & no dock & -75.0 & -79.2 \\
\hline ent-15-Pimarene-8 $\beta, 19-$ diol & -84.0 & -63.8 & no dock & -69.9 & -77.4 \\
\hline ent-8(14),15-Pimaradien-3 $\beta$-acetoxy & -90.6 & -73.3 & no dock & -70.5 & -92.1 \\
\hline ent-8(14),15-Pimaradien-3 $\beta, 19-$ diol & -82.2 & -69.2 & no dock & -67.0 & -78.9 \\
\hline ent-Pimara-8(14),15-dien-19-oic acid & -84.2 & -69.0 & no dock & -70.8 & -80.3 \\
\hline ent-8(14),15-Pimaradien-3b-ol & -78.3 & -67.2 & no dock & -66.8 & -79.1 \\
\hline \multicolumn{6}{|l|}{ Cassane diterpenoids } \\
\hline $\begin{array}{l}6 \beta-O-\text { Cinnamoyl-12-hydroxy-(13)15-en-16,12-olide-18-cassaneoic } \\
\text { acid }\end{array}$ & -125.4 & -91.1 & no dock & -105.3 & -119.8 \\
\hline $6 \alpha, 7 \beta-$ Diacetoxyvouacapane & -93.8 & -77.7 & no dock & -83.7 & -93.9 \\
\hline $\begin{array}{l}6 \beta-O-23^{\prime}-\text { Dihydrocinnamoyl-12-hydroxy-(13)15-en-16,12-olide-18-cassaneoic } \\
\text { acid }\end{array}$ & -124.7 & -88.7 & no dock & -99.0 & -114.1 \\
\hline \multicolumn{6}{|l|}{ Icetaxane diterpenoids } \\
\hline Cyclocoulterone & -90.3 & -68.7 & no dock & -76.9 & -90.0 \\
\hline 5-epi-Icetexone & -99.1 & -75.8 & no dock & -81.0 & -81.6 \\
\hline Komaroviquinone & -96.8 & -72.4 & no dock & -75.6 & -88.7 \\
\hline \multicolumn{6}{|l|}{ Mulinane diterpenoids } \\
\hline Azorellanol & -99.1 & -72.4 & no dock & -78.2 & -91.2 \\
\hline 7-Deacetylazorellanol & -88.5 & -71.4 & no dock & -76.5 & -85.9 \\
\hline Mulin-11,13-dien-20-oic acid & -87.5 & -72.9 & no dock & -79.1 & -78.6 \\
\hline Mulinic acid & -88.3 & -70.6 & no dock & -73.8 & -84.3 \\
\hline Mulinolic acid & -89.0 & -78.1 & no dock & -78.6 & -85.8 \\
\hline \multicolumn{6}{|l|}{ Miscellaneous diterpenoids } \\
\hline Hypoestoxide & -97.2 & -82.2 & no dock & -73.6 & -91.2 \\
\hline Komarovispirone & -83.1 & -72.0 & no dock & -74.4 & -79.9 \\
\hline Sacculatal & -93.5 & -77.7 & no dock & -84.4 & -88.1 \\
\hline Serratol & -81.7 & -77.1 & no dock & -78.5 & -78.5 \\
\hline Totarol & -76.4 & -67.7 & no dock & -67.9 & -73.4 \\
\hline
\end{tabular}

Over 100 antiparasitic diterpenoids were docked into the protein targets in this study. These include abietane-, clerodane-, kaurane-, labdane-, pimarane-, cassane- and mulinane-like diterpenoids. In general, diterpenoids preferentially dock to LmajMetRS, LmajDHODH and LmexGPDH. 12-Odeacetyl-6-O-acetyl-18-acetyloxycoleon $\mathrm{Q}$, the antiplasmodial diterpene isolated from the tropical Asia plant Anisochilus harmandii (Lamiaceae) [64] has a stronger docking energy $(-111.8 \mathrm{~kJ} / \mathrm{mol}$ ) for LmajPDE1 than the co-crystallized competitive phosphodiesterase inhibitor, 3-isobutyl-1- 
methylxanthine (docking energy $=-78.1 \mathrm{~kJ} / \mathrm{mol}$ ). 14(R)-Aulacocarpin $\mathrm{C}$ and $15(S)$-methoxy-labdan8(17),11(E),13(14)-trien-15,16-olide are also selective for LmexPYK and LmajOPB, respectively. The cassane-like antileishmanial diterpenes 6 6-O-2'3'-dihydrocinnamoyl-12-hydroxy-(13)15-en-16,12olide-18-cassaneoic acid and 6 $\beta$-O-cinnamoyl-12-hydroxy-(13)15-en-16,12-olide-18-cassaneoic acid have stronger docking energies than any of the other docked diterpenoids for most of the protein targets. These higher docking energies correlate with the fact they have higher molecular weights that the other diterpenoids. Despite the molecular weight correlation, 6 $\beta-O-2$ '3'-dihydrocinnamoyl-12hydroxy-(13)15-en-16,12-olide-18-cassaneoic acid (MW: 496.592) is selective for LmajNMT. The compound's docking score for LmajNMT is comparable to that of the protein's co-crystallized pyrazole sulfonamide inhibitor (PDB ID: 4a30; MW: 495.425; docking energy $=-121.1 \mathrm{~kJ} / \mathrm{mol}$ ). The lowest energy pose of 63-O-2'3'-dihydrocinnamoyl-12-hydroxy-(13)15-en-16,12-olide-18-cassaneoic acid is predicted to have extensive interactions with residues Ala 204, Asp 83, Asp 84, Glu 82, Gly 205, Phe 88, Phe 90, Tyr 217, Tyr 345, Val 81 and Val 206. The cassane diterpenoid is predicted to have hydrogen bonding interactions through its carboxylic acid moiety and the ester bond of its cinnamoyl substituent to the backbone carbonyl group of Gly 205, and the side chain phenolic residue of Tyr 217, respectively (Figure 19). The icetaxane diterpenoids 5-epi-icetaxone showed preferential docking to L. infantum sterol $14 \alpha$-demethylase (LinfCYP51) with a lower docking energy than the co-crystallized ligand, fluconazole $(-99.1$ and $-91.3 \mathrm{~kJ} / \mathrm{mol}$, respectively).

Figure 19. The lowest-energy pose of $6 \beta-O-23^{\prime}$ '-dihydrocinnamoyl-12-hydroxy-(13)15-en16,12-olide-18-cassaneoic acid and LmajNMT. The hydrogen bonding interactions between the ligand and the protein (Gly 205 and Tyr 217) is shown as blue dash lines. Val 81, Asp 83 and Phe 88 were also predicted to have very strong steric interactions with the ligand.

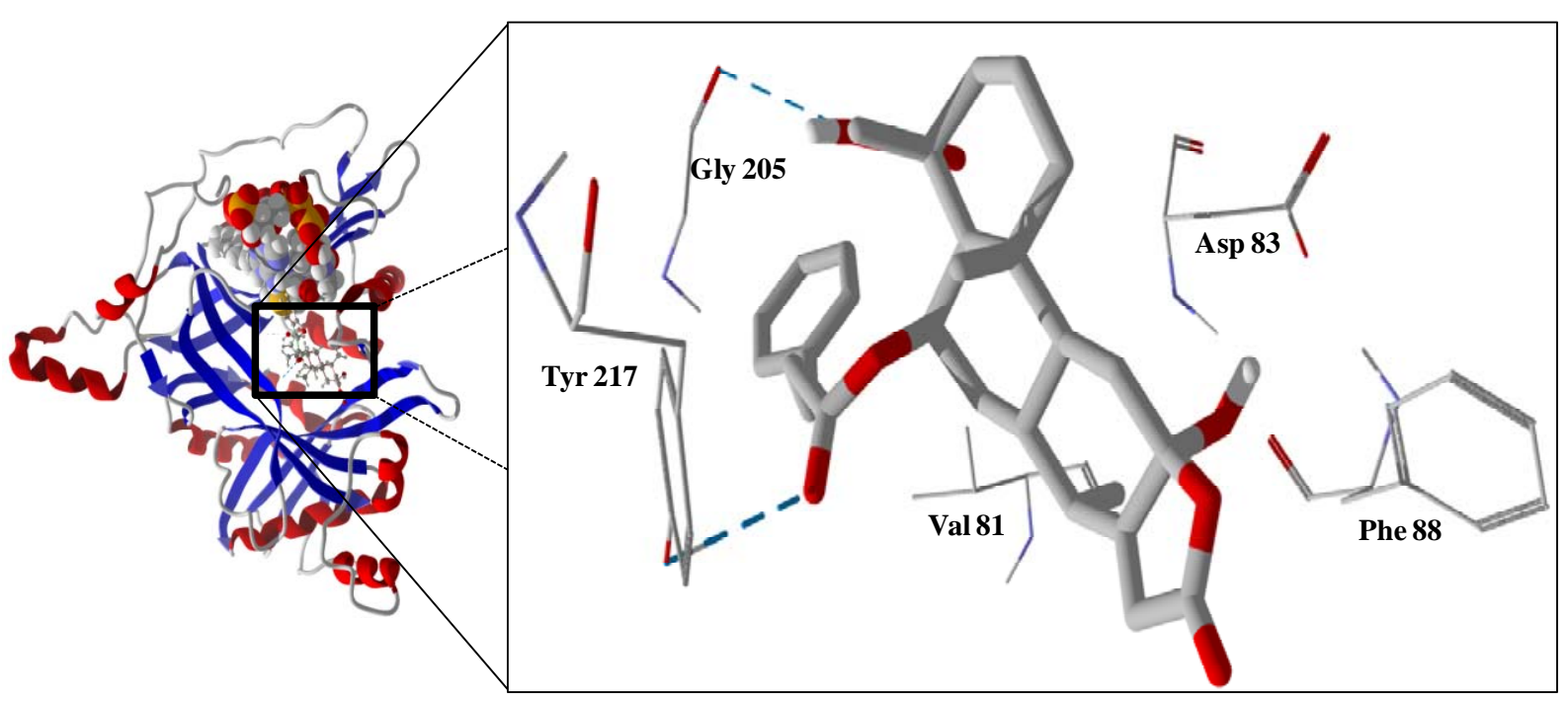

\subsection{Triterpenoid Docking}

Triterpenoids, including limonoids, withanolides, quassinoids, and steroids are shown in Figures 20-24. The docking energies for the triterpenoid-based ligands are compiled in Tables 29-40. The triterpenoids ligands with the strongest docking energies were the limonoids carapolide A and 
khayanolide A, and the withanolides 24,25-epoxywithanolide D and withangulatin A. Carapolide A showed significant docking to LmajDHODH (docking energy $=-140.0 \mathrm{~kJ} / \mathrm{mol}$ ). Khayanolide A preferentially docked with LmajMetRS and LmexGPDH (docking energies $=-138.5$ and $-137.8 \mathrm{~kJ} / \mathrm{mol}$, respectively). The limonoid with the strongest docking was, however, grandifotane with LmajDHODH with a docking energy of $-154.4 \mathrm{~kJ} / \mathrm{mol}$. 6-O-Acetylswietenolide docked strongly to LmexGPDH (docking energy $=-142.7 \mathrm{~kJ} / \mathrm{mol}$ ). The withanolide with the strongest docking was physagulin $\mathrm{F}$ with LmajMetRS, which had a docking energy of $-133.2 \mathrm{~kJ} / \mathrm{mol}$. As a class, the steroids showed the most target selectivity with six of the seven steroids significantly docking more strongly to LmajMetRS. In general, limonoids showed some selectivity for LmexGPDH and LmajDHODH, while withanolides docked more selectively with LmajUGPase.

Figure 20. Triterpenoids examined in this work.<smiles>C[C@H]1CCC2(C)CC[C@]3(C)C(=CCC4C5(C)CCC(O)C(C)(C)C5CCC43C)C2[C@@H]1C</smiles>

$\alpha$-Amyrin<smiles>C=C(C)[C@H]1CCC2(C)CC[C@]3(C)C(CCC4C5(C)CCC(O)C(C)(C)C5CCC43C)C12</smiles>

Betulinaldehyde<smiles>CC1(C)CCC2(CO)CC[C@]3(C)C(=CCC4C5(C)CCC(O)C(C)(C)C5CCC43C)C2C1</smiles>

Erythrodiol<smiles>CC1(C)CCC2(C)CC[C@]3(C)C(=CCC4C5(C)CCC(O)C(C)(C)C5CCC43C)C2C1</smiles>

$\beta$-Amyrin<smiles>C=C(C)[C@H]1CCC2(C(=O)O)CC[C@]3(C)C4CCC5C(C)(C)C(O)CCC5(C)C4CCC3[C@]12C</smiles>

Betulinic acid<smiles>CC1C(=O)CCC2C1(C)CCC1(C)C2(C)CCC2C1(C)CCC1(C)C2(C)CCC2(C)CCC(C)(C)CC21C</smiles>

Friedelin<smiles>C=C(C)[C@@H]1CCC2(CO)CC[C@]3(C)C4CCC5C(C)(C)C(O)CCC5(C)C4CCC3(C)C12</smiles>

Betulin

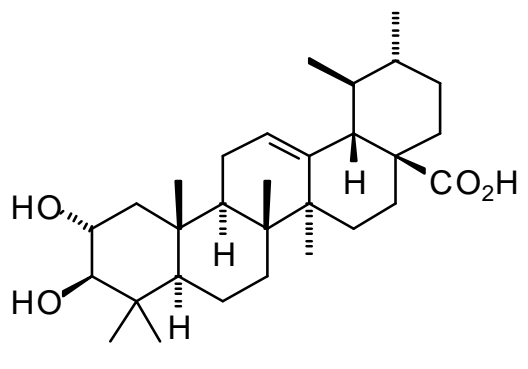

Corosolic acid<smiles>C=C1CCC2(C)CCC3(C)C4CC=C5C(=CC(=O)C(O)=C5C)C45CCC[C@]3(C2)[C@@H]1C5</smiles>

Isoiguesterin 
Figure 20. Cont.<smiles>CC1=C(O)C(=O)C=C2C1=CC=C1C23CC[C@]2(C)C4(C)CCC(CO)CC4(C)CCC2(C)C13C</smiles>

20-epi-Isoiguesterinol

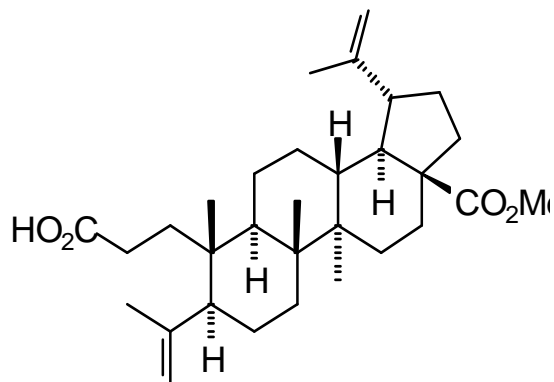

Methyl seco-3,4-betulonic acid<smiles>CC1(C)CCC2(C(=O)O)CC[C@]3(C)C(=CCC4C5(C)CC[C@H](O)C(C)(C)C5CCC43C)C2C1</smiles>

epi-Oleanolic acid<smiles>CC(=O)C1(C)CCC2(C)CCC3(C)C4=CC=C5C(=CC(=O)C(O)=C5C)C45CCC(C3C2)[C@@H]5C1</smiles>

Pristimerin

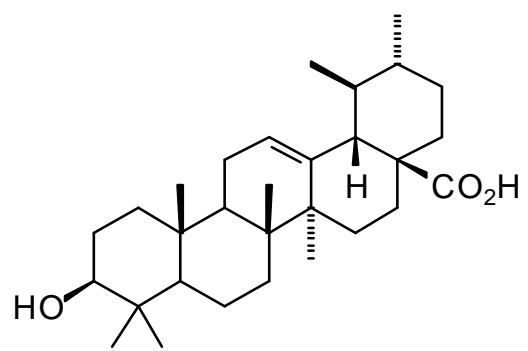

Ursolic acid<smiles>C=C(C)[C@@H]1CCC2(C)CC[C@]3(C)C4CCC5C(C)(C)C(O)CCC5(C)C4CCC3(C)C12</smiles>

Lupeol<smiles>COc1c(O)cc2c(c1C)C(=O)C=C1C2(C)CC[C@]2(C)C3CC(C)(C(N)=O)CCC3(C)CCC12C</smiles>

3-0-Methyl-6-oxopristimerol<smiles>CC(=O)C1(C)CCC2(C)CCC3(C)C4=CC(=O)c5c(cc(O)c(O)c5C)C4(C)CC[C@]3(C)[C@H]2C1</smiles>

6-Oxopristimerol

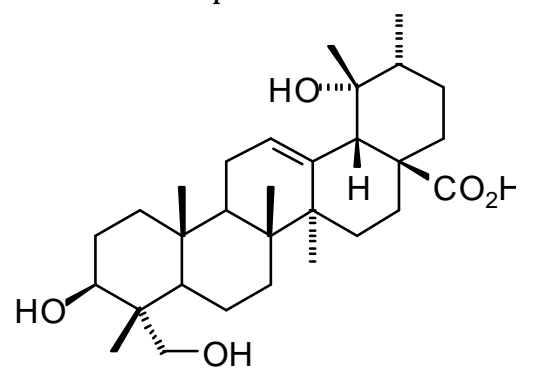

Rotundic acid<smiles>CC1CCC2(CO)CC[C@]3(C)C(=CCC4C5(C)CCC(O)C(C)(C)C5CCC43C)C2C1C</smiles>

Uvaol

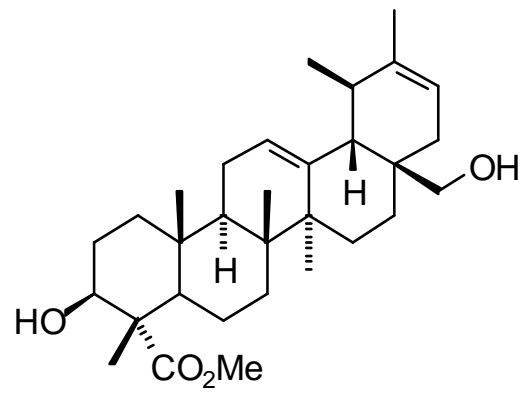

Lawnermis acid methyl ester<smiles>CC1(C)CCC2(C(=O)O)CC[C@]3(C)C(=CCC4C5(C)CCC(O)C(C)(C)C5CCC43C)C2C1</smiles>

Oleanolic acid<smiles>C/C(=C\CC[C@@H](C)[C@H]1CCC2(C)C3=CCC4C(C)(C)C(=O)CCC4(C)[C@]3(C)CC[C@]12C)C(=O)O</smiles>

(24Z)-3-0xotirucalla-7,24-dien-

26-oic acid<smiles>CC1(C)CCC2(C)CC=C3C4(C)CCC5C(C)(C)CCC(O)C5(C)C4CC[C@]3(C)C2C1</smiles>

Taraxerol<smiles>CC(CO)[C@H]1CCC2(C)CC[C@]3(C)C4CCC5C(C)(C)C(O)CCC5(C)C4CCC3(C)C12</smiles>

Wallichianol 
Figure 21. Steroids examined in this work.<smiles>CC(C)CCC[C@H](C)C1CCC2C1(C)CCC1C3(C)CCC(O)CC3=CCC12C</smiles>

Cholesterol<smiles>CC(C)=CCC[C@@H](C)C1CC[C@]2(C)C3=C(CC[C@]12C)[C@@]1(C)CCC(O)C(C)(C)[C@@]1(C)CC3</smiles>

Lanosterol<smiles>CCC(/C=C/[C@H](C)C1CCC2C3CC=C4CC(O)CCC4(C)C3CCC21C)C(C)C</smiles><smiles>C=C(C)[C@H](CC)CC[C@H](C)C1CCC2C3CC=C4CC(O)CCC4(C)C3CCC21C</smiles>

Clerosterol<smiles>C=C[C@](O)(CC[C@H](C)C1CCC2C3CC=C4CC(O)CCC4(C)C3CCC21C)C(C)C</smiles>

Seringosterol<smiles>C=C[C@](O)(CC[C@H](C)C1CCC2C3CC=C4CC(O)CCC4(C)C3CCC21C)C(C)C</smiles>

24-Hydroperoxy-24-vinylcholesterol<smiles>CCC(CC[C@H](C)C1CCC2C3CC=C4CC(O)CCC4(C)C3CCC21C)C(C)C</smiles>

$\beta$-Sitosterol

Stigmasterol

Figure 22. Quassinoids examined in this work.

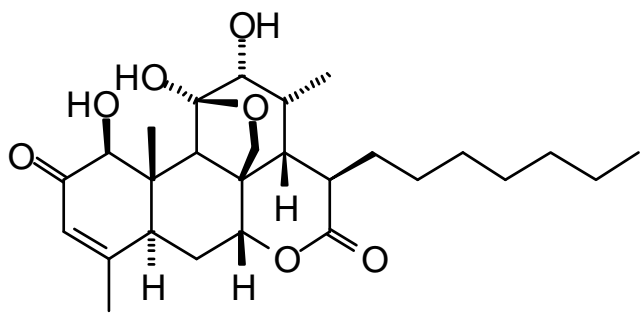

15- $\beta$-Heptylchaparrinone

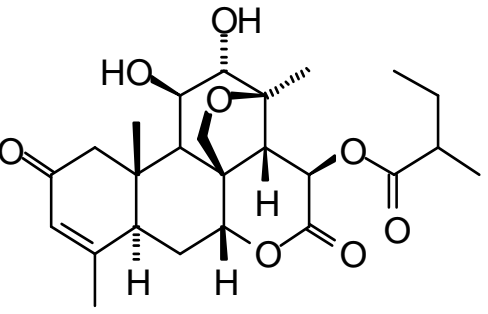

Simalikalactone D

Figure 23. Limonoids examined in this work.

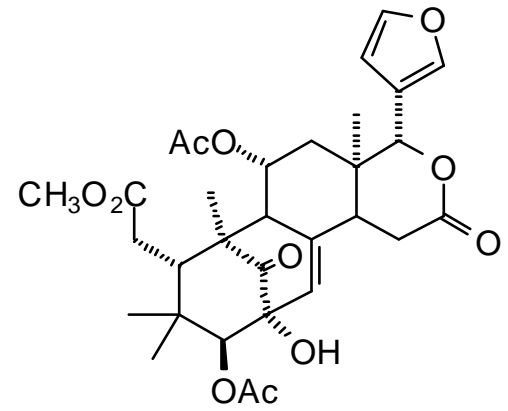

$11 \alpha$-Acetoxy-2 $\alpha$-hydroxy-6deoxyswietenine acetate

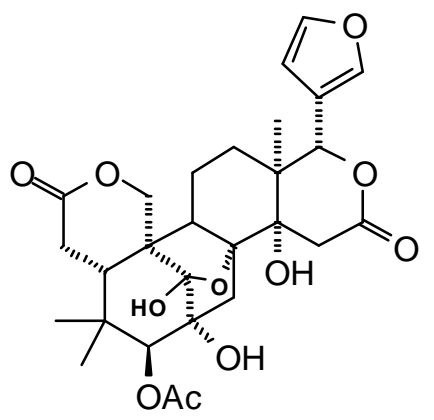

3-O-Acetylanthothecanolide

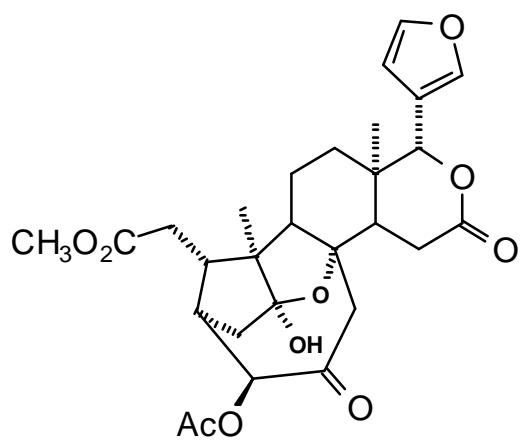

3-O-Acetylkhayalactone 
Figure 23. Cont.

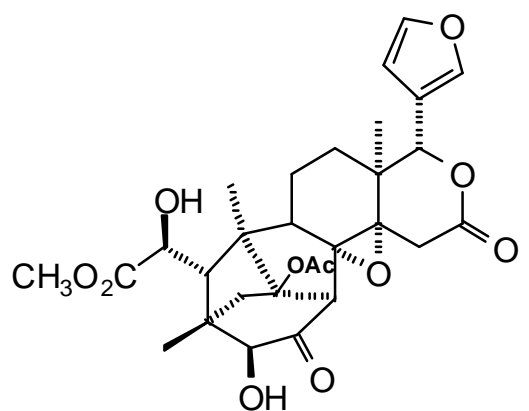

1-O-Acetylkhayanolide A

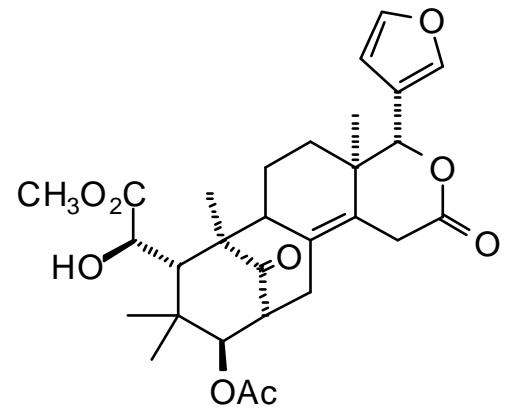

3-O-Acetylswietenolide

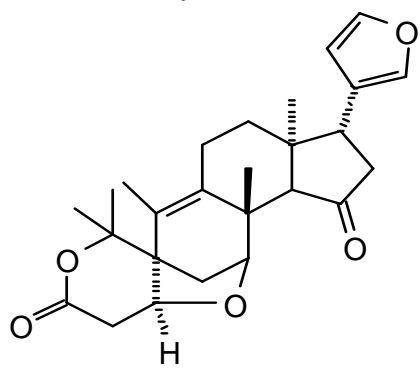

Carapa spirolactone

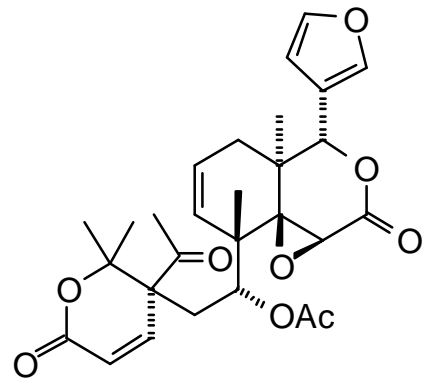

Carapolide B

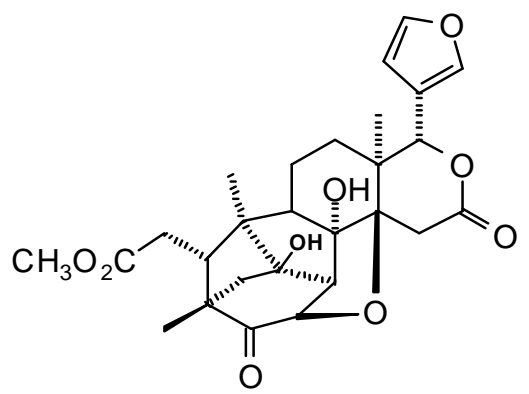

1-Deacetyl-6-deoxykhayanolide E

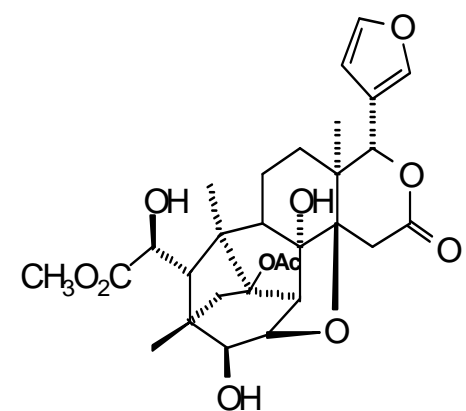

1-O-Acetylkhayanolide B

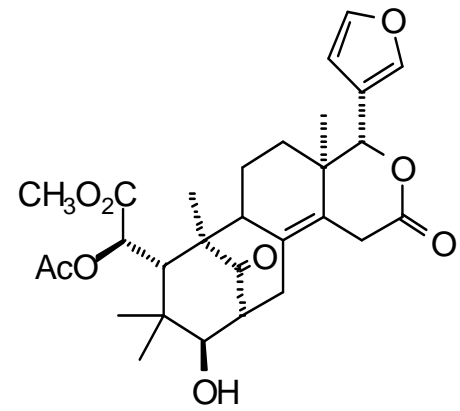

6-O-Acetylswietenolide

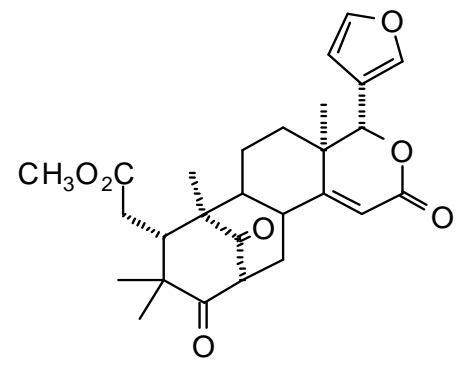

Carapin

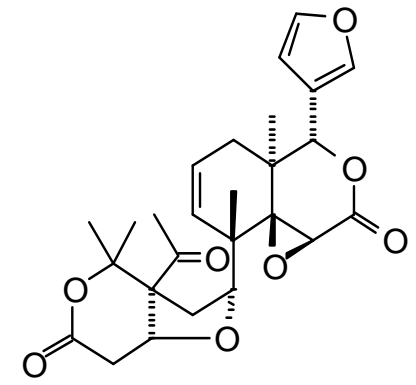

Carapolide C<smiles>CC1(C)C(=O)C=CC2(C)C1C[C@H](O)C1(C)C2CC[C@]2(C)C(c3ccoc3)O[C@H](O)C3OC312</smiles>

7-Deacetylgedunin<smiles>CO[C@H](O)[C@H]1[C@H](O)[C@@]2(C)C3CC[C@@]4(C)C(CC(=O)O[C@@H]4c4ccoc4)C3=C[C@@H]2[C@@H](OC(C)=O)C1(C)C</smiles>

3-O-Acetylswietenine

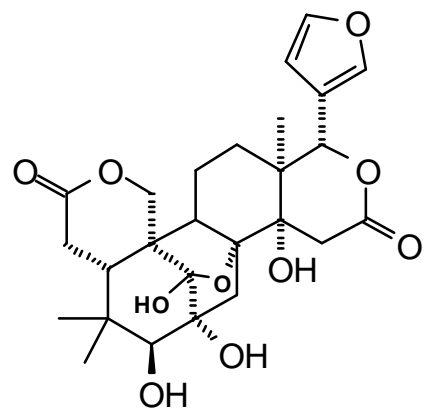

Anthothecanolide<smiles>CC(=O)O[C@H](CC1=CCC(=O)OC1(C)C)[C@@]1(C)C=CC[C@]2(C)[C@@H](c3ccoc3)OC(=O)C3OC321</smiles>

Carapolide A

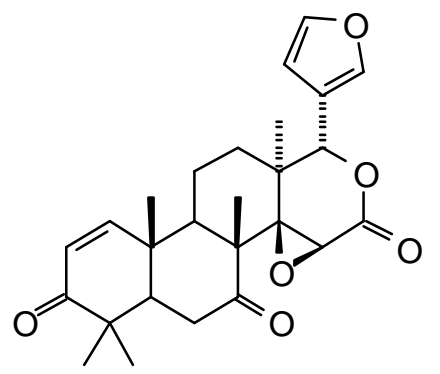

7-Deacetoxy-7-oxogedunin

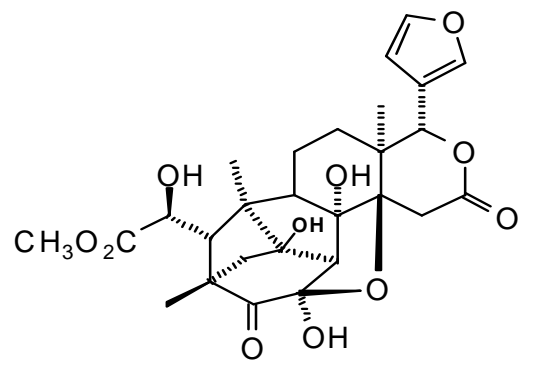

1-O-Deacetyl-2 $\alpha$ -

hydroxykhayanolide $\mathrm{E}$ 
Figure 23. Cont.

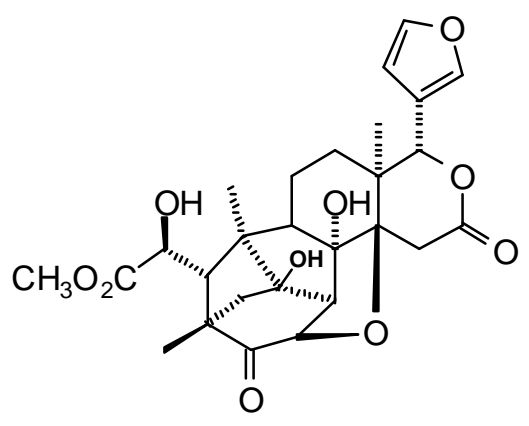

Deacetylkhayanolide E

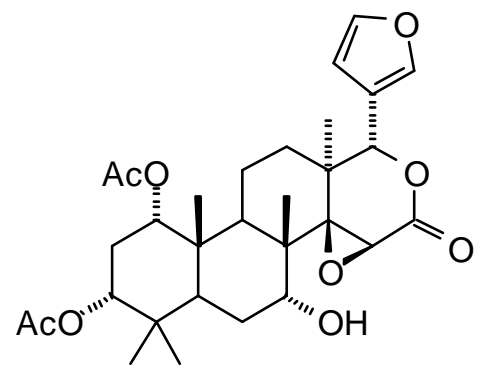

7-Deacetylkhivorin

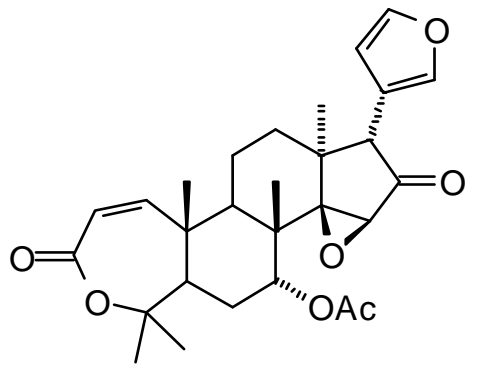

Evodulone

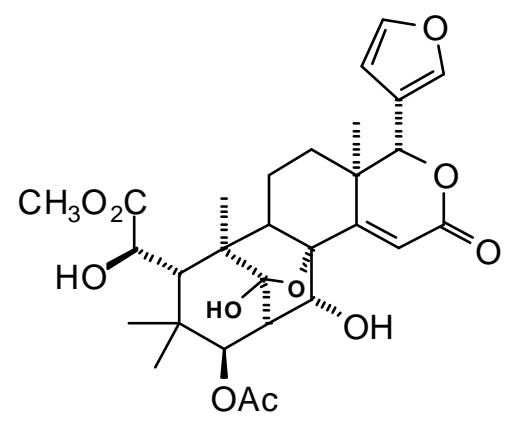

Grandifolide A

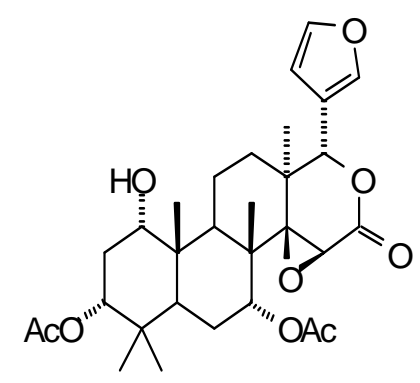

1-Deacetylkhivorin

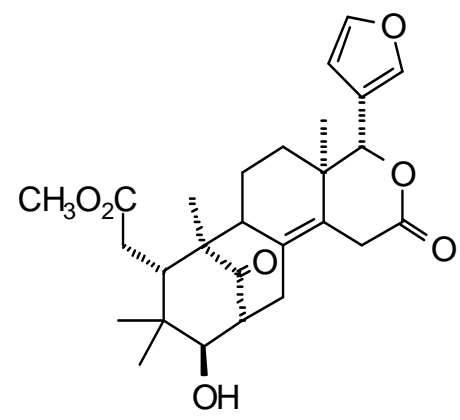

6-Deoxyswietenolide

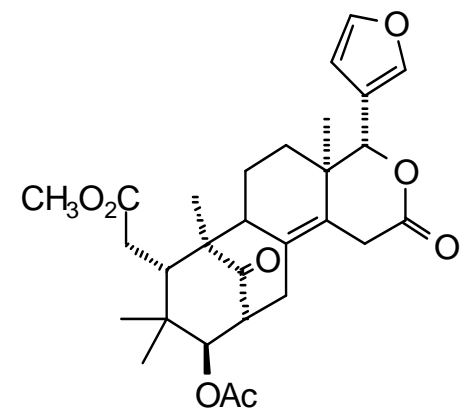

Fissinolide

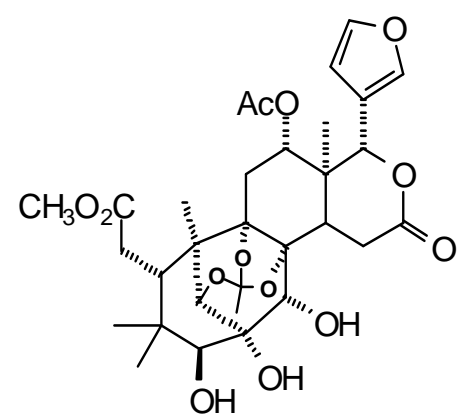

Grandifolin

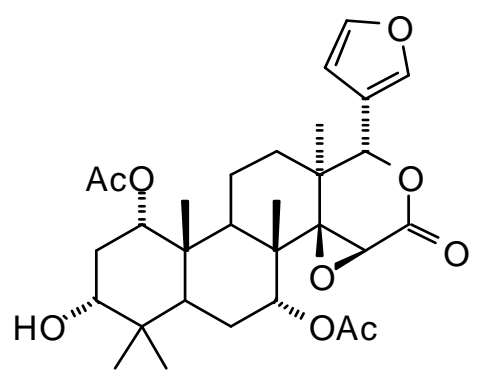

3-Deacetylkhivorin

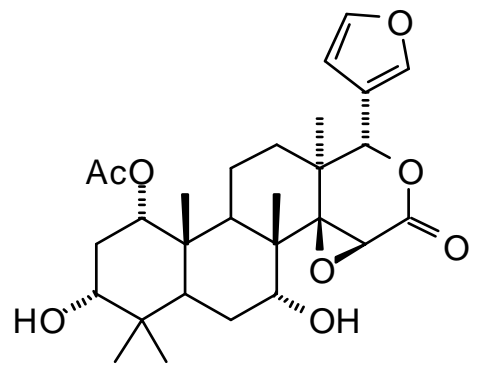

3,7-Dideacetylkhivorin

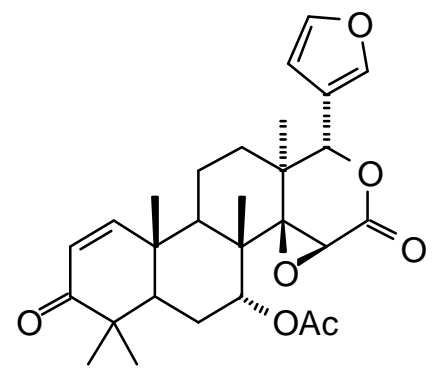

Gedunin<smiles>CC(=O)O[C@H]1CC2C(C)(C)C(=O)C=CC2(C)C2CC[C@]3(C)C(=CC[C@H]3C3COC(C(C)(C)O)[C@@H](O)C3)C21C</smiles>

Grandifoliolenone 
Figure 23. Cont.

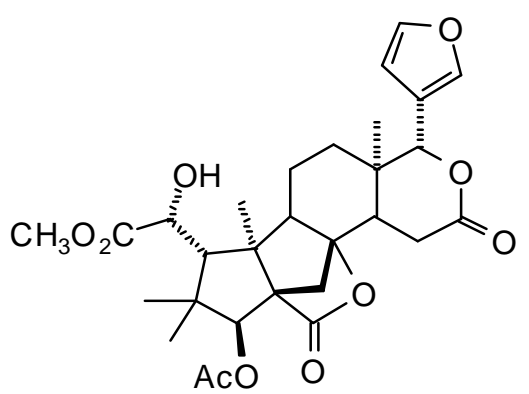

Grandifotane

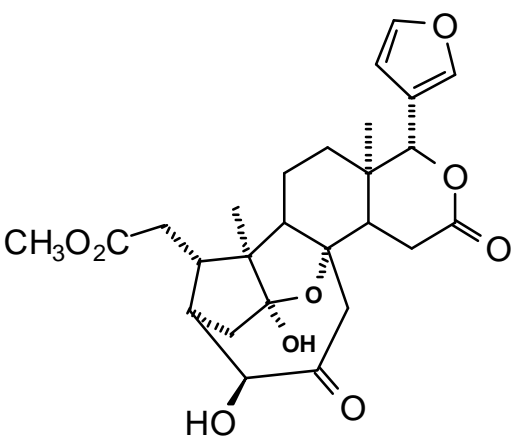

Khayalactone

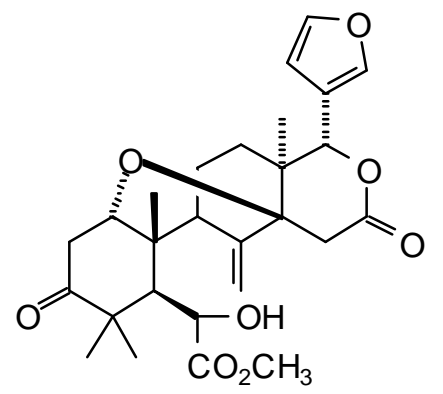

Methyl 6-hydroxyangolensate

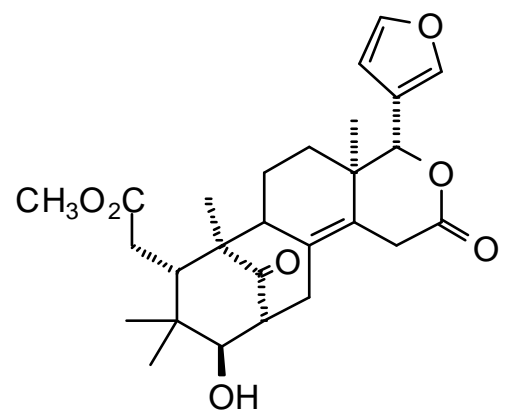

Proceranolide

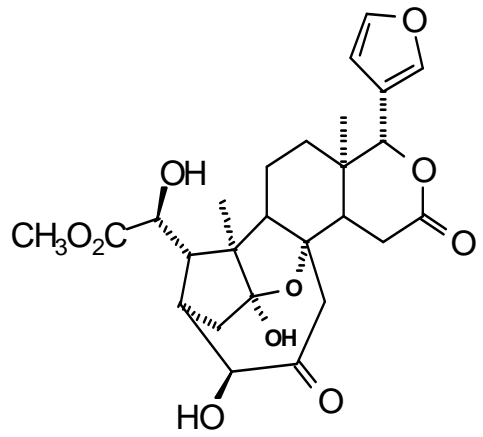

6-Hydroxykhayalactone

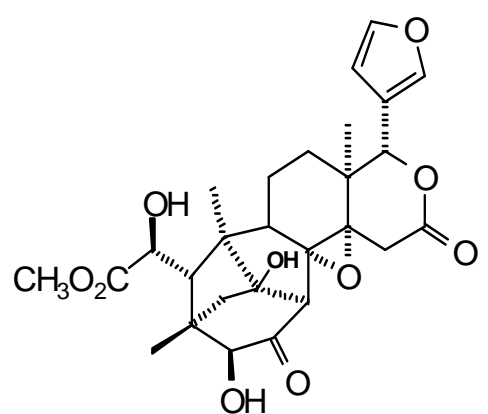

Khayanolide A

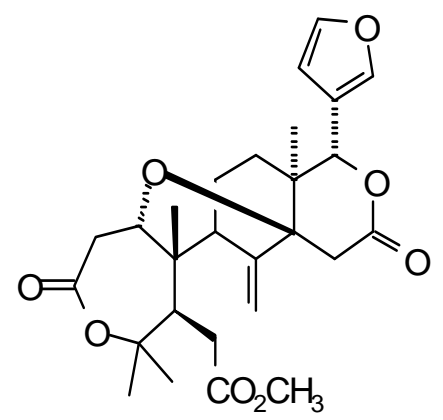

Methyl ivorensate

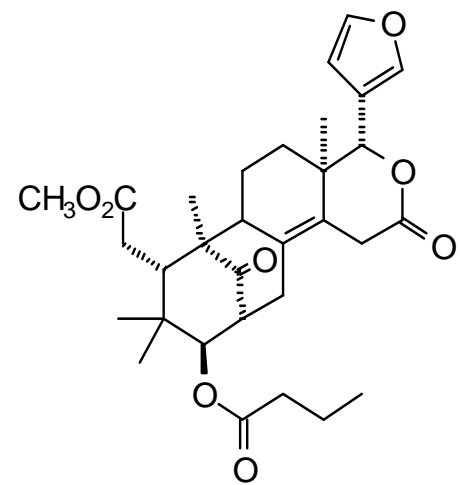

Proceranolide butanoate

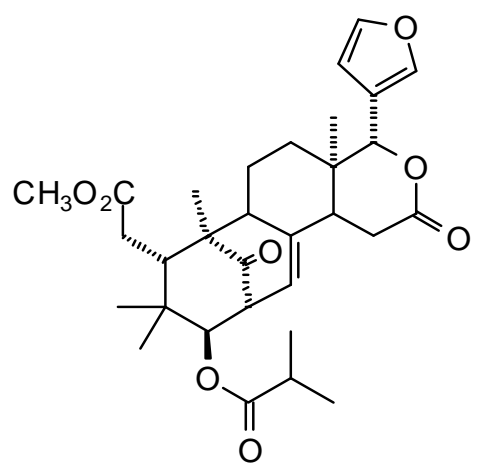

3ß-Isobutyryloxy-1-oxomeliac-8(30)enate

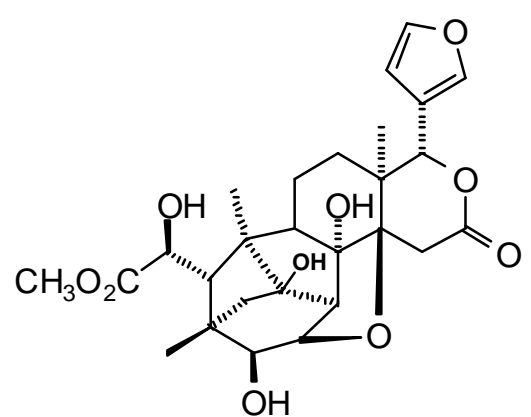

Khayanolide B

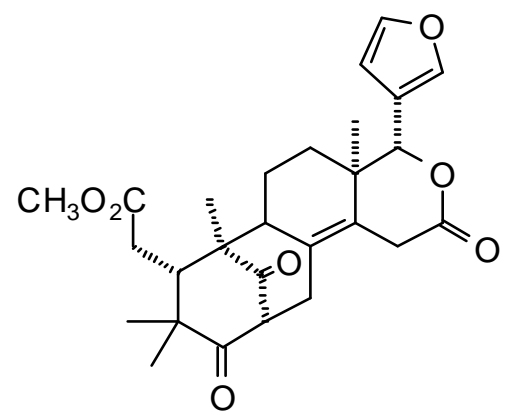

Mexicanolide<smiles>CC(=O)O[C@H]1CC2C(C)(C)OC(=O)C=CC2(C)C2CC[C@]3(C)C(=CC(=O)[C@H]3c3ccoc3)[C@@]21C</smiles>

Proceranone 
Figure 23. Cont.

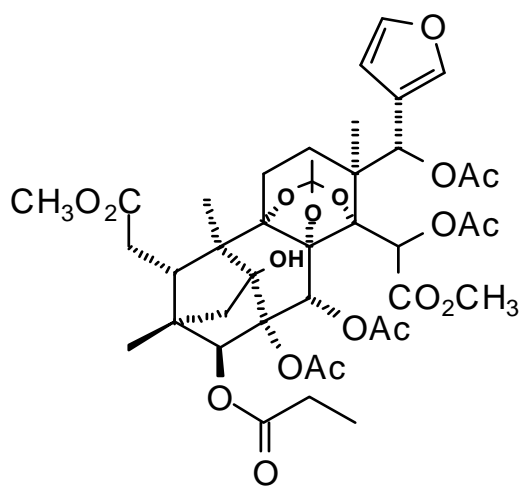

Procerin

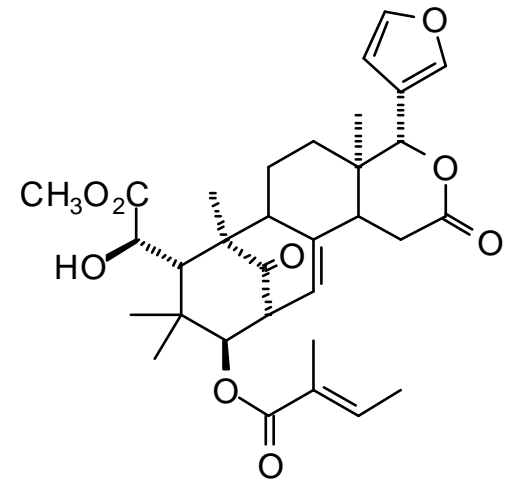

Swietenine

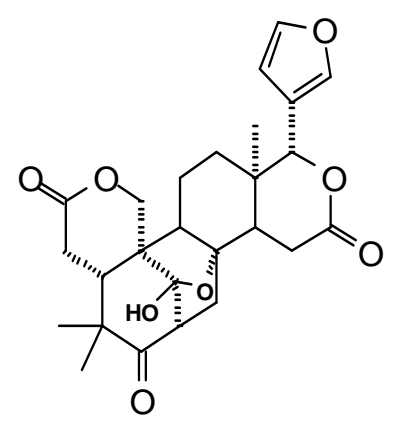

Seneganolide

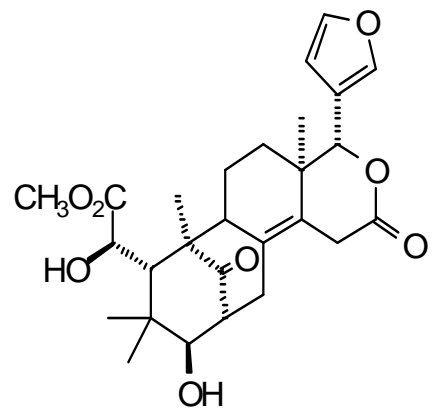

Swietenolide<smiles>COC(=O)CC1C(C)(C)C(=O)C=CC1(C)[C@H]1CC[C@@](C)([C@H](O)c2ccoc2)[C@@]2(O)CC(=O)OC12C</smiles>

Swiemahogin A

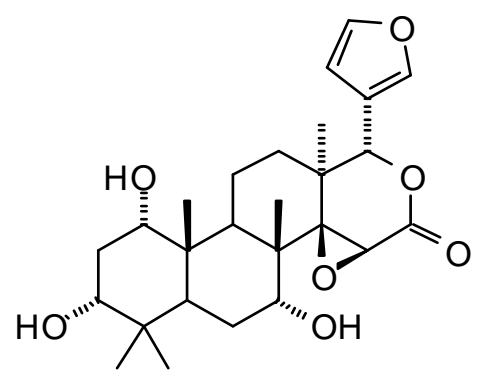

1,3,7-Trideacetylkhivorin

Figure 24. Withanolides examined in this work.

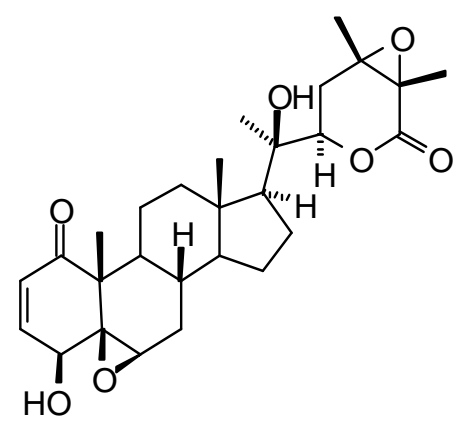

24,25-Epoxywithanolide D

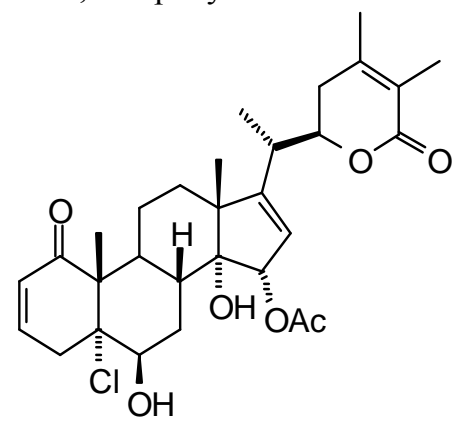

Physagulin B

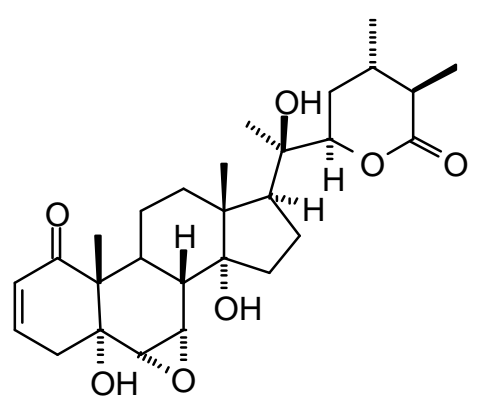

14-Hydroxyixocarpanolide

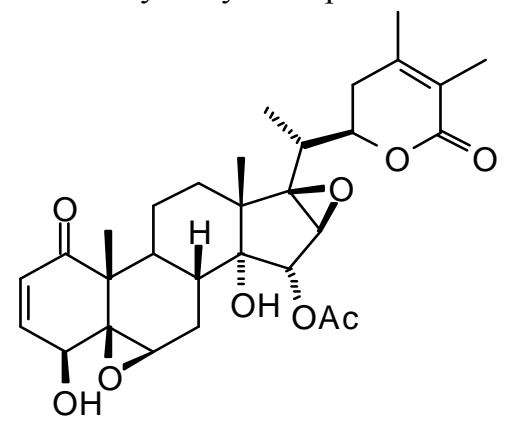

Physagulin C

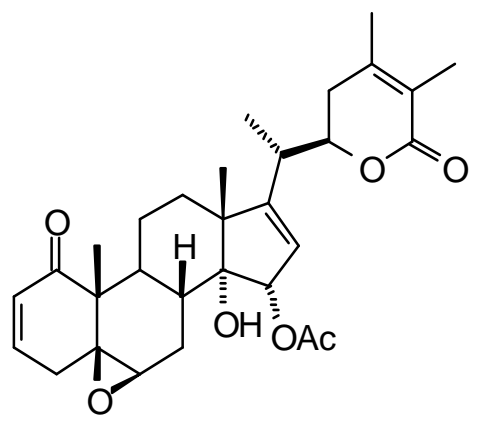

Physagulin A

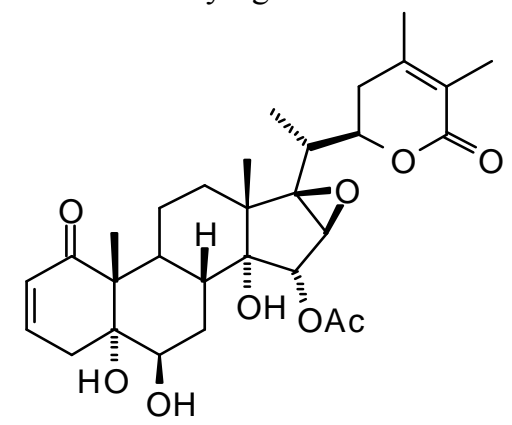

Physagulin F 
Figure 24. Cont.

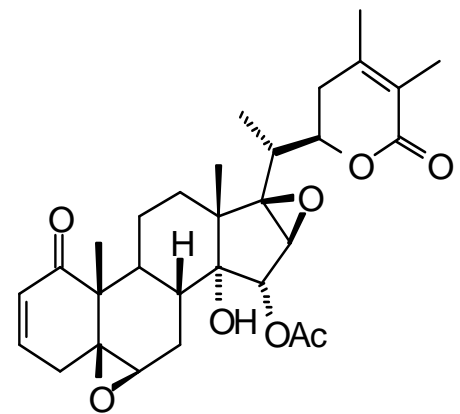

Physagulin H

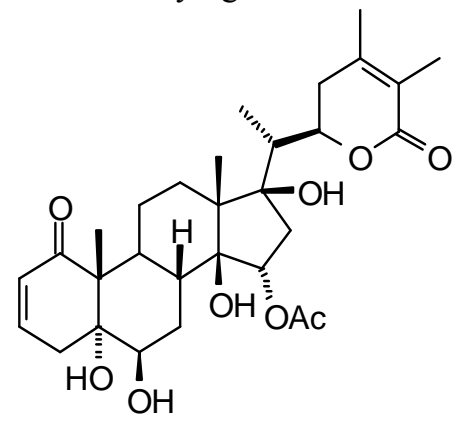

Physagulin K

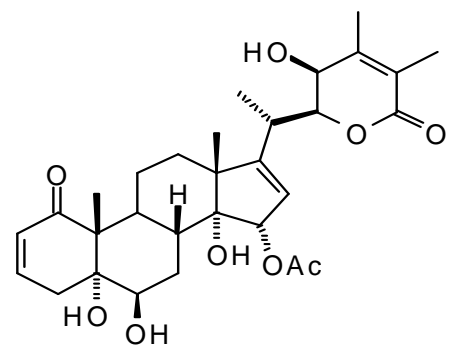

Physagulin M

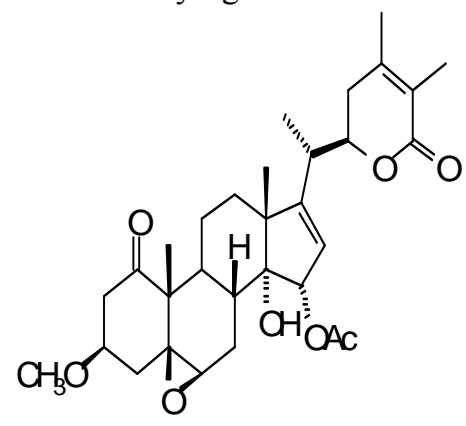

Physagulin $\mathrm{N}^{\prime}$

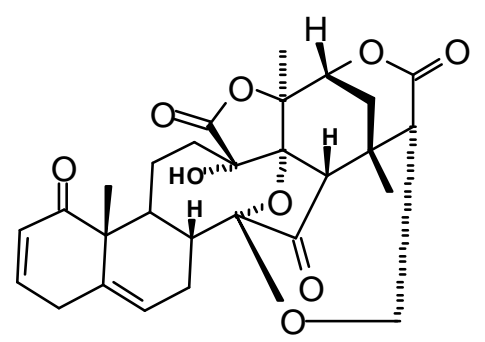

Physalin B

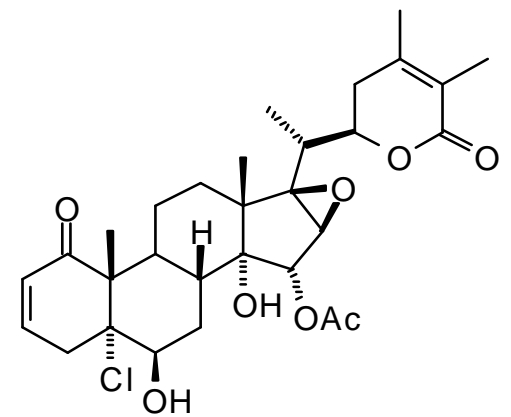

Physagulin I

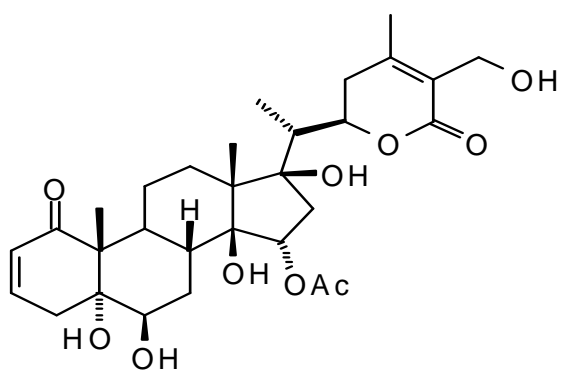

Physagulin L

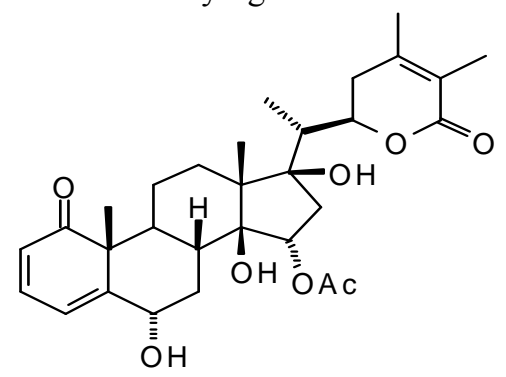

Physagulin $\mathrm{M}^{\prime}$

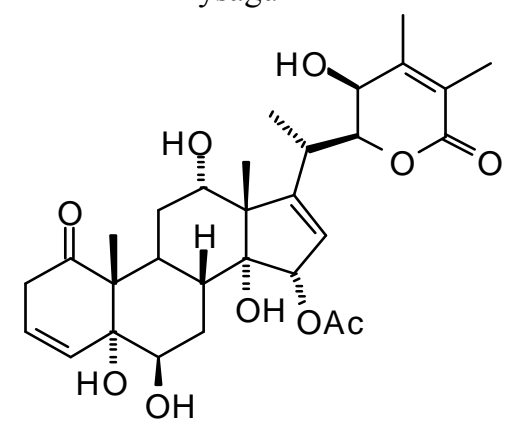

Physagulin O

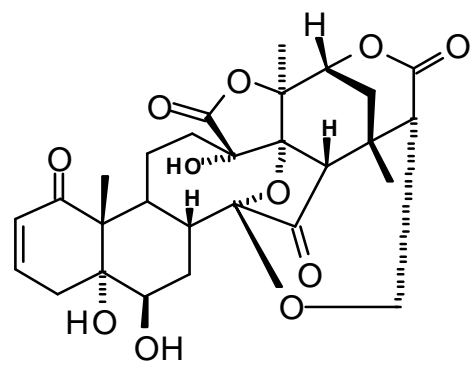

Physalin D

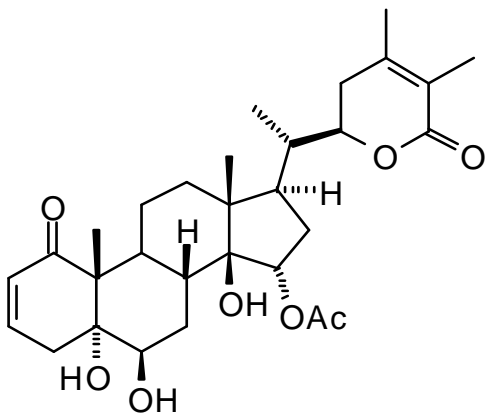

Physagulin J

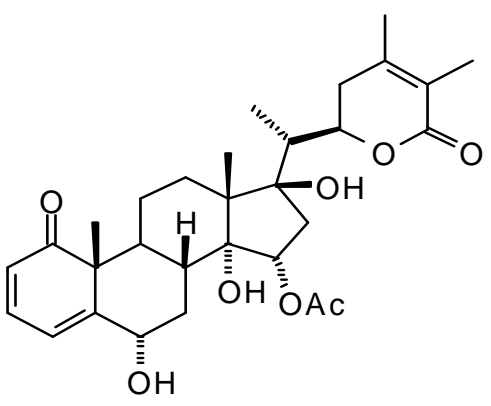

Physagulin L'

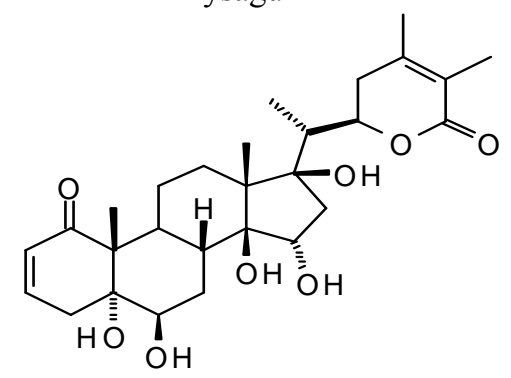

Physagulin N

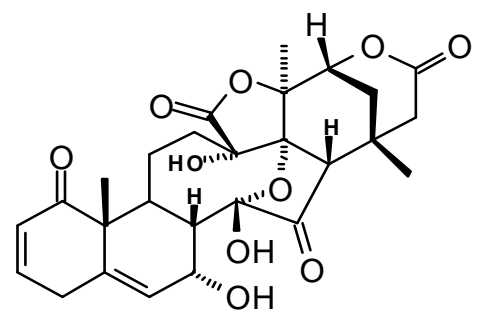

Physalin A

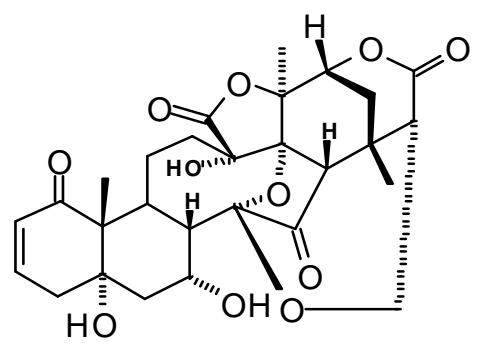

Physalin E 
Figure 24. Cont.

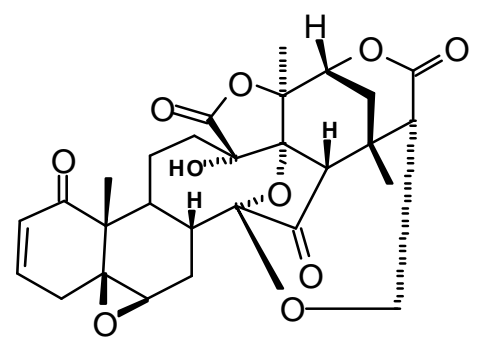

Physalin F

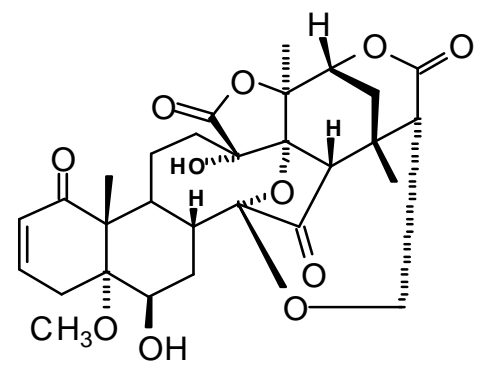

Physalin I

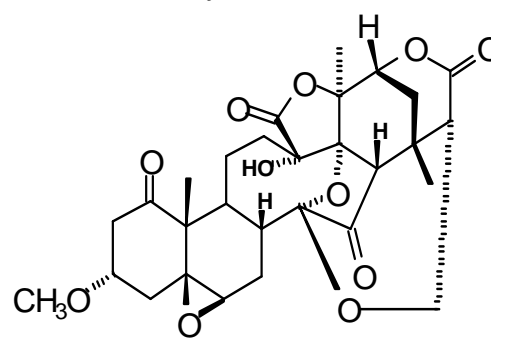

Physalin U

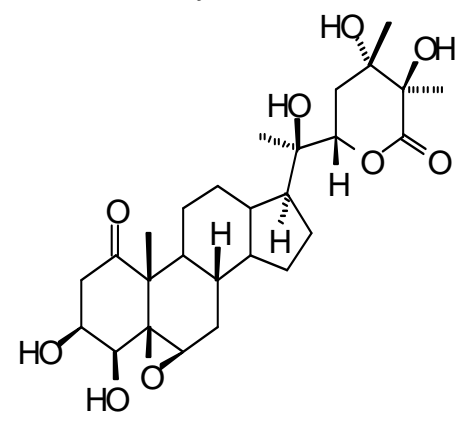

Physangulide

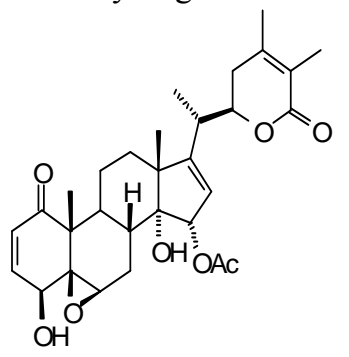

Withangulatin A

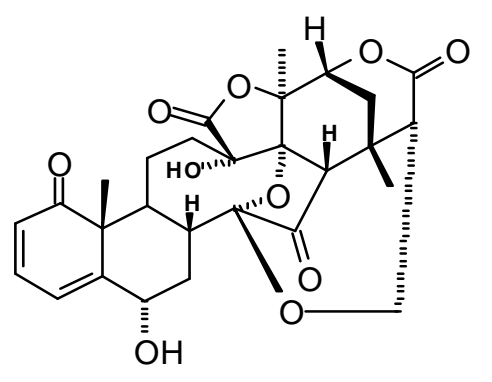

Physalin G

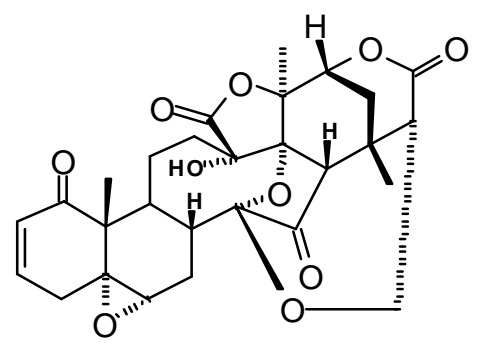

Physalin J

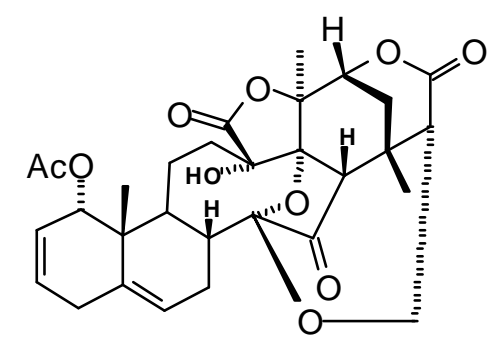

Physalin V

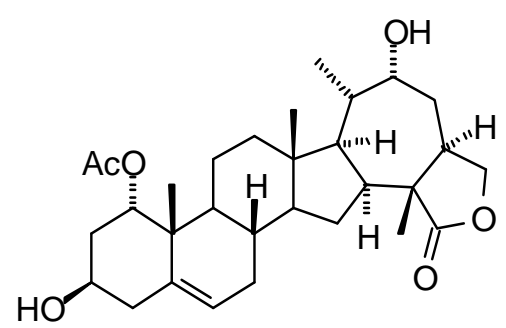

Physanolide A

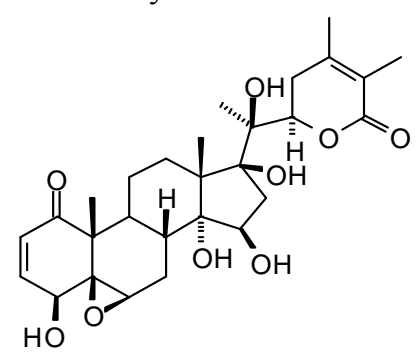

Withangulatin B

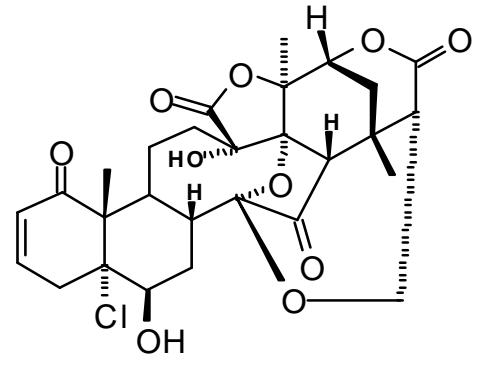

Physalin H

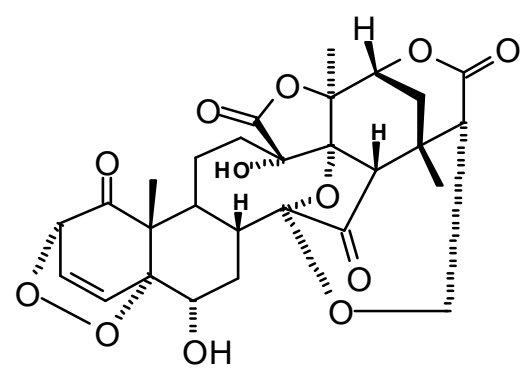

Physalin K

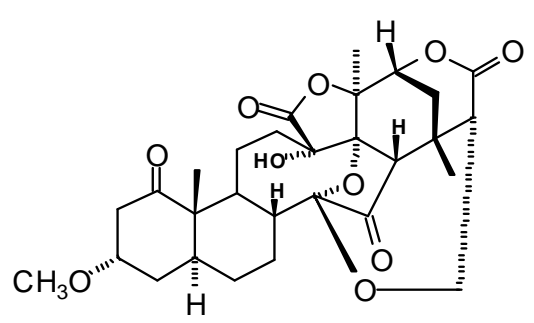

Physalin W

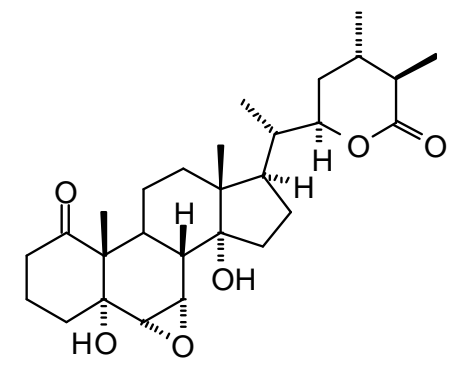

Vamonolide

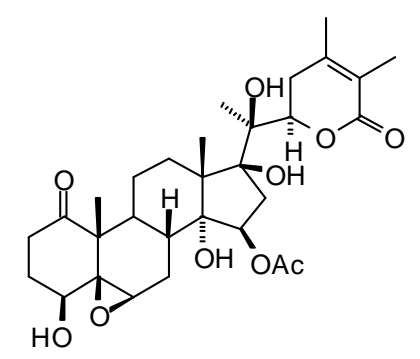

Withangulatin C 
Figure 24. Cont.

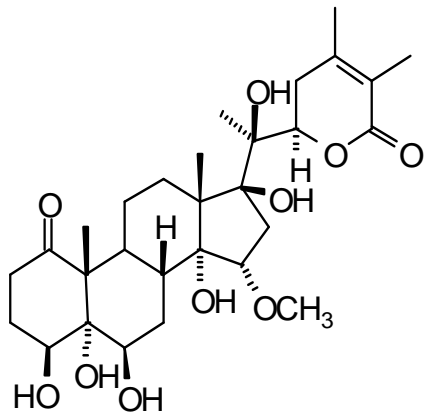

Withangulatin D

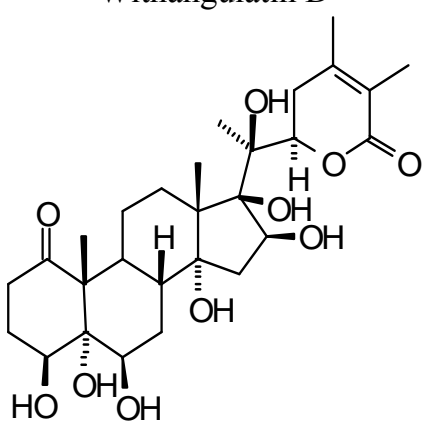

Withangulatin $G$

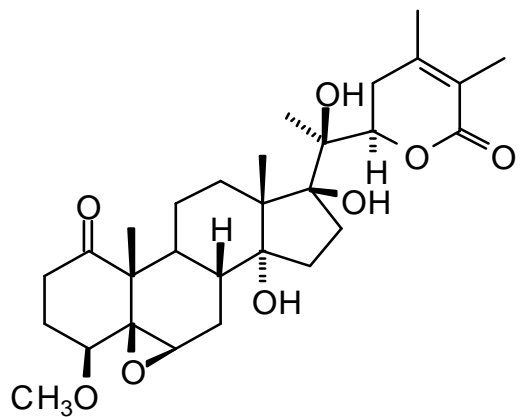

Withangulatin E

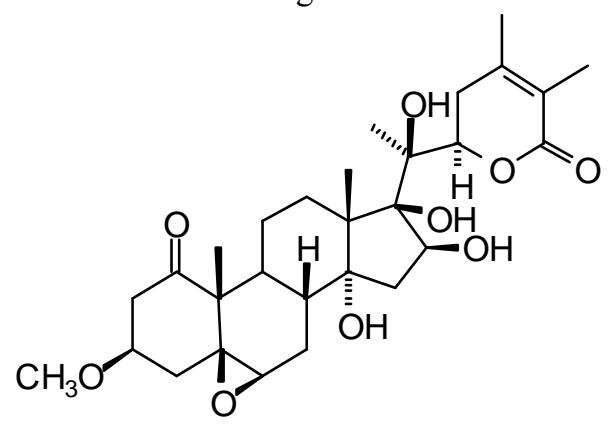

Withangulatin $\mathrm{H}$

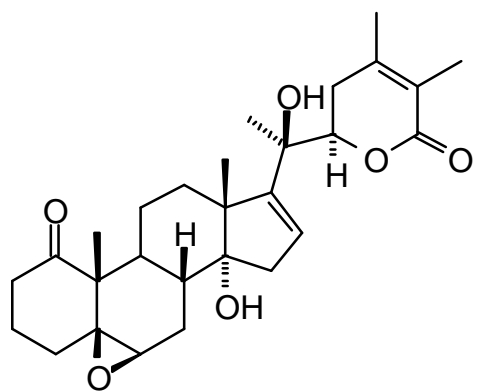

Withangulatin $\mathrm{F}$

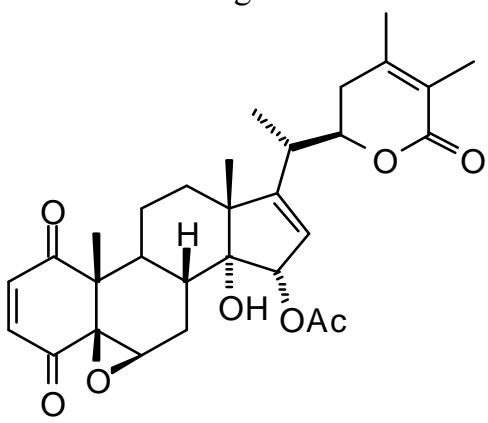

Withangulatin I

Grandifotane showed selective docking to LmajDHODH with a docking energy of $-154.4 \mathrm{~kJ} / \mathrm{mol}$. The lowest-energy pose of the ligand placed the compound at the binding site of the co-crystallized ligand, 5-nitroorotic acid (Figure 25). The furan ring of the ligand is sandwiched between the riboflavin monophosphate cofactor and Cys 131. There are hydrogen-bonding interactions between the docked ligand and residues Asn 199, Asn 68, Ser 69, and Ser 130. Grandifotane, isolated from Khaya grandifoliola [65], has apparently not been reported to be antiparasitic. The bark and seeds of K. grandifoliola, however, have shown significant antimalarial activity [66]. 6-O-Acetylswietenolide, also isolated from $K$. grandifoliola, has shown antiplasmodial activity [67], and this compound showed preferential docking to LmexGPDH. The lowest-energy docking pose of 6-O-acetylswietenolide with LmexGPDH (Figure 26) lies in a cavity surrounded by Arg 274, Phe 26, Lys 125, Phe 156, Val 298, Lys 210, and Ala 157. Lys 125 and Lys 210 have been identified as critical to catalytic activity of this enzyme [15]. With the exception of lanosterol, the steroid ligands showed preferential docking to LmajMetRS. The lowest-energy poses for these steroids show them all occupying the methionyl adenylate binding site (Figure 27). The tetracyclic steroidal structures all occupy the same position in a hydrophobic pocket surrounded by Asp 486, Trp 515, Lys 522, His 228, Gly 227, His 513, and Tyr 218, and hydrogen-bonded by way of the 3-hydroxyl group of the steroid to Trp 516 and Gly 514 . Clerosterol has shown in vitro antileishmanial activity [68], while saringosterol, stigmasterol, and 24-hydroperoxy-24-vinylcholesterol, in addition to clerosterol, have shown in vitro antitrypanosomal activity [69]. $\beta$-Sitosterol has shown modest antitrypanosomal activity [70]. 
Table 29. MolDock docking energies $(\mathrm{kJ} / \mathrm{mol})$ of limonoids with Leishmania major protein targets.

\begin{tabular}{|c|c|c|c|c|c|c|c|c|c|c|c|c|}
\hline Limonoids & LmajCatB & LmajDHODH & LmajdUTPase & LmajNDKb & LmajNH & LmajNMT & LmajOPB & LmajPDE1 & LmajPTR1 & LmajMetRS & LmajTyrRS & LmajUGPase \\
\hline $\begin{array}{l}11 \alpha-\text { Acetoxy- } 2 \alpha-\text { hydroxy }-6 \\
- \text { deoxyswietenine_acetate }\end{array}$ & -77.4 & -100.5 & -112.6 & -84.0 & -112.9 & -113.7 & -79.4 & -90.7 & -99.9 & -85.3 & -106.7 & -103.7 \\
\hline 3-O-Acetylanthothecanolide & -102.6 & -129.9 & -114.6 & -91.2 & -111.4 & -121.1 & -103.5 & -96.4 & -102.1 & -103.7 & -106.8 & -107.6 \\
\hline 3-O-Acetylkhayalactone & -70.4 & -103.4 & -96.4 & -98.9 & -115.9 & -123.4 & -96.9 & -109.5 & -109.5 & -114.5 & -106.6 & -123.7 \\
\hline 1-O-Acetylkhayanolide A & -87.7 & -103.2 & -85.4 & -86.3 & -96.5 & -114.1 & -92.2 & -93.2 & -88.7 & -81.1 & -105.0 & -106.8 \\
\hline 1-O-Acetylkhayanolide B & -80.0 & -86.6 & -87.0 & -87.1 & -80.7 & -105.0 & -105.5 & -74.5 & -72.8 & -91.8 & -95.5 & -94.5 \\
\hline 3-O-Acetylswietenine & -79.2 & -90.6 & -95.8 & -69.9 & -102.9 & -104.3 & -82.6 & -84.6 & -82.4 & -92.3 & -95.5 & -121.8 \\
\hline 3-O-Acetylswietenolide & -91.9 & -115.0 & -92.7 & -82.9 & -98.6 & -115.0 & -74.1 & -81.5 & -86.8 & -80.1 & -93.6 & -100.2 \\
\hline 6-O-Acetylswietenolide & -87.9 & -107.6 & -80.0 & -86.6 & -97.4 & -102.0 & -81.6 & -98.4 & -85.7 & -96.3 & -107.5 & -108.7 \\
\hline Anthothecanolide & -103.7 & -133.3 & -85.5 & -101.6 & -93.7 & -112.3 & -98.4 & -94.8 & -104.4 & -97.4 & -96.8 & -114.1 \\
\hline Carapa spirolactone & -92.7 & -91.4 & -87.0 & -89.7 & -99.1 & -90.6 & -93.9 & -104.0 & -71.8 & -92.1 & -95.7 & -86.5 \\
\hline Carapin & -79.9 & -109.2 & -89.9 & -95.0 & -92.8 & -95.0 & -84.8 & -92.6 & -86.4 & -88.3 & -98.9 & -110.5 \\
\hline Carapolide A & -107.8 & -140.0 & -101.1 & -114.9 & -111.7 & -119.2 & -98.5 & -113.5 & -92.4 & -125.5 & -107.1 & -123.5 \\
\hline Carapolide B & -104.9 & -128.3 & -88.1 & -117.3 & -111.7 & -117.4 & -124.7 & -106.0 & -82.3 & -98.6 & -107.9 & -107.4 \\
\hline Carapolide C & -94.5 & -105.1 & -82.7 & -106.2 & -99.2 & -110.1 & -118.5 & -109.8 & -76.3 & -95.5 & -104.4 & -107.8 \\
\hline 7-Deacetoxy-7-oxogedunin & -51.6 & -95.8 & -82.2 & -90.1 & -86.0 & -93.6 & -100.1 & -100.2 & -91.2 & -79.0 & -91.3 & -86.5 \\
\hline $\begin{array}{l}\text { 1-O-Deacetyl-6-deoxykhay } \\
\text { anolide E }\end{array}$ & -104.2 & -81.4 & -75.8 & -79.7 & -96.9 & -94.3 & -88.0 & -72.9 & -85.6 & -96.6 & -94.1 & -102.8 \\
\hline 7-Deacetylgedunin & -84.7 & -86.9 & -78.5 & -84.8 & -85.2 & -87.5 & -96.9 & -99.7 & -89.9 & -83.2 & -91.5 & -90.3 \\
\hline $\begin{array}{l}1-O \text {-Deacetyl-2 } \alpha \text {-hydroxyk } \\
\text { hayanolide } \mathrm{E}\end{array}$ & -96.4 & -105.3 & -77.1 & -74.1 & -91.7 & -101.1 & -93.7 & -81.9 & -85.9 & -97.6 & -109.8 & -108.7 \\
\hline Deacetylkhayanolide E & -104.7 & -85.6 & -76.5 & -80.6 & -88.0 & -97.2 & -87.0 & -72.6 & -83.1 & -98.8 & -110.8 & -102.9 \\
\hline 1-Deacetylkhivorin & -60.5 & -92.7 & -96.1 & -78.3 & -96.3 & -107.5 & -58.1 & -84.0 & -81.8 & -97.0 & -98.5 & -97.7 \\
\hline 3-Deacetylkhivorin & -73.2 & -95.7 & -90.4 & -71.8 & -96.0 & -110.6 & -99.1 & -99.4 & -81.4 & -82.4 & -98.7 & -108.2 \\
\hline 7-Deacetylkhivorin & -77.8 & -94.0 & -99.2 & -85.0 & -88.2 & -96.3 & -93.5 & -79.1 & -87.1 & -83.7 & -89.9 & -95.6 \\
\hline 6-Deoxyswietenolide & -84.5 & -100.2 & -79.3 & -84.0 & -97.3 & -108.8 & -90.1 & -84.6 & -93.8 & -90.9 & -98.2 & -100.5 \\
\hline 3,7-Dideacetylkhivorin & -81.5 & -93.0 & -83.4 & -64.8 & -105.3 & -96.3 & -93.9 & -109.3 & -108.1 & -76.6 & -104.4 & -99.6 \\
\hline Evodulone & -83.0 & -113.4 & -87.5 & -83.7 & -103.1 & -103.3 & -96.8 & -114.6 & -87.5 & -80.9 & -96.0 & -101.9 \\
\hline Fissinolide & -89.4 & -112.8 & -93.5 & -80.4 & -99.0 & -121.6 & -99.6 & -85.5 & -94.3 & -110.6 & -88.8 & -97.1 \\
\hline Gedunin & -74.5 & -106.8 & -81.4 & -70.2 & -89.5 & -102.1 & -93.5 & -102.8 & -96.4 & -87.1 & -93.1 & -97.7 \\
\hline
\end{tabular}


Table 29. Cont.

\begin{tabular}{|c|c|c|c|c|c|c|c|c|c|c|c|c|}
\hline Limonoids & LmajCatB & LmajDHODH & LmajdUTPase & LmajNDKb & LmajNH & LmajNMT & LmajOPB & LmajPDE1 & LmajPTR1 & LmajMetRS & LmajTyrRS & LmajUGPase \\
\hline Grandifolide A & -75.5 & -113.8 & -93.3 & -101.7 & -115.1 & -128.4 & -96.9 & -94.9 & -95.7 & -98.4 & -108.3 & -102.9 \\
\hline Grandifolin & -91.5 & -110.0 & -99.7 & -92.9 & -96.2 & -100.3 & -94.8 & -94.1 & -114.7 & -95.8 & -108.9 & -100.0 \\
\hline Grandifoliolenone & -84.6 & -88.5 & -89.0 & -88.7 & -103.9 & -106.0 & -102.8 & -97.8 & -89.6 & -86.1 & -94.2 & -119.8 \\
\hline Grandifotane & -102.5 & -154.5 & -94.6 & -80.1 & -99.5 & -106.1 & -90.4 & -107.9 & -96.4 & -96.5 & -104.0 & -112.7 \\
\hline 6-Hydroxykhayalactone & -112.4 & -105.3 & -89.5 & -111.0 & -106.7 & -116.6 & -92.8 & -88.3 & -98.9 & -101.2 & -111.5 & -126.4 \\
\hline $\begin{array}{l}3 \beta-\text { Isobutyryloxy-1-oxomel } \\
\text { iac- } 8(30) \text {-enate }\end{array}$ & -95.6 & -119.9 & -109.6 & -89.9 & -101.4 & -111.4 & -105.5 & -81.3 & -98.8 & -86.9 & -109.1 & -110.0 \\
\hline Khayalactone & -107.6 & -110.0 & -90.3 & -103.4 & -102.6 & -115.8 & -92.1 & -96.1 & -104.7 & -101.0 & -113.2 & -126.4 \\
\hline Khayanolide A & -110.8 & -123.5 & -101.3 & -104.4 & -111.1 & -115.5 & -98.4 & -105.9 & -107.9 & -138.5 & -127.4 & -123.9 \\
\hline Khayanolide B & -104.1 & -93.6 & -77.9 & -86.4 & -93.6 & -99.7 & -94.8 & -59.4 & -86.1 & -97.9 & -99.8 & -105.4 \\
\hline Khivorin & -75.6 & -98.7 & -99.2 & -91.1 & -99.1 & -101.7 & -97.5 & -77.8 & -92.1 & -89.3 & -101.3 & -109.6 \\
\hline Methyl acetoxyangolensate & -90.2 & -102.1 & -75.7 & -99.5 & -102.2 & -107.2 & -82.9 & -93.5 & -80.3 & -108.2 & -98.2 & -94.5 \\
\hline Methyl angolensate & -89.2 & -104.2 & -82.9 & -100.1 & -92.6 & -109.9 & -85.1 & -90.8 & -79.7 & -110.5 & -113.2 & -108.6 \\
\hline Methyl hydroxyangolensate & -82.9 & -91.4 & -73.0 & -102.8 & -87.6 & -109.2 & -73.0 & -84.1 & -79.5 & -100.2 & -108.8 & -101.4 \\
\hline Methyl ivorensate & -79.5 & -106.7 & -95.7 & -102.4 & -98.9 & -99.8 & -84.7 & -76.1 & -87.2 & -95.8 & -106.5 & -108.2 \\
\hline Mexicanolide & -86.9 & -103.0 & -82.7 & -79.6 & -95.4 & -111.6 & -90.8 & -90.2 & -95.6 & -100.5 & -93.9 & -103.4 \\
\hline Proceranolide & -86.5 & -110.3 & -77.8 & -96.1 & -97.0 & -108.7 & -76.0 & -91.5 & -94.3 & -91.7 & -102.2 & -102.0 \\
\hline Proceranolide butanoate & -94.0 & -119.7 & -87.9 & -93.8 & -97.5 & -119.2 & -95.9 & -103.9 & -89.2 & -90.9 & -107.1 & -106.6 \\
\hline Proceranone & -84.4 & -103.0 & -93.9 & -97.8 & -101.8 & -106.0 & -88.2 & -115.6 & -91.4 & -82.7 & -89.5 & -113.2 \\
\hline Procerin & -71.9 & -98.4 & -75.8 & -19.8 & -76.4 & -96.8 & -67.9 & -46.1 & -70.5 & -58.8 & -88.9 & -78.6 \\
\hline Seneganolide & -101.7 & -122.0 & -94.5 & -106.6 & -96.4 & -110.3 & -92.6 & -92.4 & -104.9 & -106.9 & -105.8 & -104.6 \\
\hline Swiemahogin A & -92.4 & -125.6 & -91.5 & -115.5 & -116.8 & -119.1 & -103.7 & -122.0 & -81.1 & -111.1 & -106.4 & -123.7 \\
\hline Swietenine & -93.7 & -114.2 & -99.4 & -84.6 & -113.1 & -107.1 & -88.8 & -87.7 & -87.6 & -91.1 & -106.8 & -110.9 \\
\hline Swietenolide & -85.7 & -108.1 & -80.4 & -83.7 & -97.4 & -109.0 & -71.8 & -82.8 & -84.3 & -102.3 & -97.4 & -98.9 \\
\hline $1,3,7-$ Trideacetylkhivorin & -86.0 & -95.7 & -79.2 & -76.7 & -94.8 & -88.5 & -101.1 & -97.0 & -61.9 & -81.2 & -97.1 & -84.1 \\
\hline
\end{tabular}


Table 30. MolDock docking energies (kJ/mol) of limonoids with Leishmania donovani and L. mexicana protein targets.

\begin{tabular}{|c|c|c|c|c|c|c|c|c|c|c|c|c|}
\hline Limonoids & LdonCatB & LdonCyp & LdonDHODH & LdonNMT & LmexGAPDH & LmexGPDH & LmexPGI & LmexPMM & $\begin{array}{c}\text { LmexPYK } \\
\text { Site } 1 \\
\end{array}$ & $\begin{array}{c}\text { LmexPYK } \\
\text { Site } 2 \\
\end{array}$ & $\begin{array}{c}\text { LmexPYK } \\
\text { Site } 3 \\
\end{array}$ & LmexTIM \\
\hline $\begin{array}{l}11 \alpha-\text { Acetoxy }-2 \alpha-\text { hydroxy}-6 \text {-deoxyswietenine } \\
\text { acetate }\end{array}$ & -92.2 & -85.4 & -46.3 & -82.9 & -94.0 & -99.6 & -88.0 & -121.3 & -95.5 & -103.8 & -103.9 & -81.9 \\
\hline 3-O-Acetylanthothecanolide & -100.8 & -85.9 & -54.7 & -70.9 & -108.1 & -100.9 & -94.3 & -117.9 & -110.2 & -97.0 & -94.7 & -97.5 \\
\hline 3-O-Acetylkhayalactone & -77.2 & -66.9 & -90.3 & -100.7 & -108.8 & -113.1 & -104.3 & -110.2 & -104.4 & -111.4 & -107.4 & -107.8 \\
\hline 1-O-Acetylkhayanolide A & -80.2 & -47.6 & -38.3 & -92.4 & -96.6 & -115.5 & -103.5 & -110.9 & -116.4 & -101.2 & -90.2 & -84.7 \\
\hline 1-O-Acetylkhayanolide B & -81.3 & -68.4 & -65.9 & -72.5 & -83.2 & -106.6 & -82.6 & -88.0 & -94.9 & -84.9 & -91.4 & -82.7 \\
\hline 3-O-Acetylswietenine & -75.2 & -78.2 & -57.4 & -106.0 & -74.8 & -118.6 & -81.1 & -106.8 & -101.8 & -108.3 & -103.5 & -61.4 \\
\hline 3-O-Acetylswietenolide & -88.0 & -81.2 & -37.9 & -106.8 & -72.1 & -103.8 & -83.5 & -89.0 & -98.3 & -95.5 & -96.0 & -68.2 \\
\hline 6-O-Acetylswietenolide & -86.6 & -69.6 & -58.4 & -91.1 & -82.4 & -142.7 & -92.8 & -85.9 & -110.1 & -97.9 & -95.8 & -67.7 \\
\hline Anthothecanolide & -97.5 & -66.6 & -69.0 & -92.0 & -95.0 & -99.1 & -86.8 & -118.1 & -100.7 & -98.3 & -91.0 & -91.5 \\
\hline Carapa spirolactone & -89.9 & -75.5 & -65.2 & -61.7 & -88.6 & -94.8 & -78.3 & -96.5 & -91.4 & -94.8 & -85.9 & -78.6 \\
\hline Carapin & -71.7 & -102.8 & no dock & -94.8 & -84.6 & -106.0 & -78.4 & -99.7 & -104.6 & -94.8 & -82.9 & -78.9 \\
\hline Carapolide A & -113.0 & -101.2 & -88.5 & -97.5 & -88.6 & -118.9 & -94.1 & -112.6 & -114.2 & -115.6 & -106.5 & -100.6 \\
\hline Carapolide B & -96.9 & -82.5 & -21.9 & -93.1 & -90.7 & -107.7 & -90.1 & -98.7 & -107.0 & -109.9 & -99.4 & -91.4 \\
\hline Carapolide C & -102.9 & -99.2 & -58.0 & -78.9 & -83.1 & -112.1 & -88.5 & -115.3 & -107.4 & -113.3 & -98.4 & -94.4 \\
\hline 7-Deacetoxy-7-oxogedunin & -68.4 & -66.9 & -14.8 & -79.7 & -79.6 & -92.1 & -73.6 & -83.5 & -111.5 & -101.0 & -95.5 & -83.9 \\
\hline 1-O-Deacetyl-6-deoxykhayanolide E & -101.8 & -87.2 & -70.1 & -73.5 & -82.3 & -99.9 & -82.4 & -75.6 & -97.4 & -99.5 & -91.7 & -71.8 \\
\hline 7-Deacetylgedunin & -52.7 & -81.4 & -11.3 & -82.6 & -84.8 & -91.5 & -74.1 & -82.5 & -98.3 & -93.9 & -93.3 & -80.6 \\
\hline 1-O-Deacetyl-2a-hydroxykhayanolide E & -98.1 & -58.4 & -48.5 & -64.2 & -79.0 & -111.8 & -87.0 & -84.9 & -100.4 & -104.5 & -86.2 & -66.2 \\
\hline Deacetylkhayanolide E & -96.2 & -69.1 & -56.0 & -58.8 & -84.2 & -92.6 & -85.0 & -77.8 & -97.5 & -101.8 & -88.6 & -64.2 \\
\hline 1-Deacetylkhivorin & -52.5 & -89.2 & no dock & -74.9 & -76.6 & -99.4 & -83.8 & -84.7 & -98.4 & -106.4 & -93.5 & -66.4 \\
\hline 3-Deacetylkhivorin & -68.8 & -92.2 & -66.2 & -84.2 & -96.9 & -100.5 & -99.7 & -85.4 & -98.4 & -91.3 & -79.4 & -71.6 \\
\hline 7-Deacetylkhivorin & -68.1 & -68.6 & -36.1 & -94.8 & -88.0 & -99.8 & -83.1 & -85.5 & -105.3 & -104.6 & -94.1 & -75.9 \\
\hline 6-Deoxyswietenolide & -90.3 & -75.0 & no dock & -94.3 & -74.2 & -114.6 & -84.8 & -105.7 & -90.4 & -86.9 & -94.5 & -73.0 \\
\hline 3,7-Dideacetylkhivorin & -62.6 & -69.3 & -63.2 & -87.3 & -92.3 & -96.2 & -88.0 & -78.6 & -113.5 & -85.2 & -98.5 & -73.1 \\
\hline Evodulone & -80.2 & -84.8 & -74.3 & -90.6 & -79.0 & -98.7 & -86.6 & -103.2 & -103.4 & -105.3 & -93.2 & -88.4 \\
\hline
\end{tabular}


Table 30. Cont.

\begin{tabular}{|c|c|c|c|c|c|c|c|c|c|c|c|c|}
\hline Limonoids & LdonCatB & LdonCyp & LdonDHODH & LdonNMT & LmexGAPDH & LmexGPDH & LmexPGI & LmexPMM & $\begin{array}{c}\text { LmexPYK } \\
\text { Site } 1\end{array}$ & $\begin{array}{c}\text { LmexPYK } \\
\text { Site } 2\end{array}$ & $\begin{array}{c}\text { LmexPYK } \\
\text { Site } 3\end{array}$ & LmexTIM \\
\hline Fissinolide & -83.6 & -80.3 & -54.6 & -102.4 & -64.2 & -95.1 & -85.1 & -111.9 & -90.4 & -95.2 & -92.9 & -78.1 \\
\hline Gedunin & -84.0 & -88.2 & no dock & -88.3 & -85.0 & -98.3 & -80.4 & -82.0 & -88.4 & -99.1 & -95.6 & -88.9 \\
\hline Grandifolide A & -77.1 & -96.3 & no dock & -98.1 & -91.2 & -103.8 & -90.9 & -93.4 & -104.5 & -115.5 & -87.3 & -85.8 \\
\hline Grandifolin & -53.7 & -94.9 & -67.0 & -73.2 & -83.7 & -109.1 & -89.4 & -95.4 & -105.1 & -98.9 & -104.0 & -99.3 \\
\hline Grandifoliolenone & -93.7 & -93.8 & -68.9 & -77.3 & -78.7 & -115.1 & -80.0 & -101.4 & -115.4 & -103.6 & -100.1 & -87.3 \\
\hline Grandifotane & -99.7 & -70.8 & -40.6 & -99.4 & -107.8 & -113.7 & -87.5 & -117.2 & -100.5 & -100.6 & -106.8 & -29.1 \\
\hline 6-Hydroxykhayalactone & -106.7 & -80.2 & -88.2 & -96.4 & -94.0 & -106.1 & -97.3 & -98.6 & -103.4 & -110.1 & -91.2 & -95.9 \\
\hline $3 \beta$-Isobutyryloxy-1-oxomeliac- $8(30)$-enate & -92.4 & -82.3 & no dock & -99.8 & -87.6 & -99.2 & -87.8 & -89.7 & -95.3 & -117.6 & -92.1 & -60.0 \\
\hline Khayalactone & -107.0 & -68.1 & -55.1 & -97.7 & -94.6 & -116.0 & -95.9 & -102.0 & -100.2 & -100.8 & -95.6 & -94.2 \\
\hline Khayanolide A & -110.9 & -98.7 & -18.3 & -97.8 & -92.5 & -137.8 & -98.6 & -112.1 & -115.3 & -107.8 & -107.3 & -89.9 \\
\hline Khayanolide B & -102.5 & -72.5 & -39.5 & -70.4 & -90.8 & -94.6 & -88.3 & -77.6 & -88.2 & -105.2 & -94.7 & -71.2 \\
\hline Khivorin & -64.6 & -89.4 & -72.0 & -30.2 & -92.4 & -107.4 & -82.0 & -84.0 & -93.9 & -108.2 & -85.6 & -78.8 \\
\hline Methyl acetoxyangolensate & -89.1 & -58.8 & -59.5 & -88.8 & -98.0 & -94.6 & -72.7 & -81.8 & -108.9 & -98.4 & -94.8 & -41.3 \\
\hline Methyl angolensate & -89.5 & -52.0 & -74.5 & -27.42 & -98.6 & -112.9 & -75.3 & -109.7 & -99.1 & -95.9 & -87.2 & -79.6 \\
\hline Methyl hydroxyangolensate & -91.0 & -73.9 & -74.7 & -75.6 & -94.5 & -115.7 & -76.6 & -112.5 & -93.1 & -93.7 & -87.6 & -78.9 \\
\hline Methyl ivorensate & -86.9 & -75.4 & -63.9 & -82.2 & -102.0 & -119.7 & -77.1 & -80.8 & -104.0 & -91.3 & -96.2 & -70.8 \\
\hline Mexicanolide & -85.4 & -67.6 & -33.9 & -84.3 & -67.9 & -112.8 & -92.2 & -105.5 & -97.3 & -90.4 & -98.3 & -59.4 \\
\hline Proceranolide & -90.2 & -77.6 & no dock & -94.7 & -66.8 & -121.6 & -87.2 & -106.0 & -90.0 & -88.8 & -94.6 & -73.9 \\
\hline Proceranolide butanoate & -94.7 & -81.0 & -70.3 & -111.8 & -76.2 & -92.9 & -89.1 & -95.1 & -100.9 & -106.8 & -107.4 & -63.0 \\
\hline Proceranone & -87.7 & -89.0 & no dock & -84.6 & -75.0 & -99.3 & -86.0 & -109.9 & -98.0 & -108.4 & -87.7 & -98.6 \\
\hline Procerin & -77.5 & -22.4 & -59.7 & -107.5 & -94.8 & -111.3 & -108.8 & -84.5 & -93.9 & -86.6 & -70.7 & -64.8 \\
\hline Seneganolide & -99.5 & -92.0 & -51.9 & -88.4 & -89.9 & -98.8 & -86.6 & -109.8 & -95.9 & -92.5 & -92.5 & -83.5 \\
\hline Swiemahogin A & -69.7 & -94.9 & -52.2 & -81.4 & -79.9 & -125.4 & -92.8 & -117.4 & -120.1 & -106.2 & -110.0 & -91.4 \\
\hline Swietenine & -90.7 & -67.4 & no dock & -99.3 & -85.0 & -104.1 & -86.3 & -91.6 & -99.6 & -122.3 & -100.7 & -78.0 \\
\hline Swietenolide & -87.3 & -72.3 & no dock & -93.6 & -72.3 & -121.0 & -88.9 & -94.4 & -99.2 & -91.2 & -87.7 & -68.3 \\
\hline 1,3,7-Trideacetylkhivorin & -53.7 & -80.5 & no dock & -76.2 & -87.1 & -97.2 & -81.2 & -71.5 & -96.9 & -93.0 & -82.8 & -83.2 \\
\hline
\end{tabular}


Table 31. MolDock docking energies (kJ/mol) of limonoids with Leishmania infantum protein targets.

\begin{tabular}{|c|c|c|c|c|c|}
\hline Limonoids & LinfCYP51 & LinfGLO2 & LinfPnC1 & LinfTDR1 & LinfTR \\
\hline $\begin{array}{l}11 \alpha-\text { Acetoxy }-2 \alpha-\text { hydroxy- }-6 \text {-deoxyswietenine } \\
\text { acetate }\end{array}$ & -98.0 & -75.1 & no dock & -93.6 & -89.4 \\
\hline 3-O-Acetylanthothecanolide & -102.5 & -77.7 & no dock & -82.5 & -101.6 \\
\hline 3-O-Acetylkhayalactone & -116.2 & -99.5 & no dock & -103.7 & -113.2 \\
\hline 1-O-Acetylkhayanolide A & -102.4 & -89.1 & no dock & -87.9 & -108.3 \\
\hline 1-O-Acetylkhayanolide B & -114.3 & -82.0 & no dock & -80.6 & -84.0 \\
\hline 3-O-Acetylswietenine & -95.3 & -74.9 & no dock & -76.4 & -84.2 \\
\hline 3-O-Acetylswietenolide & -105.4 & -80.3 & no dock & -79.5 & -95.4 \\
\hline 6-O-Acetylswietenolide & -103.1 & -77.4 & no dock & -91.1 & -92.6 \\
\hline Anthothecanolide & -107.9 & -74.6 & -57.2 & -82.9 & -94.3 \\
\hline Carapa spirolactone & -108.8 & -69.7 & no dock & -68.3 & -87.3 \\
\hline Carapin & -94.3 & -80.2 & no dock & -80.9 & -86.3 \\
\hline Carapolide A & -111.8 & -89.1 & no dock & -98.1 & -98.3 \\
\hline Carapolide B & -103.1 & -71.6 & no dock & -91.8 & -92.9 \\
\hline Carapolide C & -106.9 & -80.4 & no dock & -79.4 & -92.5 \\
\hline 7-Deacetoxy-7-oxogedunin & -104.3 & -71.9 & no dock & -70.0 & -84.6 \\
\hline 1-O-Deacetyl-6-deoxykhayanolide $\mathrm{E}$ & -98.0 & -77.3 & no dock & -78.6 & -90.9 \\
\hline 7-Deacetylgedunin & -91.2 & -72.8 & no dock & -72.6 & -80.4 \\
\hline $1-O$-Deacetyl $-2 \alpha$-hydroxykhayanolide $\mathrm{E}$ & -110.6 & -75.1 & no dock & -83.8 & -88.6 \\
\hline Deacetylkhayanolide E & -103.4 & -81.0 & -45.4 & -83.0 & -84.9 \\
\hline 1-Deacetylkhivorin & -101.2 & -72.0 & no dock & -75.8 & -87.7 \\
\hline 3-Deacetylkhivorin & -106.0 & -76.8 & no dock & -76.0 & -98.3 \\
\hline 7-Deacetylkhivorin & -94.5 & -83.8 & no dock & -87.0 & -73.7 \\
\hline 6-Deoxyswietenolide & -95.9 & -76.5 & no dock & -83.0 & -92.4 \\
\hline 3,7-Dideacetylkhivorin & -95.9 & -74.8 & no dock & -76.3 & -103.3 \\
\hline Evodulone & -105.3 & -81.6 & no dock & -88.6 & -87.5 \\
\hline Fissinolide & -99.2 & -78.0 & no dock & -78.8 & -99.0 \\
\hline Gedunin & -107.4 & -75.9 & no dock & -82.2 & -78.4 \\
\hline
\end{tabular}


Table 31. Cont.

\begin{tabular}{|c|c|c|c|c|c|}
\hline Limonoids & LinfCYP51 & LinfGLO2 & LinfPnC1 & LinfTDR1 & LinfTR \\
\hline Grandifolide A & -109.3 & -89.6 & no dock & -90.2 & -99.8 \\
\hline Grandifolin & -105.8 & -76.1 & no dock & -75.8 & -96.8 \\
\hline Grandifoliolenone & -98.7 & -90.7 & -43.1 & -75.9 & -92.7 \\
\hline Grandifotane & -111.5 & -80.8 & no dock & -89.8 & -90.0 \\
\hline 6-Hydroxykhayalactone & -116.0 & -83.1 & no dock & -100.2 & -98.6 \\
\hline $3 \beta$-Isobutyryloxy-1-oxomeliac-8(30)-enate & -102.7 & -78.4 & no dock & -82.5 & -103.4 \\
\hline Khayalactone & -101.0 & -90.6 & no dock & -100.3 & -97.5 \\
\hline Khayanolide A & -104.5 & -83.4 & no dock & -84.3 & -97.4 \\
\hline Khayanolide B & -110.0 & -77.4 & -44.1 & -97.2 & -103.1 \\
\hline Khivorin & -100.9 & -71.4 & no dock & -82.0 & -93.8 \\
\hline Methyl acetoxyangolensate & -99.6 & -66.4 & no dock & -84.9 & -82.8 \\
\hline Methyl angolensate & -90.5 & -71.0 & no dock & -77.8 & -85.6 \\
\hline Methyl hydroxyangolensate & -95.8 & -65.2 & -48.5 & -78.2 & -85.4 \\
\hline Methyl ivorensate & -95.5 & -77.8 & no dock & -85.2 & -92.5 \\
\hline Mexicanolide & -90.4 & -78.8 & -27.5 & -75.8 & -92.3 \\
\hline Proceranolide & -95.7 & -78.9 & no dock & -83.1 & -91.5 \\
\hline Proceranolide butanoate & -100.6 & -82.1 & no dock & -89.5 & -93.5 \\
\hline Proceranone & -107.9 & -89.7 & no dock & -90.7 & -94.6 \\
\hline Procerin & -94.9 & -70.1 & no dock & -68.8 & -81.8 \\
\hline Seneganolide & -101.8 & -76.9 & no dock & -82.0 & -86.4 \\
\hline Swiemahogin A & -113.3 & -84.7 & no dock & -93.4 & -97.4 \\
\hline Swietenine & -108.3 & -79.6 & no dock & -83.2 & -87.1 \\
\hline Swietenolide & -97.0 & -72.7 & no dock & -86.3 & -93.3 \\
\hline 1,3,7-Trideacetylkhivorin & -92.0 & -73.6 & no dock & -62.4 & -86.6 \\
\hline
\end{tabular}


Table 32. MolDock docking energies $(\mathrm{kJ} / \mathrm{mol})$ of withanolides with Leishmania major protein targets.

\begin{tabular}{|c|c|c|c|c|c|c|c|c|c|c|c|c|}
\hline Withanolides & LmajCatB & LmajDHODH & LmajdUTPase & LmajNDKb & LmajNH & LmajNMT & LmajOPB & LmajPDE1 & LmajPTR1 & LmajMetRS & LmajTyrRS & LmajUGPase \\
\hline 24,25-Epoxywithanolide D & -92.9 & -121.0 & -109.3 & -104.7 & -104.2 & -124.1 & -102.7 & -114.1 & -114.2 & -98.2 & -119.5 & -108.2 \\
\hline 14-Hydroxyixocarpanolide & -77.9 & -104.9 & -89.2 & -77.9 & -100.9 & -98.2 & -90.0 & -113.0 & -81.8 & -99.0 & -99.6 & -106.6 \\
\hline Physagulin A & -102.7 & -123.7 & -98.3 & -103.1 & -108.2 & -113.7 & -100.6 & -115.8 & -110.0 & -98.4 & -104.0 & -110.6 \\
\hline Physagulin B & -97.0 & -107.6 & -89.8 & -105.1 & -104.5 & -119.6 & -95.6 & -103.5 & -93.8 & -114.8 & -106.0 & -111.0 \\
\hline Physagulin C & -93.4 & -99.5 & -89.6 & -105.5 & -106.5 & -113.8 & -95.9 & -118.3 & -93.8 & -127.3 & -105.0 & -122.7 \\
\hline Physagulin F & -92.8 & -88.2 & -88.0 & -96.5 & -106.1 & -102.6 & -96.9 & -107.5 & -90.9 & -133.2 & -98.8 & -98.5 \\
\hline Physagulin $\mathrm{H}$ & -91.8 & -112.0 & -88.3 & -92.9 & -105.4 & -111.2 & -103.2 & -111.8 & -95.5 & -126.1 & -104.1 & -112.8 \\
\hline Physagulin I & -94.2 & -107.1 & -92.3 & -99.1 & -107.6 & -103.0 & -97.3 & -110.6 & -92.1 & -123.8 & -103.8 & -106.2 \\
\hline Physagulin J & -96.8 & -105.4 & -87.2 & -90.6 & -96.5 & -106.7 & -94.5 & -117.6 & -91.2 & -105.6 & -100.1 & -110.1 \\
\hline Physagulin K & -97.5 & -118.5 & -81.7 & -69.4 & -111.2 & -112.6 & -108.9 & -108.9 & -95.5 & -101.7 & -97.3 & -104.1 \\
\hline Physagulin L & -98.8 & -114.8 & -88.0 & -69.9 & -111.2 & -112.1 & -110.3 & -97.3 & -106.0 & -103.0 & -99.2 & -107.6 \\
\hline Physagulin L' & -107.0 & -109.0 & -104.2 & -99.2 & -99.7 & -114.2 & -108.2 & -111.9 & -108.2 & -115.0 & -103.5 & -101.3 \\
\hline Physagulin M & -72.5 & -95.0 & -90.9 & -83.2 & -104.8 & -110.0 & -88.3 & -95.8 & -89.1 & -92.9 & -103.3 & -115.4 \\
\hline Physagulin $\mathrm{M}^{\prime}$ & -88.5 & -115.0 & -93.6 & -99.3 & -98.2 & -112.8 & -92.4 & -106.9 & -97.1 & -120.2 & -100.5 & -101.0 \\
\hline Physagulin N & -93.1 & -102.8 & -92.3 & -93.1 & -101.9 & -115.2 & -94.7 & -108.5 & -84.4 & -110.6 & -106.6 & -118.1 \\
\hline Physagulin $\mathrm{N}^{\prime}$ & -94.4 & -102.5 & -72.7 & -94.6 & -99.7 & -99.0 & -96.1 & -116.1 & -90.0 & -110.4 & -90.2 & -91.9 \\
\hline Physagulin $\mathrm{O}$ & -87.3 & -94.3 & -81.5 & -91.4 & -100.8 & -103.6 & -95.0 & -98.0 & -87.0 & -88.9 & -95.5 & -108.1 \\
\hline Physalin A & -86.5 & -92.6 & -71.2 & -86.8 & -87.3 & -92.0 & -110.7 & -70.2 & -75.0 & -84.2 & -90.0 & -101.2 \\
\hline Physalin B & -76.9 & -92.3 & -80.8 & -62.9 & -90.0 & -100.2 & -86.9 & -95.8 & -76.7 & -78.1 & -88.3 & -101.7 \\
\hline Physalin D & -83.9 & -87.6 & -85.1 & -65.4 & -101.7 & -99.7 & -86.9 & -85.4 & -68.4 & -83.2 & -83.8 & -96.5 \\
\hline Physalin E & -82.1 & -91.5 & -85.3 & -65.5 & -88.5 & -100.6 & -94.1 & -76.3 & -66.7 & -83.2 & -82.2 & -99.1 \\
\hline Physalin F & -81.2 & -90.1 & -80.7 & -66.0 & -93.0 & -101.4 & -83.5 & -96.3 & -73.2 & -78.0 & -96.7 & -101.7 \\
\hline Physalin G & -95.6 & -103.9 & -80.1 & -82.3 & -96.3 & -89.6 & -91.7 & -91.6 & -88.0 & -89.8 & -103.5 & -103.8 \\
\hline Physalin H & -82.8 & -89.5 & -80.4 & -65.0 & -98.4 & -95.8 & -86.2 & -86.6 & -67.2 & -80.8 & -87.1 & -93.0 \\
\hline Physalin I & -78.8 & -85.4 & -82.6 & -71.4 & -85.9 & -93.0 & -87.3 & -75.2 & -60.5 & -79.5 & -82.5 & -98.4 \\
\hline Physalin J & -80.2 & -91.0 & -85.1 & -74.9 & -96.4 & -103.1 & -86.6 & -91.8 & -73.9 & -77.7 & -84.1 & -94.1 \\
\hline Physalin K & -83.1 & -106.9 & -73.7 & -81.7 & -87.5 & -88.7 & -66.7 & -68.5 & -71.5 & -77.6 & -81.3 & -105.3 \\
\hline Physalin U & -81.2 & -96.3 & -83.0 & -50.7 & -93.4 & -93.0 & -90.4 & -99.2 & -74.5 & -85.2 & -91.9 & -103.1 \\
\hline
\end{tabular}


Table 32. Cont.

\begin{tabular}{|c|c|c|c|c|c|c|c|c|c|c|c|c|}
\hline Withanolides & LmajCatB & LmajDHODH & LmajdUTPase & LmajNDKb & LmajNH & LmajNMT & LmajOPB & LmajPDE1 & LmajPTR1 & LmajMetRS & LmajTyrRS & LmajUGPase \\
\hline Physalin V & -88.4 & -101.1 & -83.0 & -83.5 & -103.5 & -100.9 & -96.4 & -95.5 & -79.4 & -84.8 & -86.9 & -107.5 \\
\hline Physalin W & -93.4 & -95.0 & -85.1 & -75.9 & -91.0 & -92.0 & -78.6 & -100.4 & -80.2 & -84.0 & -91.7 & -110.3 \\
\hline Physangulide & -101.9 & -88.9 & -104.0 & -97.8 & -101.8 & -109.1 & -92.9 & -117.4 & -110.6 & -104.4 & -96.4 & -101.8 \\
\hline Physanolide A & -85.7 & -102.9 & -84.6 & -69.0 & -97.6 & -107.9 & -93.8 & -103.5 & -100.5 & -94.5 & -88.4 & -101.1 \\
\hline Vamonolide & -83.4 & -96.7 & -87.8 & -109.9 & -95.7 & -103.8 & -96.5 & -109.0 & -84.4 & -92.2 & -106.7 & -97.4 \\
\hline Withangulatin A & -92.4 & -121.9 & -97.8 & -108.1 & -110.6 & -124.2 & -97.1 & -112.9 & -110.1 & -99.4 & -105.1 & -112.2 \\
\hline Withangulatin B & -105.4 & -89.8 & -96.1 & -96.6 & -95.7 & -99.9 & -94.3 & -107.2 & -96.2 & -117.9 & -104.7 & -91.0 \\
\hline Withangulatin C & -85.7 & -93.6 & -86.6 & -100.7 & -90.1 & -109.7 & -106.3 & -89.2 & -94.1 & -125.9 & -104.6 & -98.5 \\
\hline Withangulatin D & -88.8 & -80.3 & -89.4 & -102.6 & -96.6 & -107.2 & -92.0 & -113.3 & -86.9 & -126.2 & -105.2 & -104.8 \\
\hline Withangulatin E & -57.3 & -94.8 & -36.3 & -92.5 & -85.7 & -102.4 & -89.1 & -95.7 & -88.9 & -96.0 & -112.7 & -97.9 \\
\hline Withangulatin F & -85.1 & -96.0 & -83.8 & -103.9 & -97.7 & -98.9 & -87.9 & -120.8 & -85.8 & -96.0 & -102.4 & -96.9 \\
\hline Withangulatin G & -91.5 & -122.3 & -76.0 & -100.4 & -95.2 & -100.1 & -93.7 & -99.7 & -87.6 & -97.7 & -105.8 & -92.5 \\
\hline Withangulatin $\mathrm{H}$ & -91.6 & -105.5 & -89.1 & -96.8 & -81.0 & -94.8 & -105.1 & -88.8 & -71.0 & -115.3 & -102.2 & -106.9 \\
\hline Withangulatin I & -98.8 & -112.4 & -90.0 & -91.3 & -106.6 & -113.1 & -88.1 & -105.6 & -106.0 & -98.2 & -102.3 & -110.7 \\
\hline
\end{tabular}

Table 33. MolDock docking energies $(\mathrm{kJ} / \mathrm{mol})$ of withanolides with Leishmania donovani and L. mexicana protein targets.

\begin{tabular}{|c|c|c|c|c|c|c|c|c|c|c|c|c|}
\hline Withanolides & LdonCatB & LdonCyp & LdonDHODH & LdonNMT & LmexGAPDH & LmexGPDH & LmexPGI & LmexPMM & $\begin{array}{c}\text { LmexPYK } \\
\text { Site } 1 \\
\end{array}$ & $\begin{array}{c}\text { LmexPYK } \\
\text { Site } 2 \\
\end{array}$ & $\begin{array}{c}\text { LmexPYK } \\
\text { Site } 3\end{array}$ & LmexTIM \\
\hline 24,25-Epoxywithanolide D & -95.7 & -101.5 & -63.4 & -103.0 & -97.7 & -118.1 & -110.4 & -125.7 & -119.7 & -106.2 & -113.6 & -95.4 \\
\hline 14-Hydroxyixocarpanolide & -88.0 & -92.8 & -30.7 & -52.2 & -80.7 & -102.3 & -77.5 & -95.6 & -103.6 & -92.2 & -100.3 & -74.3 \\
\hline Physagulin A & -97.4 & -103.3 & -82.8 & -91.9 & -91.4 & -116.2 & -100.5 & -119.2 & -111.6 & -104.4 & -106.5 & -85.0 \\
\hline Physagulin B & -105.4 & -104.2 & -85.9 & -89.9 & -94.2 & -107.6 & -96.3 & -114.4 & -110.3 & -109.3 & -103.8 & -82.9 \\
\hline Physagulin C & -98.7 & -86.0 & -80.6 & -104.7 & -91.6 & -108.7 & -98.2 & -124.7 & -103.4 & -100.7 & -103.8 & -85.9 \\
\hline Physagulin F & -94.8 & -88.7 & -84.6 & -93.6 & -88.7 & -106.0 & -87.9 & -107.8 & -105.8 & -106.3 & -91.6 & -82.3 \\
\hline Physagulin H & -96.5 & -85.2 & -83.6 & -98.8 & -86.0 & -101.4 & -97.2 & -113.0 & -106.2 & -104.6 & -101.7 & -79.3 \\
\hline Physagulin I & -95.9 & -87.5 & -77.2 & -94.7 & -90.2 & -99.9 & -91.5 & -102.3 & -101.2 & -103.5 & -95.0 & -77.7 \\
\hline Physagulin J & -94.4 & -84.6 & -60.7 & -92.5 & -88.5 & -108.7 & -91.2 & -100.6 & -98.5 & -111.2 & -100.6 & -86.6 \\
\hline Physagulin K & -85.1 & -95.8 & no dock & -92.5 & -94.2 & -109.1 & -90.1 & -92.6 & -112.2 & -106.4 & -97.1 & -76.0 \\
\hline Physagulin L & -90.6 & -83.3 & -67.6 & -97.6 & -96.2 & -112.3 & -98.2 & -104.5 & -117.3 & -103.6 & -92.0 & -68.1 \\
\hline
\end{tabular}


Table 33. Cont.

\begin{tabular}{|c|c|c|c|c|c|c|c|c|c|c|c|c|}
\hline Withanolides & LdonCatB & LdonCyp & LdonDHODH & LdonNMT & LmexGAPDH & LmexGPDH & LmexPGI & LmexPMM & $\begin{array}{c}\text { LmexPYK } \\
\text { Site } 1 \\
\end{array}$ & $\begin{array}{c}\text { LmexPYK } \\
\text { Site } 2 \\
\end{array}$ & $\begin{array}{c}\text { LmexPYK } \\
\text { Site } 3 \\
\end{array}$ & LmexTIM \\
\hline Physagulin L' & -108.8 & -104.3 & -102.2 & -94.7 & -94.2 & -110.7 & -87.0 & -109.4 & -111.5 & -98.2 & -105.4 & -101.2 \\
\hline Physagulin M & -91.2 & -91.3 & -70.6 & -81.4 & -96.1 & -101.8 & -78.6 & -87.4 & -105.6 & -111.5 & -101.9 & -75.9 \\
\hline Physagulin $\mathrm{M}^{\prime}$ & -84.3 & -94.8 & -30.7 & -90.9 & -89.8 & -106.2 & -88.9 & -88.5 & -110.5 & -103.7 & -96.3 & -106.7 \\
\hline Physagulin N & -94.9 & -99.8 & -89.5 & -95.8 & -95.0 & -106.2 & -80.7 & -115.1 & -105.1 & -112.2 & -95.4 & -82.5 \\
\hline Physagulin $\mathrm{N}^{\prime}$ & -93.2 & -83.6 & -65.4 & -85.2 & -86.2 & -112.3 & -83.4 & -103.2 & -107.5 & -103.8 & -85.1 & -55.3 \\
\hline Physagulin $\mathrm{O}$ & -90.3 & -88.8 & -88.3 & -93.9 & -93.4 & -103.5 & -84.5 & -94.6 & -99.4 & -102.4 & -106.3 & -72.0 \\
\hline Physalin A & -85.6 & -71.4 & -41.9 & -82.3 & -83.6 & -93.8 & -82.0 & -102.9 & -88.1 & -100.4 & -91.4 & -81.7 \\
\hline Physalin B & -84.9 & -71.7 & -48.5 & -81.8 & -78.4 & -88.5 & -84.8 & -92.6 & -92.0 & -89.4 & -84.8 & -73.9 \\
\hline Physalin D & -82.6 & -79.1 & -54.4 & -80.5 & -79.6 & -91.8 & -84.5 & -85.0 & -89.8 & -95.0 & -79.9 & -71.7 \\
\hline Physalin E & -83.4 & -63.3 & -30.0 & -75.9 & -81.3 & -83.5 & -74.5 & -83.7 & -96.2 & -93.5 & -81.5 & -76.0 \\
\hline Physalin F & -83.7 & -73.8 & -57.4 & -83.2 & -80.7 & -92.8 & -92.4 & -85.1 & -90.9 & -91.0 & -76.6 & -74.9 \\
\hline Physalin G & -93.6 & -71.0 & -81.2 & -92.5 & -76.9 & -78.4 & -87.9 & -97.9 & -100.6 & -90.4 & -93.0 & -69.2 \\
\hline Physalin H & -85.2 & -60.7 & no dock & -73.0 & -81.0 & -92.5 & -86.0 & -81.7 & -92.6 & -89.6 & -81.2 & -70.1 \\
\hline Physalin I & -84.2 & -51.6 & no dock & -68.3 & -82.5 & -96.2 & -87.4 & -79.0 & -84.0 & -86.5 & -82.9 & -61.6 \\
\hline Physalin J & -83.1 & -80.1 & -55.5 & -79.5 & -82.2 & -92.4 & -78.2 & -97.8 & -97.0 & -94.9 & -88.0 & -72.1 \\
\hline Physalin K & -82.6 & -74.7 & -65.5 & -84.2 & -70.4 & -90.2 & -77.6 & -76.5 & -96.7 & -87.4 & -94.2 & -60.7 \\
\hline Physalin U & -83.0 & -65.1 & -68.5 & -94.0 & -85.9 & -97.6 & -93.2 & -85.0 & -97.0 & -93.2 & -85.9 & -81.6 \\
\hline Physalin V & -83.2 & -83.6 & -27.1 & -84.3 & -81.2 & -93.9 & -93.1 & -82.8 & -95.0 & -92.7 & -90.5 & -52.8 \\
\hline Physalin W & -93.9 & -67.5 & -77.2 & -89.7 & -84.0 & -101.5 & -92.1 & -91.4 & -98.1 & -99.0 & -86.9 & -80.2 \\
\hline Physangulide & -103.2 & -99.4 & -71.3 & -106.0 & -89.9 & -105.2 & -83.7 & -111.5 & -114.6 & -108.0 & -103.3 & -82.8 \\
\hline Physanolide A & -93.4 & -96.3 & -22.9 & -103.0 & -84.0 & -99.6 & -81.4 & -106.3 & -97.4 & -96.3 & -86.2 & -44.5 \\
\hline Vamonolide & -83.4 & -89.7 & -52.6 & -90.2 & -75.4 & -96.2 & -81.5 & -107.1 & -101.6 & -100.9 & -90.8 & -77.4 \\
\hline Withangulatin A & -106.1 & -102.6 & -39.9 & -98.6 & -96.7 & -119.8 & -105.4 & -122.0 & -109.2 & -102.9 & -107.5 & -87.8 \\
\hline Withangulatin B & -95.7 & -90.0 & no dock & -94.6 & -87.5 & -99.4 & -85.5 & -94.2 & -99.6 & -106.1 & -112.4 & -92.4 \\
\hline Withangulatin $\mathrm{C}$ & -91.7 & -87.1 & -27.3 & -90.6 & -88.3 & -110.5 & -87.0 & -99.0 & -106.2 & -101.2 & -113.8 & -78.8 \\
\hline Withangulatin D & -101.2 & -92.4 & no dock & -93.6 & -84.6 & -105.1 & -86.5 & -96.2 & -92.3 & -104.8 & -108.2 & -86.5 \\
\hline Withangulatin E & -81.0 & -77.9 & -25.9 & -95.5 & -76.9 & -105.7 & -85.8 & -99.9 & -102.7 & -101.4 & -100.8 & -87.8 \\
\hline Withangulatin F & -54.3 & -94.3 & -58.6 & -83.3 & -88.7 & -108.4 & -88.3 & -100.2 & -106.2 & -97.7 & -104.7 & -99.5 \\
\hline Withangulatin G & -91.5 & -87.6 & no dock & -96.1 & -74.2 & -100.9 & -85.5 & -100.5 & -99.8 & -103.2 & -95.7 & -64.3 \\
\hline Withangulatin $\mathrm{H}$ & -95.2 & -85.5 & -65.1 & -85.9 & -80.3 & -102.5 & -75.5 & -98.8 & -95.8 & -106.9 & -97.6 & -80.7 \\
\hline Withangulatin I & -93.5 & -95.3 & -82.6 & -93.6 & -95.5 & -113.7 & -89.3 & -114.2 & -116.1 & -105.4 & -94.5 & -71.1 \\
\hline
\end{tabular}


Table 34. MolDock docking energies $(\mathrm{kJ} / \mathrm{mol})$ of withanolides with Leishmania infantum protein targets.

\begin{tabular}{|c|c|c|c|c|c|}
\hline Withanolides & LinfCYP51 & LinfGLO2 & LinfPnC1 & LinfTDR1 & LinfTR \\
\hline 24,25-Epoxywithanolide D & -128.1 & -102.8 & no dock & -103.8 & -98.4 \\
\hline 14-Hydroxyixocarpanolide & -111.7 & -81.1 & no dock & -85.3 & -94.4 \\
\hline Physagulin A & -106.7 & -88.5 & no dock & -103.6 & -102.7 \\
\hline Physagulin B & -107.7 & -82.8 & no dock & -99.0 & -95.1 \\
\hline Physagulin C & -122.5 & -98.8 & no dock & -99.7 & -94.2 \\
\hline Physagulin F & -108.2 & -70.6 & no dock & -80.8 & -96.1 \\
\hline Physagulin $\mathrm{H}$ & -114.9 & -92.6 & no dock & -96.8 & -91.9 \\
\hline Physagulin I & -102.9 & -75.1 & no dock & -84.5 & -97.7 \\
\hline Physagulin J & -115.4 & -79.4 & no dock & -87.5 & -93.2 \\
\hline Physagulin K & -105.4 & -76.4 & no dock & -88.5 & -95.0 \\
\hline Physagulin L & -109.4 & -83.5 & no dock & -90.1 & -94.7 \\
\hline Physagulin L' & -107.4 & -90.3 & no dock & -102.4 & -101.4 \\
\hline Physagulin M & -106.5 & -78.5 & no dock & -95.7 & -94.4 \\
\hline Physagulin $\mathrm{M}^{\prime}$ & -113.3 & -81.6 & no dock & -94.8 & -91.5 \\
\hline Physagulin N & -114.0 & -91.4 & no dock & -92.6 & -95.2 \\
\hline Physagulin $\mathrm{N}^{\prime}$ & -100.5 & -87.7 & no dock & -85.7 & -106.2 \\
\hline Physagulin O & -108.2 & -77.9 & no dock & -94.4 & -92.2 \\
\hline Physalin A & -105.0 & -75.2 & no dock & -81.4 & -73.9 \\
\hline Physalin B & -110.4 & -72.6 & no dock & -86.0 & -93.8 \\
\hline Physalin D & -113.5 & -86.0 & no dock & -69.0 & -93.0 \\
\hline Physalin E & -115.4 & -72.5 & no dock & -82.9 & -92.6 \\
\hline Physalin F & -111.9 & -73.6 & no dock & -76.2 & -93.0 \\
\hline Physalin G & -99.1 & -69.1 & no dock & -77.5 & -91.0 \\
\hline Physalin H & -112.4 & -83.7 & no dock & -70.2 & -94.5 \\
\hline Physalin I & -101.3 & -72.0 & no dock & -72.8 & -94.2 \\
\hline Physalin J & -111.1 & -83.9 & -34.5 & -91.4 & -93.0 \\
\hline Physalin K & -93.9 & -57.4 & no dock & -70.4 & -89.5 \\
\hline Physalin U & -99.8 & -80.0 & -37.3 & -78.6 & -94.1 \\
\hline Physalin V & -114.5 & -67.3 & -50.4 & -76.0 & -102.3 \\
\hline Physalin W & -98.6 & -76.3 & no dock & -71.1 & -81.1 \\
\hline Physangulide & -116.5 & -94.1 & no dock & -103.3 & -103.2 \\
\hline Physanolide A & -104.8 & -90.5 & no dock & -88.1 & -95.2 \\
\hline Vamonolide & -105.8 & -78.1 & no dock & -88.6 & -97.3 \\
\hline Withangulatin A & -114.5 & -92.1 & no dock & -98.6 & -94.2 \\
\hline Withangulatin B & -112.3 & -83.6 & no dock & -93.2 & -90.6 \\
\hline Withangulatin C & -112.1 & -77.3 & no dock & -89.5 & -87.7 \\
\hline Withangulatin D & -106.6 & -75.7 & no dock & -79.5 & -96.3 \\
\hline Withangulatin E & -120.7 & -82.1 & no dock & -89.9 & -85.6 \\
\hline Withangulatin F & -111.9 & -80.4 & no dock & -93.8 & -92.6 \\
\hline Withangulatin $\mathrm{G}$ & -115.5 & -75.1 & no dock & -77.0 & -97.7 \\
\hline Withangulatin $\mathrm{H}$ & -119.1 & -74.0 & -40.1 & -103.9 & -89.9 \\
\hline Withangulatin I & -118.5 & -92.6 & no dock & -98.5 & -97.6 \\
\hline
\end{tabular}


Table 35. MolDock docking energies $(\mathrm{kJ} / \mathrm{mol})$ of triterpenoids with Leishmania major protein targets.

\begin{tabular}{|c|c|c|c|c|c|c|c|c|c|c|c|c|}
\hline Triterpenoids & LmajCatB & LmajDHODH & LmajdUTPase & LmajNDKb & LmajNH & LmajNMT & LmajOPB & LmajPDE1 & LmajPTR1 & LmajMetRS & LmajTyrRS & LmajUGPase \\
\hline$\alpha-$ Amyrin & -74.4 & -81.7 & -52.9 & -55.1 & -73.4 & -84.8 & -75.6 & -100.9 & -72.3 & -71.8 & -84.5 & -82.7 \\
\hline$\beta$-Amyrin & -43.0 & -78.8 & -66.1 & -59.1 & -76.8 & -87.7 & -84.4 & -83.8 & -65.7 & -72.6 & -84.0 & -82.4 \\
\hline Betulin & -71.9 & -85.3 & -75.8 & -61.5 & -99.0 & -93.6 & -94.4 & -83.0 & -71.2 & -80.8 & -99.1 & -85.5 \\
\hline Betulinaldehyde & -74.6 & -81.7 & -75.7 & -71.9 & -98.9 & -93.5 & -94.2 & -88.4 & -76.0 & -78.5 & -99.1 & -89.4 \\
\hline Betulinic acid & -74.2 & -88.5 & -66.7 & -61.3 & -96.3 & -104.6 & -89.1 & -82.6 & -73.5 & -82.0 & -103.3 & -88.7 \\
\hline Corosolic acid & -80.2 & -74.7 & -81.7 & -63.1 & -91.3 & -107.7 & -84.5 & -106.4 & -81.6 & -79.3 & -86.4 & -81.4 \\
\hline Erythrodiol & -34.6 & -84.6 & -64.6 & -64.9 & -79.5 & -92.7 & -87.0 & -84.9 & -67.2 & -74.7 & -87.6 & -83.5 \\
\hline Friedelin & -57.3 & -72.5 & -74.6 & -53.9 & -84.9 & -75.9 & -78.0 & -79.6 & -75.9 & -71.3 & -102.4 & -76.5 \\
\hline Isoiguesterin & -67.3 & -79.4 & -64.7 & -73.5 & -80.5 & -95.5 & -81.0 & -92.2 & -77.3 & -77.2 & -88.2 & -85.2 \\
\hline 20-epi-Isoiguesterinol & -69.7 & -74.0 & -64.7 & -83.9 & -85.3 & -96.4 & -98.2 & -96.2 & -84.4 & -81.4 & -83.6 & -78.6 \\
\hline Lawnermis acid methyl ester & -75.8 & -95.9 & -81.6 & -70.3 & -76.5 & -91.4 & -81.6 & -98.3 & -66.7 & -78.8 & -92.2 & -88.3 \\
\hline Lupeol & -73.2 & -84.6 & -76.7 & -53.1 & -99.0 & -87.5 & -91.5 & -85.5 & -69.9 & -74.3 & -96.2 & -75.9 \\
\hline Methyl seco-3,4-betulonic acid & -87.2 & -93.8 & -84.8 & -76.1 & -101.2 & -113.5 & -96.5 & -106.3 & -97.0 & -79.2 & -90.1 & -110.1 \\
\hline 3-O-Methyl-6-oxopristimerol & -91.4 & -92.8 & -75.9 & -77.1 & -99.5 & -102.5 & -88.4 & -95.2 & -79.1 & -94.0 & -99.9 & -96.1 \\
\hline Oleanolic acid & -82.5 & -90.6 & -80.2 & -66.3 & -72.7 & -97.7 & -79.8 & -95.3 & -66.6 & -69.7 & -88.4 & -76.2 \\
\hline epi-Oleanolic acid & -75.9 & -76.7 & -77.2 & -54.2 & -72.4 & -83.7 & -88.7 & -98.5 & -70.3 & -84.2 & -82.3 & -95.6 \\
\hline 6-Oxopristimerol & -87.2 & -91.5 & -72.9 & -76.4 & -98.8 & -103.6 & -90.2 & -91.8 & -82.5 & -93.2 & -93.8 & -101.2 \\
\hline $\begin{array}{l}(24 Z)-3-\text { Oxotirucalla-7,24-dien- } \\
26-\text { oic acid }\end{array}$ & -96.4 & -95.4 & -86.1 & -129.2 & -104.4 & -95.7 & -99.7 & -106.1 & -90.3 & -91.9 & -102.2 & -98.9 \\
\hline Pristimerin & -69.3 & -87.8 & -72.3 & -77.6 & -88.1 & -112.9 & -86.3 & -86.4 & -87.8 & -86.6 & -102.0 & -88.6 \\
\hline Rotundic acid & -78.5 & -85.1 & -74.2 & -49.1 & -55.9 & -85.7 & -86.8 & -98.4 & -65.6 & -80.8 & -78.5 & -83.4 \\
\hline Taraxerol & -22.5 & -83.4 & -60.2 & -48.0 & -70.1 & -82.4 & -88.0 & -78.8 & -85.5 & -85.1 & -84.9 & -88.1 \\
\hline Ursolic acid & -70.8 & -80.0 & -74.0 & -59.1 & -72.0 & -92.4 & -80.4 & -73.7 & -71.9 & -82.7 & -84.3 & -89.9 \\
\hline Uvaol & -74.9 & -82.4 & -67.9 & -67.8 & -75.2 & -88.3 & -85.4 & -89.9 & -75.2 & -74.9 & -86.4 & -87.0 \\
\hline Wallichianol & -81.9 & -93.2 & -71.1 & -74.6 & -87.2 & -102.7 & -92.0 & -75.2 & -86.2 & -80.1 & -80.6 & -89.4 \\
\hline
\end{tabular}


Table 36. MolDock docking energies (kJ/mol) of triterpenoids with Leishmania donovani and L. mexicana protein targets.

\begin{tabular}{|c|c|c|c|c|c|c|c|c|c|c|c|c|}
\hline Triterpenoids & LdonCatB & LdonCyp & LdonDHODH & LdonNMT & LmexGAPDH & LmexGPDH & LmexPGI & LmexPMM & $\begin{array}{c}\text { LmexPYK } \\
\text { Site } 1 \\
\end{array}$ & $\begin{array}{c}\text { LmexPYK } \\
\text { Site } 2 \\
\end{array}$ & $\begin{array}{c}\text { LmexPYK } \\
\text { Site } 3 \\
\end{array}$ & LmexTIM \\
\hline$\alpha$-Amyrin & -79.7 & -79.4 & -19.0 & -53.4 & -73.7 & -88.0 & -73.5 & -71.7 & -70.4 & -87.4 & -85.4 & -54.4 \\
\hline$\beta$-Amyrin & -65.3 & -75.6 & no dock & -64.7 & -73.9 & -77.2 & -65.3 & -71.0 & -79.9 & -94.4 & -75.2 & -35.5 \\
\hline Betulin & -80.1 & -78.2 & no dock & -77.8 & -81.6 & -101.1 & -79.1 & -82.6 & -87.1 & -98.0 & -83.7 & -70.3 \\
\hline Betulinaldehyde & -82.8 & -78.6 & no dock & -67.2 & -75.9 & -96.9 & -83.5 & -84.5 & -87.5 & -96.6 & -82.5 & -75.0 \\
\hline Betulinic acid & -86.3 & -65.2 & no dock & -80.7 & -77.7 & -97.3 & -81.0 & -87.8 & -89.5 & -97.8 & -85.4 & -76.3 \\
\hline Corosolic acid & -80.8 & -80.2 & no dock & -78.2 & -78.0 & -93.2 & -81.9 & -75.8 & -80.7 & -89.6 & -73.0 & -60.9 \\
\hline Erythrodiol & -57.7 & -75.8 & -30.0 & -69.5 & -76.1 & -80.8 & -69.9 & -73.0 & -79.2 & -96.6 & -75.4 & -39.3 \\
\hline Friedelin & -65.9 & -53.9 & -48.8 & -63.2 & -72.7 & -83.8 & -64.8 & -77.1 & -73.6 & -86.1 & -76.7 & -70.1 \\
\hline Isoiguesterin & -69.9 & -70.0 & -48.8 & -70.4 & -76.6 & -80.9 & -68.8 & -83.0 & -80.7 & -87.9 & -77.0 & -65.9 \\
\hline 20-epi-Isoiguesterinol & -70.5 & -83.0 & -55.8 & -74.8 & -75.9 & -93.0 & -73.1 & -91.0 & -88.9 & -88.7 & -76.3 & -58.3 \\
\hline Lawnermis acid methyl ester & -73.7 & -77.4 & -44.5 & -73.4 & -84.7 & -97.0 & -80.1 & -77.6 & -81.5 & -96.3 & -98.1 & -57.7 \\
\hline Lupeol & -80.7 & -76.5 & no dock & -71.9 & -88.5 & -93.0 & -72.1 & -81.3 & -82.0 & -96.0 & -79.6 & -69.2 \\
\hline Methyl seco-3,4-betulonic acid & -91.6 & -83.9 & no dock & -92.0 & -87.8 & -102.4 & -89.7 & -98.0 & -109.4 & -102.6 & -86.7 & -82.2 \\
\hline 3-O-Methyl-6-oxopristimerol & -90.8 & -83.7 & no dock & -85.0 & -76.0 & -90.2 & -78.6 & -92.9 & -109.5 & -96.4 & -83.2 & -61.4 \\
\hline Oleanolic acid & -82.4 & -74.2 & no dock & -69.7 & -79.0 & -86.9 & -69.9 & -80.8 & -77.5 & -90.4 & -79.9 & -74.9 \\
\hline epi-Oleanolic acid & -61.1 & -73.7 & -50.7 & -69.1 & -81.2 & -88.8 & -66.3 & -78.6 & -79.6 & -91.9 & -74.8 & -65.7 \\
\hline 6-Oxopristimerol & -90.6 & -84.3 & -60.9 & -82.9 & -80.8 & -90.0 & -79.1 & -85.5 & -98.4 & -95.2 & -94.1 & -68.0 \\
\hline (24Z)-3-Oxotirucalla-7,24-dien-26-oic acid & -100.0 & -88.1 & -82.2 & -89.0 & -94.3 & -108.5 & -99.2 & -104.0 & -122.1 & -104.4 & -100.1 & -91.2 \\
\hline Pristimerin & -71.3 & -78.4 & -46.7 & -85.9 & -93.1 & -90.7 & -74.4 & -102.4 & -96.4 & -97.4 & -80.4 & -63.0 \\
\hline Rotundic acid & -71.6 & -78.2 & -36.3 & -77.0 & -75.8 & -100.3 & -71.4 & -75.5 & -80.8 & -93.7 & -82.5 & -58.0 \\
\hline Taraxerol & -51.7 & -78.0 & -29.6 & -61.4 & -69.4 & -82.8 & -68.2 & -90.4 & -81.4 & -90.1 & -69.8 & -81.4 \\
\hline Ursolic acid & -69.6 & -78.7 & no dock & -71.7 & -76.5 & -97.3 & -80.3 & -75.5 & -71.8 & -93.0 & -75.4 & -46.5 \\
\hline Uvaol & -81.4 & -79.1 & no dock & -51.7 & -75.9 & -95.0 & -78.0 & -74.3 & -76.4 & -92.0 & -82.8 & -64.9 \\
\hline Wallichianol & -81.5 & -70.6 & -58.4 & -85.4 & -83.8 & -87.6 & -80.4 & -91.9 & -96.8 & -93.8 & -82.3 & -77.4 \\
\hline
\end{tabular}


Table 37. MolDock docking energies $(\mathrm{kJ} / \mathrm{mol})$ of triterpenoids with Leishmania infantum protein targets.

\begin{tabular}{lccccc}
\hline Triterpenoids & LinfCYP51 & LinfGLO2 & LinfPnC1 & LinfTDR1 & LinfTR \\
\hline$\alpha-$ Amyrin & -99.3 & -66.0 & no dock & -66.7 & -71.9 \\
$\beta-$ Amyrin & -98.9 & -72.6 & -43.9 & -73.5 & -68.4 \\
Betulin & -101.6 & -73.8 & -30.4 & -79.1 & -79.0 \\
Betulinaldehyde & -104.8 & -74.8 & no dock & -74.1 & -85.8 \\
Betulinic acid & -95.5 & -75.2 & no dock & -80.5 & -85.8 \\
Corosolic acid & -111.8 & -64.5 & no dock & -74.2 & -82.2 \\
Erythrodiol & -100.4 & -71.2 & no dock & -76.7 & -78.9 \\
Friedelin & -92.7 & -64.4 & -40.3 & -71.0 & -75.4 \\
Isoiguesterin & -83.7 & -70.6 & no dock & -81.9 & -77.2 \\
20-epi-Isoiguesterinol & -81.1 & -71.1 & no dock & -80.4 & -77.1 \\
Lawnermis acid methyl ester & -97.9 & -72.9 & -33.4 & -72.3 & -81.2 \\
Lupeol & -97.9 & -70.7 & -35.1 & -77.1 & -81.0 \\
Methyl seco-3,4-betulonic acid & -94.7 & -67.1 & no dock & -88.7 & -91.9 \\
3-O-Methyl-6-oxopristimerol & -98.6 & -73.3 & -12.0 & -84.9 & -92.2 \\
Oleanolic acid & -105.6 & -70.7 & no dock & -79.2 & -74.9 \\
epi-Oleanolic acid & -105.5 & -74.4 & no dock & -81.6 & -80.5 \\
6-Oxopristimerol & -95.8 & -66.4 & no dock & -77.6 & -94.9 \\
(24Z)-3-Oxotirucalla-7, & -112.4 & -99.4 & no dock & -83.9 & -97.0 \\
24-dien-26-oic acid & -97.5 & -78.3 & no dock & -82.4 & -84.9 \\
Pristimerin & -107.8 & -60.5 & -33.1 & -76.5 & -78.6 \\
Rotundic acid & -91.5 & -74.2 & -39.1 & -74.8 & -76.9 \\
Taraxerol & -102.3 & -62.5 & no dock & -69.4 & -76.3 \\
Ursolic acid & -101.1 & -65.3 & no dock & -69.4 & -77.4 \\
Uvaol & & & & & \\
\hline & & & &
\end{tabular}


Table 38. MolDock docking energies $(\mathrm{kJ} / \mathrm{mol})$ of quassinoids and steroids with Leishmania major protein targets.

\begin{tabular}{|c|c|c|c|c|c|c|c|c|c|c|c|c|}
\hline \multicolumn{13}{|l|}{ Quassinoids } \\
\hline $15-\beta$-Heptylchaparrinone & -92.7 & -121.0 & -89.4 & -118.4 & -101.9 & -112.4 & -98.2 & -109.3 & -96.2 & -120.5 & -106.5 & -101.0 \\
\hline Simalikalactone D & -88.4 & -106.2 & -80.5 & -99.3 & -84.9 & -98.5 & -63.6 & -105.2 & -91.8 & -111.7 & -105.5 & -111.1 \\
\hline \multicolumn{13}{|l|}{ Steroids } \\
\hline Cholesterol & -90.5 & -95.2 & -93.3 & -106.4 & -101.7 & -98.6 & -111.4 & -109.2 & -109.5 & -115.2 & -99.3 & -97.7 \\
\hline Clerosterol & -97.9 & -100.6 & -95.5 & -110.6 & -104.8 & -102.4 & -91.6 & -113.3 & -110.8 & -121.3 & -101.0 & -101.9 \\
\hline 24-Hydroperoxy-24-vinylcholesterol & -81.0 & -99.9 & -92.1 & -111.0 & -103.4 & -105.0 & -115.9 & -117.7 & -109.8 & -127.0 & -100.2 & -112.4 \\
\hline Lanosterol & -85.0 & -85.4 & -81.3 & -94.1 & -103.2 & -96.3 & -111.4 & -108.4 & -87.2 & -88.8 & -97.2 & -99.3 \\
\hline Saringosterol & -88.4 & -103.8 & -89.8 & -108.1 & -105.9 & -101.6 & -109.5 & -115.5 & -107.1 & -126.4 & -99.0 & -106.1 \\
\hline$\beta$-Sitosterol & -94.8 & -98.7 & -94.8 & -106.4 & -105.6 & -102.1 & -101.7 & -111.9 & -110.0 & -121.5 & -102.2 & -111.6 \\
\hline Stigmasterol & -87.8 & -102.3 & -94.6 & -105.6 & -108.1 & -101.1 & -101.0 & -109.8 & -109.3 & -121.4 & -106.4 & -102.1 \\
\hline
\end{tabular}

Table 39. MolDock docking energies $(\mathrm{kJ} / \mathrm{mol})$ of quassinoids and steroids with Leishmania donovani and L. mexicana protein targets.

\begin{tabular}{|c|c|c|c|c|c|c|c|c|c|c|c|c|}
\hline Quassinoids & LdonCatB & LdonCyp & LdonDHODH & LdonNMT & LmexGAPDH & LmexGPDH & LmexPGI & LmexPMM & $\begin{array}{c}\text { LmexPYK } \\
\text { Site } 1 \\
\end{array}$ & $\begin{array}{c}\text { LmexPYK } \\
\text { Site } 2 \\
\end{array}$ & $\begin{array}{c}\text { LmexPYK } \\
\text { Site } 3 \\
\end{array}$ & LmexTIM \\
\hline $15-\beta-$ Heptylchaparrinone & -93.0 & -90.0 & -88.8 & -100.5 & -84.9 & -111.8 & -90.5 & -110.0 & -123.2 & -108.0 & -98.8 & -92.0 \\
\hline Simalikalactone D & -98.1 & -102.9 & no dock & -90.9 & -89.5 & -115.2 & -70.9 & -103.9 & -93.4 & -99.6 & -107.4 & -75.8 \\
\hline \multicolumn{13}{|l|}{ Steroids } \\
\hline Cholesterol & -95.4 & -99.4 & -78.2 & -90.9 & -91.6 & -102.8 & -93.8 & -104.3 & -102.0 & -95.5 & -100.2 & -87.3 \\
\hline Clerosterol & -82.5 & -99.9 & -89.1 & -99.9 & -92.8 & -104.0 & -80.0 & -108.3 & -106.8 & -104.2 & -100.1 & -96.0 \\
\hline 24-Hydroperoxy-24-vinylcholesterol & -97.8 & -98.0 & -101.7 & -89.9 & -93.5 & -97.2 & -77.0 & -111.9 & -103.8 & -104.4 & -89.5 & -91.6 \\
\hline Lanosterol & -84.1 & -88.4 & -55.0 & -81.9 & -81.1 & -99.7 & -91.5 & -98.8 & -103.6 & -93.5 & -103.8 & -82.4 \\
\hline Saringosterol & -97.9 & -106.5 & -98.9 & -99.1 & -86.7 & -113.8 & -91.1 & -111.0 & -106.5 & -102.3 & -88.7 & -95.8 \\
\hline$\beta$-Sitosterol & -100.9 & -104.5 & -86.7 & -88.8 & -92.5 & -100.0 & -96.7 & -107.8 & -105.5 & -97.3 & -92.6 & -95.0 \\
\hline Stigmasterol & -97.0 & -98.5 & -93.7 & -92.3 & -90.2 & -112.3 & -88.5 & -107.6 & -106.0 & -104.3 & -97.8 & -96.5 \\
\hline
\end{tabular}


Table 40. MolDock docking energies $(\mathrm{kJ} / \mathrm{mol})$ of quassinoids and steroids with Leishmania infantum protein targets.

\begin{tabular}{lccccc}
\hline Quassinoids & LinfCYP51 & LinfGLO2 & LinfPnC1 & LinfTDR1 & LinfTR \\
\hline 15- $\beta-$ Heptylchaparrinone & -107.6 & -100.4 & no dock & -92.2 & -96.8 \\
Simalikalactone D & -98.5 & -85.8 & no dock & -83.7 & -80.2 \\
\hline Steroids & & & & & \\
\hline Cholesterol & -110.9 & -86.9 & no dock & -87.4 & -93.0 \\
Clerosterol & -116.3 & -94.5 & no dock & -91.4 & -95.8 \\
24-Hydroperoxy-24-vinylcholesterol & -121.5 & -89.9 & no dock & -91.4 & -96.6 \\
Lanosterol & -116.0 & -85.7 & no dock & -81.5 & -90.4 \\
Saringosterol & -117.2 & -90.0 & no dock & -97.9 & -99.8 \\
$\beta-$ Sitosterol & -113.8 & -87.6 & no dock & -94.1 & -103.0 \\
Stigmasterol & -116.1 & -86.4 & no dock & -96.6 & -98.4 \\
\hline
\end{tabular}

Not surprisingly, all of the steroids and many of the triterpenoids examined in this study showed significant docking preference for L. infantum sterol 14 $\alpha$-demethylase (LinfCYP51). This had been

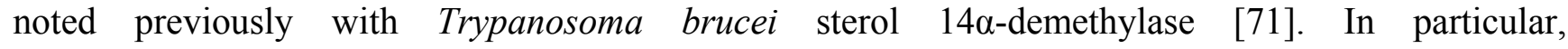
24-hydroperoxy-24-vinylcholesterol (docking energy $=-121.5 \mathrm{~kJ} / \mathrm{mol}$ ) and 24,25-epoxywithanolide D (docking energy $=-128.1 \mathrm{~kJ} / \mathrm{mol}$ ) were strongly docking with LinfCYP51. The lowest-energy pose of 24-hydroperoxy-24,25-vinylcholesterol with LinfCYP51 places the hydroperoxy group of the ligand adjacent to the heme Fe (Figure 28); this ligand, then, can presumably oxidize the Fe and render the enzyme inactive.

Figure 25. Lowest-energy docked pose of grandifontane with L. major dihydroorotate dehydrogenase (LmajDHODH, PDB 3mhu) showing key interactions with Cys 131, Asn 199, Asn 68, Ser 69, and Gln 139. Hydrogen-bonds are shown as blue dashed lines.

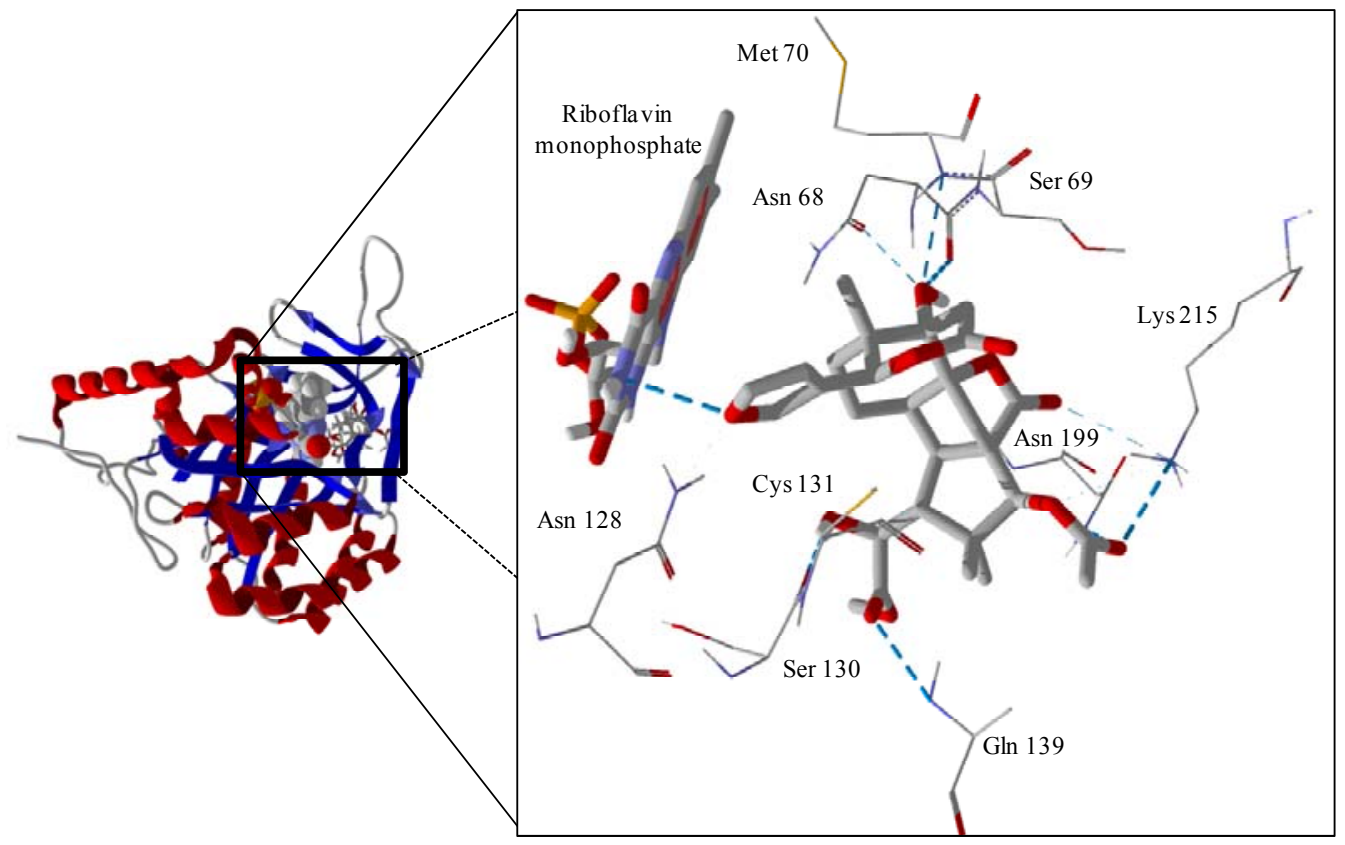


Figure 26. Lowest-energy docked pose of 6-O-acetylswietenolide with $\mathrm{L}$. mexicana glycerol-3-phosphate dehydrogenase (LmexGPDH, PDB 1n1e) showing key interactions with Arg 274, Ser 293, Phe 26 and Ala 157, and Gln 139. Hydrogen-bonds are shown as blue dashed lines.

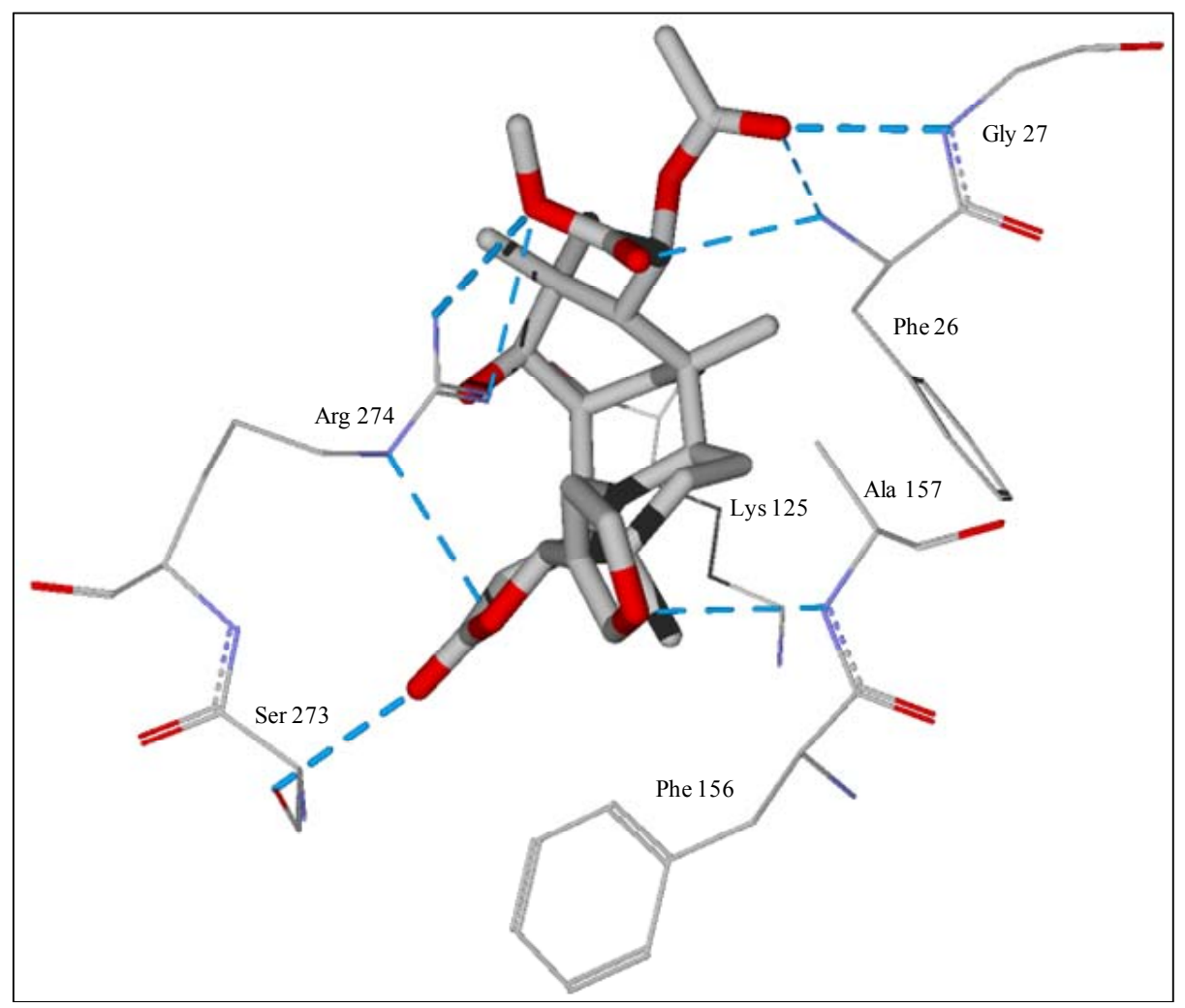

Figure 27. Lowest-energy poses of steroids, cholesterol (white), clerosterol (purple), saringosterol (green), stigmasterol (cyan), $\beta$-sitosterol (blue), and 24-hydroperoxy-24vinylcholesterol (red), in the hydropobic pocket of $L$. major methionyl t-RNA synthetase (LmajMetRS, PDB 3kfl).

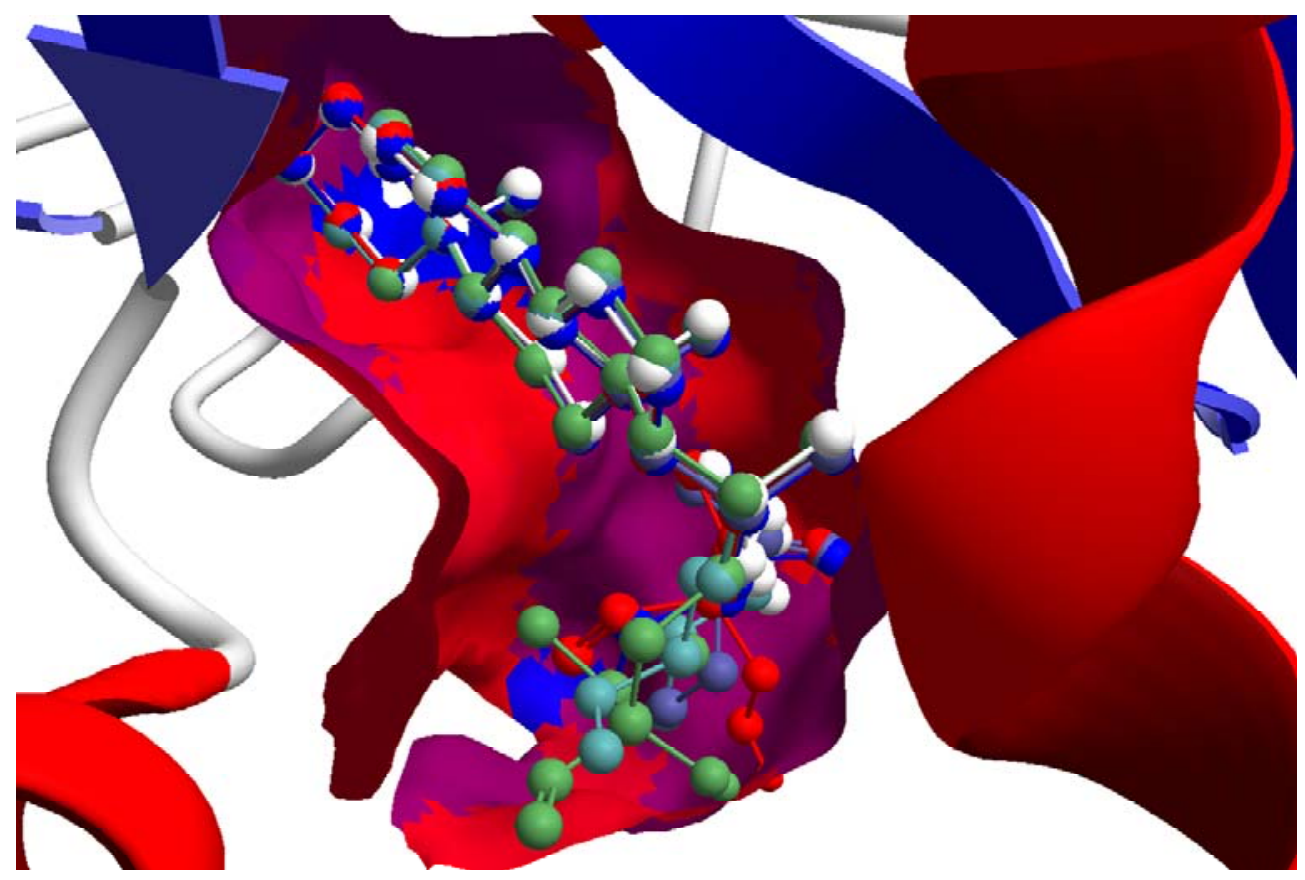


Figure 28. Lowest-energy poses of 24-hydroperoxy-24-vinylcholesterol with $L$. infantum sterol 14 $\alpha$-demethylase (LinfCYP51, PDB 314d). Note the proximity of the hydroperoxy group of the ligand with the Fe atom of the heme cofactor.

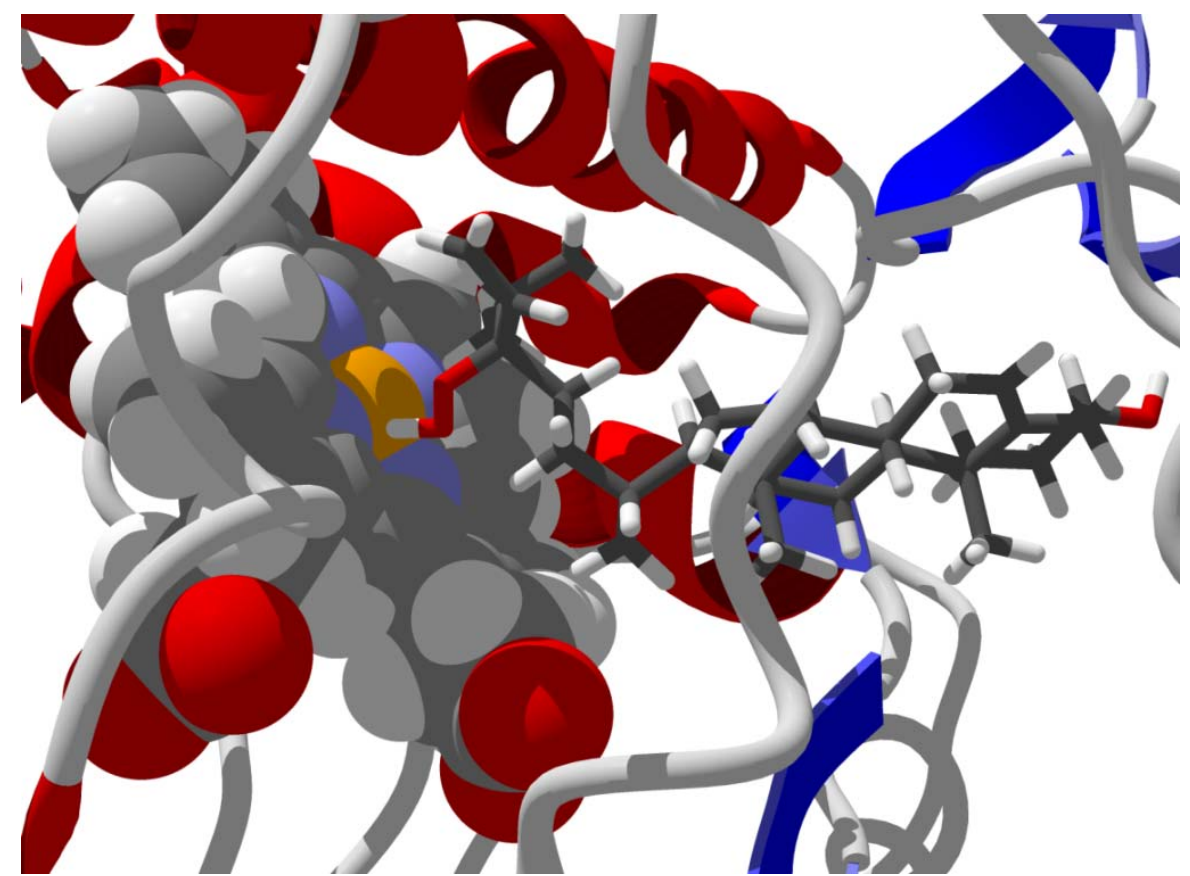

An examination of docking energies with respect to ligand molecular size suggests that for terpenoid ligands there is a threshold where larger size does not correspond to stronger binding to the protein target. A plot of molecular weights of representative terpenoids (monoterpenoids, germacranolide sesquiterpenoids, labdane diterpenoids, and triterpenoids) and docking energies to three different protein targets (LmajMetRS, LmexGPDH, and LdonCyp) (Figure 29) shows that strongest docking energies are terpenoids with molecular weights around 360-430 amu.

\section{Computational Methods}

Protein-ligand docking studies were carried out based on the crystal structures of verified Leishmania protein drug targets: L. major cathepsin B, LmajCatB (prepared by structural homology to Trypanosoma brucei cathepsin B, PDB 3hhi [72]), L. major dihydroorotate dehydrogenase, LmajDHODH (PDB 3gye [73], PDB 3mhu, and PDB 3mjy [74]), L. major methionyl-tRNA synthetase, LmajMetRS (PDB 3kfl [51]), L. major nucleoside diphosphate kinase b, LmajNDKb (PDB 3ngs, PDB 3ngt, and PDB 3ngu [38]), L. major nucleoside hydrolase, LmajNH (PDB 1ezr [35]), L. major $N$-myristoyltransferase, LmajNMT (PDB 2wsa, PDB 3h5z [47], and PDB 4a30 [75]), L. major oligopeptidase B, LmajOPB (PDB 2xe4 [27]), L. major phosphodiesterase 1, LmajPDE1 (PDB 2r8q [40]), L. major pteridine reductase 1, LmajPTR1 (PDB 1e7w [43], PDB 1w0c [76], PDB 2 bf7 [77], and PDB 3h4v [78]), L. major tyrosyl-tRNA synthetase, LmajTyrRS (PDB 3p0h and PDB 3p0j [52]), L. major uridine diphosphate-glucose pyrophosphorylase, LmajUGPase (PDB 2oef and PDB 2oeg [10]), L. major deoxyuridine triphosphate nucleotidohydrolase, LmajdUTPase (PDB 2yay and PDB $2 \mathrm{yb0}$ [33]), L. donovani cathepsin B, LdonCatB (prepared by structural homology to T. brucei cathepsin B, PDB 3hhi [72]), L. donovani cyclophilin, LdonCyp (PDB 2haq [49] and PDB 
3eov [79]), L. donovani dihydroorotate dehydrogenase, LdonDHODH (PDB 3c61 [80]), L. donovani $N$-myristoyltransferase, LdonNMT (PDB 2wuu [48]), L. mexicana glyceraldehyde-3-phosphate dehydrogenase. LmexGAPDH (PDB 1a7k [11] and PDB 1gyp [81]), L. mexicana glycerol-3phosphate dehydrogenase, LmexGPDH (PDB 1evz [14], PDB 1m66, PDB 1n1e and PDB 1n1g [82]), L. mexicana phosphoglucose isomerase, LmexPGI (PDB 1q50 and PDB 1t10 [8]), L. mexicana phosphomannomutase, LmexPMM (PDB $2 \mathrm{i} 54$ and PDB $2 \mathrm{i} 55$ [83]), L. mexicana pyruvate kinase, LmexPYK (PDB 1pkl [6], PDB 3hqp [84], and PDB 3pp7 [85]), L. mexicana triosephosphate isomerase, LmexTIM (PDB 2vxn [17] and PDB 2y61 [86]), L. infantum sterol 14 $\alpha$-demethylase, LinfCYP51 (PDB 314d [87]), L. infantum glyoxalase II, LinfGLO2 (PDB 2p1e and PDB 2p18 [21]), L. infantum nicotinamidase, LinfPnC1 (PDB 3r2j [34]), L. infantum thiol-dependent reductase I, LinfTDR1 (PDB 4ags [19]), and L. infantum trypanothione reductase, LinfTR (PDB 2yau [88] and PDB 4adw [89] and PDB 4apn [90]) Prior to docking all solvent molecules and the co-crystallized ligands were removed from the structures. Molecular docking calculations for all compounds with each of the proteins were undertaken using Molegro Virtual Docker v. 5.0 [91,92], with a sphere large enough to accommodate the cavity centered on the binding sites of each protein structure in order to allow each ligand to search. If a co-crystallized inhibitor or substrate was present in the structure, then that site was chosen as the binding site. If no co-crystallized ligand was present, then suitably sized cavities were used as potential binding sites. Standard protonation states of the proteins based on neutral $\mathrm{pH}$ were used in the docking studies. The protein was used as a rigid model structure; no relaxation of the protein was performed. Assignments of charges on each protein were based on standard templates as part of the Molegro Virtual Docker program; no other charges were necessary to be set. Each ligand structure was built using Spartan '10 for Windows [93]. The structures were geometry optimized using the MMFF force field [94]. Flexible ligand models were used in the docking and subsequent optimization scheme. As a test of docking accuracy and for docking energy comparison, co-crystallized ligands were re-docked into the protein structures (See Table 41). Different orientations of the ligands were searched and ranked based on their energy scores. The RMSD threshold for multiple cluster poses was set at $<1.00 \AA$. The docking algorithm was set at maximum iterations of 1500 with a simplex evolution population size of 50 and a minimum of 30 runs for each ligand. Each binding site of oligomeric structures was searched with each ligand. The lowestenergy (strongest-docking) poses for each ligand in each protein target are summarized in Tables 1-28.

The primary sequence of the cathepsin B-like cysteine protease from T. brucei (TbCatB, PDB 3 hhi [72]) was compared to the query sequences of the same functional enzyme from L. donovani (LdonCatB) and L. major (LmajCatB) using the Protein BLAST (Basic Local Alignment Search Tool). Regions of local similarity and identity were found between the query sequences of LdonCatB and LmajCatB when compared to the model sequence of TbCatB. Both LdonCatB and LmajCatB sequences had $52 \%$ and $54 \%$ identity with that of $\mathrm{TbCatB}$, respectively. The three-dimensional structure of TbCatB has been determined to $1.6 \AA$ (PDB 3hhi [72]) but there is no structural information available for either LdonCatB or LmajCatB. Calculated models for both LdonCatB and LmajCatB were obtained from combining sequence information of the unknown target structures for LdonCatB and LmajCatB with the known model of TbCatB. The alignment between target and model sequences was used to modify the model PDB TbCatB file by pruning non-conserved residues to the last common atoms using the CCP4 chainsaw molecular replacement utility $[95,96]$ leaving conserved 
residues unchanged. The resulting models were refined with conjugate gradient minimization with no experimental energy terms used in the crystallographic and NMR System (CNS) program suite [97]. The resulting detailed model was refined with conjugate gradient minimization with no experimental energy terms used. All atoms of the molecules were unrestrained and were minimized for 500 steps with a continuous dielectric constant of one.

Figure 29. Plots of docking energies $v s$. molecular weights for representative isoprenoids with three Leishmania protein targets.

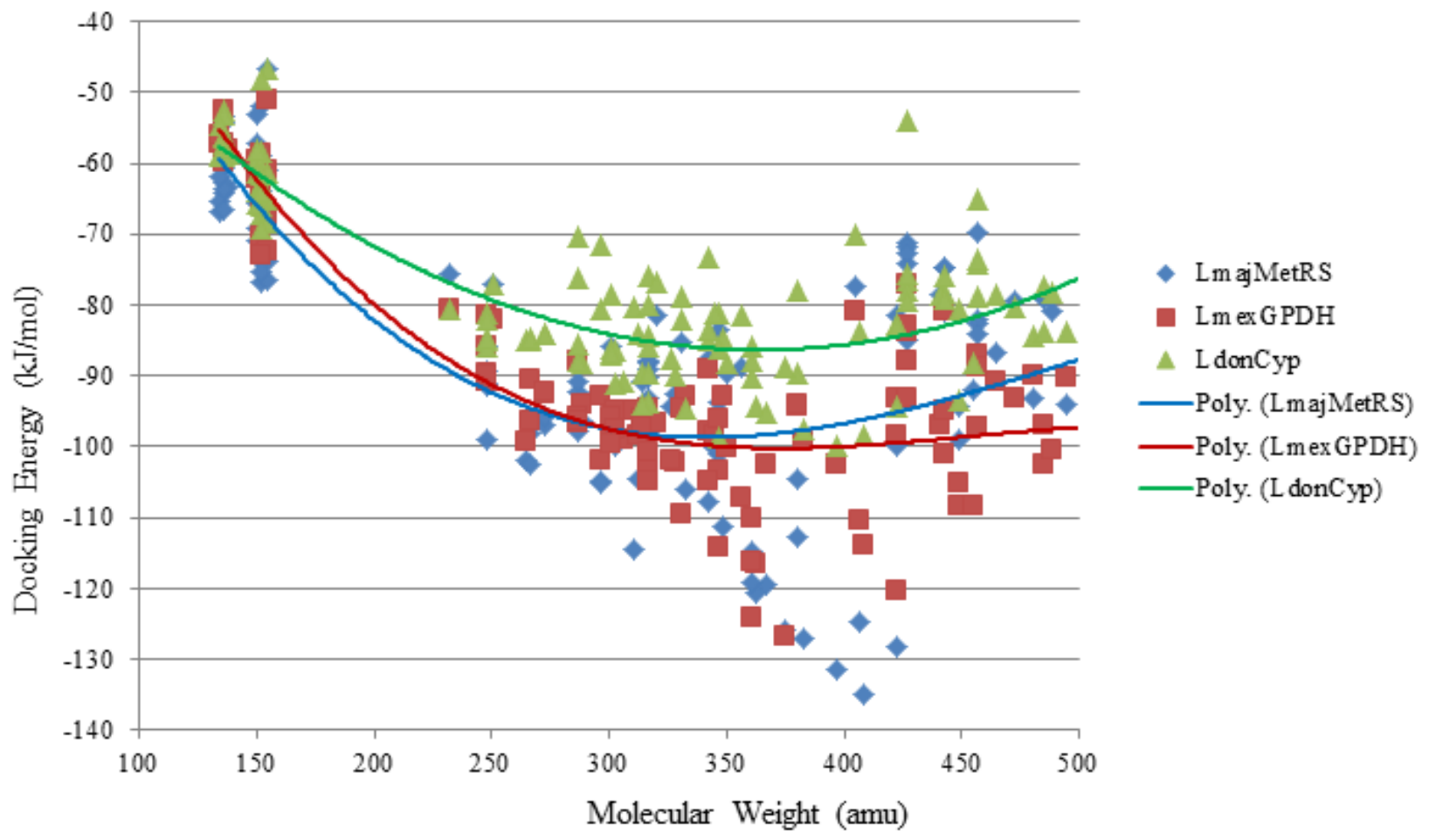


Table 41. MolDock docking energies of co-crystallized ligands and root-mean-squared deviations between the co-crystallized ligand and the re-docked poses of the co-crystallized ligand with Leishmania protein crystal structures.

\begin{tabular}{|c|c|c|c|c|}
\hline Protein Target & PDB code & Co-crystallized ligand & $E(\mathbf{k J} / \mathbf{m o l})$ & $\operatorname{RMSD}(\AA ̊)$ \\
\hline LmajCatB & homology & none & --- & \\
\hline \multirow[t]{3}{*}{ LmajDHODH } & 3gye & none & --- & \\
\hline & $3 \mathrm{mhu}$ & 5-nitroorotic acid & -102.2 & 0.37 \\
\hline & 3 mjy & 5-aminoorotic acid & -91.4 & 0.47 \\
\hline \multirow[t]{2}{*}{ LmajdUTPase } & 2yay & $2^{\prime}$-deoxyuridine- 5 '- $\alpha, \beta$-imido-triphosphate & -117.1 & 1.36 \\
\hline & $2 \mathrm{yb} 0$ & 2'-deoxyuridine & -80.3 & 0.93 \\
\hline \multirow[t]{3}{*}{ LmajNDKb } & 3 ngs & none & --- & \\
\hline & 3 ngt & adenosine 5'-monophosphate & -122.5 & 4.07 \\
\hline & 3ngu & adenosine 5 '-diphosphate & -143.6 & 4.29 \\
\hline LmajNH & lezr & none & --- & \\
\hline \multirow[t]{3}{*}{ LmajNMT } & 2 wsa & $\begin{array}{l}\text { 2,6-dichloro-4-(2-piperazin-1-ylpyridin-4- yl)- } N-(1,3,5- \\
\text { trimethyl-1H-pyrazol-4-yl)benzenesulfonamide }\end{array}$ & -121.0 & 0.88 \\
\hline & $3 h 5 z$ & myristoyl-CoA & -115.4 & 7.52 \\
\hline & $4 \mathrm{a} 30$ & $\begin{array}{l}\text { 4-bromo-2,6-dichloro- } N \text {-(1,3,5-trimethyl-1H-pyrazol-4- } \\
\text { yl)benzene-sulfonamide }\end{array}$ & -84.5 & 1.80 \\
\hline LmajOPB & $2 \mathrm{xe} 4$ & none & --- & \\
\hline LmajPDE1 & $2 \mathrm{r} 8 \mathrm{q}$ & 3-isobutyl-1-methylxanthine & -78.1 & 3.41 \\
\hline \multirow[t]{4}{*}{ LmajPTR1 } & $1 \mathrm{e} 7 \mathrm{w}$ & methotrexate & -147.1 & 5.63 \\
\hline & $1 w 0 c$ & 2,4,6-triaminoquinazoline & -72.7 & 0.52 \\
\hline & $2 \mathrm{bf} 7$ & 7,8-dihydrobiopterin & -93.7 & 0.61 \\
\hline & $3 \mathrm{~h} 4 \mathrm{v}$ & $\begin{array}{l}\text { methyl 1-(4- }\{[(2,4-\text { diaminopteridin-6- } \\
\text { yl)methyl }] \text { amino }\} \text { benzoyl }) \text { piperidine-4-carboxylate }\end{array}$ & -129.9 & 5.50 \\
\hline LmajMetRS & $3 \mathrm{kfl}$ & methionyl-adenylate & -172.5 & 3.35 \\
\hline \multirow[t]{2}{*}{ LmajTyrRS } & $3 \mathrm{p} 0 \mathrm{~h}$ & $3,7,3^{\prime}, 4^{\prime}$-tetrahydroxyflavone & -90.3 & 0.56 \\
\hline & $3 \mathrm{p} 0 \mathrm{j}$ & tyrosinol & -77.2 & 1.42 \\
\hline \multirow[t]{2}{*}{ LmajUGPase } & 2oef & none & --- & \\
\hline & $20 e g$ & uridine-5'-phosphate-glucose & -143.9 & 3.69 \\
\hline LdonCatB & homology & none & --- & \\
\hline \multirow[t]{2}{*}{ LdonCyp } & $2 \mathrm{haq}$ & none & --- & \\
\hline & 3 eov & omitted & --- & \\
\hline LdonDHODH & $3 \mathrm{c} 61$ & orotic acid & -64.2 & 9.23 \\
\hline LdonNMT & 2 wuu & none & --- & \\
\hline \multirow[t]{2}{*}{ LmexGAPDH } & $1 \mathrm{a} 7 \mathrm{k}$ & none & --- & \\
\hline & 1gyp & none & --- & \\
\hline
\end{tabular}


Table 41. Cont.

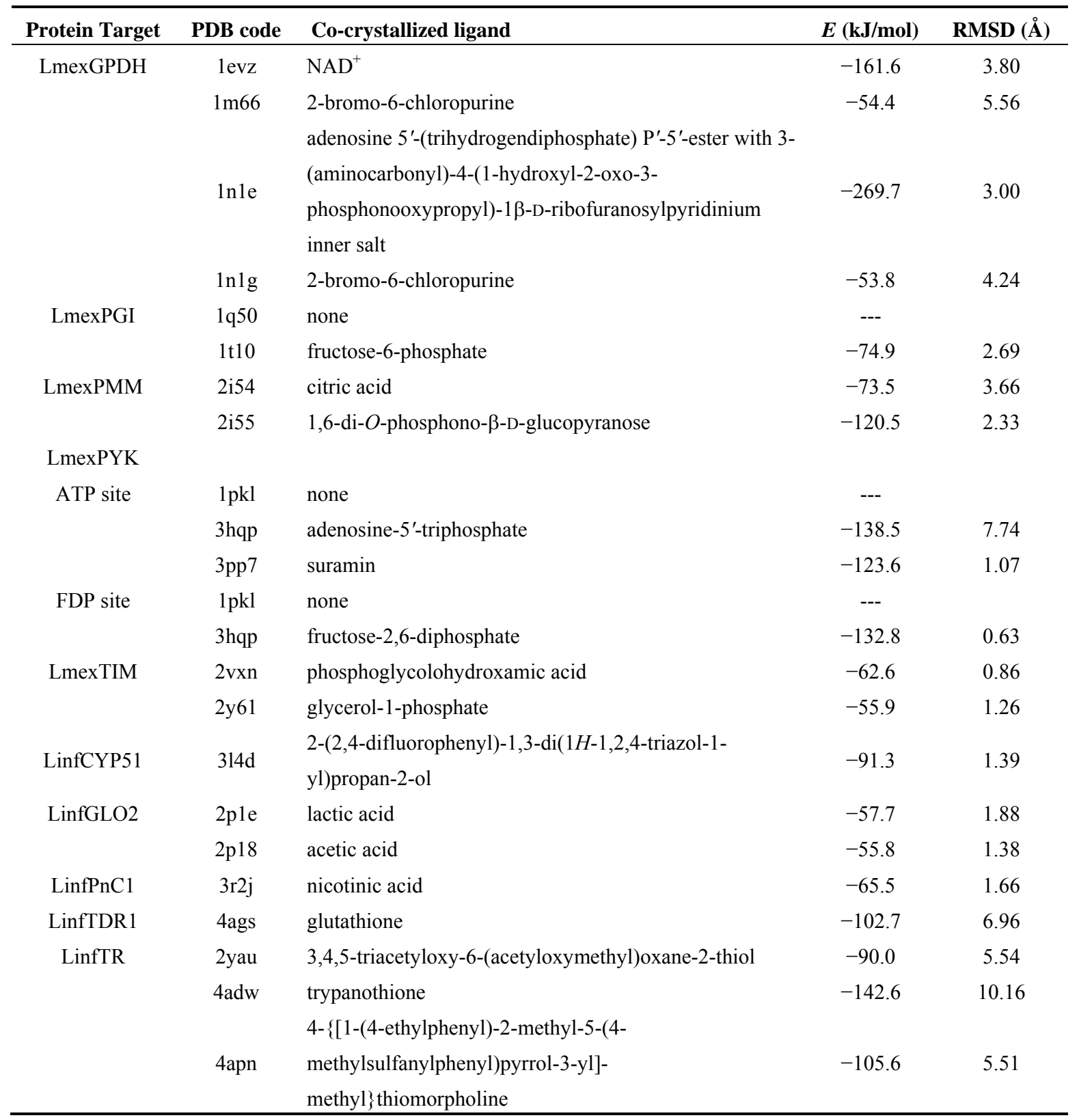

\section{Conclusions}

Numerous antiparasitic plant-derived natural products have been identified but the molecular target(s) of most of these compounds remain unknown. This gap in knowledge impedes further characterization and optimization of the antiparasitic activity of many of these compounds. In this molecular docking study, we have identified molecular targets in Leishmania that preferentially interact with certain classes of antiparasitic isoprenoids from plants. Consequently, Leishmania proteins that have structural motifs similar to those identified in this work may be explored as potential drug targets by antileishmanial drug discovery programs. It is important to point out that: (a) there are likely additional Leishmania proteins or other biochemical targets that have not yet been identified; (b) some of the antiparasitic terpenoids examined in the study may have poor bioavailability due to limited solubility, membrane permeability, hydrolysis, or other metabolic transformations; (c) the 
ligands may also target homologous isozymes in humans. Therefore, pharmacokinetic and pharmacodymanic studies as well as structure-based design and optimization studies are needed to resolve issues of bioavailability and selectivity. In summary, this in-silico molecular docking study has provided evidence for what classes and structural types of terpenoids may be targeting certain Leishmania protein targets and could provide the framework for synthetic modification of antiparasitic terpenoids, de novo synthesis of structural designs, and further phytochemical investigations.

\section{Acknowledgments}

We are grateful to Joseph $\mathrm{Ng}$ (Department of Biological Sciences, University of Alabama in Huntsville) for generating the structures of L. donovani cathepsin B and L. major cathepsin B.

\section{Conflict of Interest}

The authors declare no conflict of interest.

\section{References}

1. WHO Technical Report Series 949, 2010. Report of a Meeting of the WHO Expert Committee on the Control of Leishmaniases. Available online: http://apps.who.int/iris/bitstream/10665/44412/ 1/WHO_TRS_949_eng.pdf(accessed on 15 April 2013).

2. Barrett, M.P.; Mottram, J.C.; Goombs, G.H. Recent advances in identifying and validating drug targets in trypanosomes and leishmanias. Trends Microbiol. 1999, 7, 82-88.

3. Chawla, B.; Madhubala, R. Drug targets in Leishmania. J. Parasit. Dis. 2010, 34, 1-13.

4. Crowther, G.J.; Shanmugam, D.; Carmona, S.J.; Doyle, M.A.; Herz-Fowler, C.; Berriman, M.; Nwaka, S.; Ralph, S.A.; Roos, D.S.; Van Voorhis, W.C.; et al. Identification of attractive drug targets in neglected disease pathogens using an in silico approach. PLoS Negl. Trop. Dis. 2010, 4, e804.

5. Schmidt, T.J.; Khalid, S.A.; Romanha, A.J.; Alves, T.M.A.; Biavatti, M.W.; Brun, R.; Da Costa, F.B.; de Castro, S.L.; Ferreira, V.F.; de Lacerda, M.V.G.; et al. The potential of secondary metabolites from plants as drugs or leads against protozoan neglected ideases-Part I. Curr. Med. Chem. 2012, 19, 2128-2175.

6. Rigden, D.J.; Phillips, S.E.V.; Michels, P.A.M.; Fothergill-Gilmore, L.A. The structure of pyruvate kinase from Leishmania mexicana reveals details of the allosteric transition and unusual effector specificity. J. Mol. Biol. 1999, 291, 615-635.

7. Morgan, H.P.; Walsh, M.J.; Blackburn, E.A.; Wear, M.A.; Boxer, M.B.; Shen, M.; Veith, H.; McNae, I.W.; Nowicki, M.W.; Michels, P.A.; et al. A new family of covalent inhibitors block nucleotide binding to the active site of pyruvate kinase. Biochem. J. 2012, 448, 67-72.

8. Cordeiro, A.T.; Michels, P.A.; Delboni, L.F.; Thiemann, O.H. The crystal structure of glucose-6phosphate isomerase from Leishmania mexicana reveals novel active site features. Eur. J. Biochem. 2004, 271, 2765-2772.

9. Arsenieva, D.; Appavu, B.L.; Mazock, G.H.; Jeffery, C.J. Crystal structure of phosphoglucose isomerase from Trypanosoma brucei complexed with glucose-6-phosphate at $1.6 \AA$ resolution. Proteins 2008, 74, 72-80. 
10. Steiner, T.; Lamerz, A.C.; Hess, P.; Breithaupt, C.; Krapp, S.; Bourenkov, G.; Huber, R.; Gerardy-Schahn, R.; Jacob, U. Open and closed structures of the UDP-glucose pyrophosphorylase from Leishmania major. J. Biol. Chem. 2007, 282, 13003-13010.

11. Kim, H.; Hol, W.G. Crystal structure of Leishmania mexicana glycosomal glyceraldehyde-3phosphate dehydrogenase in a new crystal form confirms the putative physiological active site structure. J. Mol. Biol. 1998, 278, 5-11.

12. Guido, R.V.C.; Oliva, G.; Montanari, C.A.; Andricopulo, A.D. Structural basis for selective inhibition of trypanosomatid glyceraldehyde-3-phosphate dehydrogenase: Molecular docking and 3D QSAR studies. J. Chem. Inf. Model. 2008, 48, 918-929.

13. Zhang, W.W.; McCall, L.I.; Matlashewski, G. Role of cytosolic glyceraldehyde-3-phosphate dehydrogenase in visceral organ infection by Leishmania donovani. Eukaryot. Cell 2013, 12, 70-77.

14. Suresh, S.; Turley, S.; Opperdoes, F.R.; Michels, P.A.; Hol, W.G. A potential target enzyme for trypanocidal drugs revealed by the crystal structure of NAD-dependent glycerol-3-phosphate dehydrogenase from Leishmania mexicana. Structure 2000, 8, 541-552.

15. Choe, J.; Guerra, D.; Michels, P.A.; Hol, W.G. Leishmania mexicana glycerol-3-phosphate dehydrogenase showed conformational changes upon binding a bi-substrate adduct. J. Mol. Biol. 2003, 329, 335-349.

16. Olivares-Illana, V.; Pérez-Montfort, R.; López-Calahorra, F.; Costas, M.; Rodríguez-Romero, A.; Tuena de Gómez-Puyou, M.; Gómez-Puyou, A. Structural differences in triosephosphate isomerase from different species and discovery of a multitrypanosomatid inhibitor. Biochemistry 2006, 45, 2556-2560.

17. Alahuhta, M.; Wierenga, R.K. Atomic resolution crystallography of a complex of triosephosphate isomerase with a reaction-intermediate analog: New insight in the proton transfer reaction mechanism. Proteins 2010, 78, 1878-1888.

18. Kumar, K.; Bhargava, P.; Roy, U. Cloning, overexpression and characterization of Leishmania donovani triosephosphate isomerase. Exp. Parasitol. 2012, 130, 430-436.

19. Fyfe, P.K.; Westrop, G.D.; Silva, A.M.; Coombs, G.H.; Hunter, W.N. Leishmania TDR1 structure, a unique trimeric glutathione transferase capable of deglutathionylation and antimonial prodrug activation. Proc. Natl. Acad. Sci. USA 2012, 109, 11693-11698.

20. Garami, A.; Mehlert, A.; Ilg, T. Glycosylation defects and virulence phenotypes of Leishmania mexicana phosphomannomutase and dolicholphosphate-mannose synthase gene deletion mutants. Mol. Cell Biol. 2001, 21, 8168-8183.

21. Silva, M.S.; Barata, L.; Ferreira A.E.N.; Romão, S.; Tomás, A.M.; Freire, A.P.; Cordeiro, C. Catalysis and structural properties of Leishmania infantum glyoxalase II: Trypanothione specificity and phylogeny. Biochemistry 2008, 47, 195-204.

22. Padmanabhan, P.K.; Mukherjee, A.; Madhubala, R. Characterization of the gene encoding glyoxalase II from Leishmania donovani: A potential target for anti-parasite drugs. Biochem. J. 2006, 393, 227-234.

23. Silva, M.S.; Ferreira, A.E.N.; Tomás, A.M.; Cordeiro, C.; Freire, A.P. Quantitative assessment of the glyoxalase pathway in Leishmania infantum as a therapeutic target by modeling and computer simulation. FEBS J. 2005, 272, 2388-2398. 
24. Sajid, M.; McKerrow, J.H. Cysteine proteases of parasitic organisms. Mol. Biochem. Parasitol. 2002, 120, 1-21.

25. Paladi, C.D.S.; Pimentel, I.A.; Katz, S.; Cunha, R.L.; Judice, W.A.; Caires, A.C.; Barbiéri, C.L. In vitro and in vivo activity of a palladacycle complex on Leishmania (Leishmania) amazonensis. PLoS Negl. Trop. Dis. 2012, 6, e1626.

26. Coetzer, T.H.T.; Goldring, J.P.D.; Huson, L.E.J. Oligopeptidase B: A processing peptidase involved in pathogenesis. Biochemie 2008, 90, 336-344.

27. McLuskey, K.; Paterson, N.G.; Bland, N.D.; Isaacs, N.W.; Mottram, J.C. Crystal structure of Leishmania major oligopeptidase $\mathrm{B}$ gives insight into the enzymatic properties of a trypanosomatid virulence factor. J. Biol. Chem. 2010, 285, 39249-39259.

28. Swenerton, R.K.; Zhang, S.; Sajid, M.; Medzihradszky, K.F.; Craik, C.S.; Kelly, B.L.; McKerrow, J.H. The oligopeptidase B of Leishmania regulates parasite enolase and immune evasion. J. Biol. Chem. 2011, 286, 429-240.

29. Cordeiro, A.T.; Feliciano, P.R.; Pinkeiro, M.P.; Nonato, M.C. Crystal structure of dihydroorotate dehydrogenase from Leishmania major. Biochemie 2012, 94, 1739-1748.

30. Pinheiro, M.P.; Emery, F.D.S.; Nonato, M.C. Target sites for the design of anti-trypanosomatid drugs based on the structure of dihydroorotate dehydrogenase. Curr. Pharm. Des. 2013, 19, 2615-2627.

31. Camacho, A.; Hidalgo-Zarco, F.; Bernier-Villamor, V.; Ruiz-Pérez, L.M.; González-Pacanowska, D. Properties of Leishmania major dUTP nucleotidohydrolase, a distinct nucleotide-hydrolysing enzyme in kinetoplastids. Biochem. J. 2000, 346, 163-168.

32. Nguyen, C.; Kasinathan, G.; Leal-Cortijo, I.; Musso-Buendia, A.; Kaiser, M.; Brun, R.; Ruiz-Pérez, L.M.; Johansson, N.G.; González-Pacanowska, D.; Gilbert, I.H. Deoxyuridine triphosphate nucleotidohydrolase as a potential antiparasitic drug target. J. Med. Chem. 2005, 48, 5942-5954.

33. Hemsworth, G.R.; Moroz, O.V.; Fogg, M.J.; Scott, B.; Bosch-Navarrete, C.; González-Pacanowska, D.; Wilson, K.S. The crystal structure of the Leishmania major deoxyuridine triphosphate nucleotidohydrolase in complex with nucleotide analogues, dUMP, and deoxyuridine. J. Biol. Chem. 2011, 286, 16470-16481.

34. Gazanion, E.; Garcia, D.; Silvestre, R.; Gérard, C.; Guichou, J.F.; Labesse, G.; Seveno, M.; Cordeiro-Da-Silva, A.; Ouaissi, A.; Sereno, D.; et al. The Leishmania nicotinamidase is essential for $\mathrm{NAD}^{+}$production and parasite proliferation. Mol. Microbiol. 2011, 82, 21-38.

35. Shi, W.; Schramm, V.L.; Almo, S.C. Nucleoside hydrolase from Leishmania major. Cloning, expression, catalytic properties, transition state inhibitors, and the $2.5-\AA$ crystal structure. J. Biol. Chem. 1999, 274, 21114-21120.

36. Cui, L.; Rajasekariah, G.R.; Martin, S.K. A nonspecific nucleoside hydrolase from Leishmania donovani: Implications for purine salvage by the parasite. Gene 2001, 280, 153-162.

37. Rennó, M.N.; França, T.C.; Nico, D.; Palatnik-de-Sousa, C.B.; Tinoco, L.W.; Figueroa-Villar, J.D. Kinetics and docking studies of two potential new inhibitors of the nucleoside hydrolase from Leishmania donovani. Eur. J. Med. Chem. 2012, 56, 301-307.

38. Souza, T.A.C.B.; Trindade, D.M.; Tonoli, C.C.C.; Santos, C.R.; Ward, R.J.; Arni, R.K.; Oliveira, A.H.C.; Murakami, M.T. Molecular adaptability of nucleoside diphosphate kinase b from trypanosomatid parasites: Stability, oligomerization and structural determinants of nucleotide binding. Mol. BioSyst. 2011, 7, 2189-2195. 
39. Seebeck, T.; Gong, K.W.; Kunz, S.; Schaub, R.; Shalaby, T.; Zorghi, R. cAMP signaling in Trypanosoma brucei. Int. J. Parasitol. 2001, 31, 491-498.

40. Wang, H.; Yan, Z.; Geng, J.; Kunz, S.; Seebeck, T.; Ke, H. Crystal structure of the Leishmania major phosphodiesterase LmjPDEB1 and insight into the design of the parasite-selective inhibitors. Mol. Microbiol. 2007, 66, 1029-1038.

41. Malki-Feldman, L.; Jaffe, C.L. Leishmania major: Effect of protein kinase A and phosphodiesterase activity on infectivity and proliferation of promastigotes. Exp. Parasitol. 2009, 123, 39-44.

42. Nare, B.; Luba, J.; Hardy, L.W.; Beverly, S. New approaches to Leishmania chemotherapy: Pteridine reductase 1 (PTR1) as a target and modulator of antifolate sensitivity. Parasitology 1997, 114, S101-S110.

43. Gourley, D.G.; Schüttelkopf, A.W.; Leonard, G.A.; Luba, J.; Hardy, L.W.; Beverley, S.M.; Hunter, W.N. Pteridine reductase mechanism correlates pterin metabolism with drug resistance in trypanosomatid parasites. Nat. Struct. Biol. 2001, 8, 521-525.

44. Kaur, J.; Kumar, P.; Tyagi, S.; Pathak, R.; Batra, S.; Singh, P.; Singh, N. In silico screening, structure-activity relationship, and biologic evaluation of selective pteridine reductase inhibitors targeting visceral leishmaniasis. Antimicrob. Agents Chemother. 2011, 55, 659-666.

45. Guerrieri, D.; Ferrari, S.; Costi, M.P.; Michels, P.A. Biochemical effects of riluzole on Leishmania parasites. Exp. Parasitol. 2013, 133, 250-254.

46. Price, H.P.; Menon, M.R.; Panethymitaki, C.; Goulding, D.; McKean, P.G.; Smith, D.F. Myristoyl-CoA: Protein $N$-myristoyltransferase, an essential enzyme and potential drug target in kinetoplastid parasites. J. Biol. Chem. 2003, 278, 7206-7214.

47. Frearson, J.A.; Brand, S.; McElroy, S.P.; Cleghorn, L.A.; Smid, O.; Stojanovski, L.; Price, H.P.; Guther, M.L.; Torrie, L.S.; Robinson, D.A.; et al. $N$-myristoyltransferase inhibitors as new leads to treat sleeping sickness. Nature 2010, 464, 728-732.

48. Brannigan, J.A.; Smith, B.A.; Yu, Z.; Brzozowski, A.M.; Hodgkinson, M.R.; Maroof, A.; Price, H.P.; Meier, F.; Leatherbarrow, R.J.; Tate, E.W.; et al. N-myristoyltransferase from Leishmania donovani: Structural and functional characterisation of a potential drug target for visceral leishmaniasis. J. Mol. Biol. 2010, 396, 985-999.

49. Venugopal, V.; Sen, B.; Datta, A.K.; Banerjee, R. Structure of cyclophilin from Leishmania donovani at 1.97 Å resolution. Acta Crystallogr. Sect. F 2007, F63, 60-64.

50. Yau, W.L.; Blisnick, T.; Taly, J.F.; Helmer-Citterich, M.; Schiene-Fischer, C.; Leclercq, O.; Li, J.; Schmidt-Arras, D.; Morales, M.A.; Notredame, C.; et al. Cyclosporin A treatment of Leishmania donovani reveals stage-specific functions of cyclophilins in parasite proliferation and viability. PLoS Negl. Trop. Dis. 2010, 4, e729.

51. Larson, E.T.; Kim, J.E.; Zucker, F.H.; Kelley, A.; Mueller, N.; Napuli, A.J.; Verlinde, C.L.M.J.; Fan, E.; Buckner, F.S.; Van Voorhis, W.C.; et al. Structure of Leishmania major methionyl-tRNA synthetase in complex with intermediate products methionyladenylate and pyrophosphate. Biochemie 2011, 93, 570-582.

52. Larson, E.T.; Kim, J.E.; Castaneda, L.J.; Napuli, A.J.; Zhang, Z.; Fan, E.; Zucker, F.H.; Verlinde, C.L.; Buckner, F.S.; Van Voorhis, W.C.; et al. The double-length tyrosyl-tRNA synthetase from the eukaryote Leishmania major forms an intrinsically asymmetric pseudo-dimer. J. Mol. Biol. 2011, 3, 159-176. 
53. Chan-Bacab, M.J.; Peña-Rodríguez, LM. Plant natural products with leishmanicidal activity. Nat. Prod. Rep. 2001, 18, 674-688.

54. Rocha, L.G.; Almeida, J.R.G.S.; Macêdo, R.O.; Barbosa-Filho, J.M. A review of natural products with antileishmanial activity. Phytomedicine 2005, 12, 514-535.

55. Salem, M.M.; Werbovetz, K.A. Natural products from plants as drug candidates and lead compounds against leishmaniasis and trypanosomiasis. Curr. Med. Chem. 2006, 13, 2571-2598.

56. Polonio, T.; Efferth, T. Leishmaniasis: Drug resistance and natural products (Review). Int. J. Mol. Med. 2008, 22, 277-286.

57. Mishra, B.B.; Singh, R.K.; Strivastava, A.; Tripathi, V.J.; Tiwari, V.K. Fighting against leishmaniasis: Search of alkaloids as future true potential anti-leishmanial agents. Mini-Rev. Med. Chem. 2009, 9, 107-123.

58. Sen, R.; Chatterjee, M. Plant derived therapeutics for the treatment of leishmaniasis. Phytomedicine 2011, 18, 1056-1069.

59. Ogungbe, I.V.; Singh, M.; Setzer, W.N. Antileishmanial natural products from plants. Stud. Nat. Prod. Chem. 2012, 36, 331-382.

60. Schmidt, T.J.; Khalid, S.A.; Romanha, A.J.; Alves, T.M.A.; Biavatti, M.W.; Brun, R.; Da Costa, F.B.; de Castro, S.L.; Ferreira, V.F.; de Lacerda, M.V.G.; et al. The potential of secondary metabolites from plants as drugs or leads against protozoan neglected diseases-Part II. Curr. Med. Chem. 2012, 19, 2176-2228.

61. Monzote, L.; Alarcón, O.; Setzer, W.N. Antiprotozoal activity of essential oils. Agric. Consp. Sci. 2012, 77, 167-175.

62. Congreve, M.; Chessari, G.; Tisi, D.; Woodhead, A.J. Recent developments in fragment-based drug discovery. J. Med. Chem. 2008, 51, 3661-3680.

63. Zoete, V.; Grosdidier, A.; Michielin, O. Docking, virtual high throughput screening and in silico fragment-based drug design. J. Cell Mol. Med. 2009, 13, 238-248.

64. Lekphrom, R.; Kanokmedhakul, S.; Kanokmedhakul, K. Bioactive diterpenes from the aerial parts of Anisochilus harmandii. Planta Med. 2010, 76, 726-728.

65. Yuan, T.; Zhu, R.X.; Zhang, H.; Odeku, O.A.; Yang, S.P.; Liao, S.G.; Yue, J.M. Structure determination of grandifotane A from Khaya grandifoliola by NMR, X-ray diffraction, and ECD calculation. Org. Lett. 2010, 12, 252-255.

66. Idowu, O.A.; Soniran, O.T.; Ajana, O.; Aworinde, D.O. Ethnobotanical survey of antimalarial plants used in Ogun State, southwest Nigeria. Afr. J. Pharm. Pharmacol. 2010, 4, 55-60.

67. Bickii, J.; Njifutie, N.; Foyere, J.A.; Basco, L.K.; Ringwald, P. In vitro antimalarial activity of limonoids from Khaya grandifoliola C.D.C. (Meliaceae). J. Ethnopharmacol. 2000, 69, 27-33.

68. Sartorelli, P.; Andrade, S.P.; Melhem, M.S.C.; Prado, F.O.; Tempone, A.G. Isolation of antileishmanial sterol from the fruits of Cassia fistula using bioguided fractionation. Phytother. Res. 2007, 21, 644-647.

69. Hoet, S.; Pieters, L.; Muccioli, G.G.; Habib-Jiwan, J.L.; Opperdoes, F.R.; Quetin-Leclercq, J. Antitrypanosomal activity of triterpenoids and sterols from the leaves of Strychnos spinosa and related compounds. J. Nat. Prod. 2007, 70, 1360-1363.

70. Nweze, N.E.; Anene, B.M.; Asuzu, I.U. In vitro anti-trypanosomal activities of crude extracts, $\beta$-sitosterol and $\alpha$-sulfur from Buchholzia coriacea seed. Afr. J. Biotechnol. 2011, 10, 15626-15632. 
71. Setzer, W.N.; Ogungbe, I.V. In-silico investigation of antitrypanosomal phytochemicals from Nigerian medicinal plants. PLoS Negl. Trop. Dis. 2012, 6, e1727.

72. Kerr, I.D.; Wu, P.; Marion-Tsukamaki, R.; Mackey, Z.B.; Brinen, L.S. Crystal structures of $\mathrm{TbCatB}$ and rhodesain, potential chemotherapeutic targets and major cysteine proteases of Trypanosoma brucei. PLoS Negl. Trop. Dis. 2010, 4, e701.

73. Cordeiro, A.T.; Feliciano, P.R.; Nonato, M.C. Crystal structure of Leishmania major dihydroorotate dehydrogenase. Biochimie 2012, doi:10.2210/pdb3gye/pdb.

74. Cheleski, J.; Rocha, J.R.; Pinheiro, M.P.; Wiggers, H.J.; da Silva, A.B.F.; Nonato, M.C.; Montanari, C.A. Novel insights for dihydroorotate dehydrogenase class 1A inhibitors discovery. Eur. J. Med. Chem. 2010, 45, 5899-5909.

75. Brand, S.; Cleghorn, L.A.; McElroy, S.P.; Robinson, D.A.; Smith, V.C.; Hallyburton, I.; Harrison, J.R.; Norcross, N.R.; Spinks, D.; Bayliss, T.; et al. Discovery of a novel class of orally active trypanocidal $N$-myristoyltransferase inhibitors. J. Med. Chem. 2012, 55, 140-152.

76. McLuskey, K.; Gibellini, F.; Carvalho, P.; Avery, M.A.; Hunter, W.N. Inhibition of Leishmania major pteridine reductase by 2,4,6-triaminoquinazoline: Structure of the NADPH ternary complex. Acta Crystallogr. Sect. D 2004, D60, 1780-1785.

77. Schüttelkopf, A.W.; Hardy, L.W.; Beverley, S.M.; Hunter, W.N. Structures of Leishmania major pteridine reductase complexes reveal the active site features important for ligand binding and to guide inhibitor design. J. Mol. Biol. 2005, 352, 105-116.

78. Cavazzuti, A.; Paglietti, G.; Hunter, W.N.; Gamarro, F.; Piras, S.; Loriga, M.; Allecca, S.; Corona, P.; McLuskey, K.; Tulloch, L.; et al. Discovery of potent pteridine reductase inhibitors to guide antiparasite drug development. Proc. Natl. Acad. Sci. USA 2008, 105, 1448-1453.

79. Venugopal, V.; Datta, A.K.; Bhattacharyya, D.; Dasgupta, D.; Banerjee, R. Structure of cyclophilin from Leishmania donovani bound to cyclosporin at $2.6 \AA$ resolution: Correlation between structure and thermodynamic data. Acta Crystallogr. Sect. D 2009, D65, $1187-1195$.

80. Arakaki, T.L.; Merritt, E.A.; Ullman, B.; Yates, P.A. Crystal structure of dihydroorotate dehydrogenase from Leishmania donovani. 2013, To be published, doi:10.2210/pdb3c61/pdb.

81. Kim, H.; Feil, I.K.; Verlinde, C.L.; Petra, P.H.; Hol, W.G. Crystal structure of glycosomal glyceraldehyde-3-phosphate dehydrogenase from Leishmania mexicana: Implications for structure-based drug design and a new position for the inorganic phosphate binding site. Biochemistry 1995, 34, 14975-14986.

82. Choe, J.; Suresh, S.; Wisedchaisri, G.; Kennedy, K.J.; Gelb, M.H.; Hol, W.G. Anomalous differences of light elements in determining precise binding modes of ligands to glycerol-3phosphate dehydrogenase. Chem. Biol. 2002, 9, 1189-1197.

83. Kedzierski, L.; Malby, R.L.; Smith, B.J.; Perugini, M.A.; Hodder, A.N.; Ilg, T.; Colman, P.M.; Handman, E. Structure of Leishmania mexicana phosphomannomutase highlights similarities with human isoforms. J. Mol. Biol. 2006, 363, 215-227.

84. Morgan, H.P.; McNae, I.W.; Nowicki, M.W.; Hannaert, V.; Michels, P.A.; Fothergill-Gilmore, L.A.; Walkinshaw, M.D. Allosteric mechanism of pyruvate kinase from Leishmania mexicana uses a rock and lock model. J. Biol. Chem. 2010, 285, 12892-12898. 
85. Morgan, H.P.; McNae, I.W.; Nowicki, M.W.; Zhong, W.; Michels, P.A.; Auld, D.S.; Fothergill-Gilmore, L.A.; Walkinshaw, M.D. The trypanocidal drug suramin and other trypan blue mimetics are inhibitors of pyruvate kinases and bind to the adenosine site. J. Biol. Chem. 2011, 286, 31232-31240.

86. Venkatesan, R.; Alahuhta, M.; Pihko, P.M.; Wierenga, R.K. High resolution crystal structures of triosephosphate isomerase complexed with its suicide inhibitors: The conformational flexibility of the catalytic glutamate in its closed, liganded active site. Protein Sci. 2011, 20, 1387-1397.

87. Hargrove, T.Y.; Wawrzak, Z.; Liu, J.; Nes, W.D.; Waterman, M.R.; Lepesheva, G.I. Substrate preferences and catalytic parameters determined by structural characteristics of sterol $14 \alpha-$ demethylase (CYP51) from Leishmania infantum. J. Biol. Chem. 2011, 286, 26838-26848.

88. Ilari, A.; Baiocco, P.; Messori, L.; Fiorillo, A.; Boffi, A.; Gramiccia, M.; Di Muccio, T.; Coltti, G. A gold-containing drug against parasitic polyamine metabolism: The X-ray structure of trypanothione reductase from Leishmania infantum in complex with auranofin reveals a dual mechanism of enzyme inhibition. Amino Acids 2012, 42, 803-811.

89. Baiocco, P.; Ilari, A.; Colotti, G.; Malatesta, F.; Fiorillo, A. Crystal structure of Leishmania infantum trypanothione reductase in complex with NADPH and trypanothione. To be published, doi:10.2210/pdb4adw/pdb.

90. Baiocco, P.; Ilari, A.; Colotti, G.; Biava, M. Structure of TR from Leishmania infantum in complex with a diarylpirrole-based inhibitor. To be published, doi:10.2210/pdb4apn/pdb.

91. Molegro Virtual Docker v 5.0. Molegro ApS: Aarhus, Denmark, 2011.

92. Thomsen, R.; Christensen, M.H. MolDock: A new technique for high-accuracy molecular docking. J. Med. Chem. 2006, 49, 3315-3321.

93. Spartan '10 for Windows, v 1.1. : Wavefunction, Inc.: Irvine, CA, USA, 2011.

94. Halgren, T.A. Merck molecular force field. I. Basis, form, scope, parameterization, and performance of MMFF 94. J. Comput. Chem. 1996, 17, 490-519.

95. Collaborative Computational Project, Number 4. The CCP4 suite: Program for protein crystallography. The CCP4 suite: Programs for protein crystallography. Acta Crystallogr. D Biol. Crystallogr. 1994, 50, 760-763.

96. Stein, N. CHAINSAW: A program for mutating pdb files used as templates in molecular replacement. J. Appl. Crystallogr. 2008, 41, 641-643.

97. Brünger, A.T.; Adams, P.D.; Clore, G.M.; DeLano, W.L.; Gros, P.; Grosse-Kunstleve, R.W.; Jiang, J.S.; Kuszewski, J.; Nilges, M.; Pannu, N.S.; et al. Crystallography \& NMR System: A new software suite for macromolecular structure determination. Acta Crystallogr. D Biol. Crystallogr. 1998, 54, 905-921.

Sample Availability: Not available.

(C) 2013 by the authors; licensee MDPI, Basel, Switzerland. This article is an open access article distributed under the terms and conditions of the Creative Commons Attribution license (http://creativecommons.org/licenses/by/3.0/). 